Thomas Michael Baum

\title{
Staatsverschuldung und Stabilisierungspolitik in der Demokratie
}




\section{Staatsverschuldung und Stabilisierungspolitik in der Demokratie}

Die neoinstitutionalistische Kritik wendet sich gegen die durch den Keynesianismus eingeführte Möglichkeit der Defizitfinanzierung von Staatsausgaben, welche aufgrund politisch-ökonomischer Interdependenzen in westlichen Demokratien zu immer neuen und höheren Defiziten, einem wachsenden Staatsanteil und ständig steigenden Inflationsraten führt. Auf der Basis dieser Kritik wird eine Rückkehr zur Regel des jährlichen materiellen Haushaltsausgleichs gefordert. Die vorliegende Arbeit setzt sich kritisch mit der neoinstitutionalistischen Analyse und der Forderung eines jährlichen Budgetausgleichs auseinander. Der Autor relativiert diese Forderung durch allokative und stabilisatorische Argumente und schlägt Ergänzungsregelungen vor.

Thomas Baum wurde 1954 in Reutlingen geboren. Studium der Volkswirtschaftslehre von 1973 bis 1978 an den Universitäten Stuttgart und Tübingen. Seit November 1978 Wissenschaftlicher Mitarbeiter bei Prof. Dr. Cay Folkers am Lehrstuhl für Finanzwissenschaft der Universität Hohenheim. 
Staatsverschuldung und Stabilisierungspolitik in der Demokratie 


\section{Hohenheimer \\ Volkswirtschaftlicheschriften}

Herausgegeben von

Prof. Dr. Cay Folkers

Prof.Dr. Klaus Herdzina

Prof.Dr. Franz Mehler

Prof.Dr. Walter Piesch

Prof.Dr.Ingo Schmidt

Prof.Dr. Helmut Walter

Prof.Dr. Josua Werner

Band2

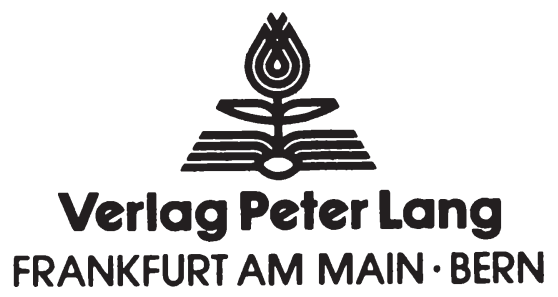




\section{Thomas Michael Baum}

\section{Staatsverschuldung und \\ Stabilisierungspolitik inder Demokratie \\ Zurneoinstitutionalistischen Kritik der keynesianischen Fiskalpolitik}

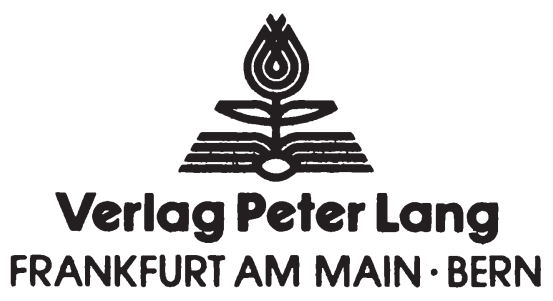


CIP-Kurztitelaufnahme der Deutschen Bibliothek

\section{Baum, Thomas Michael:}

Staatsverschuldung und Stabilisierungspolitik in der Demokratie : zur neoinstitutionalist. Kritik d. keynesian. Fiskalpolitik / Thomas Michael Baum. - Frankfurt am Main ; Bern : Lang, 1982.

(Hohenheimer volkswirtschaftliche Schriften ; Bd. 2) ISBN 3-8204-5782-8

Open Access: The online version of this publication is published on www.peterlang.com and www.econstor.eu under the international Creative Commons License CC-BY 4.0. Learn more on how you can use and share this work: http://creativecommons.org/licenses/by/4.0.

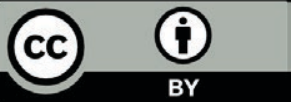

This book is available Open Access thanks to the kind support of ZBW - Leibniz-Informationszentrum Wirtschaft.

NE: GT

ISSN 0721-3085

ISBN 3-8204-5782-8

ISBN 978-3-631-75527-3 (eBook)

(C) Verlag Peter Lang GmbH, Frankfurt am Main 1982

Alle Rechte vorbehalten.

Nachdruck oder Vervielfältigung, auch auszugsweise, in allen Formen wie Mikrofilm, Xerographie, Mikrofiche, Mikrocard, Offset verboten.

Druck und Bindung: fotokop wilhelm weihert KG, darmstadt 
Seitdem keynesianische Ideen Eingang in die offizielle Wirtschaftspolitik gefunden haben, sind in allen westlichen staaten ständig wachsende Budgetdefizite, verstärkt steigende Staatsquoten und erhöhte Inflationstendenzen zu beobachten. Diese Entwicklung, die zu einem schwerwiegenden Problem für Wirtschaft und Staat geworden ist. steht im Mittelpunkt eines neuen Ansatzes der Kritik an herrschenden Grundsätzen finanzwirtschaftlicher Stabilisierungspolitik. Dabei werden erstmals die Effekte politischer und ökonomischer Institutionen auf das Ergebnis der stabilisierungspolitischen Entscheidungen des States systematisch untersucht.

Die vorliegende Studie unterzieht diese Zusammenhänge einer grundlegenden Analyse. Einerseits werden neue Erkenntnisse im Hinblick auf die theoretische Durchdringung der Beziehungen zwischen Keynesianik und politischen Entscheidungsprozessen vorgelegt, andererseits werden die bisher auf den angloamerikanischen Bereich beschränkten empirischen Analysen um Untersuchungen der z.T. erheblich divergierenden Verhältnisse in der Bundesrepublik Deutschland erweitert. Beide Aspekte lassen die Schrift als interessanten Beitrag zur aktuellen finanzpolitischen' Diskussion erscheinen.

Die Arbeit entstand während der Tätigkeit des Verfassers als Wissenschaftlicher Mitarbeiter im Rahmen des Forschungsprogramms des Lehrstuhls für Finanzwissenschaft der Universität Hohenheim. Sie eröffnet, nicht zuletzt im Hinblick auf die entworfenen verfassungsmäßigen Konsequenzen für die Staatsverschuldung, Ansätze zu weiteren Untersuchungen und $z u$ politischen Grundsatzdiskussionen. Es ist zu wüschen, daB die vorliegende Schrift sich als fruchtbarer Beitrag zur Lösung einer zentralen Frage der öffentlichen Finanzwirtschaft erweisen wird.

Stuttgart-Hohenheim, den 10.6.1982

Cay Folkers 
Thomas Michael Baum - 978-3-631-75527-3

Downloaded from PubFactory at 01/11/2019 03:51:25AM

via free access 
STATT EINER WIDMUNG

Mein besonderer Dank gilt meinem Doktorvater und Betreuer, Herrn Prof. Dr. Cay Folkers. Von seiner alzen Problemen aufgeschlossenen Haltung habe ich während der mehr als dreijährigen intensiven wissenschaftlichen Zusammenarbeit nicht nur bei der Anfertigung der vorliegenden Arbeit, sondern auch bei meiner Forschungsund Lehrtätigkeit als Assistent entscheidend profitiert.

Meiner Braut Martina danke ich für ihre Geduld in all den stunden, in denen sie einen nur für ökonomische Probleme aufgeschlossenen Partner ertragen mußte.

Für die finanzielle Unterstützung in Form eines Druckkostenzuschusses schulde ich der Landeszentralbank in Baden-Württemberg Dank.

Hohenheim, im Mai 1982

Thomas Baum 
Thomas Michael Baum - 978-3-631-75527-3

Downloaded from PubFactory at 01/11/2019 03:51:25AM

via free access 
INHALTSVERZE ICHNIS

Seite

I. EINLEITUNG

II. DIE STRUKTUR DER ARGUMENTATION VON BUCHANAN/WAGNER

III. DEMOKRATIE UND DEFIZITE

1. Theoretische Analyse der Kosten und Nutzen von Budgetüberschüssen und Budgetdefiziten unter alternativen Bedingungen

1.1. Kosten und Nutzen marginaler Budgetüberschüsse 14

1.2. Kosten und Nutzen marginaler Budgetdefizite 16

1.3. Die Auswirkungen alternativer politischer Voraussetzungen auf den Budgetsaldo

1.3.1. Die Möglichkeit einer "symmetrischen" Politik be $i$ Vorliegen der "Presuppositions of Harvey Road"

1.3.2. Der Budgetsaldo in einer Demokratie 18

1.4. Kritik der theoretischen Analyse 20

1.4.1. Vorgehensweise 20

1.4.2. Ein einfaches Modell 20

1.4.3. Der Budgetsaldo unter neoinstitutionalistischen Annahmen

1.4.4. Einige Bemerkungen zum Informationsproblem 28

1.4.5. Das Phänomen der Fiskalillusion 31

1.4.5.1. Zum Begriff der Fiskalillusion 31

1.4.5.2. Der EinfluB alternativer Einnahmeinstitutionen auf die Fiskalillusion 33

1.4.5.3. Der EinfluB politischer Abstimmungsregeln auf die Fiskaliliusion

1.4.5.4. Zur Kritik BRUNNERs an der Annahme der Fiskalillusion

1.4.5.5. Die Auswirkungen der Fiskalillusion auf die Entscheidung der Wähler für Defizite versus Oberschüsse

1.4.6. Alternative Wirkungen von Budgetsalden auf Realeinkommen und Inflationsrate 
Se ite

1.4.7. Unterschiedliche Zeitpräferenzen 43

1.4.8. Crowding-out-Effekte der Statsverschuldung 46

1.4.9. Verteilungswirkungen 47

1.4.10. Psychologische Grenzen der Staatsverschuldung 51

1.4.11. Fazit der theoretischen Argumente 52

2. Versuch einer Oberprüfung der BUCHANAN/WAGNER -
These anhand politometrischer Schätzungen

3. Fazit: Die Auswirkungen von Budgetdefiziten auf das Abstimmungsverhalten der Wähler

IV. DIE AUSWIRKUNGEN EINES SINKENDEN STEUERPREISES AUF DAS WACHSTUM DES STAATSANTEILS

1. Theoretische Grundlagen der BUCHANAN/WAGNER-These 66

2. Dynamische Analyse 71

2.1. Das Mode11 71

2.2. Folgerungen aus dem dynamischen Modell 79

2.3. Berücksichtigung des Statsausgabenmultiplikators und einkommensabhängiger Steuern 83

3. Empirische Relevanz der Ergebnisse 90

4. Folgerungen aus der Modellerweiterung 92

5. Defizite und Staatsanteil unter alternativen
Annahmen

6. Fazit: Die empirische Relevanz der BUCHANAN/WAGNERThese

V. DIE WIRKUNGEN VON BUDGETDEFIZITEN AUF GELDMENGE UND PRE ISN I VEAU

1. Die neoinstitutionalistische Argumentation 96

2. Kritik der neoinstitutionalistischen Argumentation 98

2.1. Das Argument eines zinsbedingten crowding-out

2.2. Die Reaktion der Zentralbank auf ein steigendes Zinsniveau. Eine Analyse der Geldmengen- und Inflationswirkungen staatlicher Budgetdefizite

2.2.1. Institutionelle Analyse der Zentralbankautonomie in der Bundesrepublik Deutschland und in den USA 
Seite

2.2.1.1. Die personelle Unabhängigkeit der Deutschen Bundesbank

106

2.2.1.2. Koordinierungsverflechtung und Unabhängigkeit der Deutschen Bundesbank

109

2.2.1.3. Die Unabhängigkeit des Federal Reserve System (FRS)

111

2.2.1.3.1. Die Struktur des amerikanischen Zentralbankensystems

2.2.1.3.2. Konsequenzen für die Zentralbankautonomie 114

2.2.1.3.2.1. Personelle Unabhängigkeit

2.2.1.3.2.2. Institutionelle Unabhängigkeit

115

2.2.1.4. Schlußfolgerungen aus der institutionellen Analyse der Zentralbankabhängigkeit

2.2.2. Empirische Analyse

2.2.2.1. Empirische Analysen der Zentralbankautonomie in der Bundesrepublik Deutschland

2.2.2.1.1. BASLERs Optimierungsmodell zur Bestimmung von Zielprioritäten der Deutschen Bundesbank

2.2.2.1.2. Der Satisfizierungsansatz von FREY und SCHNE IDER

2.2.2.1.3. Ein Satisfizierungsmodell zur Beurteilung der Bundesbankautonomie

2.2.2.1.4. Fazit: Die Autonomie der Deutschen Bundesbank

2.2.2.2. Empirische Analyse der Geldmengen- und Inflationswirkungen staatlicher Budgetdefi$z i t e$ in den USA und in der Bundesrepublik Deutschland

2.2.2.2.1. Vorgehenswe ise

2.2.2.2.2. Budgetdefizite, Geldmengenänderungen und Inflationsrate in den USA

2.2.2.2.2.1. Die historische Entwicklung der Budgetsalden und der Geldmenge in den USA einige Bemerkungen zur empirischen AnaIyse von BUCHANAN/WAGNER

2.2.2.2.2.2. Okonometrische Untersuchungen des $\mathrm{Zu}$ sammenhangs von Budgetdefiziten, Geldmenge und Inflation in den USA 
Se ite

2.2.2.2.2.3. Zusammenfassung der Ergebnisse für den Bereich der USA

2.2.2.2.3. Geldmengen- und Inflationswirkungen von Budgetdefiziten in der Bundesrepublik Deutschland

2.3. Fazit: Budgetdefizite, Geldmenge und Preisniveau in den USA und in der Bundesrepublik Deutschland

VI. ZUSAMMENFASSENDE WORDIGUNG DER NEOINSTITUTIONALISTI SCHEN KRITIK

VII. ZUR THERAPIE: BEURTEILUNG EINER ROCKKEHR ZUR REGEL

DES BUDGETAUSGLEICHS

1. Die Budgetausgleichsregel aus neoinstitutionalistischer Sicht

2. Der spezifische Vorschlag von BUCHANAN/WAGNER

3. Beurteilung des Vorschlags von BUCHANAN/WAGNER

3.1. Realisierungschancen und De-facto-Bindung der Politiker

3.2. Allokative Effizienz

3.3. Stabilisatorische Effizienz

183

3.4. Zusammenfassung

188

4. Ein alternativer Vorschlag zur Anderung der Finanzverfassung

VIII. DER NEOINSTITUTIONALISTISCHE BEITRAG ZUR FINANZPOLITIK

ANHANG

1. Zitate aus Interviews zur Unabhängigkeit des Federal Reserve System

2. Datenreihen zu Abschnitt V.2.2.2.1.3.

3. Eine "Reaktionsfunktion" der Zentralbank 
VERZEICHNIS DER ABBILDUNGEN

Se ite

Abbildung 1: Die Struktur der neoinstitutionalistischen Argumentationskette

Abbildung 2: Der Budgetsaldo in einer Demokratie 18

Abbildung 3: Wirkungen der Staatsverschuldung auf das Nutzenniveau eines Individuums mit Gegenwartspräferenz

Abbildung 4: Wirkungen der Statsverschuldung auf ein unterproportional besteuertes Individuum mit Gegenwartspräferenz

Abbildung 5: Fiskalpolitik und individuelle Präferenzen

Abbildung 6: Dynamische Entwicklung der Nachfrage nach öffentlichen Gütern

Abbildung 7: Die organisatorische Struktur des Federal Reserve System

Abbildung 8: Die Interdependenzen im Konfliktmodell von Frey und Schneider 
VERZEICHNIS DER TABELLEN

Se ite

Tabelle 1: Der Einfluß ökonomischer Variablen auf die Regierungspopularität

Tabelle 2: Dynamische Entwicklung bei Beibehaltung des stabilitätspolitisch bedingten Defizits

Tabelle 3: Dynamische Entwicklung bei Abbau des stabilitätspolitisch bedingten Defizits in Periode 2

Tabelle 4: Obersicht über die Veränderung der Staatsquote

Tabelle 5: Die dynamische Entwicklung bei steigenden Grenzkosten

Tabelle 6: Entwicklung des Bruttosozialprodukts und der Versorgung mit öffentlichen Gütern für $f=1$

Tabelle 7: (Teil a) Entwicklung des Bruttosozialprodukts und der Versorgung mit öffentlichen Gütern für $f=50$ und Aufrechterhaltung des stabilitätspolitisch bedingten Defizits

Tabelle 7: (Teil b) Entwicklung des Bruttosozialprodukts und der Versorgung mit öffentlichen Güitern für $f=50$ und Abbau des stabilitätspolitisch bedingten Defizits in Periode 2

Tabelle 8: Auswirkungen einer sehr hohen Steuerpreiselastizität

Tabelle 9: Zielpräferenzen der Deutschen Bundesbank 1958 bis 1974

Tabelle 10: Diskontpolitik der Deutschen Bundesbank. Test der Abhängigkeitsthese. OLS-Schätzungen mit Quartalsdaten für die Perioden 1969 III 1980 II und 1973 I - 1980 II

Tabelle 11: Diskontpolitik der Deutschen Bundesbank. Test auf Verfolgung des jeweils am meisten gefährdeten Ziels. OLS-Schätzung mit Quartalsdaten von 1973 I - 1980 II 
Se ite

Tabelle 12: Budgetdefizite der Zentralregierung (DEF), Anderung des Bestands an Staatspapieren im Portefeuille der Zentralbank ( $\triangle B I L L S F E D)$, Geldmenge $\left(M_{1}\right)$, reales Sozialprodukt ( $\left.Y r\right)$, jeweils in Mrd. \$, Preisindex des Bruttosozialprodukts (P) und Arbeitslosenquote ( $A L Q)$ in den USA. Zeitreihen von 1947 1974

Tabelle 13: Geldmengenwirkungen von Budgetsalden in den USA im Zeitraum von 1949 - 1974

Tabelle 14: Geldmengenwirkungen von Budgetdefiziten in den USA von 1949 - 1974

Tabelle 15: Geldmengenwirkungen von Budgetdefiziten in der Bundesrepublik Deutschland

Tabelle 16: Die Reaktion der Geldmengenänderungen auf die wirtschaftliche Entwicklung und auf wirtschaftspolitische Zielgrößen 1973 I 1980 I 
Thomas Michael Baum - 978-3-631-75527-3

Downloaded from PubFactory at 01/11/2019 03:51:25AM

via free access 


\section{EINLE ITUNG}

HARRY G. JOHNSON hat die Geschichte der Wirtschaftswissenschaften als eine Abfolge von Revolutionen charakterisiert ${ }^{1}$ ) und die monetaristische Revolution als erste bedeutsame Konterrevolution in der Entwicklung der Nationalökonomie bezeichnet ${ }^{2)}$. Um erfolgreich zu sein, muBte diese Konterrevolution - wie auch zuvor die keynesianische Theorie bei ihrer Attacke auf die Klassik - zunächst ein zentrales Problem der Gegenwart identifizieren, das die zur Orthodoxie gewordene Lehre nicht zu lösen in der Lage war. Sodann war eine neue Theorie zu entwickeln, die dieses Problem unter Einbeziehung der in der orthodoxen Theorie nicht widerlegbaren Elemente - besser bewältigen konnte. Schlieblich muBte diese neue Theorie einen gewissen Schwierigkeitsgrad aufweisen, der jungen, aufstrebenden Wissenschaftlern die Gelegenheit gab, sich gegenüber älteren zu profilieren, sie mußte eine neue, attraktivere Methodologie entwickeln und sie hatte der okonometrie ein neues Forschungsfeld zu vermittel ${ }^{3}$ ).

Die Monetaristen fanden das zentrale Problem in der zunehmenden Inflation, deren Bekämpfung sich die Keynesianer machtlos gegenübersahen. Die neue Theorie, die zu liefern war, formulierte MILTON FRIEDMAN in Gestalt seiner Neoquantitätstheorie ${ }^{4)}$. Diese verallgemeinerte im wesentlichen die KEYNESsche Liquiditätspräferenztheorie auf der Grundlage einer verfeinerten Analyse des Vermögens und seiner Beziehung zum Einkommen ${ }^{5)}$. Dennoch wies sie den erforderlichen Schwierigkeitsgrad auf, ehrgeizige junge Wissenschaftler herauszufordern. Als weiteres Novum wandte FRIEDMAN

1) Vgl. Johnson (1971), S. $196 \mathrm{f}$.

2) Vgl. ebenda, S. 198

3) Zur genaueren Kennzeichnung dieser Bedingungen für eine wissenschaftliche Revolution vgl. ebenda, S. $198 \mathrm{ff}$.

4) Vgl. Friedman (1956)

5) Vgl. auch Johnson (1971), S. 208 
eine Methodologie der positiven okonomie an, die nicht darauf abzielte, ein großes und bei empirischen Tests sehr aufwendiges Gleichungssystem zu finden, sondern wenige, aber wichtige Beziehungen zu untersuchen, aus denen dann auf das interessierende Ergebnis geschlossen werden kann ${ }^{1) 2}$ ). SchlieBlich präsentierte er den okonometrikern mit seiner Geldnachfragefunktion eine neue empirische Beziehung, deren Stabilität es zu testen galt ${ }^{3)}$.

Im Verlauf der keynesianisch-monetaristischen Kontroverse konnten einige zunächst diametral entgegengesetzte Positionen geklärt und einander angenähert werden. Die auf beiden Seiten üblichen Simplifikationen ${ }^{4}$, bei denen den Monetaristen von den Keynesianern vorgeworfen wurde, für sie spiele nur Geld eine Rolle, und den Keynesianern, für sie spiele Geld überhaupt keine Rolle, sind schlieBlich fruchtbaren Diskussionen gewichen, bei denen es um die relative Stärke und Verläßlichkeit geld- und fiskalpolitischer Impulse ${ }^{5)}$ geht ${ }^{6)}$. Hinzu kommt, daB die Neuinterpretation des KEYNESSchen Gedankenguts durch LEIJONHUFVUD ${ }^{7)}$ zu einer Rehabilitierung von KEYNES geführt hat.

Inzwischen sieht sich der Keynesianismus einer zweiten Konterrevolution gegenüber, der Kritik der Neoinstitutionalisten.

JAMES M. BUCHANAN und RICHARD E. WAGNER haben mit ihrer Schrift "Democracy in Deficit" 8 ) einen ähnlich provozierenden Angriff auf

1) Vgl. ebenda, S. 209 und Mayer (1975), S. 207

2) Zur Rechtfertigung dieser Methodologie vgl. auch Brunner (1976), S. $48 \mathrm{ff}$.

3) Vgl. Johnson (1971), S. 209

4) Vgl. hierzu Kalmbach (1973), S. 11

5) Zur Abgrenzung monetärer und fiskalischer Impulse vgl. Neumann (1973), S. $16 \mathrm{f}$.

6) Vgl. Johnson (1975a), S. 54

7) Vgl. Leijonhufvud (1967) und (1968)

8) Vgl. Buchanan/Wagner (1977) 
den Keynesianismus vorgetragen wie 1956 FRIEDMAN. Gleich drei zentrale Probleme, die nach ihrer Meinung miteinander zusammenhängen und sich gegenseitig verstärken, haben die neuen Konterrevolutionäre ${ }^{1)}$ herausgegriffen. Sie behaupten, die oberzeugung der okonomen und Politiker vom Keynesianismus und die Anwendung keynesianischer Rezepte in der praktischen Wirtschaftspolitik habe zwangsläufig zu

- wachsenden Budgetdefiziten,

- einem kontinuierlichen relativen Wachstum des öffentlichen Sektors zu Lasten des privaten Sektors und

- zu ständig steigenden Inflationsraten

geführt ${ }^{2)}$. Der Grund 1 iege darin, daB weder die ökonomischen, noch die politischen Voraussetzungen, von denen die Keynesianer bei ihren wirtschaftspolitischen Empfehlungen ausgehen, mit der Realität übereinstimmen ${ }^{3)}$. Gleichzeitig wenden sich BUCHANAN und WAGNER gegen die keynesianische Behauptung, das marktwirtschaftiche Sy-

1) Die Bezeichnung Neo-Institutionalismus wurde von Gäfgen (1977) zur Charakterisierung einer Gruppe von Okonomen verwendet, deren Hauptgegner nicht die Keynesianer, sondern die Neoklassiker sind. Er zählt hierzu John K. Galbraith, G. Myrdal, F. Perroux, u.a. Gäfgen kennzeichnet diese Gruppe dadurch, "daB sie Okonomie bewußt als Sozialwissenschaft verstehen und überzeugt sind, daB Probleme der modernen Wirtschaftsgesellschaften nur gelöst werden können, indem man soziale Systeme als sich entwickelnde Ganzheiten betrachtet, und da $B$ man die Wirtschaft als soziales Subsystem anzusehen habe, dessen Zusammenspiel mit anderen Teilsystemen und dem Ganzen der Gesellschaft in das Verständnis einbezogen werden müsse . . Weniger Zustände als soziale Prozesse - Sollen im Vordergrund stehen". Vgl. Gäfgen (1977), S. 151. Frey (1977), $\dot{S} .7 \dot{6} \mathrm{ff}$. reiht die von Gäfgen genannten okonomen zusammen mit Buchanan und einigen anderen in die Reihe der "Unorthodoxen" ein, welche ebenfalls die von Gäfgen genannten Merkmale aufweisen. Da Buchanan und Wagner diese Eigenschaften in verstärkter Form aufweisen und in ihren Beiträgen ausdrücklich von der zentralen Hypothese ausgehen, daß "Institutions matter" (vgl. Buchanan/Wagner (1977), S. 144), erscheint auch für sie die Bezeichnung Neoinstitutionalisten angebracht, obwohl das Gedankengut Buchanans eher eine Weiterentwicklung der Gedanken von Th. Hobbes (1651) als einen Rückgriff auf die Schriften der Institutionalisten T.B, Veblen, J.R. Commens, W.C. Mitchell, u.a. darstellt.

2) Vgl. Z.B. auch Buchanan/Wagner (1978c), S. 80

3) Vgl. hierzu neben Buchanan/Wagner (1977), S. $77 \mathrm{ff} .,(1978 \mathrm{c})$, S. $82 \mathrm{ff}$. und (1978d), S. $14 \mathrm{ff}$. speziell für den britischen Bereich Burton (1978), S. $47 \mathrm{ff}$. 
stem sei instabil und die Regierung habe sowohl die Fähigkeit als auch die Verpflichtung, diese Instabilität zu beseitigen ${ }^{1}$. Als neue Theorie präsentieren sie die "Public Choice Theory"2), eine Theorie, die eine Anwendung der ökonomischen Denkweise auf den politischen Bereich darstellt, explizit auf das Zusammenspiel von politischem und ökonomischem Sektor abstellt und darauf abhebt, daB und wie institutionelle Regelungen wie beispielsweise Steuergesetze oder Abstimmungsregeln das Ergebnis dieses Zusammenspiels beeinflussen können ${ }^{3}$ ).

Methodisch bedient sich BUCHANAN des sogenannten "Contractarian Paradigm"4), d.h. er geht vom Individuum aus, das sich in einer Gemeinschaft wie dem Staat mit anderen Individuen einigen muB, um eigene und gemeinsame Ziele zu erreichen ${ }^{5)}$. Charakteristisch für diesen Ansatz ist, daB er auf den ProzeB abhebt, durch den sich Individuen auf ein Ergebnis einigen, nicht auf das Ergebnis als solches ${ }^{6)}$. Entscheidend für das Ergebnis eines sozialen Interaktionsprozesses sind die Spielrege $n$, denen sich die Beteiligten unterwerfen müssen. Andern sich die Regeln, dann ändert sich das Ergebnis.

Dieser kontraktorientierten Betrachtungsweise entspringt auch die Therapie, die BUCHANAN und WAGNER vorschlagen, um die von ihnen genannten Probleme zu beheben. Sie verlangen eine Rückkehr von der keynesianischen Regel, nach der Defizite möglich sind, zur klassischen Regel des Budgetausgleichs. Abweichungen hiervon sollen nur möglich sein, wenn Senat und Repräsentantenhaus mit Zwei-Drittel-

1) Vgl. Z.B. Buchanan/Wagner (1978d), S. 14

2) Für den Oberblick vgl. Mueller (1976) und (1979).

3) Die Public-Choice-Theorie wird im Deutschen auch als Theorie der nichtmarktlichen Entscheidungen bezeichnet.

4) Vgl. hierzu Buchanan (1975a)

5) Dieser Ansatz ist allen Arbeiten Buchanans gemein. Er wird in Buchanan (1975) exakt ausgearbeitet und begründet.

6) Vgl. z.B. Buchanan (1975a), S. 228 
Mehrheit eine Situation nationalen Notstands attestieren ${ }^{1)}$. Die Regel des Budgetausgleichs soll das Entstehen der durch die Defizite verursachten Probleme verhindern.

Auch die anderen Bedingungen, die JOHNSON für das Gelingen einer Revolution aufgestellt hat, werden vom Public-Choice-Ansatz erfiullt. Die Erkenntnis, daß das politische und das ökonomische system zusammenhängen, und daB der politische Bereich mit Hilfe des ökonomischen Instrumentariums untersucht werden kann, hat zur Formulierung einer Vielzahl politisch-ökonomischer Modelle ${ }^{2}$ geführt, die schwierig genug sind, um für junge, ehrgeizige okonomen attraktiv zu sein. Weiterhin eröffnete sich für okonometriker, die politisch-ökonomische Gesamtmodelle oder wichtige Funktionen aus solchen Modellen ${ }^{3)}$ testen können, ein weites Betätigungsfeld ${ }^{4}$.

Ziel dieser Arbeit ist es, den neoinstitutionalistischen Angriff auf die Keynesianer kritisch zu analysieren und Konsequenzen für die Fiskalpolitik abzuleiten. Den Hauptgegenstand der Arbeit bildet die Diskussion der bereits genannten neoinstitutionalistischen Kernthesen. Zunächst wird die Auswirkung der Staatsverschuldung auf das Wachstum der Statsausgaben und des Statsanteils am Sozialprodukt in demokratischen Staaten untersucht. Sodann wird die These der inflationären Wirkung staatlicher Budgetdefizite diskutiert. Dabei wird auf die spezifischen Verhältnisse in den USA und in der Bundesrepublik Deutschland abgestellt. Voraussetzung für beide Untersuchungen bildet eine detaillierte mikroökonomische Analyse der Auswirkungen staatiicher Budgetsalden auf Wählerentscheidungen. Im Anschluß wird der Vorschlag von

1) Vgl. Buchanan/Wagner (1977), Kapitel 12, S. $173 \mathrm{ff}$.

2) Vgl. Z.B. Nordhaus (1975), MacRae (1977). Weitere Modelle diskutiert Dinkel (1977), S. $149 \mathrm{ff}$. Zum Aufbau und zur Struktur dieser Modelle vgl. Frey (1977), S. $165 \mathrm{ff}$, , (1978), S. $208 \mathrm{ff}$. und (1979a), S. 5 f. sowie Kirchgäßner (1979), S. 429 ff.

3) Zu denken ist Z.B. an sogenannte Popularitätsfunktionen. Vgl. Frey (1977), S. $183 \mathrm{ff}$.

4) Für diesen Bereich der Okonometrie hat sich die Bezeichnung Politometrie eingebürgert. Vgl. ebenda, S. 183 
BUCHANAN/WAGNER, welcher eine Rückkehr zur Regel des Budgetausgleichs vorsieht, im Hinblick auf seine allokativen und stabilisatorischen Konsequenzen diskutiert. Die Analyse schließt mit einem Vorschlag zur Modifizierung der von den Autoren vorgeschlagenen Verfassungsänderung. Im letzten Kapitel werden sodann die einzelnen Ergebnisse in einer abschließenden Würdigung zusammengefaBt. 
II. DIE STRUKTUR DER ARGUMENTATION VON BUCHANAN/WAGNER

BUCHANAN/WAGNER (im folgenden BW) gehen bei ihrer Kritik von der vorkeynesianischen Zeit aus, in der das Budget des Staates aus mikroökonomischer Sicht in Analogie zum Budget eines Haushalts untersucht wurde, und in der demzufolge Budgetdefizite nur in Notsituationen zugelassen waren ${ }^{1)}$. So stellen die beiden Autoren auch fest, daB Defizite in dieser Zeit vor allem in Kriegszeiten auftraten ${ }^{2)}$. In den Nachkriegsjahren wurden dann Oberschüsse erwirtschaftet und zum Abbau der Kriegsschulden verwendet ${ }^{3)}$. Allmählich gelang es jedoch den Keynesianern, zunächst die okonomen und dann die Politiker davon zu überzeugen, daß das marktwirtschaftliche system inhärent instabil sei, und daß den wirtschaftspolitikern die Aufgabe zufalle, das system zu stabilisieren ${ }^{4) 5}$. Die Tatsache, daß Budgetdefizite nun nicht mehr auf Situationen nationalen Notstands beschränkt waren, setzte in der Folgezeit eine Entwicklung in Gang, die zu immer gröBeren Budgetdefiziten, einem wachsenden Regierungssektor und $z u$ hohen Inflationsraten führte.

Die Argumentationskette verläuft wie folgt ${ }^{6}$ ):

Ausgangspunkt ist die Feststellung, da B Budgetentscheidungen in einer Demokratie nicht von einer unabhängigen Elite von Wissen-

1) Vgl. Buchanan/Wagner (1977), S. 10. Als Beispiel für die damalige Sicht zitieren die Autoren C.F. Bastable, Publ ic Finance, 3rd ed. (London 1903), S. 611.

2) Die Analyse von Buchanan und Wagner bezieht sich auf die USA. Dieselbe Feststellung trifft Burton (1978), S. $31 \mathrm{ff}$. auch für den britischen Bereich.

3) Vgl. Buchanan/Wagner (1977), S. $11 \mathrm{ff}$.

4) Vgl. hierzu auch Buchanan/Wagner (1978b), S. 3, (1978c), S. 79 f. und (1978d), S. $13 \mathrm{f}$.

5) Zur Illustration dieses Wendepunkts auf wissenschaftlicher Ebene zitieren die Autoren (1977), S. 9 H. Dalton, der in den nachkeynesianischen Auflagen seiner "Principles of Public Finance" (1954), S. 221 schreibt: "We may now free ourselves from the old and narrow conception of balancing the budget, no matter over what period, and move towards the new and wider conception of balancing the whole economy."

6) Die einzelnen Glieder dieser Argumentationskette werden in den folgenden $A b-$ schnitten detailliert untersucht. Hier sei nur die Argumentation von Buchanan und Wagner kurz dargestellt. 
schaftlern, die im öffentlichen Interesse handeln, getroffen werden (wie KEYNES implizit unterstel1t) ${ }^{1}$ ), sondern von Politikern, die sich der Wiederwahlnotwendigkeit gegenübersehen und um die Stimmen der Wähler konkurrieren ${ }^{2)}$. Da jede Form der Besteuerung für die Wähler c.p. eine Nutzenminderung darstellt, jede Ausgabenerhöhung aber eine Nutzenerhöhung, werden Politiker, die mit Ausgabenprogrammen um Wählerstimmen konkurrieren, eher Erfolg haben als solche, die mit Steuererhöhungen oder Ausgabenkürzungen werben. Die beiden letzten Maßnahmen erhöhen zwar - so BW - ebenfalls den Nutzen vieler Wähler, da sie für zukünftig geringere Inflationsraten sorgen, doch werden diese zukünftigen Nutzengewinne von den Wählern aufgrund von Informationsmängeln nicht in vollem Umfang als Nutzengewinne wahrgenommen. Aus diesen Erwägungen folgt, daB Budgetdefizite den Wählern einen Nutzenzuwachs und damit den konkurrierenden Politikern Stimmen einbringen, Budgetüberschüsse aber das Gegenteil bewirken.

Während der klassische Grundsatz des Budgetausgleichs allzu ausgabenfreudigen Politikern eine Beschränkung auferlegte, weil der marginale Stimmengewinn einer Ausgabenerhöhung mit dem marginalen Stimmenverlust der gleichzeitig erforderlichen Steuererhöhung verglichen werden mußte ${ }^{3)}$, war es beim Fehlen einer derartigen Budgetbeschränkung möglich, die Ausgaben auszudehnen, ohne gleichzeitig die Steuern anzuheben. Ständig höher werdende Defizite waren somit die logische Folge der Anwendung keynesianischer Wirtschaftspolitik in einer Demokratie ${ }^{4}$ ) (vgl. Pfeile 1 und 2 in $A b-$ bildung 1 , S. 9).

1) Vgl. Buchanan/Wagner (1977), S. 77 f., (1978c), S. 84 f. und Burton (1978), S. $47 \mathrm{ff}$.

2) Vgl. Buchanan/Wagner (1977), S. $96 \mathrm{ff}$. Vgl. auch Buchanan (1954), wo die Gemeinsamkeiten und Unterschiede der politischen Konkurrenz mit der Konkurrenz auf dem Markt genauer analysiert werden.

3) Vgl. zu diesem Kalkül vor allem Downs (1957), S. 52 ff.

4) Vg1. hierzu Buchanan/Wagner (1978c), S. 87 ff. sowie Neumark (1949), S. 515, der die Bagatellisierung der Grenzen der Staatsschuld ebenfalls mit der keynesianischen Theorie in Verbindung bringt, aber auch Wildavsky (1980), S. 52 f. und Brunner (1975), S. 852, der den "New Economics" die Abschaffung der politischen Verantwortung für ein ausgeglichenes Budget vorwirft. 


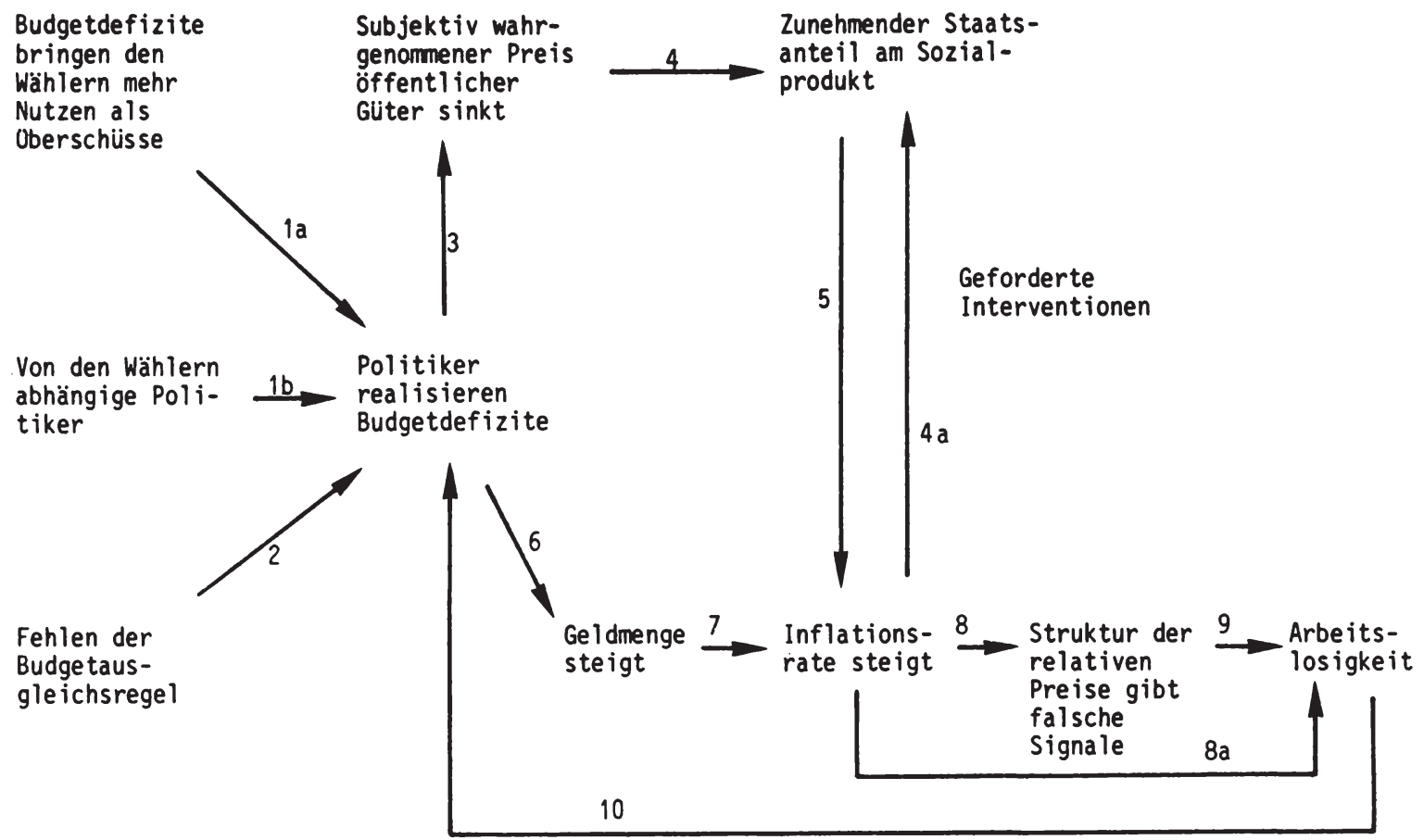

Abbildung 1: Die Struktur der neoinstitutionalistischen Argumentationskette 
Die so entstandenen Defizite haben nun wiederum zwei Effekte:

Zum einen senken sie den von den Wählern subjektiv wahrgenommenen Preis öffentlicher Güter $(P f e i 13)^{1)}$. Wenn beispielsweise bisher 100 Geldeinheiten (GE) für 10 Einheiten öffentlicher Guiter ausgegeben und durch 100 GE Steuern finanziert wurden, dann kostete eine Einheit des öffentlichen Gutes die Wähler $10 \mathrm{GE}^{2}$ ). Beschließen nun beispielsweise die Politiker zur Bekämpfung von Arbeitslosigkeit eine Steuersenkung in Höhe von 20 GE bei konstanten Staatsausgaben, dann müssen für die 100 Ausgabeneinheiten, mit denen weiterhin 10 Einheiten öffentlicher Güter bereitgestellt werden, nur noch $80 \mathrm{GE}$ an Steuern aufgebracht werden. M.a.W. der Preis für eine Einheit des öffentlichen Gutes sinkt auf $8 \mathrm{GE}$, was bei einer normal verlaufenden Nachfragekurve eine erhöhte Nachfrage nach öffentlichen Gïtern bewirkt. Diese erhöhte Nachfrage machen die Wähler über den politischen ProzeB geltend mit der Folge, daB der Anteil öffentlicher Güter an der Gesamtproduktion zunimmt. Auf diese Weise steigt der Staatsanteil am Sozialprodukt ${ }^{3}$ ) (Pfeil 4 ). Dieser wachsende Staatsanteil kann nach Ansicht von BW ${ }^{4}$ die Inflationsrate erhöhen (Pfeil 5 ), wenn

a) die Produktivität im Regierungssektor geringer ist als im privaten Sektor, was bei steigendem Staatsanteil die gesamtwirtschaftliche Produktivität senkt ${ }^{5}$, und

b) die Wachstumsrate der Geldmenge nicht entsprechend der Produktivitätsminderung gesenkt wird.

1) Auch verschiedene Formen der Besteuerung können diesen Effekt haben.

2) Hierbei wird unterstellt, daB im Ausgangszustand sowohl die Ausgaben und die Menge öffentlicher Güter als auch die Steuern von den Wählern in vollem Umfang subjektiv wahrgenommen werden.

3) Vgl. Buchanan/Wagner (1977), S. $103 \mathrm{ff}$.

4) Vgl. ebenda, S. 70

5) Vgl. hierzu Z.B. die empirische Untersuchung von Spann (1977). 
Die erhöhte Inflationsrate kann ihrerseits zu einem höheren Staatsanteil führen, wenn die Wähler anstelle des Staates gewinnmaximierende GroBunternehmen für die Inflation verantwortlich machen und staatliche Interventionen fordern (vgl. Pfeil 4a).

Für die Erhöhung der Inflationsrate führen BW allerdings noch einen weit gewichtigeren Grund an, und hiermit sind wir beim zweiten Effekt, den Defizite nach Ihrer Ansicht haben: sie bewirken eine Erhöhung der Geldmenge und damit eine Erhöhung der Inflationsrate (Pfeile 6, 7). Auch die Mitglieder einer de jure unabhängigen Zentralbank sind nach Ansicht von BW de facto dem Druck der offentlichkeit und der sie einsetzenden Politiker ausgesetzt. Sie erhöhen daher die Geldmenge im AusmaB der entstandenen Budgetdefizite ${ }^{1}$.

Diese Entwicklung ist ungefährlich, wenn das Defizit beschlossen wurde, um eine durch den Mangel an Gesamtnachfrage entstandene Beschäftigungskrise zu beheben ${ }^{2)}$. In dieser speziellen Situation, die KEYNES im Auge hatte, erhöht das deficit-spending über den Multiplikator den realen Output, und der erhöhten Geldmenge steht eine erhöhte Gütermenge gegenüber.

Ist die Beschäftigungskrise aber entstanden, weil die vorhandene Wirtschaftsstruktur nicht metir der vorhandenen Technologie-, Ressourcen- und Präferenzstruktur entsprach, dann verhindert eine derartige Politik gerade den vom Markt angezeigten Strukturwande ${ }^{3}$ ). Der durch das Defizit erhöhten Geldmenge steht keine äquivalente Gütermenge gegenüber, so daB die Preise bei einigen Gütern steigen. Aufgrund sinkender Reallöhne steigt dann zwar zunächst die Beschäftigung, aber es kommt gleichzeitig zu einer Verschiebung der

1) Vgl. Buchanan/Wagner (1977), S. $107 \mathrm{ff}$.

2) Vgl. ebenda, S. $164 \mathrm{f}$.

3) BW beziehen sich hier auf Hayek (1935). Vgl. Buchanan/Wagner (1977), S. 170, FuBnote 10 und (1978d), S. 26. Vgl. auch Wagner (1980), S. $16 \mathrm{ff}$. 
Struktur der relativen Preise, d.h. es werden Preissignale gegeben, die nicht mehr der zugrundeliegenden Präferenz-, Technologieund Ressourcenstruktur entsprechen ( $P f e i 18$ ). Durch Anpassung der Inflationserwartungen steigen schließlich die Reallöhne wieder an, so daß die Beschäftigungseffekte wieder rückgängig gemacht werden. (Pfeil 9) ${ }^{1}$. Will man die Beschäftigung aufrechterhalten, ist ein neuer, größerer Inflationsschub (bzw. ein neues Defizit) notwendig ( $v g l$. Pfeil 10) und der eben beschriebene ProzeB beginnt von neuem.

Die Wirtschaft kann nur dann endgiiltig zum Gleichgewicht zurückkehren, wenn sie eine temporäre Rezession durchmacht, die als inhärenter Bestandteil jeglicher wirtschaftlicher Entwicklung gesehen wird. Je länger man diese Rezession durch keynesianische Politik in einer nicht-keynesianischen ökonomischen Situation hinauszögert, desto länger und schwieriger wird der Weg zurück zum Gleichgewicht. "There is no costless cure for a maladjusted economy. Reallocation of capital and labour must take place before the economy's structure of production will once again reflect the underlying data to which a free economy adapts. . Recession is an inherent part of the recovery process."2) Abbildung 1 illustriert die Struktur der neoinstitutionalistischen Argumentation.

Im folgenden wird zunächst die Frage untersucht, ob bzw. unter welchen Annahmen es in einer Demokratie zur Bildung wachsender Budgetdefizite kommen kann (Pfeile 1 und 2). Dabei ist, auch als Grundlage der Analyse in Abschnitt IV, neben dem EinfluB von Defiziten der Einfluß alternativer Steuerinstitutionen und Organisa-

1) Verschärft werden die Beschäftigungsprobleme durch die inflationsbedingte Verunsicherung der Wirtschaft, insbesondere bei der Preiskalkulation, durch die Besteuerung inflationsbedingter Scheingewinne und den dadurch bedingten beschränkten Finanzierungsspielraum der Unternehmen (Pfeil 8a). Vgl. Buchanan/Wagner (1977), S. $66 \mathrm{f}$.

2) Buchanan/Wagner (1977), S. 170. Vgl. zu diesem Punkt auch Wagner (1977), $\mathrm{S} .405 \mathrm{f}$. Die Argumentation weist starke Ahnlichkeiten zur monetaristischen Position auf. Vgl. Z.B. Brunner (1970a), S. 75 und Buchanan/Wagner (1978), S. 635. Zu beachten ist in diesem Zusammenhang auch die Erwähnung einer natuirlichen Arbeitslosenquote. Vgl. Buchanan/Wagner (1977), S. 168 
tionsformen des politischen Entscheidungsprozesses auf den subjektiv wahrgenommenen Preis öffentlicher Güter zu untersuchen (Pfeil 3).

Abschnitt IV diskutiert die Auswirkungen stabilitätspolitisch bedingter Defizite auf den allokativen Bereich (Pfeil 4), und $A b-$ schnitt $V$ setzt sich kritisch mit der neoinstitutionalistischen Behauptung auseinander, die Zentralbank erhöhe die Geldmenge im Ausma $B$ der Budgetdefizite und trage dadurch zu einer Erhöhung der Inflationsrate bei (Pfeile 6 und 7 ). 


\section{DEMOKRATIE UND DEFIZITE}

1. Theoretische Analyse der Kosten und Nutzen von Budgetuberschüssen und Budgetdefiziten unter alternativen Bedingungen

In diesem Abschnitt soll zunächst dargestellt werden, wie sich Budgetüberschüsse und Budgetdefizite nach Ansicht von BW auf das Nutzenniveau der Wähler auswirken. Die Untersuchung erfolgt getrennt für den (keynesianischen) Fall der Unterbeschäftigung und den (nicht-keynesianischen) Fall der Vollbeschäftigung (vgl. Abschnitt III.1.1. und III.1.2.).

Die Wähler werden versuchen, im politischen ProzeB diejenige Budgetalternative geltend zu machen, die ihnen einen höheren Nettonutzen verspricht. Ob sich der von den wählern ausgeübte politische Druck auf die praktische Wirtschaftspolitik auswirkt, hängt davon ab, ob diese Politik von einer unabhängigen Elite bestimmt wird oder von demokratisch gewählten Politikern, die sich der Wiederwahlnotwendigkeit gegenübersehen und die Wünsche der Wähler berücksichtigen müssen (vg1. Abschnitt III.1.3.). Trifft letzteres zu, dann hängt die Auswirkung des politischen Prozesses auf den Budgetsaldo davon ab, ob die Politiker bei ihren Budgetentscheidungen die Budgetausgleichsregel beachten müssen oder nicht. Die anschließenden Abschnitte III.1.4. und III.2. werden sodann der Kritik der neoinstitutionalistischen Analyse gewidmet.

\subsection{Kosten und Nutzen marginaler Budgetüberschüsse ${ }^{1)}$}

Die Erwirtschaftung eines marginalen Budgetüberschusses setzt Steuererhöhungen und/oder Ausgabenkürzungen voraus. Diese Maßnahmen machen zumindest einen Teil der Wähler zu Verlierern, sei es, daB aufgrund der Steuererhöhung ihr verfügbares Einkommen verringert

1) Vgl. zu den Abschnitten III.1.1. und III.1.2. Buchanan/Wagner (1977), S. $99 \mathrm{f.},(1978 \mathrm{c})$, S. $87 \mathrm{ff}$. und (1978d), S. $19 \mathrm{ff}$. 
wird, sei es, daB aufgrund der Ausgabenkürzung weniger öffentliche Güter bereitgestellt werden. Ein marginaler BudgetüberschuB führt also zu einem Rückgang des Gegenwartskonsums und daher zu einer Nutzenminderung bei einigen Wählern. In einer Situation der Unterbeschäftigung ${ }^{1}$ hätte ein marginaler Budgetüberschuß überdies einen kontraktiven Multiplikatoreffekt und damit für die Wähler weitere Nutzenminderungen zur Folge. Es bleibt also festzuhalten, daB Budgetüberschüsse in einer keynesianischen ökonomischen Situation für die Wähler mit Nutzenminderungen verbunden sind und daher abgelehnt werden.

Etwas anders sind Budgetüberschüsse in Zeiten der Vollbeschäftigung oder steigender Inflationsraten zu beurteilen. In diesen nichtkeynesianischen ökonomischen Situationen verhindert ein marginaler BudgetüberschuB ansonsten mögliche Preissteigerungen und hat daher Nutzengewinne zur Folge, welche von den Wählern mit den vorher erwähnten Nutzenminderungen verglichen werden. BW behaupten, daB sich für die Wähler netto eine Nutzenminderung ergibt, und zwar aus folgenden Gründen: Die Steuererhöhungen bzw. Ausgabenkürzungen bewirken einen direkt wahrgenommenen Konsumverzicht in der Gegenwart. Die Nutzengewinne in Form von geringeren Inflationsraten liegen dagegen in der Zukunft und werden nur indirekt wahrgenommen: "The benefit side of a budget surplus is not directly experienced but rather must be imagined. It takes the form of the hypothetical or imagined gains from avoiding what would otherwise have been an inflationary experience ${ }^{2)}$." Das Individuum ist nicht exakt daruber informiert, wie es von einer zukünftigen Inflation persönlich betroffen wird. Es hat also nicht nur die Aufgabe, zukünftige Nutzen auf den Gegenwartswert abzudiskontieren, es muB zusätzlich die Auswirkungen eines möglichen makroökonomischen Effekts (über die sich

1) Im folgenden wird die Situation der Unterbeschäftigung in Anlehnung an Buchanan/Wagner als "keynesianische ökonomische Situation" bezeichnet, während Situationen der Vollbeschäftigung und der Inflation als "nicht-keynesianische ökonomische Situationen" bezeichnet werden.

2) Buchanan/Wagner (1978d), S. 21 
nicht einmal die okonomen einig sind) auf sein persönliches (mi-

kroökonomisches) Nutzenniveau abschätzen ${ }^{1)}$. Aufgrund dieser informationsbedingten und psychologischen Faktoren werden die zukünftigen Nutzengewinne von den Individuen nach Meinung von $B W$ unterschätzt, so daB Budgetüberschüsse auch in einer nicht-keynesianischen ökonomischen situation abgelehnt werden.

\subsection{Kosten und Nutzen marginaler Budgetdefizite}

Ein marginales Budgetdefizit bedeutet Steuersenkungen und/oder Ausgabenerhöhungen. Zumindest für einige Wähler ents.tehen hieraus Nutzengewinne durch höhere verfügbare Einkommen und/oder Mehrkonsum von öffentlichen Gütern. In der keynesianischen Unterbeschäftigungssituation kommen Nutzengewinne durch die positiven Beschäftigungseffekte der Defizite hinzu, so daß in dieser Situation Defizite eindeutig die Zustimmung der Wähler finden. In einer nichtkeynesianischen ökonomischen Situation muB die Möglichkeit berücksichtigt werden, daB Budgetdefizite die Inflationsrate erhöhen. Aus den vorher erwähnten Gründen werden jedoch die damit verbundenen Nutzenminderungen von den Wählern nicht voll berücksichtigt, so daB BW auch in nicht-keynesianischen ökonomischen Situationen von einem Nettonutzengewinn ausgehen.

\subsection{Die Auswirkungen alternativer politischer Voraussetzungen auf den Budgetsaldo}

Im folgenden wird untersucht, welcher Budgetsaldo angesichts der im vorigen Abschnitt herausgearbeiteten Kosten- und Nutzeneffekte be $i$ alternativen politischen Institutionen resultiert. Dabei wird aus Gründen der Anschaulichkeit dem Extrem des allwissenden, immer im öffentlichen Interesse handelnden Politikers das andere Extrem des völlig eigennützigen, aber von den augenblicklichen Wählerwünschen abhängigen Politikers gegenübergestellt.

1) Hinzu kommt, daB niemand weiB, wie die Situation ohne die StabilisierungsmaBnahme ausgesehen hätte. 
Im letzteren Modell ist der Politiker lediglich dazu da, die augenblicklichen Wählerwünsche auszuführen, d.h. Wir haben den theoretischen Grenzfall vor uns, in dem Politiker unter den Bedingungen der vollkommenen Konkurrenz um Wählerstimmen konkurrieren ${ }^{1)}$. Das bedeutet auch, daB lediglich die Nachfrage nach öffentlichen Gütern berücksichtigt wird $\left.{ }^{2}\right)$. Die Angebotsseite und damit die mit staatlichen Bürokratien verbundenen Probleme werden bewuBt vernachlässigt, um die Argumente von $B W$ exakt herauszuarbeiten.

\subsubsection{Die Möglichkeit einer "symmetrischen" Politik bei Vorliegen der "Presuppositions of Harvey Road" 3 )}

KEYNES ging bei seinen wirtschaftspolitischen Empfehlungen implizit davon aus, daB Wirtschaftspolitik von einer kleinen Gruppe aufgeklärter Politiker (einer Elite) betrieben werde, die unabhängig ist und im öffentlichen Interesse hande $1 t^{4) 5}$ ). Unter diesen Voraussetzungen zeichnet sich folgende Budgetpolitik im Konjunkturverlauf $a b$ : In den Zeiten der Unterbeschäftigung wird die Nachfrage durch deficit-spending erhöht, bis die Vollbeschäftigung erreicht ist. In Boom-Phasen verlangen die Wähler zwar ebenfalls Defizite, weil sie die negativen Folgen der Inflation nicht voll berücksichtigen. Die herrschende, sachverständige Elite sieht aber diese negativen Folgen voraus und betreibt daher im öffentlichen Interesse eine kontraktive Politik der Budgetüberschüsse. Unter der Annahme, daß Rezessions- und Boom-Phasen gleich lang und gleich intensiv sind, ergibt sich über den Konjunkturzyklus hinweg betrachtet ein ausgeglichenes Budget. Unter den obengenannten politischen Vorausset-

1) Im Ergebnis kommt dies der Annahme der direkten Demokratie gleich.

2) Vgl. auch Roberts (1978), S. $604 \mathrm{f}$.

3) Der Begriff "presuppositions of Harvey Road" stammt von R. Harrod und steht für die Einstellung von Keynes gegenüber dem politischen ProzeB, welche im folgenden wiedergegeben wird. Vgl. Harrod (1951), S. 192 f. In der Harvey Road befand sich der Wohnsitz von J.M. Keynes.

4) Vgl. Buchanan/Wagner (1977), S. $78 \mathrm{ff}$. und (1978c), S. $84 \mathrm{f}$. sowie Rose/Peters (1978), S. $139 \mathrm{ff}$.

5) Zumindest war er der Auffassung, daB die ökonomischen Berater genügend Oberzeugungskraft aufbringen würden, um die Politiker zu einer Politik im öffentlichen Interesse zu veranlassen. 
zungen wird demnach eine "symmetrische" Budgetpolitik betrieben. Die Defizite der Rezessionsphasen werden durch Oberschüsse in den oberhitzungsphasen ausgeglichen. Es erfolgt keine Abkehr vom klassischen Prinzip des Budgetausgleichs, sondern lediglich eine Verlängerung der betrachteten Budgetperiode ${ }^{1 \text { ). }}$.

\subsubsection{Der Budgetsaldo in einer Demokratie ${ }^{2}$ )}

Wird die Budgetpolitik dagegen von demokratisch gewählten Volksvertretern bestimmt, die ihren eigenen Nutzen unter der Nebenbedingung der Wiederwahlnotwendigkeit maximieren, so werden diese eine Steuer/Ausgaben-Kombination vorschlagen, die ihnen die Mehrheit der Wählerstimmen sichert. Genauer gesagt: Es wird derjenige Budgetsaldo realisiert, der den Nutzen des Medianwähzers maximiert ${ }^{3)}$. Ordnet man die von allen Wählern gewünschte Höhe des Budgetsaldos der Reihe nach, so wird bei Entscheidungen mit einfacher Mehrheit derjenige Budgetsaldo realisiert, den der in der Mitte dieser Reihe stehende Wähler (der Medianwähler) präferiert. Abbildung 2 veranschaulicht den Zusammenhang für den Fall von sieben Wählern:

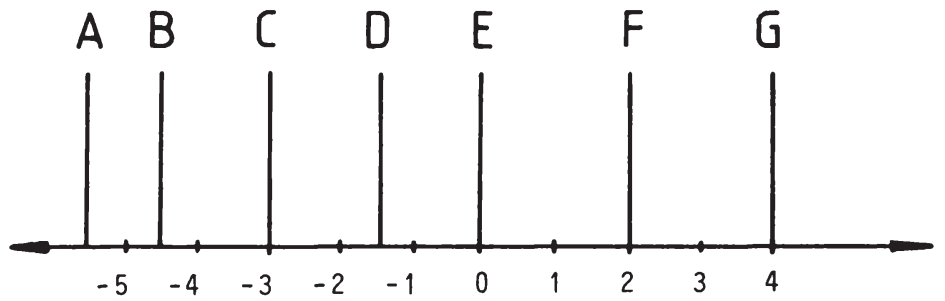

Abbildung 2: Der Budgetsaldo in einer Demokratie

1) Vgl. Buchanan/Wagner (1977), S. 95

2) $\mathrm{Vgl}$. ebenda, S. $96 \mathrm{ff}$.

3) Vgl. Black (1948), S. $23 \mathrm{ff}$. 
Während $E$ für ein ausgeglichenes Budget stimmt, verlangen $F$ und $G$ einen BudgetuiberschuB, A, B, C und D dagegen ein Budgetdefizit. Wird nun, beginnend mit dem Vorschlag G, über die Vorschläge paarweise abgestimmt, so wird $D$ gewinnen, weil er als Medianwähler der einzige ist, der eine Minderheit in eine Mehrheit verwandeln kann ${ }^{1}$.

Bei Zugrundelegung der Annahmen aus den Abschnitten III.1.1. und III.1.2. wird sich der Medianwähler in sämtlichen Konjunktursituationen im Defizitbereich befinden, so daB unter nicht-keynesianischen politischen Voraussetzungen eine Wählertendenz zu Budgetdefiziten besteht. Für. Politiker in einer Demokratie besteht daher ein Anreiz, in sämtlichen ökonomischen Situationen Defizite zu machen. Die Frage, ob sie diese defizitäre Budgetpolitik tatsächlich betreiben können, hängt von den Rege $n$ ab, denen sich die Politiker be $i$ ihren Budgetentscheidungen unterwerfen müssen. Ist ihnen die Regel des Budgetausgleichs vorgegeben, dann können die Politiker trotz ihrer Neigung zu Defiziten keine Defizitpolitik betreiben. Zeichnet sich im Verlauf eines Haushaltsjahres ein potentielles Budgetdefizit ab, so müssen unbeachtlich der Wünsche der Wähler entweder die Steuern erhöht oder die Ausgaben geküzt werden. Erst wenn die Regel des Budgetausgleichs aufgehoben wird - und dies ist durch die Verbreitung des Keynesianismus geschehen - wirkt sich die Neigung der Politiker, den Wünschen der Wähler entsprechend Defizite zu machen, auf den tatsächlichen Budgetsaldo aus.

Diese für den theoretischen Grenzfall der vollständigen Kankurrenz zwischen Politikern abgeleitete Entwicklung wird natürlich in der Realität nicht mit dieser Eindeutigkeit auftreten. Zahlreiche Marktunvollkommenheiten auf dem Markt für politische Unternehmer ${ }^{2}$ )

1) $F$ wird zunächst mit $6: 1$ Stimmen gegen $G$ gewinnen, dann $E$ gegen $F$ mit $5: 2$ und schlieBlich $D$ gegen $E$ mit $4: 3$. C wird dagegen dem Vorschlag $D$ mit $3: 4$ Stimmen unterliegen. Die Probleme zyklischer Majoritäten werden in diesem Zusammenhang vernachlässigt. Vgl. hierzu Z.B. Frey (1977), S. 94 ff.

2) Zu denken ist vor allem an die Tatsache, daß fast alle westlichen Staaten repräsentative Demokratien sind, in denen nur alle vier bis fünf Jahre gewählt wird. 
entheben den Politiker von der laufenden direkten Kontrolle durch die Wähler. Vor allem beim Herannahen des Wahltermins müssen Politiker jedoch Wählerwünsche in gewissem Umfang berücksichtigen, so da $B$ mit der aufgezeigten Entwicklung zumindest in der Tendenz gerechnet werden muß.

\subsection{Kritik der theoretischen Analyse}

\subsubsection{Vorgehenswe ise}

Zur Oberprüfung der BW-These, in demokratischen Staaten würden bei Fehlen der Budgetausgleichsregel Budgetdefizite realisiert, wird im folgenden ein allgemeines Modell mit anfangs noch nicht näher spezifizierten funktionalen Abhängigkeiten aufgestellt. AnschlieBend wird untersucht, wie diese funktionalen Abhängigkeiten nach Ansicht von $B W$ beschaffen sind und welche Konsequenzen für den Budgetsaldo hieraus resultieren (vgl. die Abschnitte III.1.4.2. und III.1.4.3.). Der. Diskussion der von BW unterstellten Wahrnehmungsasymmetrien (Abschnitte III.1.4.4. und III.1.4.5.) folgt eine Analyse alternativer Wirkungen von Budgetsalden auf Realeinkommen und Inflationsrate (Abschnitt III.1.4.6.). Weiterhin werden von BW nicht berücksichtigte Effekte marginaler Budgetsalden in die Betrachtung einbezogen (Abschnitte III.1.4.7. bis III.1.4.10.). Dem Fazit der theoretischen Argumente schlieBt sich eine oberprüung der BW-These anhand politometrischer Schätzungen an (Abschnitt III.2.).

\subsubsection{Ein einfaches Mode 11}

Der Nutzen $U^{i}$ eines Wählers $i$ hänge von seinem Realeinkommen und von der Menge der von ihm konsumierten öffentlichen Güter ab. Dabei spielt allerdings sein tatsächliches Realeinkommen $y^{i} / p$ und sein tatsächlicher Konsum öffentlicher Güter $x^{i}$ eine untergeordnete Rolle. Entscheidend ist das von ihm wahrgenommene ${ }^{1)}$ Real-

1) Vgl. Buchanan/Wagner (1977), S. 128 
einkommen $Y^{i} / P^{i}$ und der von ihm wahrgenommene Realkonsum öffentlicher Güter $x^{i}$. Die GröBe $\gamma^{i}$ repräsentiert weitere, durch $Y^{i} / P^{i}$ und $x^{i}$ nicht erfaßte Faktoren, die das Nutzenniveau des Wählers $i$ beeinflussen. Somit ergibt sich die individuelle Nutzenfunktion

(III.1) $\quad U^{i}=U^{i}\left(\gamma^{i} / P^{i}, x^{i}, \gamma^{i}\right)$.

Die Argumente der Nutzenfunktion sind dabei als Gegenwartswerte aller gegenwärtig wahrgenommenen und zukünftig erwarteten Realeinkommens- und -konsumströme anzusehen, d.h. es gilt

$(\text { III.2 })^{1)} \quad \frac{y^{i}}{P^{i}}=\sum_{t=0}^{n} \frac{y_{t}^{i} / p_{t}^{i}}{\left(1+r^{i}\right)^{t}}$

(III.3) $\quad x^{i}=\sum_{t=0}^{n} \frac{x_{t}^{i}}{\left(1+r^{i}\right)^{t}}$.

Die tatsächlichen Größen $y^{i}$, $p$ und $x^{i}$ seien abhängig vom Budgetsaldo der Gegenwart $B_{0}$, die wahrgenommenen Größen von $B_{0}$ und vom Informationsniveau des Wählers $m^{i}$. Somit gelten die folgenden Beziehungen:

(III.4) $\quad y_{t}^{i}=y^{i}\left(B_{0}\right) \quad$ und damit

(III.4a $)^{2)} \quad Y_{t}^{i}=Y^{i}\left(B_{0}, m^{i}\right)$;

(III.5) $\quad p_{t}=p\left(B_{0}\right) \quad$ und damit

(III.5a) $\quad P_{t}^{i}=P^{i}\left(B_{0}, m^{i}\right)$;

1) $r^{i}$ bezeichnet die individuelle Diskontrate, die durch die Zeitpräferenz des Wählers $i$ bestimmt ist.

2) (III.4a) folgt aus der Bezeichnung $Y_{t}^{i}=g^{i}\left(y^{i}\left(B_{0}\right), m^{i}\right)$. 
( I I . 6) $\quad x^{i}=x^{i}\left(B_{0}\right)$

und damit

(III.6a) $\quad x_{t}^{i}=x^{i}\left(B_{0}, m^{i}\right)$.

Wie sich durch Lösung des Gleichungssystems leicht erkennen läBt, ist der Nutzen eines Wählers vom Budgetsaldo der Gegenwart, vom Informationsniveau des Wählers und von $\gamma^{i}$ abhängigl):

(III.1a) $u^{i}=f^{i}\left(B, m^{i}, \gamma^{i}\right)$.

Aus dieser Gleichung läBt sich der vom Wähler $i$ gewünschte Budgetsaldo $B^{i}$ ermitteln. Unter Berücksichtigung von (III.4) bis (III.6) und (III.4a) bis (III.6a) ergibt sich

(III.1b) $\quad B^{i}=B^{i}\left(y^{i} / p, x^{i}, m^{i}, \gamma^{i}\right)$.

Zur Oberprüfung der BW-These nehmen wir wie im vorigen Abschnitt $a n$, der von der Regierung realisierte Budgetsaldo $B^{r}$ entspreche dem vom Medianwähler gewünschten Saldo.

(III.7) $\quad B^{r}=B^{M}\left(y^{M} / p, x^{M}, m^{M}, \gamma^{M}\right)$.

Somit hängt der in einer Demokratie bei Fehlen der Budgetausgleichs regel realisierte Budgetsaldo von $\gamma^{M}$ und den folgenden Faktoren ab:

a) von der Wirkung marginaler Budgetsalden auf das gegenwärtige und zukünftige Realeinkommen des Medianwählers M:

$($ III.8) 2$) \quad \frac{\partial\left(y_{t}^{M} / p_{t}\right)}{\partial B}$ $t=1,2, \ldots, n$,

d.h. genauer von der Wirkung marginaler Budgetsalden auf das gegenwärtige und zukünftige Nominaleinkommen des Medianwählers

1) Der Zeitindex 0 wird aus Gründen der Obersichtlichkeit weggelassen.

2) Im Falle eines marginalen Budgetüberschusses ist die Anderung von B positiv, im Falle eines marginalen Defizits negativ. 
und auf das gegenwärtige und zukünftige Preisniveau. MaBgeblich sind demnach die Ausdrücke

(III.9) $\frac{\partial y_{t}^{M}}{\partial B}$

$$
t=1,2, \ldots, n
$$

und

$(\text { III.10 })^{1)} \quad \frac{\partial p_{t}}{\partial B}$

$$
t=1,2, \ldots, n
$$

b) von der Wirkung marginaler Budgetsalden auf die vom Medianwähler in Gegenwart und Zukunft konsumierte Menge öffentlicher Güter

(III.11) $\frac{\partial x_{t}^{M}}{\partial B}$ $t=1,2, \ldots, n$

c) von der Zeitpräferenz des Medianwählers, die sich in $r^{M}$ ausdrückt;

d) vom Informationsniveau $m^{M}$ des Medianwählers und seinen noch nicht näher spezifizierten Bestimmungsgründen.

Im folgenden Abschnitt werden nun die von BW angenommenen und die zur Unterstützung ihrer These zusätzlich notwendigen Spezifizierungen dieser Faktoren untersucht.

\subsubsection{Der Budgetsaldo unter neoinstitutionalistischen Annahmen}

BW gehen davon aus, daB marginale Budgetdefizite das gegenwärtige Realeinkommen und/oder den gegenwärtigen Konsum öffentlicher Güter bei zunächst konstantem Preisniveau erhöhen, in späteren Situatio-

1) Der Ausdruck (III.8) ergibt $\left[\left(\partial y_{t}^{M} / \partial B\right) p_{t}-\left(\partial p_{t} / \partial B\right) y_{t}^{M}\right] / p_{t}^{2}$, so daB die partiellen Differentialquotienten (III.9) und (III.10) das Vorzeichen von (III.8) bestimmen. 
nen jedoch zu Preissteigerungen führen. Es kommt somit lediglich zu einer temporären Erhöhung des Realeinkommens ${ }^{1)}$. Marginale Budgetüberschüsse senken dagegen das Gegenwartseinkommen und verhindern mögliche Preissteigerungen in der Zukunft. Gehen wir der Einfachheit halber von einer Zwei-Perioden-Betrachtung aus, so haben unter diesen Annahmen die im vorigen Abschnitt genannten Ausdrücke (III.8) bis (III.11) folgende Vorzeichen. In der Gegenwart ( $t_{0}$ ) gilt für die Realeinkommenswirkung

(III.8.0) $\frac{\partial\left(y_{0}^{M} / p_{0}\right)}{\partial B}<0$,

wobe $i$

(III.9.0) $\quad \frac{\partial y_{0}^{M}}{\partial B}<0$

und

(III.10.0) $\quad \frac{\partial p_{0}}{\partial B}=0$.

Für die Wirkung des Budgetsaldos auf den Konsum öffentlichter Güter gilt

(III.11.0) $\frac{\partial x_{0}^{M}}{\partial B}<0$.

Für die Zukunft $\left(t_{1}\right)$ gilt entsprechend

(III.8.1) $\frac{\partial\left(y_{1}^{M} / p_{1}\right)}{\partial B} \geqq 0$,

1) Das zukünftige Realeinkommen kann sogar geringer sein als im Ausgangszustand. 
wobe $i$

(III.9.1) $\frac{\partial y_{1}^{M}}{\partial B} \geqq 0$

und

(III.10.1) $\frac{\partial p_{1}}{\partial B}<0$.

Ober die zukünftige Versorgung mit öffentlichen Gütern machen BW keine Aussagen. Wir nehmen

(III.11.1) $\frac{\partial x_{1}^{M}}{\partial B}=0$

an, obwohl es möglich ist, daB ein durch ein Defizit im Zeitpunkt $t=0$ bereitgestelltes öffentliches Investitionsgut in der Zukunft noch Nutzungen abgibt ${ }^{1}$ ). Weitere makroökonomische Effekte werden von BW nicht berücksichtigt, d.h. $\gamma^{M}=0$.

Auf die Zeitpräferenzen der Wähler gehen BW nicht ausführlich ein. In ihrer Schrift "Democracy in Deficit" behaupten sie jedoch, daB die durch Defizite verursachte Inflation die Wirtschaftssubjekte verunsichere und zu einer Verkürzung des Zeithorizonts führe ${ }^{2}$ ). Es erscheint somit gerechtfertigt, von einer starken Gegenwartspräferenz des Medianwählers auszugehen ${ }^{3)}$.

1) Das Vorzeichen von (III.11.1) hängt also genau genommen von der Art der bereitgestellten (bzw. aufgrund eines oberschusses nicht bereitgestellten) öffentlichen Güter im Zeitpunkt $t_{0} a b$.

2) Vgl. Buchanan/Wagner (1977), S. 65

3) An anderer Stelle ((1977), S. 100 und (1978d), S. 21) sprechen Buchanan/Wagner von einem psychologischen Uniterschied der Kosten- und der Nutzenseite von Budgetuiberschüssen. 01 son (1978), S. 108 spricht in seiner Interpretation von "psychologischen Asymmetrien", die zu den noch zu erörternden "Informationsasymmetrien" hinzukommen. Auch diese Ausführungen legen nahe, daB Buchanan/ Wagner von einer starken Gegenwartspräferenz der Wähler ausgehen. 
SchlieBlich unterstellen $B W$, da $B$ in der Gegenwart auftretende Effekte stärker wahrgenommen werden als zukünftige, wofür neben den eher den Zeitpräferenzen zuzuordnenden psychologischen Effekten sogenannte "Informationsasymmetrien" 1 ) verantwort 1 ich sind.

Der Grund dafür, daß die Wähler über die Gegenwart besser informiert sind als über die Zukunft, liegt nach Ansicht von BW darin, da $B$ in der Gegenwart auftretende Effekte von Budgetsalden die ökonomische Position jedes Individuums direkt beeinflussen (sie werden "am eigenen Leibe verspürt"), während sich zukünftige Effekte von Budgetsalden nur indirekt auf das Nutzenniveau der Wähler auswirken. Budgetsalden in der Gegenwart haben bestimmte makroökonomische Effekte in der Zukunft, und jeder Wähler muB eine Vorstellung darüber entwickeln, wie sich diese makroökonomischen Effekte auf sein persönliches Nutzenniveau auswirken. Daher sind die Opportunitätskosten für Informationen über zukünftige Effekte von Budgetsalden auf das Nutzenniveau höher als für Informationen über die in der Gegenwart "direkt" wahrgenommenen Effekte.

Unter den genannten Annahmen erscheint es plausibel, daB sich der Medianwähler für ein Budgetdefizit ausspricht. Der Grund hierfür liegt darin, daB die nutzensteigernden Effekte von Budgetdefiziten in der Gegenwart, die nutzenmindernden in der Zukunft anfallen. Letztere werden dann aufgrund hoher opportunitätskosten der Informationen über die Zukunft in geringerem Umfang wahrgenommen als gegenwärtige Effekte und aufgrund der starken Gegenwartspräferenz mit einem hohen Diskontfaktor abdiskontiert.

Nun sind BW keineswegs die ersten Okonomen, die mit der Minderschätzung der Zukunft argumentieren. So hat VON BOHM-BAWERK schon Anfang dieses Jahrhunderts die Minderschätzung des Zukunftskonsums als Begründung für einen positiven Zins angeführt ${ }^{2}$. Eine Minder-

1) $\mathrm{Vgl} .01$ son (1978), S. 108

2) Vgl. von Böhm-Bawerk (1921), S. 477 ff. sowie Beetz (1978), S. 93 f. 
schätzung der Zukunft ist aber für die von BW behauptete Tendenz zu Defiziten ebensowenig hinreichend wie das angefuhrte Informationsproblem. Ein Unternehmer kann eine starke Präferenz für die Gegenwart haben und gleichwohl investieren, wenn der Verzicht auf gegenwärtige Erträge Zukunftserträge erbringt, deren abdiskontierter Gegenwartswert höher ist als der der Gegenwartserträge. Weiterhin wird ein Konsument trotz Gegenwartspräferenz bereit sein, auf einen Teil seines Gegenwartskonsums zu verzichten, wenn ihm dieser Verzicht einen ausreichend hohen Zukunftskonsum verspricht ${ }^{1}$ ).

Die BW-These trifft also trotz der obengenannten Annahmen nicht zu, wenn Budgetüberschüsse dem Medianwähler ausreichend hohe Zukunftserträge versprechen. Dies ist in einem Staat wie der Bundesrepublik Deutschland, dessen ältere Bürger bereits mit 2 wei Hyperinflationen und ihren Folgen konfrontiert wurden, nicht unwahrscheinlich. Eine aufgrund dieser Erfahrungen stark ausgeprägte Anti-Inflations-Mentalität der Wähler könnte dazu führen, daB der Medianwähler Budgetüberschüsse bevorzugt ${ }^{2}$ ).

Die Frage der Inflationsbewertung durch die Wähler kann letztendiich nur empirisch entschieden werden. In Abschnitt III.2. wird daher versucht, anhand empirischer Untersuchungen AufschluB darüber zu erhalten, wie die Wähler die Erreichung des ziels der Preisniveaustabilität bzw. alternativer wirtschaftspolitischer Ziele bewerten. Vorher scheinen jedoch noch einige oberlegungen zur theoretischen Analyse von BW angebracht. Dabei geht es in erster Linie um einige Ergänzungen zu den von den Neoinstitutionalisten postulierten Informationsasymmetrien und um die Berücksichtigung alternativer Effekte von Budgetsalden.

1) Diese Kalküle sind den Lehrbüchern zur Kapital-bzw. Wohlfahrtstheorie zu entnehmen. Vgl. Z.B. Fisher (1932), S. $126 \mathrm{ff}$. und Hirshleifer (1974), S. $46 \mathrm{ff}$.

2) Vgl. hierzu vor allem die Bemerkungen zu psychologischen Grenzen der Staatsverschuldung in III.1.4.10., aber auch Blackaby (1979), S. 196 f., der behauptet, im Vereinigten Königreich würde eine Verminderung der Inflationsrate den Politikern höhere Stimmengewinne einbringen als eine Senkung der Arbeitslosenquote. 
1.4.4. Einige Bemerkungen zum Informationsproblem

Das Nutzenniveau eines Wählers wird gemäß (III.1) nicht von objektiven Einkommens - und Konsumgrößen tangiert, sondern von den vom Wähler subjektiv wahrgenommenen Größen.

Daher kommt dem Informationsniveau des Wählers eine entscheidende Bedeutung $z u^{1)}$ und es bietet sich an, die Determinanten des Informationsniveaus etwas exakter zu untersuchen.

Ein rationaler Wähler wird sein optimales Informationsniveau durch Gleichsetzen der Grenzkosten mit dem Grenznutzen der Informationsgewinnung bestimmen ${ }^{2}$.

Nun kann man sicherlich davon ausgehen, daß Informationen über in der Gegenwart direkt empfundene Effekte von Budgetsalden zu geringeren Kosten erhältich sind als Informationen über die Zukunft, die ja immer mit Unsicherheit behaftet sind.

Wird jedoch berücksichtigt, daß Informationen nicht um ihrer selbst willen angestrebt werden, sondern deshalb, weil sie den Nutzen mittelbar - zum Beispiel durch eine aufgrund besserer Information ermöglichten Erhöhung des Realeinkommens - steigern, so wird deutlich, daB der Grenznutzen der Information und damit das optimale Informationsniveau über Gegenwart und Zukunft nicht nur vom objekt der Informationsgewinnung, sondern auch von der Zeitpräferenz des informationssuchenden wählers abhängt ${ }^{3)}$.

1) Bartlett (1973), insbesondere Kapitel 3, bezeichnet den Nutzen der Individuen aufgrund dieser oberlegung als endogen und durch gezielte Informationspolitik der Politiker beeinflukbar.

2) Vgl. auch Meckling (1978), S. 102

3) Die Informationskosten und damit das Informationsniveau werden von den herrschenden Steuerinstitutionen entscheidend mitbestimmt. Vgl. auch Buchanan (1967), S. 8 und Bartlett (1973), S. 92 f. Dieser Effekt wird in III.1.4.5. genauer untersucht und an dieser Stelle vernachlässigt, da hier lediglich der EinfluB der Zeitpräferenz auf das optimale Informationsniveau aufgezeigt werden soll. 
Formal läßt sich dies recht einfach zeigen: Betrachten wir den Nutzen der Information über einen bestimmten Zeitpunkt $\tau$ als vom Realeinkommen $y_{\tau}$ abhängig, das sich aufgrund dieser Information zum Zeitpunkt $\tau$ erzielen läßt, so gilt:

(III.12) $\quad U_{\tau}=U_{\tau}\left[y_{\tau}\left(m_{\tau}\right)\right]$,

woraus sich die Grenznutzenfunktion

(III.13) $\frac{d U_{\tau}}{d m_{\tau}}=\frac{\partial U_{\tau}}{\partial y_{\tau}} \cdot \frac{\partial y_{\tau}}{\partial m_{\tau}}$

ableiten 1äBt. Der Grenznutzen der Information ist daher zum einen davon abhängig, wieviel Realeinkommen eine infinitesimal kleine Informationseinheit über den Zeitpunkt $\tau$ einbringt, zum anderen vom Grenznutzen des Einkommens zum Zeitpunkt $\tau$.

Somit wird das Informationsniveau über Gegenwart und Zukunft nicht nur durch die Kosten der Informationsgewinnung, sondern auch durch die Zeitpräferenz des informationssuchenden Wählers bestimmt. Weist somit ein Wähler - abweichend von der Ansicht von BW - eine starke Zukunftspräferenz auf, so wird er über die Zukunft besser informiert sein und die zukiunftigen Effekte von Budgetsalden werden bei seiner Entscheidung über Defizite versus Oberschüsse eine größere Rolle spielen als von BW angenommen.

Bei einigen Autoren ${ }^{1)}$ findet sich die These, die ärmeren Schichten wiesen eine stärkere Gegenwartspräferenz auf als die reichen. Trifft dies zu, so wären die Reichen besser über die Zukunft informiert und würden demzufolge zukünftige Effekte von Budgetsalden stärker berücksichtigen als die ärmeren Bevölkerungsschichten. Nun sind es aber gerade die Schuldner und Sachvermögensbesitzer, also in der Mehrzahl die Reichen, die von einer Erhöhung der Inflations-

1) Vgl. Z.B. Schmah (1967), S. 233 ff., Walters (1967), S. 317, Gandenberger (1971), S. 386 und Hauser (1979), S. 85 
rate profitieren ${ }^{1)}$. Sie werden sich also trotzdem oder gerade weil sie besser über die Zukunft informiert sind, für Budgetdefizite aussprechen.

Eine weitere Bemerkung gilt der Problematik der Wirkungsverzögerung wirtschaftspolitischer Maßnahmen. Sind die neoinstitutionalistischen Annahmen bezüglich der Wirkungen von Budgetsalden auf Einkommen und Preisniveau korrekt, d.h. gilt die Wirkungskette

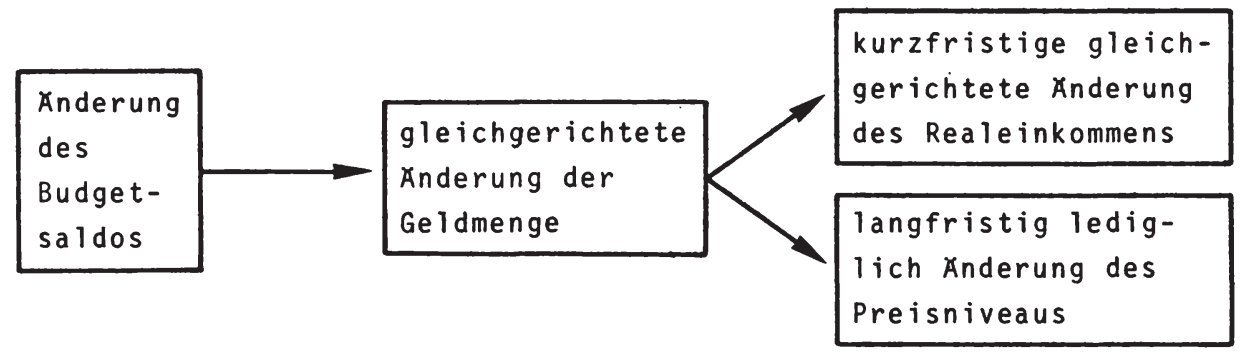

so läBt sich ihre These bezüglich der Informationsasymmetrien durch ein zusätzliches Argument stützen. Die Zeit, die verstreicht, bis eine Anderung der Geldmenge zu einer Anderung des Preisniveaus fuihrt, ist nämlich nicht in jeder situation gleich. Aufgrund dieser Variabilität der lags wissen die okonomen und erst recht die mit weniger ökonomischem Sachverstand ausgestatteten Wähler nicht exakt, wann der makroökonomische Effekt, der ihr Nutzenniveau beeinflussen wird, tatsächlich eintreten wird ${ }^{2}$. Diese Unsicherheit erhöht die Informationsprobleme über die Zukunft und spricht somit ebenso wie die vorige oberlegung für die von BW angenommenen Informationsasymmetrien ${ }^{3}$ ).

1) Vgl. Z.B. Folkers (1980), S. 279

2) Insbesondere Friedman hat auf die Variabilität der lags monetärer Impulse hingewiesen, vgl. Z.B. Friedman (1959), S. 87. Vgl. auch die Studie von Trapp (1976), insbesondere S. $143 \mathrm{f}$.

3) Für die gegenwärtigen Effekte von Budgetsalden spielen die genannten Wirkungsverzögerungen eine untergeordnete Rolle, da die gegenwärtigen Auswirkungen von Budgetsalden auf das Nutzenniveau nicht in erster Linie auf Veränderungen der Geldmenge zurückzuführen sind. So erhöhen Steuererleichterungen und Erhöhungen der Transferzahlungen ohne größere Verzögerungen das verfügbare Einkommen. 
1.4.5. Das Phänomen der Fiskalillusion

\subsubsection{Zum Begriff der Fiskalillusion}

Im Zusammenhang mit der Informationsdiskussion ist auch auf das Phänomen der Fiskalizzusion einzugehen, dem in der Argumentation von BW zentrale Bedeutung zukommt ${ }^{1}$ ).

Bereits RICARDO wird die These zugeschrieben, die Wirtschaftssubjekte unterlägen im Falle der Staatsverschuldung einer "public debt illusion", d.h. sie durchschauten nicht, daB die Staatsverschuldung ihnen zwar einen erhöhten Gegenwartskonsum ermögliche, aber dafür höhere Zukunftsbelastungen bringe ${ }^{2)}$. Weiterhin zeigte PUVIANI schon 1903 Möglichkeiten auf, das Belastungsgefühl der Zensiten durch unterschiedliche Besteuerungsinstitutionen zu beeinflussen ${ }^{3}$ ).

BUCHANAN ${ }^{4}$ ) und WAGNER ${ }^{5}$ ) haben diese Argumente ausgebaut und vor allem ihre allokativen Konsequenzen untersucht ${ }^{6}$ ). Das Phänomen der Fiskalillusion hat aber auch Konsequenzen für die Entscheidung eines Wählers für Defizite versus oberschüsse, die im folgenden untersucht werden.

Zunächst gilt es, das Phänomen der Fiskalillusion etwas näher zu beschreiben. Neuere Arbeiten über Fiskalillusion definieren sie als "the systematic misperception by individuals of the size of

1) Vgl. Z.B. Buchanan/Wagner (1977), S. $128 \mathrm{ff}$.

2) Vgl. Z.B. Gandenberger (1971), S. 383. Die Bezeichnung "public debt illusion" stammt von Vickrey (1961), S. 133.

3) Vgl. Puviani (1903), S. 39 ff. Vgl. hierzu auch Bartlett (1973), S. 92 ff. und die Untersuchungen von Schmölders (1959).

4) Vgl. Buchanan (1967), Kapitel 10

5) Vgl. Wagner (1976) und (1977a)

6) Vgl. hierzu Kapitel IV 
the burden of taxes and other public receipts borne by them and of the benefit returned for public expenditures"1). Zum besseren Verständnis der folgenden Ausführungen erscheint es nun zweckmäBig, die in dieser Definition enthaltenen Merkmale des Phänomens "Fiskalillusion" exakt herauszuarbeiten:

- Fiskalillusion bedeutet zunächst eine Falschwahrnehmung eines bestimmten Tatbestands.

- Die Falschwahrnehmung bezieht sich auf die Belastung mit Steuern und anderen öfentlichen Einnahmen und auf den Nutzen aus öffentlichen Ausgaben.

- Die Falschwahrnehmung ist systematisch, d.h. sie wirkt bei allen Individuen in eine Richtung ${ }^{2}$. Dabei nehmen $B W$ an, daB die. Individuen ihre an den Staat abzuführenden Steuerbeträge systematisch unterschätzen.

Das zweite Merkmal können wir zur Unterscheidung unterschiedlicher Formen der Fiskalillusion verwenden. So können wir die mit der Einnahmeinstitution "Staatsverschuldung" verbundene Illusion als "public debt illusion" oder "Schuldillusion" bezeichnen.

Gerade bei dieser Unterform der Fiskalillusion sehen einige Autoren einen Unterschied zum Phänomen der unvollkommenen Information. So unterscheidet CAVACO-SILVA ${ }^{3)}$ zwischen einer durch Unsicherheit bedingten falschen Schätzung eigener zukünftiger Steuerverbindlichkeiten und der eigentlichen Illusion, "the degree to which individuals do not perceive accurately the total of future taxes that a public borrowing contractually implies"4). Der entscheidende Unterschied zwischen einer durch unvollkommene Information bedingten

1) Pommerehne/Schneider (1978), S. 381

2) Vgl. Buchanan/Wagner (1977), S. 129

3) Vgl. Cavaco-Silva (1977), S. $34 \mathrm{ff}$. Vgl. auch seine Diskussion der von Vickrey (1961), S. 133, Buchanan (1964b), S. 150 f. und Ferguson (1964a), S. 227 verwendeten Definitionen.

4) Cavaco-Silva (1977), S. 37 
falschen oder unvollständigen Wahrnehmung und dem von RICARDO umschriebenen Phänomen der Illusion kommt jedoch auch in der Definition CAVACO-SILVAs nicht zum Ausdruck. Dieser Unterschied liegt in der Ursache der Falschwahrnehmung. Während sich die Falschwahrnehmung im ersten Fall durch das Rationalkalkuil eines informationssuchenden Individuums erklären läBt, sind im Falle der Illusion psychologische Effekte im Spiel, die nicht rational erklärt werden können ${ }^{1)}$. Andernfalls widerspräche die von RICARDO postulierte Schuldillusion seiner Annahme vollkommener Information. Korrekterweise müBte daher der Begriff "Illusion" für solche Phänomene reserviert werden, bei denen die Falschwahrnehmung durch nicht-ökonomische Effekte bedingt ist. Wenn wir in den folgenden Kapiteln trotzdem die obige Definition verwenden, so geschieht das deshalb, weil in neueren Arbeiten der Begriff Fiskalillusion vor allem Phänomene umschreibt, bei denen die falsche oder unvollkommene Wahrnehmung durch ökonomisch faBbare Effekte bedingt ist ${ }^{2}$. In diesem Fall sind dann in der Tat die mit einzelnen Einnahme- und Ausgabeinstitutionen verbundenen Informationskosten und -nutzen für das Auftreten von Fiskalillusion entscheidend. Die Determinanten der Informationskosten und -nutzen alternativer Finanzierungsinstrumente des Staates sollen nun im AnschluB erörtert werden.

\subsubsection{Der EinfluB alternativer Einnahmeinstitutionen auf die} Fiskalillusion

Die Literatur hat sich bisher vor allem mit der Analyse der Informationskosten der staatlichen Einnahmeinstrumente befaßt. Diese werden als von der "Sichtbarkeit" ${ }^{3)}$ oder "Merklichkeit"4) der fiskalischen Belastung ${ }^{5}$ ) abhängig angesehen.

1) "Es ist ein System, das uns weniger haushälterisch zu machen, uns für unsere tatsächliche Lage blind zu machen strebt." Ricardo (1923), S. 248

2) Vgl. Z.B. Pommerehne/Schneider (1980)

3) Vgl. Z.B. Bartlett (1973), S. 93 und den Oberblick bei Pommerehne/Schneider (1978), S. $384 \mathrm{f}$.

4) Vgl. Schmölders (1959)

5) Unter "Belastung" ist hier nur der an den Staat abgeführte Geldbetrag zu verstehen. Auf die mit alternativen Finanzierungsinstitutionen verbundene unterschiedliche Höhe des "excess burden" wird hier nicht eingegangen. Vgl. hierzu Z.B. Musgrave (1974), S. $119 \mathrm{ff}$. 
Diese "Sichtbarkeit" hängt insbesondere von folgenden institutionellen Bedingungen $a b^{1}$ ).

a) Vom Steuerobjekt. Während Steuern auf Einkommen und Vermögen von den Zensiten relativ stark wahrgenommen werden, sind Steuern, die an wirtschaftliche Transaktionen anknüpfen, weniger sichtbar. Am wenigsten sichtbar ist die Einnahmequelle "Staatsverschuldung", da ihr in der Gegenwart kein und in der Zukunft kein konkretes Steuerobjekt zugeordnet ist.

b) Von der Organisation des Besteuerungsverfahrens. Es macht einen Unterschied, ob ein und dieselbe Steuer, z.B. die Einkommensteuer, vom Arbeitgeber im Quellenabzugsverfahren einbehalten wird, oder ob die Zensiten zunächst ihr volles Bruttogehalt ausbezahlt und dann vom Staat eine "Rechnung" erhalten. Auch die zeitliche Verteilung der Steuerzahlung im Zusammenhang mit der Höhe des jeweils zu entrichtenden Steuerbetrags spielt eine Rol1e. Die Steuern werden eher bemerkt, wenn der Staat eine "Rechnung" schickt und wenn der gesamte Steuerbetrag auf einmal aufgebracht werden muß. Weiterhin kann die Steuer im Verkaufspreis eines Gutes enthalten sein, so daß der Konsument nicht zwischen dem Kostenanteil und dem Steueranteil am Gesamtpreis unterscheiden $k a n n^{2}$ ).

c) Von der Frage, ob es sich um eine alte oder eine neue Steuer hande1t. An eine bereits über längere Zeit erhobene Steuer haben sich die Zensiten bereits gewöhnt ${ }^{3}$ ). Eine neu eingeführte Steuer bzw. die Anderung einer bestehenden Steuer wird daher stärker wahrgenommen ${ }^{4}$ ).

1) Vgl. insbesondere die Systematik von Pommerehne/Schneider (1978), S. 384, aber auch Bartlett (1973), S. 93 ff. und Goetz (1977), S. $177 \mathrm{ff}$.

2) $\mathrm{Vgl}$. Goetz (1977), S. 178, der von einer "incorporation in product price"I1lusion spricht.

3) Diese These ist bereits seit langem als Canardsche Regel "Alte Steuern sind gute Steuern" (vgl. Schmölders (1970), S. 59 und (1975), S. 100) bekannt.

4) Vgl. insbesondere Buchanan (1967), Kapitel 5 sowie Schmölders (1959), S. 32 f. 
d) Von der Komplexität des staatlichen Einnahmesystems. Neben den bereits erwähnten Merkmalen einzelner Einnahmequellen spielt hierbei insbesondere die Zahl der Steuern und ihre Interdependenz eine Rolle ${ }^{1}$ ). Erhebt der Staat viele Steuern, so hat der Zensit gröBere Schwierigkeiten, seine tatsächliche Belastung zu ermitteln, als wenn der staat nur eine steuer erhebt.

Die genannten Sachverhalte erhöhen die Kosten der Information über die tatsächliche Belastung durch bestimmte Einnahmequellen und damit durch das gesamte Einnahmesystem. Hinzu kommt, daB der Grenznutzen der Information über die genaue Belastung insbesondere bei einigen kleinen Steuern minimal ist, so daB ein rationales Individuum keine vollkommene Information anstreben wird.

WAGNER weist indessen selbst darauf hin, daB aus den genannten Sachverhalten zwar auf eine Falschwahrnehmung, aber nicht unbedingt auf eine systematische Falschwahrnehmung, d.h. konkret auf eine Unterschätzung der Belastung geschlossen werden kann ${ }^{2}$ ). In der Tat führten empirische Untersuchungen zu uneinheitlichen Ergebnissen. So konnte WAGSTAFF ${ }^{3}$ ) zwar eine Falschwahrnehmung der fiskalischen Belastung infolge des Quellenabzugsverfahrens, aber keine systematische Unter- oder Oberschätzung der Belastung nachweisen. Auch die Untersuchungen von SCHMOLDERS ${ }^{4}$ ) lassen keinen SchluB auf eine systematische Falschwahrnehmung $z u$. WAGNER selbst weist in seiner Untersuchung zwar eine Unterschätzung aufgrund der durch die Zahl der Steuern gemessenen Komplexität des Steuersystems nach, doch vermag seine Untersuchung nicht zu überzeugen,

1) Vgl. insbesondere Wagner (1976)

2) Vgl. ebenda, S. 52. Pommerehne/Schneider (1978), S. 386, Fußnote 6 weisen sogar auf eine Reihe von Untersuchungen hin, die auf eine Oberschätzung der Belastung hindeuten.

3) Vgl. Wagstaff (1965). Die Untersuchungen Wagstaffs lassen eher auf eine Oberschätzung schließen.

4) Vgl. Schmb̈lders (1959) 
da sie nur vier Finanzierungsinstitutionen berücksichtigt und nur auf die Zahl der Steuern abstellt ${ }^{1}$.

1.4.5.3. Der EinfluB politischer Abstimmungsregeln auf die Fiskalillusion

POMMEREHNE/SCHNEIDER stelien bei ihrer auf dem Medianwählermode 1 1 2) basierenden Untersuchung der Fiskalillusion für den Bereich der 110 gröBten Schweizer Gemeinden zwar eine Unterschätzung der fiskalischen Belastung fest, bezeichnen den von ihnen ermittelten EinfluB der Fiskalillusion auf die öffentlichen Ausgaben jedoch als nicht überwältigend ${ }^{3)}$. Sie machen jedoch auf einen anderen Aspekt aufmerksam, der weniger auf die Kosten, sondern eher auf die Nutzen der Informationsgewinnung abhebt. Ihre Hypothese lautet ${ }^{4)}$ : Weit wichtiger als staatliche Einnahme- und Ausgabeinstitutionen sind die Abstimmungsregeln eines öffentlichen Gemeinwesens, da unterschiedliche Organisationsformen kollektiver Entscheidungsprozesse unterschiedliche Anreize für die Wähler auslösen, sich über die öffentliche Einnahme- und Ausgabegestaltung zu informieren. So haben wähler in repräsentativen Demokratien, in denen nur alle drei bis füf Jahre gewählt wird, nur eine geringe Kontrolle über das Ergebnis kollektiver Entscheidungsprozesse und können demzufolge aus einer besseren Information über ihre fiskalische Belastung nur wenig Nutzen ziehen. Dagegen haben die Wähler in direkten Demokratien eine direkte Kontrollmöglichkeit über öffentliche Einnahmen und Ausgaben, so daB die Anreize, sich über die fiskalische Belastung und die Art der öffentlichen Ausgaben zu informieren, viel größer sind.

1) Vgl. Wagner (1976), S. $55 \mathrm{ff}$. Er räumt selbst ein, daß in seiner Untersuchung "a revenue system in which all revenues are raised through a corporate income tax would be accorded the same index of simplicity as a revenue system in which all revenues are raised through a personal income tax". (Ebenda, S. 55)

2) Vgl. hierzu auch Pommerehne (1978)

3) Vgl. Pommerehne/Schneider (1978), S. 393

4) Vgl. ebenda, S. $394 \mathrm{f}$. 
Für den empirischen Test dieser Hypothese finden die beiden Autoren in der Schweiz ein nahezu ideales Untersuchungsgebiet vor. 27 der 110 gröBten Schweizer Gemeinden sind nämlich repräsentative Demokratien, 48 direkte Demokratien und 35 sind repräsentative Demokratien, in denen den Wählern die Möglichkeit des Referendums offensteht. Die konkret zu testende Hypothese lautet: "While in direct democracies there will be little or no evidence of fiscal burden illusion influencing public spending, in representative democracies the opposite will be true. In representative democracies with referenda, the influence of fiscal burden illusion is expected to be in-between ${ }^{1)}$."

Die Hypothese wird durch eine ökonometrische Untersuchung der Autoren in der Tendenz bestätigt. Lediglich in den 27 repräsentativen Demokratien ohne Referendum konnte ein statistisch signifikanter Einfluß der Fiskalillusion auf die öffentlichen Ausgaben festgestel1t werden. In den 48 direkten Demokratien war dagegen nur der EinfluB des Medianwählereinkommens, des Steuerpreises und der Bevölkerung statistisch signifikant ${ }^{2}$. Obertragen wir dieses für Schweizer Gemeinden ermittelte Ergebnis auf die Ebene des Zentralstaats, so sprechen vor allem zwei Gründe für das Auftreten von Fiskalillusion:

- westliche Zentralstaaten sind repräsentative Demokratien, so daß nur geringe Informationsanreize seitens der Wähler bestehen,

- westliche Zentralstaaten haben Zugang zu einem Finanzierungsinstrument, das den Schweizer Kommunen nur begrenzt zur Verfügung steht, und das wohl zu den am wenigsten merklichen Einnahmequellen des Staates zählt: der Kreditaufnahme ${ }^{3 \text { ). }}$

1) Pommerehne/Schneider (1978), S. 395. Weiterhin wird in repräsentativen Demokratien ein größeres Abweichen der Politiker von den Wünschen des Medianwählers postuliert. $\mathrm{Vgl}$. ebenda, S. $395 \mathrm{f}$.

2) Vgl. ebenda, Tabelle 2, S. 397

3) Empirisch ist die Frage, ob auf Zentralstaatsebene mit Fiskalillusion gerechnet werden muß, indessen noch nicht geklärt, zumal lediglich für Australien Untersuchungen vorliegen. Vgl. Pommerehne/Schneider (1980) und Pommerehne (1981) 
1.4.5.4. Zur Kritik BRUNNERs an der Annahme der Fiskalillusion

Die neoinstitutionalistische Prämisse der Fiskalillusion wurde vor allem von BRUNNER kritisiert ${ }^{1)}$. Seiner Ansicht nach ist die Annahme einer Wahrnehmungsdiskrepanz, die sich gleichmäßig auf alle Wähler sowie auf alle staatlichen Einnahme- und Ausgabeprogramme erstreckt, nicht haltbar. Er ersetzt diese Prämisse durch die Annahme einer Asymmetrie in der Verteizung der Informationskosten über die Kosten und Nutzen alternativer wirtschaftiicher Maßnahmen, die mit der Asymmetrie der Verteilung der Kosten und Nutzen dieser Maßnahmen einhergeht ${ }^{2)}$. Kennzeichnend für die Argumentation BRUNNERs ist die Unterscheidung allgemeiner und spezifischer Einnahme- und Ausgabeprogramme. Spezifische Programme sind dadurch charakterisiert, daß sie speziellen Bevölkerungsgruppen zugute kommen. Die Kosten und Nutzen spezifischer Programme sind daher ungleichmäßiger verteilt als die Kosten und Nutzen allgemeiner Programme. Anstelle der Annahme der Fiskalillusion verwendet BRUNNER die folgenden Prämissen ${ }^{3)}$ :

- "Information costs about costs and benefits of general programs are Zarge relative to benefits."

- "Information costs about costs and benefits of specific programs are relatively smazz to 'positively affected group' and comparatively Zarge to 'negatively affected group'."

Beide Prämissen treffen jedoch m.E. nicht den Kern des Informationsproblems, dem sich der Wähler gegenübersieht, bzw. sie bedürfen einer näheren Erklärung. Die Wahrnehmung der Kosten und Nutzen eines Programmes hängen nicht davon ab, ob sie sich positiv oder negativ auf das Nutzenniveau auswirken, sondern davon, wie der einzelne durch das Programm direkt betroffen wird ${ }^{4}$. Dabei spielt

1) Vgl. Brunner (1978), S. 672

2) Vgl. ebenda und derselbe (1975), S. 852

3) Brunner (1975), S. 852 und (1978), S. 672

4) Vgl. Beetz (1978), S. $51 \mathrm{f}$. 
die GröBenordnung des Programms eine Rolle ${ }^{1)}$. Gehen wir von einem Programm aus, das eine gegebene Einnahme- bzw. Ausgabesumme einerseits auf alle Wähler, andererseits auf eine spezielle Gruppe verteilt, so entfällt im ersten Fall eines allgemeinen Programms auf den einzelnen nur ein geringer Teil, der weniger merklich ist als im zweiten Fall, in dem dieselbe Summe auf eine geringere Anzahl von Wählern verteilt wird. Hieraus können wir schlieBen, daB - ein Ausgabe-(Einnahme-) Programm gegebener Höhe vorausgesetzt - spezifische Programme stärker wahrgenommen werden als allgemeine. Wird demnach Z.B. ein spezifisches Ausgabeprogramm ${ }^{2}$ ) bestimmter Höhe beschlossen und durch alzgemeine steuern finanziert, so sind die Informationskosten der Begünstigten über den Nutzen des Programms geringer als die Informationskosten der benachteiligten Steuerzahler über die exakte Höhe der Belastung. AuBerdem wird der Nutzen der Information über die exakte Belastung für die Benachteiligten niedrig sein, da diese nur eine geringe GröBenordnung hat ${ }^{3}$ ). Daher wird die durch das spezifische Ausgabeprogramm begünstigte Gruppe den Nutzen des Programms stärker wahrnehmen als die benachteiligte Gruppe die Kosten der Finanzierung des Programms.

Nehmen wir nun an, daB nicht nur ein spezifisches Ausgabeprogramm beschlossen wird, sondern mehrere, und daB alle Ausgaben durch allgemeine Steuern finanziert werden, so bedeutet das, daB alle Begünstigten den Nutzen der Ausgaben stark, alle Benachteiligten die Kosten aber nur in geringem AusmaB wahrnehmen. Im Extremfall, in dem alle Staatsbürger durch irgendein allgemein finanziertes Programm speziell begünstigt werden, sind aber Begünstigte und Be-

1) Gemeint ist nicht die Größenordnung des Programms an sich, sondern die Höhe der auf den einzelnen entfallenden Einnahme- bzW. Ausgabesumme. $\mathrm{Vgl}$. auch Frey/Garbers (1972), die in anderem Zusammenhang darauf hinweisen, da $B$ bestimmte Reizschwellen überschritten werden müssen, bevor es zu einer Wahrnehmung kommt.

2) Alternativ wäre an eine spezielle Gruppen begünstigende Steuersenkung zu denken.

3) In der Tat scheint Brunner bei Formulierung seiner Prämissen ein spezielles Ausgabeprogramm vor Augen $z u$ haben, das durch allgemeine Steuern finanziert wird. In diesem Fall sind nämlich die Informationskosten der benachteiligten Allgeme inheit hoch, der Nutzen der Information ist niedrig. 
nachteiligte ein und dieselben Personen. Diese nehmen den Nutzen der Programme stärker wahr als die Kosten, d.h. sie unterliegen der Fiskalillusion. Sind Begünstigte und Benachteiligte nicht völlig identisch, so tritt die Fiskalillusion in abgeschwächter Form auf. Werden daher die Annahmen BRUNNERs über das Informationsmuster der Wähler in der oben beschriebenen Art und Weise abgeändert bzw. präzisiert, so sprechen diese Annahmen nicht gegen, sondern für die Prämisse der Fiskalillusion.

\subsubsection{Die Auswirkungen der Fiskalillusion auf die Entscheidung} der Wähler für Defizite versus Oberschüsse

Welche Bedeutung kommt nun dem Illusionsphänomen bei der Entscheidung eines Wählers für Defizite versus überschüsse zu? Im Falle der Schuldillusion im eigentlichen Sinne liegen die Konsequenzen klar auf der Hand. Die Tendenz zu Defiziten verstärkt sich in dem Maße, wie die mit den Defiziten verbundenen zukïnftigen Zins- und Tilgungsverbindlichkeiten von den wählern nicht wahrgenommen werden, da die Wähler davon ausgehen, einen Teil der in der Gegenwart empfangenen Realeinkommens- oder Konsumerhöhungen umsonst zu bekommen ${ }^{1)}$. Mit einer derartigen Illusion ist insbesondere zu rechnen, wenn Budgetdefizite durch Verschuldung bei der Zentralbank, also durch Geldschöpfung, finanziert werden, da in diesem Fall keine Zins- und Tilgungsverbindlichkeiten entstehen. Wohl kann aufgrund des Defizits die Inflationsrate steigen, und diese Erhöhung der Inflationsrate kommt einer Besteuerung der Bargeldhaltung

1) Diese Folgerung gilt auch für den Fall, daß die Individuen die für die Gesel1schaft in der Zukunft anfallenden Verbindlichkeiten klar erkennen, aber aus bestimmten Gründen davon ausgehen können, daß sie persönlich nicht zur Bezahlung der Verbindlichkeiten herangezogen werden. $\mathrm{Vgl}$. hierzu die Ausführungen über Verteilungseffekte in III.1.4.9. 
gleich, jedoch wird diese äuBerst indirekte "Art der Besteuerung" von den Wählern nur in sehr geringem Ausmaß wahrgenommen ${ }^{1}$ ).

In ähnlicher Weise senkt auch die Unterschätzung gegenwärtiger Steuerzahlungen den wahrgenommenen "Preis" öffentlicher Guiter. Diese Preissenkung hat allerdings keinen direkten EinfluB auf die Entscheidung des Wählers für Defizite versus Oberschuisse. Sie kann die Tendenz zu Defiziten jedoch mittelbar verstärken. Fordern nämlich die Wähler von den Politikern aufgrund der Preissenkung mehr öffentliche Güter und wollen die Politiker dieser Forderung entsprechen, so müssen die zusätzlichen öffentlichen Güter finanziert werden. Sind Steuererhöhungen nicht oder nur in geringem Maße durchzusetzen, so verstärkt sich die Tendenz zu Defiziten ${ }^{2}$ ).

Als Fazit bleibt somit festzuhalten:

Die These von BW, in demokratischen Staten bestehe eine Tendenz zu Budgetdefiziten, trifft zu, wenn die von den Autoren unterstellten makroökonomischen Effekte von Budgetsalden realistisch sind, wenn die Anti-Inflationsmentalität der Bevölkerung nicht sehr ausgeprägt ist, und wenn neben den von ihnen berücksichtigten Effekten keine weiteren auftreten, die das Nutzenniveau des Medianwählers vermindern.

In den folgenden Abschnitten sollen nun alternative Effekte von Budgetsalden auf Einkommen und Preisniveau sowie weitere, von BW nicht berücksichtigte Effekte auf das Nutzenniveau der Wähler untersucht werden.

1) "In terms of the fiscal perceptions of citizens, however, inflation does not seem at all equivalent to a tax . . . Individual citizens are likely to be less informed about the probable costs of an 'inflation tax' than they are about even the most indirect and complex explicit levy." $\mathrm{Vgl}$. Buchanan/Wagner (1977), S. 142. Abgesehen von dieser "tax on cash" findet eine teilweise Finanzierung des Defizits über die progressive Einkommensteuer statt, wenn die Einkommen aufgrund der Inflation nominal steigen. Auch diese erhöhten zukünftigen Steuerverbindl ichkeiten werden von den Wirtschaftssubjekten unterschätzt.

2) Vgl. auch Greene (1973), S. 215 
1.4.6. Alternative Wirkungen von Budgetsalden auf Realeinkommen und Inflationsrate

OLSON ${ }^{1}$ ) hat darauf hingewiesen, daB richtig dosierte Budgetuberschüse in einer Phase durch obernachfrage bedingter Inflation keineswegs zu Nutzenverlusten bei den Wählern führen müssen. Gelingt es, marginale Budgetüberschüsse so zu bemessen, daB sie lediglich die monetäre Obernachfrage abschöpfen, so werden damit das Realeinkommen und der Realkonsum nicht tangiert: ". . an antiinflationary policy need involve no 'reduction in presently enjoyed consumption' as compared with an inflationary fiscal policy")." Die für die Entscheidung des Medianwählers für Defizite versus oberschüsse relevanten. Differentialquotienten haben dann die folgenden vorzeichen:

(III.8.2) $\frac{\partial\left(y_{0}^{M} / p_{0}\right)}{\partial B}=0$,

wobe $i$

(III.9.2) $\quad \frac{\partial y_{0}^{M}}{\partial B}<0$

und

(III.10.2) $\quad \frac{\partial p_{0}}{\partial B}<0$.

AuBerdem gilt

(III.11.2) $\frac{\partial x_{0}^{M}}{\partial B}=0$.

1) $\mathrm{Vgl} .01 \mathrm{son}(1978)$, S. 109

2) Ebenda 
Akzeptieren wir den Einwand OLSONs, so ist eine Ablehnung von Budgetüberschuissen bzw. eine Forderung von Budgetdefiziten in Zeiten durch obernachfrage bedingter hoher Inflationsraten nur dann zu erwarten, wenn der Medianwähler zu den Inflationsgewinnern zählt, oder wenn er glaubt, er werde von den zur Bekämpfung der Inflation erforderlichen kontraktiven Maßnahmen stärker negativ betroffen als er durch die Vermeidung der Inflation profitiert.

Die Argumentation OLSONs vermag allerdings weniger zu überzeugen als die von BW postulierte Alternative. Zunächst ist darauf hinzuweisen, daB Inflation auch in einer nahezu vollbeschäftigten Wirtschaft nicht allein die Folge einer globalen obernachfrage ist. Weiterhin unterstellt OLSON implizit, daB das Preisniveau annähernd so schnell auf fiskalpolitische Maßnahmen reagiert wie das Nominaleinkommen und der Konsum. Ein marginaler BudgetüberschuB hat aber, bevor er sich über Nachfrage- oder Geldmengenänderungen auf das Preisniveau auswirkt, die von BW aufgezeigten Effekte. Zunächst ändern sich das verfügbare Nominaleinkommen und der Konsum öffentlicher Güter und erst aufgrund der Reaktionen der von der MaBnahme betroffenen Wirtschaftssubjekte und der Zentralbank kommt es zu Nachfrage- und Geldmengeneffekten, die wiederum mit zeitlicher Verzögerung zu Preisniveauänderungen führen ${ }^{1)}$. Die zeitliche Abfolge des Wirkungsmechanismus fiskaipolitischer Maßnahmen spricht somit eher für die Argumentation der Neoinstitutionalisten.

\subsubsection{Unterschiedliche Zeitpräferenzen}

Unterschiedliche Zeitpräferenzen beeinflussen das Nutzenniveau eines Wählers nicht nur über sein optimales Informationsniveau ${ }^{2}$, sondern können auch einen direkten EinfluB auf das Nutzenniveau ausüben. Dieser Effekt ergibt sich aus der Tatsache, daB ärmeren Bevölkerungsschichten die Möglichkeit verschlossen bleibt, sich

1) Diese Wirkungskette gilt zumindest für den Bereich der Bundesrepublik Deutschland, wo eine Verschuldung bei der Zentralbank praktisch ausgeschlossen ist (vgl. § 20 BBankG).

2) Vgl. III.1.4.4. 
zu dem relativ günstigen Kapitalmarktzinssatz zu verschulden ${ }^{1)}$. Diesen ärmeren Bevölkerungsschichten wird durch die Staatsverschuldung ein höherer Gegenwartskonsum und damit möglicherweise die Erreichung eines höheren Nutzenniveaus ermöglicht.

Wir wollen uns diesen Sachverhalt anhand eines Zwei-Perioden-Modells verdeutlichen ${ }^{2}$ : Abbildung 3 zeigt die Bilanzgerade CPD eines Individuums, das zum Zeitpunkt $t_{0}$ einen Kredit aufnehmen möchte, dem dies aber nur zu einem höheren als dem für staatliche Wertpapiere geltenden Zinssatz möglich ist ${ }^{3)}$. Links von p entspricht die Steigung dem Zinssatz für Staatspapiere auf dem Kapitalmarkt $r_{K}$, rechts von $P$ verläuft die Bilanzgerade aufgrund des höheren Sollzinssatzes steiler.

Durch ein statliches Budgetdefizit wird die Situation des Individuums verändert ${ }^{4}$ ). Einerseits erhält es im Zeitpunkt $t_{0}$ ein höheres verfügbares Einkommen. Andererseits muB das Defizit im Zeitpunkt $t_{1}$ verzinst und getilgt werden, wodurch sich das Zukunftseinkommen vermindert. Nehmen wir, um Umverteilungseffekte bei der Verzinsung und Tilgung auszuschlieBen ${ }^{5)}$ an, die Zins- und Tilgungsverbindlichkeiten würden durch eine verteilungsneutrale Pauschal-

1) Vgl. Walters (1967), S. 317

2) Die Analyse ist angelehnt an Gandenberger (1971), S. 384 f. Im Unterschied zu Gandenberger interessieren an dieser Stelle jedoch nicht die Differentialeffekte von Steuer- und Anleihefinanzierung, sondern die spezifische Wirkung eines Defizits auf das durch Gegenwarts - und Zukunftskonsum bestimmte Nutzenniveau eines Individuums. Vgl. auch Gandenberger (1980a), S. 492

3) Da wir den Einfluß der Zeitpräferenzen auf das optimale Informationsniveau bereits diskutiert haben, können wir zur Untersuchung der jetzt interessierenden Effekte vollkommene Information annehmen. Des weiteren vernachlässigen wir etwaige Effekte der Staatsverschuldung auf das Zinsniveau auf dem Kapitalmarkt. Vgi. hierzu III.1.4.4. und III.1.4.9.

4) Wir gehen im folgenden von einer Steuersenkung bei konstanten Staatsausgaben aus. Diese Annahme wird getroffen, da defizitfinanzierte öffentliche Güter vom Individuum wohl nur in Ausnahmefällen als Substitut für private Güter angesehen werden.

5) Vgl. hierzu III.1.4.9. 


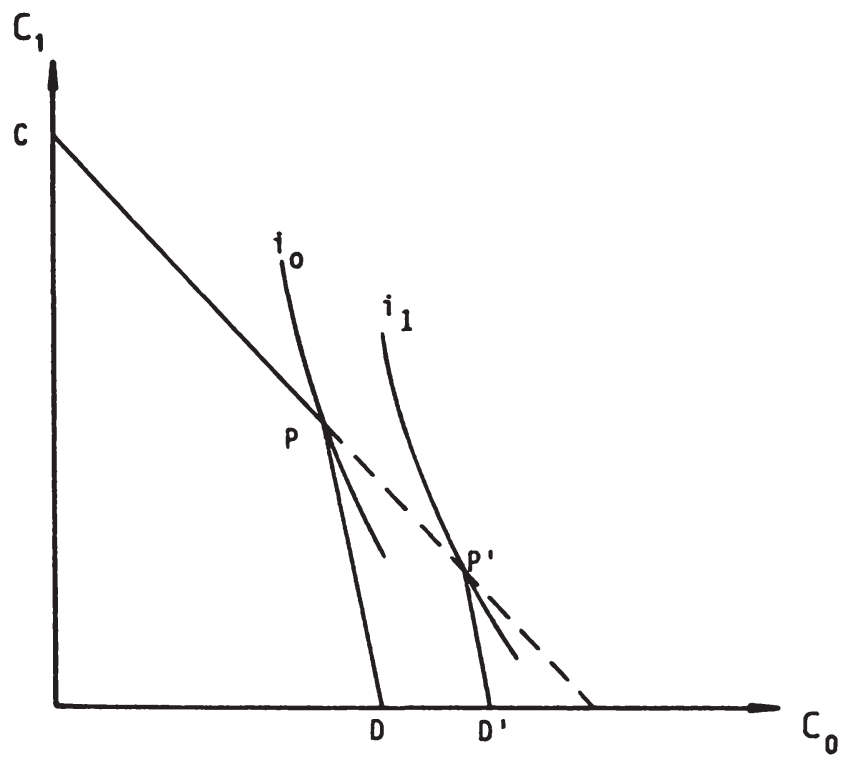

Abbildung 3: Wirkungen der Staatsverschuldung auf das Nutzenniveau eines Individuums mit Gegenwartspräferenz ${ }^{1}$ )

steuer aufgebracht, so repräsentiert CP'D' die neue Bilanzgerade des Individuums. Die Situation ist also vergleichbar mit der Gewährung eines Staatskredits zu Kapitalmarktkonditionen. Durch Budgetdefizite wird somit das Nutzenniveau derjenigen Individuen, die zum Kapitalmarktzinssatz leihen wollen und dies bisher nicht konnten, erhöht ( $i m$ dargestellten fall von $i_{0}$ auf $i_{1}$ ). Individuen mit einer geringeren Gegenwartspräferenz können wie bisher ihre in der Gegenwart nicht zum Konsum benötigten Einkommensteile auf dem Kapitalmarkt anlegen. Ihre Situation wird durch die staatliche Kreditaufnahme c.p. nicht verändert.

1) Vgl. Gandenberger (1971), S. 385 


\subsubsection{Crowding-out-Effekte der Staatsverschuldung}

Von "Crowding-out-Effekten" sprechen wir, wenn private Ausgaben durch staatliche Ausgaben ganz oder teilweise verdrängt werden ${ }^{1)}$. Diese Verdrängung ist bei konstanten Preisen vor allem durch eine Erhöhung des Zinssatzes aufgrund staatiicher Budgetdefizite bedingt, welche die als zinselastisch angenommenen privaten Investitionen vermindert. Weiterhin kann es aufgrund der defizitfinanzierten staatlichen Nachfrage in einigen Sektoren zu Preissteigerungen kommen, so daB die private Nachfrage in diesen Sektoren zurückgedrängt wird ${ }^{2}$. Letztendlich führt ein statliches deficit-spending daher nur zu einer geringfügigen - im Grenzfall zu überhaupt keiner - Erhöhung des Volkseinkommens und der Beschäftigung, da die staatlichen Ausgaben lediglich an die Stelle der privaten Ausgaben treten und langfristig keine zusätzliche Nachfrage bewirken ${ }^{3}$.

In der Literatur werden in diesem Zusammenhang vor allem zwei Effekte diskutiert, die zu Nutzenminderungen führen können. Zum einen sinkt, wenn nicht ein Großteil der Krediteinnahmen des Staates für investive Zwecke ausgegeben wird, der zukünftige Kapitalstock und damit das zukünftige Realeinkommen ${ }^{4}$ ). Zum anderen erleiden die durch die statliche Kreditaufnahme verdrängten privaten Kreditnehmer eine Nutzenminderung ${ }^{5}$. Beide Effekte werden von BW gesehen. Die Wirkung auf den zukünftigen Kapitalstock ${ }^{6}$ ) wird allerdings

1) Für diese Phänomene haben sich die Begriffe "vollständiges" und "partielles" crowding-out eingebürgert. $\mathrm{Vgl}$. Z.B. Rose (1980), S. $357 \mathrm{f}$.

2) Vgl. Brunner (1970), S. $21 \mathrm{f} . \mathrm{Vgl}$. zur Diskussion von anderen Crowding-outEffekten vor allem Carlson/Spencer (1975). Eine exaktere Diskussion über das Auftreten von Crowding-out-Effekten bleibt Kapitel V.2.1. vorbehalten. An dieser Stelle wird nur diskutiert, wie sich Crowding-out-Effekte im Falle ihres Auftretens auf das Nutzenniveau der Wähler auswirken können.

3) Vgl. auch Brunner/Meltzer (1976), die sogar eine negative Multiplikatorwirkung postulieren.

4) In diesem Effekt sehen die Vertreter des "Aggregate-Investment-Ansatzes" zur intertemporalen Lastverteilung eine "Last", die zukünftige Generationen trifft. Vgl. z.B. Modigliani (1964), S. 109

5) Vgl. Z.B. Gandenberger (1972), S. 201 und Tobin (1965), S. 680

6) Vgl. Buchanan/Wagner (1977), S. $66 \mathrm{ff}$. 
nicht weiterverfolgt, was wohl durch die Annahme eines geringen Informationsniveaus über die Zukunft zu erklären ist. Dagegen werden die Wirkungen auf den verdrängten Kreditnehmer in den Bereich der "secondary repercussions"1) verwiesen.

Sowohl die Einstufung der Wirkungen auf die verdrängten Kreditnehmer, die aller Wahrscheinlichkeit nach nicht wahlentscheidend sein werden ${ }^{2)}$ als auch die Vernachlässigung der Effekte auf den zukünftigen Kapitalstock, erscheinen jedoch gerechtfertigt. Im Gegensatz zu Inflationswirkungen, die in einem noch überschaubaren Zeitraum auftreten werden, beeinträchtigt ein geringerer Kapitalstock die ökonomische Position eines Individuums erst in ferner Zukunft. Diese Wirkungen werden daher, selbst wenn sie wahrgenommen werden, stark abdiskontiert. Des weiteren düfte den Individuen das Problem der Inflation, mit dem sie in der gegenwärtigen Situation fast täglich konfrontiert werden, stärker bewuBt sein als die Probleme, die mit einem verminderten Kapitalstock in der Zukunft verbunden sind. Daher werden die Individuen diese Wirkungen nur in geringem Umfang wahrnehmen.

\subsubsection{Verteilungswirkungen}

Verteilungswirkungen können sich zunächst aufgrund einer durch die staatliche Kreditnachfrage bedingten Erhöhung der Zinseinkommen ergeben ${ }^{3)}$. Fragt der Staat kredite nach, so kommt es zu einem erhöhten Kapitalmarktzinsniveau ${ }^{4}$, das die Besitzer von langfristigen Wertpapieren begünstigt. Die Zinssteigerung bewirkt aber gleichzeitig eine Senkung der Wertpapierkurse, also eine Abnahme des pri-

1) Vgl. Buchanan (1958), S. 93 und (1962), S. 72

2) Weiterhin ist fraglich, ob den Kreditnehmern die Ursache ihrer Verdrängung uberhaupt bewußt wird.

3) Vgl. Gandenberger (1970a) und (1974), aber auch die portfoliotheoretische Erweiterung des Ansatzes durch Dieckheuer (1979).

4) Mit einer Zinssteigerung ist vor allem in der Hochkonjunktur zu rechnen. $\mathrm{Vgl.}$ Gandenberger (1980a), S. 484 f. 
vaten Nettovermögens in der Volkswirtschaft ${ }^{1}$, und damit c.p. einen Nutzenverlust. Eine Netto-Staatsschuldentilgung hat die umgekehrten Effekte.

Die genannten Wirkungen dürften jedoch für die Entscheidung eines Individuums für Defizite versus Oberschüsse nur in Ausnahmefällen von Bedeutung sein. Dies gilt insbesondere in Anbetracht der Feststellung, daß sich während der letzten Jahre $70-80 \%$ aller Staatsschuldtitel im Besitz des privaten Bankensystems befanden ${ }^{2}$. Es ist zwar möglich, daB aufgrund dieser Tatsache auch die Vielzahl der Sparer mit kleineren und mittleren Einkommen indirekt an den Zinserträgen aus Staatsschulden partizipiert ${ }^{3)}$. Doch selbst wenn die Sparer diesen Zusammenhang erkennen sollten, ist es für sie nahezu unmöglich, den Effekt der Staatsverschuldung auf ihr Zinseinkommen einigermaßen exakt zu ermitteln.

Weitere Verteilungseffekte können sich bei einer Erhöhung der Staatsverschuldung aufgrund der gesamtwirtschaftichen Zusammenhänge ergeben. Sie sind durch das jeweils unterstellte volkswirtschaftliche Verteilungsmodell bedingt. Im Zustand der Vollbeschäftigung resultiert jedoch stets ein Effekt zugunsten der Bezieher von Gewinn-bzw. Kapitaleinkommen, und zwar unabhängig davon, ob z.B. neoklassische oder kaldorianische Verteilungsvorstellungen zugrundegelegt werden. Während ein Budgetdefizit unter neoklassischen Annahmen c.p. zu einem Rückgang des privaten Kapitalstocks und damit zu einer Steigerung der Kapitalrendite führt ${ }^{4}$, führen Defizite im kreislaufanalytischen Modell nach KALDOR zu einer Erhöhung der Gewinnquote infolge von Preisniveausteigerungen und damit auch zu einer Erhöhung des Anteils der Gewinnbezieher am ge-

1) Gandenberger (1980a), S. 489 spricht von einer. "Baisse festverzinslicher Vermögenswerte".

2) Vgl. 2.B. Cassel (1980), S. 274

3) Vgl. Willms (1978), S. 444. Vgl. aber auch Wissenschaftlicher Beirat (1979), S. $65 \mathrm{ff}$., wo auf die negativen Verteilungswirkungen des hohen Anteils der Banken am Bestand der Staatspapiere hingewiesen wird.

4) Vgl. Folkers (1981), S. 166 
samtwirtschaftlichen Vermögensbestand ${ }^{1)}$. In der Hochkonjunktur dürten sich aufgrund dieses Effekts nur die Bezieher von Gewinnbzw. Kapitaleinkommen für ein marginales Budgetdefizit aussprechen, während die Bezieher von Arbeitseinkommen entgegen der Ansicht von BW Budgetüberschüsse fordern müßten. Vollzieht sich jedoch die Erhöhung der Gewinne wie im KALDOR-Modell über Preissteigerungen bei konstantem Nominallohn, so sind dieser Betrachtung dieselben Einwände entgegenzuhalten wie der Argumentation OLSONs. Defizite erhöhen zunächst das Realeinkommen bzw. den Realkonsum, ehe sie zeitverzögert zu den von den Individuen negativ bewerteten Preissteigerungen führen. Auch die Aussagen des neoklassischen Modells gelten nur für das langfristige Gleichgewicht, so daB kurzfristig durchaus nicht mit einer Ablehnung von Budgetdefiziten durch die Bezieher von Arbeitseinkommen gerechnet werden muß.

Werden Defizite nicht - wie beispielsweise in III.1.4.7. angenommen - durch eine Pauschalsteuer verzinst und getilgt, sondern über das progressiv wirkende allgemeine Steuersystem, so ergeben sich Verteilungswirkungen zugunsten der ärmeren Bevölkerungsschichten. Diese Verteilungseffekte können kombiniert mit den bereits diskutierten Effekten der Zeitpräferenz auftreten. Abbildung 3 auf Seite 45 läßt sich dann wie folgt modifizieren.

1) Vgl. Folkers (1981), S. 235 


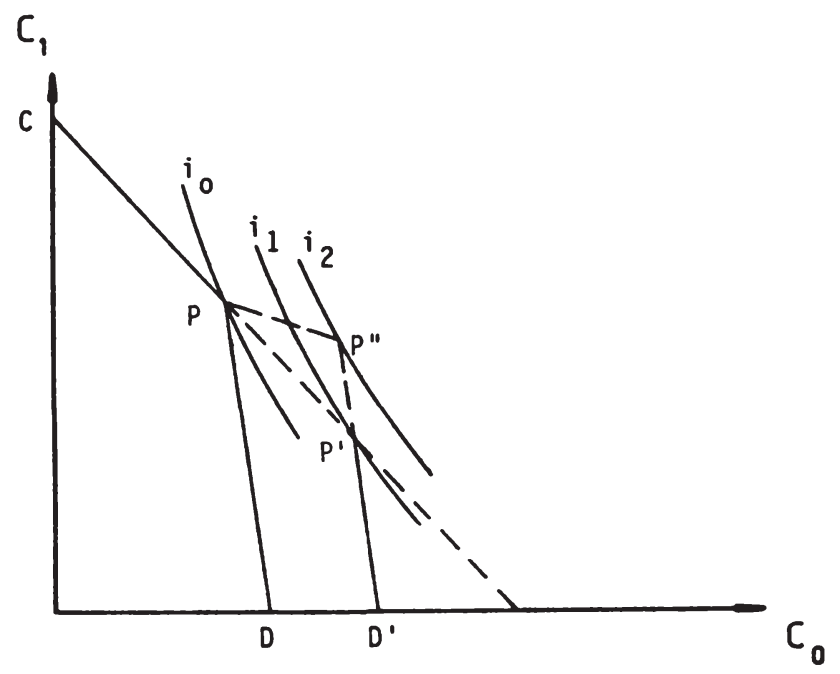

Abbildung 4: Wirkungen der Staatsverschuldung auf ein unterproportional besteuertes Individuum mit Gegenwartspräferenz

Da die Reicheren einen Teil der bei der Pauschalsteuer auf die Armen entfallenden Zins - und Tilgungsverbindlichkeiten mitfinanzieren, wird der Kredit für das von uns betrachtete Individuum billiger, d.h. die Budgetlinie wird rechts von $P$ flacher, so daB das Individuum das höhere Nutzenniveau $i_{2}$ erreicht.

Werden Defizite gar teilweise durch neuerliche staatliche Kreditaufnahme verzinst und getilgt oder können die Individuen damit rechnen, daB sie aufgrund der Erreichung des Rentenalters oder eines Umzugs ins Ausland ${ }^{1}$ nicht voll für die Zins- und Tilgungsverbindlichkeiten aufkommen müssen, so "verbilligt" sich ihr Gegenwartskonsum zusätzlich in dem MaBe, wie sie die Steuerverbindlichkeiten ihrer Nachkommen nicht als ihre eigenen ansehen ${ }^{2)}$. Ihr Votum für Defizite liegt dann auf der Hand.

1) Das Umzugsargument spielt wohl keine entscheidende Rolle. Es gewinnt jedoch an Bedeutung, wenn wir die Verschuldung einer dezentralen Gebietskörperschaft, z.B. eines Bundeslands oder einer Gemeinde, betrachten. Vgl. Z.B. Greene (1973), S. $211 \mathrm{ff}$.

2) Vgl. Buchanan/Wagner (1977), S. 140 f., aber auch Gandenberger (1971), S. 388 und $(1980 \mathrm{a})$, S. 490 und S. 493. 
1.4.10. Psychologische Grenzen der Staatsverschuldung

Bisher haben wir angenommen, daß die Wähler ihre Entscheidung für Defizite oder Oberschüsse von deren Wirkungen auf GröBen wie Einkommen, Konsum, Preisniveau etc. abhängig machen. Neben ökonomischen Effekten können jedoch auch psychologische Abneigungen der Wähler gegenüber der Staatsschuld eine Rolle spielen. SCHLESINGER geht jedenfalls davon aus, "daß eine gewisse Skepsis gegenüber der Staatsverschuldung zu einem festen Bestandteil der menschlichen Psyche geworden ist" 1 ). In der Bundesrepublik spielen in diesem Zusammenhang neben der Feststellung, daB jegliche Schuldenaufnahme in weiten Kreisen der Bevölkerung noch als etwas Unsolides angesehen wird ${ }^{2}$, oder $d a B$ eine steigende Verschuldungsquote als Ausdruck eines wachsenden Einflusses des Staates auf die Privatwirtschaft interpretiert und deshalb vor allem von konservativen Wählern abgelehnt wird ${ }^{3}$ ), in erster Linie die Erfahrungen nach dem Ersten Weltkrieg eine Rolle, als die Statsanleihen durch eine Hyperinflation entwertet wurden ${ }^{4}$ ).

Derartige psychologische Effekte können durchaus ein starkes Gewicht haben und zu einer Ablehnung von Budgetdefiziten durch die Wähler führen. Die mit staatlichen Defiziten verbundene Inflationsangst der Bevölkerung düfte indessen empirisch kaum feststellbar sein ${ }^{5)}$, was eine Bewertung des Psychologie-Arguments erschwert.

1) Schlesinger (1975), S. 1080

2) Schmölders (1975), S. 43

3) Vgl. auch Neumark (1949), S. 536 f., der auf die Gefahr hinweist, daB sich der Staat durch übermäßige Staatsverschuldung "offen zum Leviathan umgestaltet" (S. 537).

4) Vgl. Sachverständigenrat (1967), Tz, 518 sowie Ziffzer (1980), S. 500

5) Feststellen läßt sich unter bestimmten Bedingungen lediglich die Bewertung der Inflation an sich (vgl. hierzu Abschnitt III.2.), nicht jedoch die Bewertung des Zusammenhangs zwischen Staatsverschuldung und Inflation. 


\subsubsection{Fazit der theoretischen Argumente}

Die theoretische Analyse der Effekte staatlicher Budgetsalden, insbesondere staatlicher Budgetdefizite, hat gezeigt, daB ein und derselbe Personenkreis von staatlichen Budgetdefiziten sowohl profitieren als auch Nutzenverluste erleiden kann ${ }^{1)}$. Somit ergibt sich nicht nur das Problem zu ermitteln, welcher Bevölkerungsschicht der Medianwähler angehört ${ }^{2}$, es erhebt sich auch die Frage nach der relativen Stärke der theoretisch begründbaren Effekte. Der Versuch, die relative Stärke der genannten Effekte empirisch zu ermitteln, erscheint daher notwendig und lohnend.

\section{Versuch einer Oberprüfung der BUCHANAN/WAGNER-These anhand po-} litometrischer Schätzungen

Die Oberprüfung der BW-These erfordert empirische Untersuchungen daruber, wie sich Defizite und Oberschüsse auf das Abstimmungsverhalten der Wähler auswirken. Derartige Untersuchungen liegen zum gegenwärtigen Zeitpunkt noch nicht vor bzw. sind erst in Ansätzen vorhanden ${ }^{3}$. Dagegen exisitert eine Vielzahl empirischer Arbeiten, die den statistischen Zusammenhang der Variablen Arbeitslosenquote, Inflationsrate und Wachstum des verfügbaren Realeinkommens mit dem Abstimmungsverhalten der Wähler untersuchen. Diese Untersuchungen können zur oberprüfung der BW-These herangezogen werden, wenn wir die von den beiden Autoren postulierten Wirkungen der Budgetsalden auf diese makroökonomischen Variablen akzeptieren ${ }^{4}$.

1) So können "die Armen" einerseits durch die Umverteilungseffekte der Staatsverschuldung profitieren, andererseits zählen sie zu den Inflationsverlierern.

2) Brunner (1978), S. 666 weist darauf hin, daß weder die Politiker noch die Wähler selbst genau wissen, wer zur Gruppe der Medianwähler gehört. Auch Brunner geht jedoch davon aus, daß der Medianwähler in den unteren Einkommensklassen angesiedelt ist.

3) Vgl. Pommerehne (1981)

4) Vgl. aber die empirische Untersuchung des Zusammenhangs zwischen Budgetsalden und Inflation in Kapitel $\mathrm{V}$. 
Der ProzeB läBt sich schematisch wie folgt verdeutlichen:

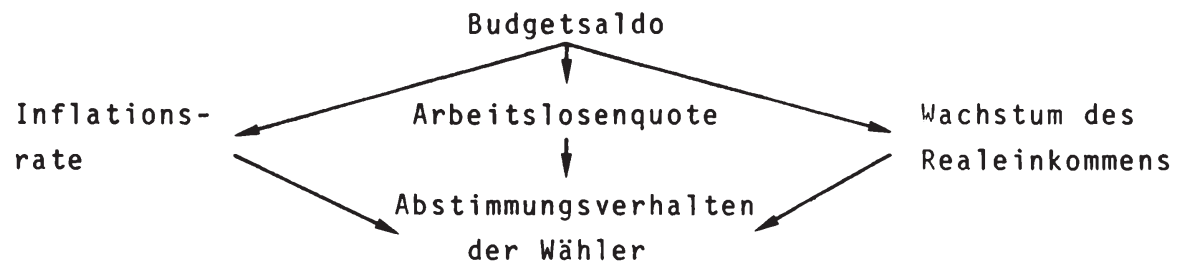

Das Schema zeigt, daB der mittelbare EinfluB des Budgetsaldos auf das Abstimmungsverhalten bei Annahme bestimmter funktionaler Beziehungen zwischen Budgetsalden und makroökonomischen Variablen über den Zusammenhang dieser Variablen mit dem Abstimmungsverhalten gemessen werden kann.

Dennoch muB deutlich auf die Grenzen dieser Vorgehenswe ise hingewiesen werden:

Zunächst einmal kann auf der Basis der bisher durchgeführten empirischen Untersuchungen lediglich der Zusammenhang der drei obengenannten makroökonomischen Größen mit dem Abstimmungsverhalten der Wähler analysiert werden. Ober die Auswirkungen der übrigen in Abschnitt III.1. diskutierten Effekte von Budgetsalden Iiegen keine empirischen Untersuchungen vor. Insbesondere die theoretische Analyse der Effekte der Zeitpräferenzen, der mit Budgetsalden verbundenen Verteilungswirkungen und der psychologischen Effekte auf den von den Wählern gewünschten Budgetsaldo entbehrt daher einer empirischen Grundlage.

An diesem Zustand düfte sich in naher Zukunft auch wenig ändern, da der Zusammenhang der Zeitpräferenzen und der Verteilungswirkungen mit dem Abstimmungsergebnis komplexer ist als im Falle von Inflationsrate, Arbeitslosenquote und Realeinkommenswachstum und daher empirisch kaum erfaBt werden kann. 
Weiterhin ist auch die Analyse des Zusammenhangs zwischen den drei zuletzt genannten makroökonomischen Aggregaten und dem Abstimmungsverhalten der Wähler umstritten. Politometrische Schätzungen drücken diesen Zusammenhang auf zweierlei Art und Weise aus. Ein Teil der Autoren untersucht den Zusammenhang der ökonomischen Variablen mit der Popularität der Regierung ${ }^{1)}$, ein anderer Teil den Zusammenhang mit dem Wahlergebnis ${ }^{2}$ ). Gegen beide Vorgehensweisen lassen sich jedoch mehr oder weniger gewichtige Einwände vorbringen.

Vor allem DINKEL hat auf den wesensmäßigen Unterschied zwischen Wahl- und Popularitätsdaten hingewiesen ${ }^{3)}$ und sich gegen eine Verwendung der letzteren ausgesprochen: ". . relevant ist in erster Linie die Beeinflussung des Wahlverhaltens durch wirtschaftliche Bedingungen. Popularitätsdaten sind keine vorweggenommenen Wahlergebnisse, sie folgen anderen Gesetzmäßigkeiten und können für die Ausrichtung der Politik einer Regierung U.U. eher irrefürend als hilfreich sein ${ }^{4}$."

Popularitätsdaten, die durch demoskopische Befragungen ("Wenn nächsten Sonntag Wahl wäre, welcher Partei würden Sie Ihre Stimme geben?") praktisch in beliebiger Höhe verfügbar gemacht werden können, fehlt vor allem der. Entscheidungscharakter der Wahl. Da Popularitätsbefragungen für den Befragten unverbindlich sind, $1 a ̈ B t$ er sich bei seiner Antwort auch eher von aktuellen Ereignissen der jüngsten Vergangenheit leiten, so daß das Ergebnis von der Auswahl des Befragungstages abhängt ${ }^{5}$ ). Dieser Einwand gewinnt vor allem

1) Vgl. Z.B. Goodhart/Bhansali (1970), Frey/Garbers (1971) und (1972), Miller/Mackie (1973), Kirchgäßner (1974) und (1976), S. 87 - 123, Frey/Schneider (1978) und (1978a), Jonung/Wadensjö (1980)

2) Vgl. Z.B. Kramer (1971), Stigler (1973), Lepper (1974), Arcelsus/Meltzer (1975) und (1975a), Bloom/Price (1975), Fair (1975) und (1978), Goodman/Kramer (1975), Tufte (1975)

3) Vgl. Dinkel (1977), S. $113 \mathrm{ff}$. Vgl. zu den folgenden Ausführungen auch Rattinger (1980), S. $53-56$

4) Dinkel (1977), S. 114

5) Vgl. Dinkel (1977), S. $129 \mathrm{f}$., vgl. auch derselbe (1978), S. 544 
dann an Gewicht, wenn von Monatsdaten auf Quartals- ${ }^{1}$ ) oder gar Jahresdaten übergegangen wird ${ }^{2}$.

Weiterhin kann das Ergebnis wie bei jeder demoskopischen Befragung durch den Einfluß des Interviewers und durch Auswahl der Stichprobe verfälscht werden ${ }^{3)}$. Durch die Tatsache, daß Popularitätsbefragungen nicht wie Wahlen geheim sind, besteht zudem die Möglichkeit, daß der Befragte seine wahre politische Meinung nicht preisgibt, was insbesondere bei Anhängern extremer Parteien der Fall sein diufte ${ }^{4}$ ).

Als weiteres gewichtiges Argument gegen die Verwendung von Popularitätsdaten wird die Existenz eines von der ökonomischen Entwicklung unabhängigen Popularitätszyklus angeführt ${ }^{5}$ ). Es wurde beobachtet, daß die Popularität der Regierung unmittelbar nach der Wahl kurzfristig ansteigt, dann stark abfällt und bis zum folgenden Wahltermin wieder auf den Normalstand ansteigt, während die Opposition von derartigen Bewegungen verschont bleibt. Der Anstieg der

1) So z.B. Frey (1975), Frey/Schneider (1978) und (1978a)

2) Vgl. Dinkel (1977), S. $130 \mathrm{f}$., der die Fragwürdigkeit der Verwendung von Vierteljahresdaten anhand zweier Methoden der Bildung dieser Daten aufzeigt. Vgl. auch derselbe (1978), S. 545 f.

3) Zu weiteren Faktoren, die die Umfrageergebnisse verzerren, vgl. z.B. Kaase (1972), S. 149.

4) So weist Dinkel (1977), S. 117 darauf hin, daß der Anteil der NPD-Wähler deshalb unterschätzt wurde, weil sich nicht alle NPD-Anhänger öffentlich zu dieser Partei bekennen wollten. Für die CDU/CSU-Wähler wurde dieser Effekt 1972 in abgeschwächter form auch vermutet.

5) Der "electoral cycle" wurde bereits von Goodhart/Bhansali (1970), S. 46 erwähnt. Vgl. auch Miller/Mackie (1973), Beetz (1978), S. $73 \mathrm{ff}$. sowie Dinkel (1977), S. $137 \mathrm{ff}$. und (1978), S. $551 \mathrm{ff}$. Dinkel analysiert hierbei die "relative Popularität", d.h. er vergleicht die tatsächliche Popularitätsentwicklung ex post mit der Popularitätsentwicklung, die sich aufgrund der Wahlergebnisse zweier aufeinanderfolgender Wahltermine hätte ergeben müssen, wenn die laufende Popularität dem Trend der Wahlergebnisse folgen würde. Gegen die Existenz eines Popularitätszyklus wendet sich Kirchgäßner (1974), S. 419 f., obwohl er auf S. 439 eigentlich das beschreibt, was andere Popularitätszyklus nennen. (Vgl. Dinkel (1977), S. 140.) An anderer Stelle räumen Kirchgäßner/ Schneider jedoch ein, daß die Ergebnisse Kirchgäßners auf die Existenz eines unabhängigen Wahlzyklus hindeuten. Vgl. Kirchgäßner/Schneider (1979), S. 103 
Popularität kurz nach der Wahl ist durch das Bestreben vieler Nicht-Regierungswähler, sich nachträglich den Gewinnern der Wahl anzuschlieBen, zu erklären ${ }^{1)}$. Sind die Regierungswähler im Laufe der Legislaturperiode mit der Politik der Regierung nicht zufrieden, so können sie ihre kurzfristige Verdrossenheit bei Popularitätsbefragungen kundtun, was zu einem Rückgang der Regierungspopularität führt, auch wenn viele der Unzufriedenen nicht beabsichtigen, eine andere Partei zu wählen. AuBerdem hat sich die Nachwahleuphorie vieler Nicht-Regierungswähler, die nach der Wahl zu den Gewinnern zählen wollten, inzwischen gelegt, so daß sie der Regierung wieder ihre sympathie entziehen. Dies bewirkt ebenfalls ein Absinken der Regierungspopularität. Mit Näherrücken der Wahl werden die Wähler wieder wahlbewußter. Der Tag der Entscheidung rückt näher und die Regierungswähler werden einzelne Maßnahmen, die sie der Regierung bei Popularitätsumfragen kurzfristig negativ angekreidet haben, wieder eher im Rahmen der gesamten Regierungspolitik sehen ${ }^{2}$. Als Folge davon wird die Regierungspopularität mit Näherrücken der Wahl wieder ansteigen ${ }^{3)}$. Schwankungen der Regierungspopularität dürfen somit nicht mit tatsächlichen Wechselbewegungen gleichgesetzt werden, da insbesondere die für die Wahl entscheidenden Wechselwähler ihre Wahlentscheidung erst kurz vor der Wahl treffen ${ }^{4}$ ).

DINKEL 5 ) hat in der Bundesrepublik Deutschland für den Zeitraum zwischen 1950 und 1974 anhand von Allensbacher Daten einen Popularitätszyklus festgestel1 $t^{6}$ ). Seine Ergebnisse deuten darauf hin,

1) Vgl. Dinikel (1977), S. 116

2) Vgl. derselbe (1978), S. 557

3) Die Tatsache, daß die Oppositionspopularität dieser zyklischen Entwicklung nicht ausgesetzt ist, zeigt, daß der "Verlust" der Regierungspartei nicht als tatsächliche Abwanderung zu interpretieren ist. Vgl. Dinkel (1977), S. $118 \mathrm{f}$.

4) Vgl. auch Freyer/Widmaier (1979), S. 172

5) Vgl. Dinkel (1977), S. $140 \mathrm{ff}$.

6) Vgl. hierzu für den Bereich der USA Stimson (1976). 
daß es nicht möglich ist, zwischen zyklischen Eigenschwankungen der Popularität und ökonomisch induzierten Popularitätszyklen zu trennen, so daß ein Kausalzusammenhang zwischen ökonomischen Variablen und Regierungspopularität fraglich bleibt ${ }^{1}$ ).

Der Nachweis eines eigenständigen Popularitätszyklus bedeutet jedoch nicht notwendig, daß von ökonomischen Variablen keinerlei EinfluB auf die Popularität der Regierung ausgeht. So haben GOODHART/BHANSALI im Vereinigten Königreich für den Zeitraum von 1947 bis 1968 neben dem "electoral cycle" auch einen signifikanten EinfluB der Inflation und der Arbeitslosigkeit auf die Regierungspopularität ${ }^{2)}$ festgestellt. KIRCHGASSNER/SCHNEIDER ${ }^{3)}$ und FREY/SCHNEIDER ${ }^{4}$ kommen be i ihrer Untersuchung des Zeitraums von Juli 1953 bis Oktober 1964 bzw. der Quartale 4/1959 bis 4/1974 zu ähnlichen Ergebnissen.

Weiterhin können die Meinungsforschungsinstitute versuchen, den mit der Popularitätsbefragung verbundenen verzerrenden Einflussen Rechnung zu tragen und die entsprechenden Verzerrungen auszugleichen. Schließlich ist die Verwendung von Popularitätsdaten auch

1) Vgl. Dinkel (1977), S. 143 sowie derselbe (1978), S. 551 ff. Die Existenz eines eigenständigen Popularitätszyklus wird in diesem zweiten Beitrag durch Vergleich des Popularitätszyklus mit der zeitlichen Abfolge der Wachstumszyklen in der Bundesrepublik Deutschland (vgl. hierzu Ott/Wagner (1973)) untermauert. Dinkel stellt fest, "daB die vier ökonomischen Spitzenjahre (die Jahre $1955,59,64$ und 68) allesamt mitten in die Legislaturperiode fallen und zu diesen Zeitpunkten die tatsächliche Popularität stets unterhalb der erwarteten Popularität der Regierung lag". (Vgl. (1978), S. 555)

2) Goodhart/Bhansali verwenden nicht die Regierungspopularität, sondern den "government lead", d.h. den Popularitätsvorsprung der Regierungspartei vor der Opposition, als abhängige Variable. Siehe auch Frey/Schneider (1978a).

3) Vgl. Kirchgäßner/Schneider (1979), S. 99 ff.

4) Vgl. Frey/Schneider (1978a). Diese Autoren stellen neben dem EinfluB des Wahlzyklus, der Arbeitslosenquote und der Inflationsrate auch einen signifikanten EinfluB des verfügbaren Realeinkommens auf den government lead fest. $\mathrm{Vgl}$. ebenda, S. $250 \mathrm{f}$. 
dann zu rechtfertigen, wenn Popularitätsbefragungen zwar keine "Quasiwahlen" sind, aber von den Politikern als solche angesehen werden 1 ).

Mit einem derartigen Verhalten der Politiker ist jedoch nicht während der gesamten Legislaturperiode, sondern eher im Zeitraum kurz vor der Wahl zu rechnen ${ }^{2)}$. Da der Regierung zudem von den verschiedenen Meinungsforschungsinstituten kein einheitlicher Popularitätsindex zur Verfügung steht, ist nicht damit zu rechnen, daß die Regierung ihre Politik bei jedem Absinken des Popularitätsindex ändert ${ }^{3)}$. Reagiert die Regierung aber nur auf Popularitätsdaten unmittelbar vor der Wahl, so büßen diese ihren Vorteil gegenüber Wahldaten, während eines überschaubaren Zeitraums in ausreichender Menge verfügbar zu sein, tendenziell ein.

Die in der nachstehenden übersicht zusammengestellten Ergebnisse ${ }^{4}$ ) können daher nur in begrenztem Umfang zur Oberprifung der BW-These herangezogen werden, zumal der quantitative EinfluB der einzelnen ökonomischen Variablen auch von der Wahl der zu erklärenden endogenen Politikvariablen ${ }^{5)}$ und dem zeitlichen Abstand der Popularitätsbefragungen ${ }^{6)}$ abhängt.

1) Vgl. Kirchgäßner/Schneider (1979), S. 92 sowie Gärtner (1980), S. 259

2) Vgl. auch Freyer (1979), S. 157

3) Vgl. Freyer/Widmaier (1979), S. 173

4) Vgl. Frey (1978b), S. 213, aber auch (1978a), S. 555. Bei dem letztgenannten Beitrag handelt es sich um die Zusammenfassung der Ergebnisse von Kirchgäßner (1976), Frey/Schneider (1978) und (1978a) sowie Frey (1978b).

5) So verwenden einige Autoren die Regierungspopularität, andere den Popularitätsvorsprung vor der Opposition als zu erklärende Popularitätsvariable. Vgl. hierzu auch Kirchgäßner/Schneider (1979), S. $100 \mathrm{ff}$. Sowie die Ausführungen über die Eignung von Wahldaten.

6) So wird die Einkommensvariable i.d.R. nur dann statistisch signifikant, wenn man Quartals- oder Jahresdaten verwendet. Vgl. Kirchgäßner/Schneider (1979), S. 102, Fußnote 71 und S. 104 


\begin{tabular}{l|ccc} 
POP & $\hat{\mathrm{p}}$ & ALQ & WRE \\
\hline $\begin{array}{l}\text { Bundesrepublik } \\
\text { Deutschland } \\
1957-1975\end{array}$ & $-1,5$ & \\
$1951-1975$ & $-0,71$ & $-1,7$ & 0,6 \\
& & & $0,4^{+}$ \\
$\begin{array}{l}\text { Vereinigtes } \\
\text { Königreich } \\
\text { 1959-1974 }\end{array}$ & $-0,61$ & $-6,01$ & 0,81 \\
USA & & & \\
$1953-1976$ & $-1,0$ & $-4,03$ & $0,52^{+}$
\end{tabular}

Tabelle 1: Der Einfluß ökonomischer Variablen auf die Regierungspopularität 1 )

Tabelle 1 ist zu entnehmen, daß sämtliche Untersuchungen einen starken EinfluB der Arbeitslosenquote ( $A L Q$ ) auf die Regierungspopularität (POP) feststellen. Nur in der Bundesrepublik Deutschland hat die Inflationsrate $(\hat{p})$ einen annähernd gleich starken Einfluß. Der EinfluB des Wachstums der verfügbaren Realeinkommen (WRE) war dagegen nur im Vereinigten Königreich und in einem Fall in der Bundesrepublik Deutschland statistisch signifikant ${ }^{2}$. Somit sprechen diese Untersuchungen eigentlich für die BW-These, da eine allzu stark ausgeprägte Anti-Inflations-Mentalität auch in der Bundesrepublik Deutschland nicht nachgewiesen werden kann. Aus den genannten Gründen können diese Ergebnisse jedoch nicht zur eindeutigen Bestätigung der BW-These herangezogen werden.

1) Die Werte geben an, um wieviel \% sich die Regierungspopularität bei einer e inprozentigen Anderung der entsprechenden ökonomischen Variablen ändert. Die mit + gekennzeichneten Koeffizienten waren statistisch nicht signifikant. Vgl. Frey (1978b), S. 214

2) Frey ((1978b), S. 214) führt dies auf das Vorliegen von Multikollinearität zurück. 
Auch die Verwendung von Wahldaten ist jedoch mit Problemen behaftet. Wahldaten sind, da Wahlen in der Regel nur alle drei bis füf Jahre stattfinden, in weitaus geringerem MaBe verfügbar als Popularitätsdaten. Zur Erzielung statistisch signifikanter Ergebnisse ist man jedoch darauf angewiesen, fur relativ lange Zeiträume vergleichbare Daten zur Verfügung zu haben. Dabei entsteht das Dilemma, daß mit zunehmender Länge des Untersuchungszeitraums die Vergleichbarkeit der Daten abnimmt ${ }^{1}$ ).

Dieses Dilemma ist der Grund dafür, daß bisher nur für den Bereich der Vereinigten Staaten von Amerika, also für ein Land, dessen politisches system während eines langen Zeitraums relativ stabil war ${ }^{2}$ ), einigermaßen befriedigende Ergebnisse bei der Schätzung von Wahlfunktionen erzielt werden konnten.

KRAMER $^{3)}$ hat die KongreBwahlen von 1896 bis 1964 analysiert und einen statistisch signifikanten Zusammenhang zwischen der Wachstumsrate des Realeinkommens und dem Stimmenanteil der Regierung festgestellt. Für andere ökonomische Variable konnte kein statistisch signifikanter Zusammenhang festgestellt werden ${ }^{4}$. Noch pessimistischer fallen die Ergebnisse von STIGLER ${ }^{5}$ aus, der aufgrund

1) Vgl. Beetz (1978), S. 71 sowie Roth (1973), S. 258. Auch Freyer/Widmaier ((1979), S. 171) weisen auf das Problem der Strukturkonstanz, d.h. der gleichbleibenden Bedeutung gleicher Indikatoren hin, die zumeist nicht gegeben ist. Die Autoren heben insbesondere auf die unterschiedliche Bedeutung der Arbeitslosigkeit in der Bundesrepublik Deutschland in den $50 \mathrm{er}$ Jahren und in den Jahren 1961 - 1966 und 1969 - $1971 \mathrm{ab}$.

2) Vgl. Dinkel (1977), S. 120

3) Vgl. Kramer (1971)

4) Vgl. aber Frey (1978a), S. 554, der in Kramers Untersuchung einen statistisch signifikanten EinfluB der Inflationsrate vorzufinden glaubt.

5) Vgl. Stigler (1973). Er bezieht sich auf die Untersuchung Kramers, wobei er einige von Kramer falsch beobachtete Daten korrigiert (vgl. ebenda, S. 160, insbesondere die FuBnote 3). Anstelle der prozentualen Realeinkommensänderung gegenüber dem Vorjahr verwendet Stigler allerdings eine Zeitdifferenz von zwei Jahren. "There is no naturally correct period ... but a two-year change ... seems a more reliable basis for appraisal of economic performance." Weiterhin rechnet er die Stimmen für kleine Parteien nicht der Opposition zu, sondern betrachtet sie als gegen das gesamte politische System gerichtet (vgl. Stigler (1973), S. 163). 
seiner Untersuchung die These aufstellt, daB die ökonomischen Bedingungen für die Ergebnisse der Kongreßwahlen keine Rolle spielen ${ }^{1)}$. Zur Unterstützung dieser These verwendet er jedoch ein Mode11, welches das von ihm als Ausgangspunkt verwendete KRAMER-Modell stark modifiziert. STIGLER geht davon aus, daB der Wähler derjenigen Partei seine Stimme gibt, von der er glaubt, daB sie im Durchschnitt ein größeres Realeinkommenswachstum realisieren wird. Bei der Prognose der alternativen Parteienleistungen bezüglich des Wachstumsziels legt der Wähler die bisherigen Leistungen der Parteien zugrunde, wobei diese Leistungen umso stärker abdiskontiert werden, je länger sie zurückliegen ${ }^{2}$ ). Auf der Basis dieser Oberlegungen testet STIGLER für die Zeit zwischen 1900 und 1932 (Republikaner uberwiegend an der Regierung) und zwischen 1932 1972 (Demokraten uberwiegend an der Regierung) in mehreren Modellen den statistischen Zusammenhang zwischen der Anderung des Stimmenanteils der Regierung und der mit dem Faktor $(1 / 1+r)^{t}$ gewichteten 3$)$ durchschnittlichen vergangenen Regierungsleistung. Er stellt in keinem Fall einen statistisch signifikanten Zusammenhang fest ${ }^{4}$. Bei Wahl eines modifizierten Ansatzes weist seine Schätzgleichung jedoch einen deutlichen EinfluB der Inflationsrate aus ${ }^{5}$ ).

Die Arbeit von STIGLER macht deutlich, daB ein geringfügiger Unterschied in der Theorie des Stimmverhaltens der Wähler zu stark unterschiedlichen empirischen Ergebnissen führen kann. In diesem Zusam-

1) Vgl. Stigler (1973), S. 160, vgl. auch Arcelsus/Meltzer (1975)

2) Stigler verweist in diesem Zusammenhang auf die Parallele zur Friedmanschen Konsumfunktion, deren unabhängige Variable auf ähnliche Weise ermittelt wird. Vgl. ebenda, S. 165

3) $r$ stellt die zur Abdiskontierung verwendete Diskontrate, $t$ Anzahl der zurück1 iegenden Perioden dar.

4) Vgl. Stigler (1973), S. 166

5) $\mathrm{Vgl}$. ebenda, S. 164. In diesem Ansatz verwendet Stigler wie Kramer den Stimmenanteil der Regierungspartei als zu erklärende Politikvariable. Der EinfluB der Inflationsrate auf das Wahlergebnis ist nicht mehr gegeben, wenn er die Anderung des Stimmenanteils als abhängige politische Variable verwendet. 
menhang ist die Arbeit von FAIR ${ }^{1}$ zu erwähnen, der ein allgemeines Modell des Wahlverhaltens zu formulieren versucht, das die meisten in anderen Arbeiten verwendeten Theorien als Spezialfälle enthält und das es erlaubt, diese Theorien gegeneinander zu testen. Allerdings analysiert FAIR nicht die Wahlen zum KongreB, sondern die Präsidentschaftswahlen ${ }^{2)}$. Er kommt zu dem Schluß, daß das Wahlergebnis vor allem durch die Wachstumsrate des Realeinkommens und die Anderung der Arbeitslosenquote beeinflußt wird. Dabei diskontieren die Wähler weiter zurückliegende Regierungsleistungen stark $a b, d . h$. Sie reagieren praktisch nur auf Einkommens- und Beschäftigungsänderungen unmittelbar vor der Wahl ${ }^{3}$ ).

So interessant die Arbeit von FAIR im Hinblick auf die Systematisierung alternativer Theorien des Stimmverhaltens ist, so schwach ist jedoch die empirische Absicherung. FAIR erkennt selbst die Einwände, die gegen seine empirischen Ergebnisse hervorgebracht werden müssen, basieren diese Resultate doch auf nur 16 Beobachtungswer$\operatorname{ten}^{4}$ ).

Somit bleibt festzuhalten, daB die BW-These durch bisher vorliegende empirische Untersuchungen weder bestätigt noch falsifiziert werden kann. Aufgrund der Widersprüchlichkeit der Ergebnisse empirischer Untersuchungen kann nicht einmal die Bewertung alternativer wirtschaftlicher Ziele seitens der Wähler eindeutig bestimmt werden. Mit einer endgültigen empirischen klärung ist in naher Zukunft aus mehreren Gründen auch nicht zu rechnen. Zunächst sind die theoretischen Zusammenhänge zwischen staatlichen Budgetsalden und ihren Wirkungen auf das Nutzenniveau der Wähler noch nicht ausreichend untersucht. Weiterhin besteht Uneinigkeit hinsichtlich der empirischen Datenbasis. Schließlich ist die methodische Vorgehensweise

1) Vgl. Fair (1978)

2) Vgi. hierzu ebenda, S. 165

3) $\mathrm{Vgl}$. ebenda, S. 171

4) Die Ergebnisse beziehen sich auf den Zeitraum von 1916 bis 1976. Vgl. ebenda, S. 166 und S. 171 
be i empirischen Untersuchungen zwischen den einzelnen Autoren noch umstritten, so daß keine vergleichbaren Ergebnisse zu erwarten sind.

3. Fazit: Die Auswirkungen von Budgetdefiziten auf das Abstimmungsverhalten der Wähler

Die theoretische Analyse der Auswirkungen staatlicher Budgetsalden auf das Nutzenniveau der Wähler zeigt, daß das votum der wähler für Defizite zwar unter den Annahmen von BW plausibel ist, daß aber mit Defiziten weitere, von den Autoren nicht berücksichtigte Effekte verbunden sind, die auch die gegensätzliche These stützen. Eine empirische oberprüfung der im einzelnen aufgezeigten Effekte stößt indessen auf Schwierigkeiten, da bisher lediglich der Einfluß der makroökonomischen Variablen Arbeitslosenquote, Inflationsrate und Realeinkommenswachstum auf das Abstimmungsverhalten der Wähler untersucht wurde. Die - mit Vorbehalten zu interpretierenden - empirischen Analysen des Zusammenhangs zwischen Regierungspopularität und den genannten makroökonomischen Größen legen den Schluß nahe, daB eine Erhöhung der Inflationsrate zu einem geringeren Popularitätsverlust für die Regierung führt als eine Erhöhung der Arbeitslosenquote, so daß die mit Defiziten verbundenen ökonomischen Effekte in der Tendenz für die BW-These sprechen. Vor allem in der Bundesrepublik Deutschland ist jedoch nicht auszuschließen, daß psychologische Effekte von einer bestimmten Höhe der Verschuldung an zu einer Ablehnung weiterer Budgetdefizite durch die Wähler führen. Verstärkt werden können die psychologischen Effekte durch aufkommende Zweifel der Wähler an der Eignung fiskalischer Maßnahmen der Nachfragesteuerung zur Beseitigung von Arbeitslosigkeit, die in einer Situation der Stagflation nicht unbegrüdet erscheinen ${ }^{1)}$. Wie stark diese gegenläufigen Effekte sind und wo die Grenze der Verschuldung liegt, bei deren Oberschreitung die Regierung mit

1) Dennoch kann niemand sagen, wie die Beschäftigungssituation ohne staatliche Defizite aussehen würde. 
einer Abwahl rechnen muB, kann allerdings zum gegenwärtigen Zeitpunkt nicht entschieden werden.

Im folgenden Kapitel wird nun die zweite Kernthese der Neoinstitutionalisten untersucht, die mit statilichen Defiziten verbundene Preissenkung für öffentliche Güter führe zu einer Erhöhung des Staatsanteils am Sozialprodukt. Während in Kapitel III untersucht wurde, ob Defizite zu einer Unterschätzung des Preises öffentlicher Leistungen führen, geht die These der Fiskalizlusion in Kapitel IV als A-priori-Annahme in die Analyse ein. 
IV. DIE AUSWIRKUNGEN EINES SINKENDEN STEUERPREISES AUF DAS WACHSTUM DES STAATSANTEILS

Im vorigen Kapitel wurde die von BW postulierte Tendenz zu Budgetdefiziten in Demokratien diskutiert. Dabei spielte u.a. die durch alternative Finanzierungsinstitutionen des Staates hervorgerufene Unterschätzung des "Preises" öffentlicher Leistungen eine Rolle. Dieses Kapitel untersucht die allokativen Konsequenzen eines sinkenden Steuerpreises ${ }^{1)}$. Es wird dabei unterstellt, daB Fiskalillusion vorliegt ${ }^{2}$.

Wie bereits erwähnt, stellen BW die These auf, daB die Wähler aufgrund des in ihrer Vorstellung gesunkenen Steuerpreises von den Politikern mehr öffentliche Güter verlangen und daB diese Mehrnachfrage für das Wachstum des Staatsanteils am Sozialprodukt in Demokratien verantwortlich ist.

Kritik an dieser These ist vor allem von BRUNNER geübt worden ${ }^{3)}$. BRUNNER räumt zwar ein, daB einiges für die Behauptung spreche, ein niedrigerer Steuerpreis führe zu einer Mehrnachfrage nach öffentlichen Gütern. Jedoch behandeln BW seiner Ansicht nach nur ein statisches Effizienzproblem, nämlich die Größe des öffentlichen Sektors im Vergleich zu einem durch Wählerpräferenzen und vollkommene Information definierten Optimum. Das relative wachstum des Regierungssektors, die kontinuierliche Zunahme des Staatsanteils am Sozialprodukt, können seiner Ansicht nach durch Fiskalillusion nicht erklärt werden.

1) Buchanan (1967a), S. 79 definiert den Steuerpreis als den Preis, den ein Individuum für eine zusätzliche Einheit eines öffentlichen Gutes in Form von Steuern bezahlen muß.

2) Die Möglichkeit, daß die Zensiten tatsächlich davon ausgehen können, nicht in vollem Umfang für die Rückzahlung eines Defizits aufkommen zu müssen, wurde bereits in III.1.4.9. erwähnt.

3) Vgl. Brunner (1978), S. 658 
Im folgenden soll die Kritik BRUNNERs etwas genauer untersucht werden. Dies geschieht in mehreren Schritten: Die Grundlage bildet eine von BUCHANAN bereits 1967 durchgeführte theoretische Analyse ${ }^{1)}$ der Interdependenzen zwischen Budgetdefiziten und der Nachfrage nach öffentlichen Guitern. Im Anschluß daran soll anhand eines dynamischen Modells zur Kritik BRUNNERs Stellung genommen werden. Der Realitätsgrad des Modells wird durch Aufhebung einzelner einschränkender Annahmen nach und nach erhöht.

Die gesamte Analyse unterstellt, daB die Nachfrage der Wähler nach öffentlichen Gütern tatsächlich von einem durch die Wähler errechneten "Steuerpreis" abhängt, d.h. daß die Wähler bei Forderung einer marginalen Ausgabenerhöhung mit einer marginalen Erhöhung ihrer individuellen Steuerbelastung und/oder der Budgetdefizite rechnen.

Alternativ kann unterstellt werden, daB die Wähler zwar die Notwendigkeit der obereinstimmung von Gesamtausgaben und Gesamteinnahmen des Staates erkennen, daß sie ihre individuelle steuerschuld aber als unabhängig von der Höhe des Gesamtbudgets und von der Höhe ihres Anteils an den Ausgaben betrachten ${ }^{2}$. Die Implikationen dieser Alternativannahme für die Bedeutung des Zusammenhangs von Budgetdefiziten und dem Wachstum des Statsanteils werden in $A b-$ schnitt IV.5. erörtert.

\section{Theoretische Grundlagen der BUCHANAN/WAGNER-These}

BUCHANAN geht in seinem Modell von einer Situation aus, in der be $i$ bisher ausgeglichenem Budget des Staates ein Budgetdefizit erforderlich wird, um einen befürchteten Rückgang der privaten Gesamt-

1) Vgl. Buchanan (1967a)

2) So die Annahme von Crain/Ekelund (1978), S. 818: "Just as firms (in a larger number setting) perceive no effects on price from increasing the ir individual output, so the individual voter may believe his own taxes to be unaffected by marginal increases in the quantity of government services he consumes." 
nachfrage zu kompensieren. Das Defizit werde durch Geldschöpfung finanziert $\left.{ }^{1}\right)$ und reiche gerade aus, die Nachfrage auf dem bisherigen Niveau zu halten ${ }^{2}$ ). Nun kann das erforderliche Defizit $D_{0}$ durch eine Erhöhung der Ausgaben bei konstanten Steuereinnahmen, durch Steuersenkungen bei konstanten Ausgaben oder durch eine Kombination von Ausgaben- und Besteuerungsmaßnahmen erreicht werden.

Sämtliche Maßnahmen führen annahmegemäß zu einer Senkung des Steuerpreises öffentlicher Leistungen, die bei einer Steuersenkung aber höher ausfällt als bei einer Ausgabenerhöhung, da letztere mit einer Ausdehnung des Angebots an öffentlichen Leistungen verbunden ist, so daß der für Preissenkungen zur Verfügung stehende Defizitbetrag auf mehr Giutereinheiten aufgeteilt werden muB. BUCHANAN fragt nun, wie die individuellen Präferenzen der Wähler - repräsentiert durch die Steuerpreiselastizität der Nachfrage des Medianwählers nach öffentlichen Leistungen - beschaffen sein müssen, damit genau die Menge an öffentlichen Gütern nachgefragt wird, die der Staat aufgrund seiner stabilisierungspolitischen Maßnahme bereitstellt.

Aus diesen Uberlegungen folgt, daß die Steuerpreiselastizität $E$ der Nachfrage nach öffentlichen Gütern bei jeder Kombination von Ausgabenerhöhung und Steuersenkung einen anderen Wert aufweisen muß, wenn die durch das Defizit veränderte Versorgung mit öffentlichen Gütern be $i$ den jeweils herrschenden Steuerpreisen den Wählerpräferenzen entsprechen sol13). Wie sich anhand von Abbildung 5 leicht zeigen $1 a ̈ B t$, setzt eine Steuersenkung bei konstanten Aus gaben eine Steuerpreiselastizität von Null, eine Ausgabenerhöhung bei konstanten Steuereinnahmen eine Steuerpreiselastizität von Eins voraus.

1) Vgl. Buchanan (1967a), S. 77. Durch die Annahme der Geldschöpfungsfinanzierung wird eine spätere Verzinsung und Tilgung des Defizits über Steuererhöhungen ausgeschlossen, so daß die Annahme einer "perceived tax-price reduction" für öffentliche Leistungen eher gerechtfertigt erscheint als im Falle einer Verschuldung auf dem Kapitalmarkt.

2) Vgl. ebenda, S. 80

3) Der Einfachheit halber wird im folgenden angenommen, in der betrachteten Volkswirtschaft werde nur ein öffentliches Gut bereitgestellt. 


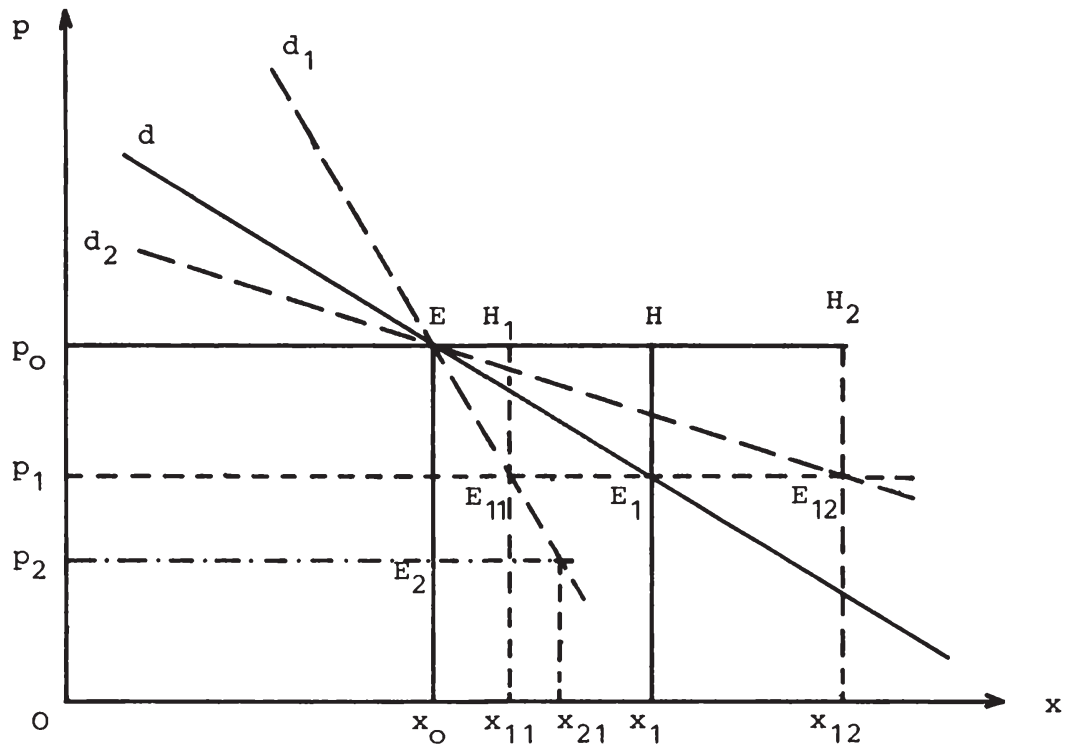

$\underline{\text { Abbildung }}^{1}{ }^{1}$ : Fiskalpolitik und individuelle Präferenzen

In Abbildung 5, in der die Ordinate den Steuerpreis $p$, die $A b-$ szisse die Menge $x$ des öffentlichen Gutes mißt, ist das staatliche Budget im Ausgangszustand be $i$ einem Volumen von $0 p_{0} E_{0}$ ausgeglichen. Das Defizit $D_{0}$ in Höhe von $P_{2} P_{0} E E_{2}$ führt zu einer Senkung des Steuerpreises auf $\mathrm{p}_{2}$. Nach dieser Preissenkung entspricht die vom Staat weiterhin bereitgestellte Menge $x_{0}$ nur dann den Präferenzen des Medianwählers, wenn die Steuerpreiselastizität gleich Null ist.

Im Falle einer Ausgabenerhöhung in Höhe von $D_{0}$ steigt das Ausgabevolumen auf $0 \mathrm{p}_{0} \mathrm{Hx}_{1}$, die bereitgestellte Menge steigt auf $x_{1}{ }^{2}$ ). Der neue Steuerpreis $p_{1} 1$ äßt sich graphisch durch den Schnittpunkt

1) Vgl. Buchanan (1967a), S. 79

2) Dabei sind $x_{0} E H x_{1}$ und $p_{2} p_{0} E E_{2}$ flächengleich. 
einer Geraden $\mathrm{OH}$ und $\mathrm{x}_{0} \mathrm{E}$ ermitteln ${ }^{1}$. Algebraisch ergibt er sich aus der Beziehung

(IV.1) $\quad p_{1}=\frac{p_{0} \cdot x_{0}}{x_{1}}$,

wobei $\mathrm{P}_{0} \cdot \mathrm{x}_{0}$ das konstante Steueraufkommen und damit die Kosten der Guitermenge $x_{1}$ repräsentiert. Die durch die staatliche Ausgabenerhöhung bedingte Preissenkung ist durch

(IV.2) $\Delta p=p_{1}-p_{0}$,

die Mengenerhöhung durch

(IV.3) $\Delta x=x_{1}-x_{0}$

definiert. Für die Steuerpreiselastizität

(IV.4) $\quad E=\frac{\Delta x}{\Delta p} \cdot \frac{p}{x}$

ergibt sich daher

(IV.5) $\quad E=\frac{x_{1}-x_{0}}{p_{1}-p_{0}} \cdot \frac{p}{x}$

oder unter Berücksichtigung von (IV.1)

(IV.5a) $\quad E=-\frac{x_{1}}{p_{0}} \cdot \frac{p}{x}$.

Für infinitesimal kleine Preis-und Mengenänderungen ergibt sich somit eine erforderliche Steuerpreiselastizität von

$(\text { IV. } 5 b)^{2)} \quad E=-1$.

1) Auf die Linie $\mathrm{OH}$ wurde in Abbildung 5 aus Gründen der Obersichtlichkeit verzichtet.

2) Vgl. Buchanan (1967a), S. 80 
Wir gehen nun einen Schritt weiter und fragen, welche Entwicklung in einem demokratischen staat zu erwarten ist, wenn die obengenannten Elastizitätserfordernisse nicht gegeben sind ${ }^{1}$. Auch dieser Gedankengang $1 a ̈ B t$ sich anhand von Abbildung 5 leicht nachvollziehen. Wir beschränken uns dabei auf den Fall der Defizitbildung durch eine Ausgabenerhöhung.

Ist $d_{1}$ die tatsächliche Nachfragefunktion, so wird bei einem Steuerpreis von $p_{1}$ nur die Menge $x_{11}$ nachgefragt. Hierfür wird von dem für Staatsausgaben vorgesehenen Betrag $0 \mathrm{p}_{0} \mathrm{Hx}_{1}$ nur der Teil $0 p_{0} H_{1} x_{11}$ benötigt, so daß die Differenz $x_{1} 1_{1} H_{1} x_{1}$ zum Abbau des Defizits verwendet werden kann. Die damit verbundene Erhöhung des Steuerpreises bewirkt einen weiteren Nachfragerückgang, der bei konstantem Steueraufkommen einen weiteren Abbau des Defizits und eine weitere Erhöhung des Steuerpreises zur Folge hat. Die anfängliche Mehrversorgung mit öffentlichen Gütern wird also wieder rückgängig gemacht.

Wird die tatsächliche Nachfrage nach dem öffentlichen Gut durch $d_{2}$ charakterisiert, fragt der Medianwähler mehr öffentliche Güter nach als durch $0 p_{0} H x_{1}$ finanziert werden kann. Kann der Wähler die von ihm gewünschte Menge $x_{12}$ durchsetzen, so ist ein zusätzliches Defizit in Höhe von $x_{1} H_{2} x_{12}$ erforderlich. Dieses Defizit senkt bei konstantem Steueraufkommen den Steuerpreis für das öffentliche Gut, so daB die Nachfrage weiter zunimmt. Ob dieser ProzeB der Defizit- und Budgetexpansion zu einer neuen Gleichgewichtsmenge unterhalb der Sättigungsmenge führt, kann nur im Rahmen einer dynamischen Analyse entschieden werden, der wir uns im folgenden Abschnitt zuwenden wollen.

Vorher soll kurz auf einen fiktiven Spezialfall eingegangen werden, den AMACHER/TOLLISON ${ }^{2}$ ) erwähnen: Sie untersuchen den Fall, daß

1) Vgl. Buchanan (1967a), S. $83 \mathrm{f}$.

2) Vgl. Amacher/Tollison (1974) 
die Regierung versucht, die Gesamtnachfrage durch die expansive Nachfragewirkung eines ausgeglichenen Budgets aufrechtzuerhalten (Haavelmo-Theorem) ${ }^{1}$ ). Dieser Fall ist deshalb interessant, weil er zeigt, welche dynamische Entwicklung sich andeutet, wenn den Politikern die Regel des Budgetausgleichs vorgegeben ist. Wie Abbildung 5 zeigt, ist eine Politik, die auf die expansive Wirkung einer Budgetverlängerung ( $z . B$. von $0 p_{0} E x_{0}$ auf $0 p_{0} H x_{1}$ ) abstellt, nur dann mit den Präferenzen des Medianwählers vereinbar, wenn EH die Nachfragefunktion repräsentiert, d.h. wenn die Steuerpreiselastizität gegen - $\infty$ strebt. Ist sie betragsmäßig kleiner als $\infty$, so fragt der Medianwähler aufgrund der Konstanz des Steuerpreises weiterhin die im Ausgangszustand bereitgestellte Menge nach, so daß sich langfristig keine Ausweitung der Versorgung mit öffentlichen Gütern durchsetzen $1 \ddot{a} B t^{2}$ ).

\section{Dynamische Analyse}

\subsection{Das Mode11}

In diesem Abschnitt sollen die von BW postulierten Entwicklungstendenzen der Nachfrage nach öffentlichen Gütern und des Staatsanteils am Bruttosozialprodukt anhand eines dynamischen Model ls überprüt werden. Zu diesem Zweck wird - unter Beibehaltung der Annahmen aus Teil IV.1. - das Modell einer Volkswirtschaft betrachtet, in der im Ausgangszustand $x_{0}=10$ Einheiten eines öffentlichen Gutes bereitgestellt werden. Die Kosten für die Bereitstellung einer Einheit des Gutes betragen $k=1$ und seien konstant. Die öffentlichen Güter werden durch Steuern in Höhe von $T_{0}=k \cdot x_{0}=10$ finanziert. Die Nachfrage des Medianwählers nach dem öffentlichen Gut ist durch die Funktion

1) Amacher/Tollison weisen selbst auf die begrenzte empirische Relevanz dieses Spezialfalls hin (vgl. Amacher/Tollison (1974), S. 108, Fußnote 2).

2) In diesem Zusammenhang ist auch die von Buchanan/Wagner vorgeschlagene Rückkehr zur verfassungsmäßig vorgeschriebenen Regel des Budgetausgleichs zu sehen, von der nur in Notfällen abgewichen werden darf. Vgl. Buchanan/Wagner (1977), S. $173 \mathrm{ff}$. 
$(\text { IV.6 })^{1)} \quad x_{t+1}^{n}=-f \cdot p_{t}+(f+10)$

gegeben.

Sie bringt den anhand von Abbildung 5 angedeuteten Sachverhalt zum Ausdruck, daB der Medianwähler auf eine Veränderung des Steuerpreises $p_{t}$ reagieren und in der nächsten Periode eine veränderte Menge $x_{t+1}$ des öffentlichen Gutes fordern wird. Weiterhin wird angenommen, daß die vom Medianwähler in jeder Periode gewünschte Menge des öffentlichen Gutes auch tatsächlich bereitgestellt wird:

$$
(\text { IV. 7 })^{2)} \quad x_{t}^{n}=x_{t} \text {. }
$$

Der vom Medianwähler wahrgenommene Steuerpreis $\mathrm{p}_{t}$ wird durch die Differenz der Stückkosten $k$ des öffentlichen Gutes und des Stückdefizits derselben Periode $D_{t} / x_{t}$ bestimmt, da das Defizit nicht verzinst und getilgt werden muß und der Medianwähler von einer Preissenkung ausgeht ${ }^{3)}$ :

(IV.8) $\quad p_{t}=k-\frac{D_{t}}{x_{t}}$.

Für $k=1$ ergibt sich

(IV.8a) $\quad p_{t}=1-\frac{D_{t}}{x_{t}}$.

1) Diese Form der Nachfragefunktion garantiert, daß bei $p=1$ unabhängig von der Größe von $f$ immer 10 Einheiten des öffentlichen Gutes nachgefragt werden.

2) $x_{t}$ bezeichnet die von den Politikern bereitgestellte Menge des öffentlichen Gutes.

3) Auch die übrigen Kosten des Defizits, die sich beispielsweise durch eine Erhöhung der Inflationsrate infolge der mit dem Defizit verbundenen Geldmengenerhöhung ergeben können, werden vom Medianwähler nicht wahrgenommen. Vgl. Z.B. Buchanan/Wagner (1978d), S. $20 \mathrm{f}$. 
Die Staatsausgaben $A_{t}$ dienen der Bereitstellung öffentlicher Güter

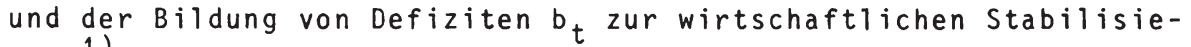
rung $^{1)}$ :

(IV.9) $\quad A_{t}=k \cdot x_{t}+b_{t}$.

Das in einer Periode insgesamt realisierte Budgetdefizit $D_{t}$ ergibt sich aus der Differenz der Staatsausgaben $A_{t}$ und der Steuern $T_{t}$, die wir als konstant annehmen wollen:

(IV.10) $T_{t}=T$,

(IV.11) $\quad D_{t}=A_{t}-T_{t}=k \cdot x_{t}+b_{t}-T$.

Da im Ausgangszustand kein Defizit besteht, beträgt der Steuerpreis $p_{0}=1$, so daB die bereitgestellte Menge gerade nachgefragt wird. In Periode 1 werde nun zur Vermeidung eines Rückgangs der Gesamtnachfrage ein stabilitätspolitisch bedingtes Defizit in Höhe von $b_{1}$ beschlossen, d.h. die Staatsausgaben in Periode 1 betragen $A_{1}=k \cdot x_{1}+b_{1}$. Diese Staatsausgaben sollen annahmegemäß zur Lösung der Stabilisierungsprobleme ausreichen und üben neben ihrer Wirkung auf die Höhe des Budgetsaldos keinen weiteren EinfluB auf das System aus 2 ).

Die im langfristigen Gleichgewicht bereitgestellte Menge des öffentlichen Gutes läßt sich nun durch Lösung der aus den Gleichungen (IV.6) bis (IV.11) ableitbaren inhomogenen Differenzengleichung 1. Ordnung

(IV.12) $\quad x_{t}-f\left(b_{t-1}-T\right) \cdot \frac{1}{x_{t-1}}-(f+10)=0$

1) Defizite zum Zwecke der wirtschaftlichen Stabilisierung seien im folgenden als "stabilitätspolitisch bedingte" Defizite bezeichnet.

2) Wir abstrahieren daher von Einkommenseffekten und untersuchen nur Substitutionseffekte auf die Nachfrage nach dem öffentlichen Gut. 
ermitteln.

Diese Gleichung strebt für $b_{t}=b=$ const. für $t \rightarrow \infty$ gegen den Grenzwert

(IV.13) $\bar{x}=\frac{f+10}{2}+\sqrt{\frac{(f+10)^{2}}{4}+f(b-T)}$,

wenn gilt:

(IV.14 $)^{1)}\left|\frac{f(b-T)}{x^{2}}\right|<1$

Bedingung (IV.14) ist im betrachteten Modell für alle relevanten $x$ erfiult.

Die dynamische Entwicklung des Systems soll durch ein Zahlenbeispiel anhand von Abbildung 6 veranschaulicht werden. Das Ausgangsgleichgewicht der betrachteten Volkswirtschaft sei durch $G(10,1)$ charakterisiert, wo die Nachfragefunktion nach dem öffentlichen Gut

(IV.6a) $\quad x_{t+1}^{a}=-10 p_{t}+20$

die Linie

(IV.15) p $x=10$

tangiert. Gleichung (IV.15) repräsentiert den geometrischen Ort aller Steuerpreise, die sich bei einem Steueraufkommen von $T=10$ bei alternativen Mengen von $x$ ergeben.

1) Nach dem Banachschen Fixpunktsatz hat die Funktion $f(x)=d+\frac{c}{x}$ für $d>0$, $c>0, x>0$ an der Stelle $\bar{x}=\frac{d}{2}+\sqrt{\frac{d^{2}}{4}+c}$ für $\left|\frac{c}{x^{2}}\right|<1$ einen anziehenden Fixpunkt, gegen den die Folge $x_{t}=f\left(x_{t-1}\right)$ für $x_{0}>0$ konvergiert. 


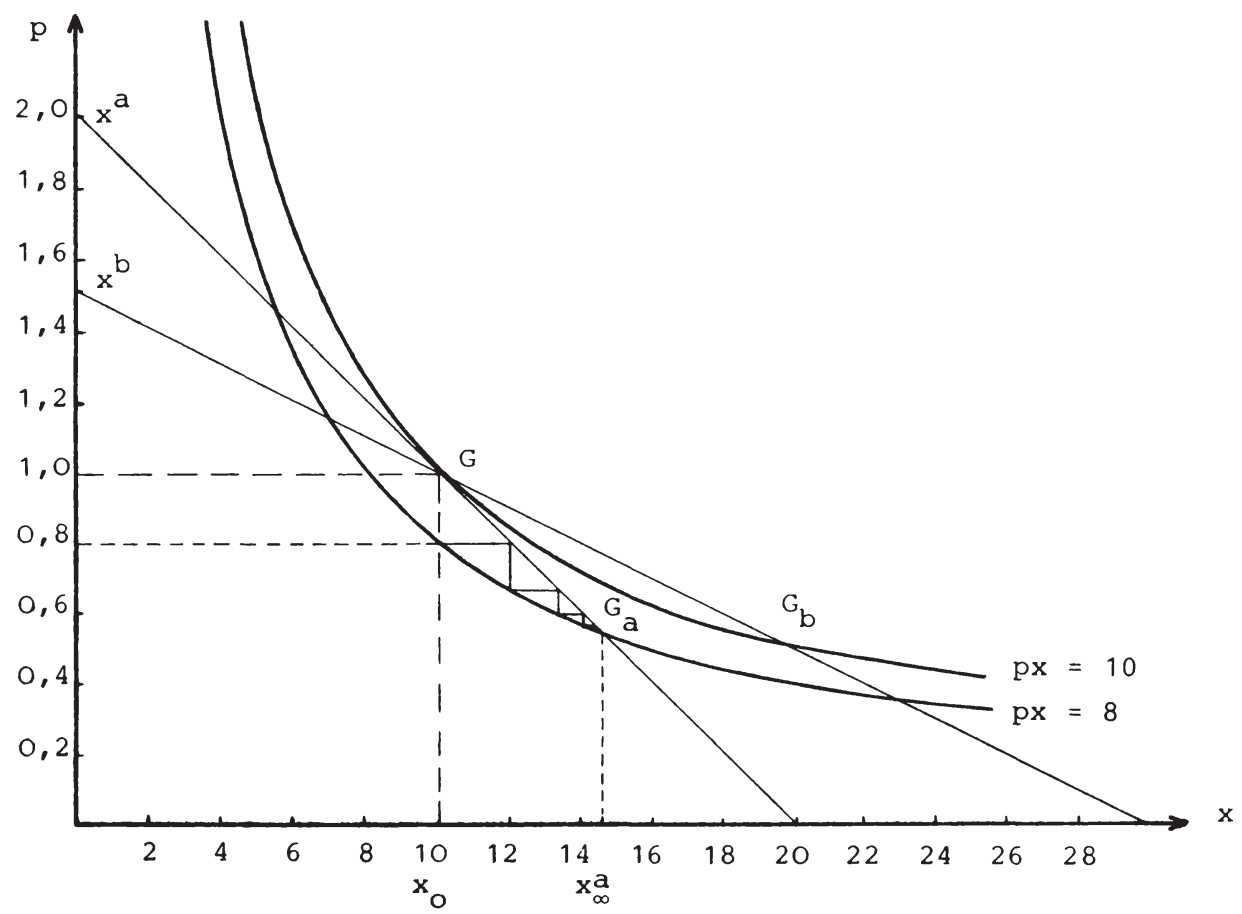

Abbildung 6: Dynamische Entwicklung der Nachfrage nach öffentlichen Giitern

Zur Aufrechterhaltung der Gesamtnachfrage werde ein stabilitätspolitisch bedingtes Defizit von $b_{1}=2$ beschlossen ${ }^{1}$. Dadurch sinkt der Steuerpreis auf $p_{1}=0,8 \mathrm{ab}$. Zu diesem Preis fordern die Wähler allerdings gemäß der Nachfragefunktion (IV.6a) 12 Einheiten des öf-

1) Ein stabilitätspolitisch bedingtes Defizit in Höhe von $20 \%$ des gesamten Steueraufkommens ist sicherlich etwas zu hoch. So hat die Bundesregierung im Rahmen ihrer "Maßnahmen zur Stärkung der Nachfrage und zur Verbesserung des Wirtschaftswachstums" im Jahre 1978 steuerliche Erleichterungen beschlossen, die sich für 1979 nur auf ca. $5 \%$ des gesamten Steueraufkommens belaufen ( $v g$ l. Bundesministerium der Finanzen (1978), S. 42 f.). Da sich prinzipiell für kleinere $b>0$ keine Unterschiede ergeben, scheint die Annahme von $b_{1}=2$ zu Illustrationszwecken legitim. 
fentlichen Gutes. Für die weitere dynamische Entwicklung ist nun entscheidend, ob das stabilitätspolitisch bedingte Defizit in Periode 2 noch aufrechterhalten oder ob es wieder abgebaut wird. Wird das stabilitätspolitisch bedingte Defizit beibehalten ${ }^{1}$, so ist bei konstantem Steueraufkommen und konstanten Stückkosten des öffentlichen Gutes ein zusätzliches Defizit von 2 GE erforderlich, um den Wünschen des Medianwählers nachzukommen. Aufgrund dieses weiteren Defizits vermindert sich allerdings der steuerpreis - wie sich auf der Kurve

(IV.16) p $\quad x=8$

be $i x_{2}=12$ ablesen $1 \ddot{a} B t^{2}$ - auf $p_{2}=0, \overline{6}$, was eine weitere Nachfragesteigerung bewirkt. Da auch diese Nachfrage annahmegemäB befriedigt wird, muB c.p. ein weiteres Defizit gebildet werden usw. Der Proze $B$ kommt allerdings be $i x_{\infty}^{a}=14,47$ und $p_{\infty}^{a}=0,553$ zum Stillstand, da die Nachfragekurve in diesem Punkt von der Linie (IV.16) geschnitten wird. Ein aus Stabilitätsgründen erforderliches Defizit hat damit zu einer Erhöhung des Angebots an öffentlichen Gütern um 4,47 Einheiten gefüht. Tabelle 2 gibt den Anpassungsprozeß zahlenmäßig wieder.

Wird das stabilitätspolitisch bedingte Defizit jedoch in Periode 2 wieder abgebaut, was durch das Vertrauen in einen sich durch Wirkung des Akzelerators selbst tragenden Aufschwung erklärt werden könnte, kommt es zu keiner Ausdehnung des Staatsanteils. Dies $1 a ̈ B t$ sich anhand von Tabelle 3 und Abbildung 6 leicht zeigen: Wie im obigen Beispiel sinkt der Steuerpreis zunächst auf $p_{1}=0,8$, was

1) Hierfür spricht die traditionelle Multiplikatortheorie, nach der eine nachhaltige Erhöhung des Volkseinkommens (bzw. eine Vermeidung eines Einkommensrückgangs) nur dann gesichert werden kann, wenn die Erhöhung der autonomen Ausgaben dauerhaft ist. Vgl. Z.B. Ott (1963), S. $159 \mathrm{ff}$.

2) Die Kurve $p: x=8$ ist der geometrische Ort aller Steuerpreise, die sich bei einem stabilitätspolitisch bedingten Defizit von $b_{1}=2$ bei alternativen Mengen unter der Annahme ergeben, daß das stabilitätspolitisch bedingte Defizit aufrechterhalten wird. 


\begin{tabular}{|c|c|c|c|c|c|c|c|}
\hline$t$ & $\begin{aligned} A_{t}^{+} & =k x_{t} \\
& =x_{t}\end{aligned}$ & $b_{t}$ & $A_{t}=x_{t}+b_{t}$ & $T$ & $D_{t}=A_{t}-T$ & $p_{t}=1-\frac{D_{t}}{x_{t}}$ & $x_{t+1}=-10 p_{t}+20$ \\
\hline 0 & 10 & 0 & 10 & 10 & 0 & 1 & 10 \\
\hline 1 & 10 & 2 & 12 & 10 & 2 & 0,8 & 12 \\
\hline 2 & 12 & 2 & 14 & 10 & 4 & $0, \overline{6}$ & $13, \overline{3}$ \\
\hline 3 & $13, \overline{3}$ & 2 & $15, \overline{3}$ & 10 & $5, \overline{3}$ & 0,6 & 14 \\
\hline 4 & 14 & 2 & 16 & 10 & 6 & 0,57 & 14,286 \\
\hline 5 & 14,286 & 2 & 16,286 & 10 & 6,286 & 0,56 & 14,4 \\
\hline - & $\cdot$ & - & $\cdot$ & - & $\cdot$ & $\cdot$ & - \\
\hline$\cdot$ & • & - & • & • & • & • & - \\
\hline$\cdot$ & • & - & • & • & • & • & • \\
\hline$\infty$ & 14,47 & 2 & 16,47 & 10 & 6,47 & 0,553 & 14,47 \\
\hline
\end{tabular}

Tabelle 2: Dynamische Entwicklung bei Beibehaltung des stabilitätspolitisch bedingten Defizits

\begin{tabular}{|c|c|c|c|c|c|c|c|}
\hline$t$ & $\begin{aligned} A_{t}^{+} & =k x_{t} \\
& =x_{t}\end{aligned}$ & $b_{t}$ & $A_{t}=x_{t}+b t$ & $T$ & $D_{t}=A_{t}-T$ & $p_{t}=1-\frac{D_{t}}{x_{t}}$ & $x_{t+1}=-10 p_{t}+20$ \\
\hline 0 & 10 & 0 & 10 & 10 & 0 & 1 & 10 \\
\hline 1 & 10 & 2 & 12 & 10 & 2 & 0,8 & 12 \\
\hline 2 & 12 & 0 & 12 & 10 & 2 & $0,8 \overline{3}$ & $11, \overline{6}$ \\
\hline 3 & $11, \overline{6}$ & 0 & $11, \overline{6}$ & 10 & $1, \overline{6}$ & 0,857 & 11,429 \\
\hline 4 & 11,429 & 0 & 11,429 & 10 & 1,429 & 0,875 & 11,25 \\
\hline 5 & 11,25 & 0 & 11,25 & 10 & 1,25 & $0, \overline{8}$ & $11, T$ \\
\hline - & - & • & • & • & • & - & • \\
\hline - & - & • & - & • & • & • & - \\
\hline - & - & - & - & - & - & - & . \\
\hline$\infty$ & 10 & 0 & 10 & 10 & 0 & 1 & 10 \\
\hline
\end{tabular}

Tabelle 3: Dynamische Entwicklung bei Abbau des stabilitätspolitisch bedingten Defizits in Periode 2 
zu einer Bereitstellung von 12 Einheiten des öffentlichen Gutes führt. Durch Abbau des stabilitätspolitisch bedingten Defizits steigt der Steuerpreis jedoch in Periode 2 wieder auf $0,8 \overline{3}$ an, was zu einem Nachfragerückgang auf $11, \bar{\sigma}$ führt usw.

In Abbildung 6 ist für die dynamische Entwicklung nicht mehr die Kurve $p \cdot x=8$, sondern wegen des Abbaus des Defizits die Kurve $p \cdot x=10$ maßgebend. Der Anstieg des Steuerpreises und der dadurch bewirkte Nachfragerückgang führen daher zu einem kontinuierlichen Abbau des Defizits.

Auch im Falle eines Abbaus des stabilitätspolitisch bedingten Defizits in einer späteren Periode liegt das neue Gleichgewicht bei der Ausgangsmenge $x=10$. Somit kommt es bei Geltung der Nachfragefunktion (IV.6a) nur dann zu einer dauerhaften Erhöhung der Versorgung mit öffentlichen Gütern, wenn das stabilitätspolitisch bedingte Defizit für alle Zeiten beibehalten wird.

Ist die Nachfragefunktion nach dem öffentlichen Gut jedoch elastischer als (IV.6a), so erhöht sich aufgrund des stabilitätspolitisch bedingten Defizits in Periode 1 die Gleichgewichtsmenge auch dann, wenn das stabilitätspolitisch bedingte Defizit in einer späteren Periode wieder abgebaut wird. Abbildung 6 zeigt, daB die beispielhaft gewählte - Nachfragefunktion

(IV.6b) $\quad x_{t+1}^{b}=-20 p_{t}+30$

rechts von $G$ oberhalb der Kurve $p \cdot x=10$ verläuft. Sie ist folglich so elastisch, daß die durch die Preissenkung auf 0,8 bedingte Nachfragesteigerung mit einem Steueraufkommen von $T=10$ nicht mehr finanziert werden kann. Es existiert daher rechts von $G$ ein Schnittpunkt $G_{b}$ der Nachfragekurve $x^{b}$ mit $p \cdot x=10$, so daB sich die Versorgung mit öffentlichen Giutern trotz des Abbaus des stabilitätspolitisch bedingten Defizits auf $x_{\infty}^{b}=20$ erhöht. 


\subsection{Folgerungen aus dem dynamischen Modell}

Die dynamische Analyse zeigt, daß sich bei genügend elastischer Nachfrage nach dem öffentlichen Gut eine Erhöhung des Angebots an öffentlichen Gütern ableiten läßt. Welche Schlußfolgerungen können hieraus für die Entwicklung des Staatsanteils am Sozialprodukt gezogen werden?

An dieser Stelle muB untersucht werden, wie sich das Sozialprodukt entwickelt. Durch die von BUCHANAN übernommene Annahme, das stabilitätspolitisch bedingte Defizit reiche gerade aus, um den zu befürchtenden Mangel der Gesamtnachfrage auszugleichen, wird postuliert, daB von seiten der privaten Nachfrage keine weiteren Wirkungen auf das Volkseinkommen ausgehen. Dennoch können wir nicht von einem konstanten Volkseinkommen ausgehen, da sich ja die Staatsausgaben laufend verändern.

Werden zum Volkseinkommen $Y_{0}$ die jeweiligen Veränderungen der Staatsausgaben gegenüber dem Ausgangszustand addiert, so ergibt sich die jeweilige Statsquote als

$$
a_{t}=\frac{A_{t}}{\gamma_{0}+\Delta A_{t}}
$$

mit

(IV.18) $\quad \Delta A_{t}=A_{t}-A_{0}$

bzw.

(IV.18a) $\quad \Delta A_{t}=A_{t}-10$.

Tabelle 4 gibt einen Oberblick über die auf dieser Grundlage ermittelten Staatsquoten. 


\begin{tabular}{|c|c|c|c|c|c|c|c|c|}
\hline $\begin{array}{l}\text { Relevante } \\
\text { Nachfrage } \\
\text { funktion }\end{array}$ & $b_{\infty}$ & $\mathrm{Y}_{0}$ & $A_{\infty}$ & $\begin{array}{l}\Delta A= \\
A_{\infty}-10\end{array}$ & $Y_{0}+\Delta A$ & $\begin{array}{l}Q_{\infty}= \\
\frac{A_{\infty}}{Y_{0}+\Delta A} \\
\text { in } \%\end{array}$ & $\begin{array}{l}Q_{0}= \\
A_{0} \\
\frac{Y_{0}}{i n} \%\end{array}$ & $\begin{array}{l}\Delta 0 \\
\text { in Pro- } \\
\text { zent- } \\
\text { punkten }\end{array}$ \\
\hline$x_{t}^{a}=-10 p_{t-1}+20$ & 2 & 30 & 16,47 & 6,47 & 36,47 & 45,2 & $33, \overline{3}$ & $+11,9$ \\
\hline$x_{t}^{a}=-10 p_{t-1}+20$ & 0 & 30 & 10 & 0 & 30 & $33, \overline{3}$ & $33, \overline{3}$ & \pm 0 \\
\hline$x_{t}^{b}=-20 p_{t-1}+30$ & 2 & 30 & 25,06 & 15,06 & 45,06 & 55,6 & $33, \overline{3}$ & $+22,3$ \\
\hline$x_{t}^{b}=-20 p_{t-1}+30$ & 0 & 30 & 20 & 10 & 40 & 50,0 & $33, \overline{3}$ & $+16, \overline{6}$ \\
\hline
\end{tabular}

Tabelle 4: Obersicht über die Veränderung der Staatsquote

Es wird deutlich, daB sich der Anteil der Staatsausgaben am Sozialprodukt in den Fällen, in denen das stabilitätspolitisch bedingte Defizit beibehalten wird, erhöht. Aber auch im Falle eines Abbaus dieses Defizits erhöht sich der Staatsanteil bei genügend steuerpreiselastischer Nachfrage.

Unter den restriktiven Annahmen des Modells stellt daher die These von B.W eine theoretische Erklärungsmöglichkeit für das relative Wachstum der Staatsausgaben dar. Der Einwand BRUNNERs, BW behandelten lediglich ein statisches Effizienzproblem, läBt sich durch Dynamisierung des Modells unter den dargestellten Annahmen entkräften.

Dennoch stellt sich aufgrund der sehr restriktiven Annahmen des Modells die Frage, ob die von BW gelieferte theoretische Erklärungsmöglichkeit empirische Relevanz besitzt.

Im Hinblick auf die empirische Relevanz unseres Modells ist insbesondere zu klären, ob erwartet werden kann, daB ein stabilitätspo- 
litisch bedingtes Defizit genügend lange aufrechterhalten wird ${ }^{1}$, bzw. ob die Nachfrage nach öffentlichen Gütern eine genügend hohe Steuerpreiselastizität aufweist, so daB sich auch be i einem eventuellen Abbau des stabilitätspolitisch bedingten Defizits eine Ausdehnung des Statsanteils ergibt. Vorher ist jedoch zu überprüfen, ob sich wesentliche Aussagen des Modells ändern, wenn wir einige der restriktiven Annahmen aufgeben bzw. das Modell erweitern. Zu berücksichtigen sind in erster Linie durch Verschuldung im privaten Sektor finanzierte Budgetdefizite verbunden mit einer zumindest teilweisen Wahrnehmung zukünftiger Zins- und Tilgungsverbindlichkeiten seitens der Wähler. Weiterhin wurden bisher mögliche Preissteigerungen, die aus der Mehrnachfrage nach öffentlichen Gütern resultieren können ${ }^{2}$, vernachlässigt. Schließlich ist eine realistischere Entwicklung des Volkseinkommens und des Steueraufkommens zu beruicksichtigen.

Durch Verschuldung im privaten Sektor finanzierte Defizite lassen sich berücksichtigen, indem wir in die steuerpreisfunktion (IV.8) eine Größe a einführen ${ }^{3)}$, die das AusmaB der Wahrnehmung zukünftiger Steuerverbindlichkeiten zum Ausdruck bringt:

(IV.8b) $\quad p_{t}=k-\frac{a \cdot D_{t}}{x_{t}}$

1) Obwohl das Modell verlangt, daB das stabilitätspolitisch bedingte Defizit bis $t \rightarrow \infty$ aufrechterhalten wird, verlangen wir nur eine "genügend lange" Beibehaltung des Defizits. Der dynamische ProzeB führt schon nach wenigen Perioden zu einer Versorgung mit öffentlichen Gütern nahe dem Grenzwert. Inzwischen können zahlreiche modellexogene Faktoren zu einer Veränderung des stabilitätspolitisch bedingten Defizits und damit zu einem weiteren dynamischen Proze $B$ führen, der den ursprünglichen ProzeB überlagert. Von einer genügend langen Beibehaltung des stabilitätspolitisch bedingten Defizits werden wir daher dann reden, wenn das Defizit bis zur nächsten fiskalpolitischen Maßnahme aufrechterhalten wird.

2) $k$ ist also nicht konstant.

3) In a sollen auch eventuelle Abneigungen der Wähler gegen Staatsschulden zum Ausdruck kommen. Sie führen zu einer Verringerung von a und über die damit verbundene Steuerpreissteigerung zu einer verminderten Nachfrage nach öffentlichen Leistungen. 
Eine vollständige Wahrnehmung künftiger Zins- und Tilgungsverbindlichkeiten wäre bei $a=0$ gegeben. Für $0<a<1$ werden die $\mathrm{Zu}-$ kunftsverbindlichkeiten teilweise wahrgenommen ${ }^{1}$ ).

Bei Verwendung von (IV.8b) führt das Gleichungssystem auf die Differenzengleichung

$$
\text { (IV.12a) } \quad x_{t}-a f\left(b_{t-1}-T\right) \cdot \frac{1}{x_{t-1}}-(a f+10)=0
$$

Diese Gleichung hat für $a=0$, also bei vollständiger Wahrnehmung der mit dem Defizit verbundenen Zins- und Tilgungsverbindlichkeiten die Lösung $x_{t}=10$, d.h. der Staatsanteil erhöht sich nicht. Für $0<a<1$ ergeben sich gegenüber dem durch (IV.12) charakterisierten Prozeß keine prinzipiellen Anderungen. Es kommt zu einer Erhöhung des Staatsanteils, die allerdings umso geringer ausfällt, je kleiner a ist. Stabilitätspolitisch bedingte Defizite können also auch dann zu einer Erhöhung des Staatsanteils am Sozialprodukt führen, wenn sie durch Verschuldung im privaten Sektor finanziert werden, die mit der Verschuldung verbundenen Zukunftsverbindlichkeiten aber von den wählern nicht vollständig berücksichtigt werden.

Steigende Preise für öffentliche Güter führen zwar zu einer nominal höheren Bereitstellung des öffentlichen Gutes und zu einem höheren Budgetdefizit, die bereitgestellte Menge des öffentlichen Gutes ändert sich jedoch nicht. Dieses (bei oberflächlicher Betrachtung vielleicht erstaunliche) Ergebnis läßt sich aus Gleichung (IV.12) sofort erkennen ( $k$ ist in der Gleichung nicht enthalten) und ist durch die Definition des Steuerpreises als Differenz zwischen tatsächlichen Stückkosten $k$ und Stückdefizit $D_{t} / x_{t}$ bedingt. Da ein steigender Angebotspreis sowohl die Stückkosten als auch das Stückdefizit um denselben Betrag erhöht, bleibt der Steuerpreis konstant

1) Werden die zukünftigen Steuerverbindlichkeiten überschätzt oder besteht eine starke Defizitabneigung, so ist $a<0$. 
und die nachgefragte Menge ändert sich nicht. Allerdings steigen die monetären Staatsausgaben, das Budgetdefizit und der durch das Verhältnis der nominalen Staatsausgaben zum Bruttosozialprodukt gemessene Staatsanteil ${ }^{1}$.

Tabelle 5 gibt die dynamische Entwicklung für eine beispielhaft gewählte Angebotsfunktion

(IV.19) $\quad k_{t}=0,5+0,05 x_{t}$

und Beibehaltung des stabilitätspolitisch bedingten Defizits wieder.

Die Berücksichtigung einer realistischeren Einkommens- und Steueraufkommensentwicklung bleibt dem folgenden Abschnitt vorbehalten.

2.3. Berücksichtigung des Staatsausgabenmultiplikators und einkommensabhängiger steuern

Das Modell ging bisher von der Annahme aus, das staatliche Budgetdefizit reiche gerade aus, um einen befürchteten Mangel an privater Nachfrage zu kompensieren. Der hinter dieser Wirkung stehende dynamische Proze $B$ wurde nicht analysiert.

Nunmehr wird versucht, diesen Mangel zu beheben, indem die Wirkung des Staatsausgabenmultiplikators bei einkommensabhängigen Steuern berücksichtigt wird. Das Modell wird hierzu durch mehrere Gleichungen erweitert: Das Volkseinkommen $Y_{t}$ sei durch

(IV.20) $\quad V_{t}=C_{t}+A_{t}+I_{t}$

als Summe aus Konsum $C_{t}$, Staatsausgaben $A_{t}$ und Investitionen $I_{t}$ definiert, wobei der Konsum vom verfügbaren Einkommen der Vorperiode abhänge

1) Selbstverständlich kạnn aus dieser nur durch Preisänderungen bedingten Erhöhung des Staatsanteils nicht auf einen erhöhten Staatseinfluß gegenüber dem privaten Sektor geschlossen werden (die Prämissen des Modells lassen eher die umgekehrte Vermutung zu). Zur Problematik der Messung des Staatseinflusses anhand von "Staatsquoten" vgl. Z.B. Folkers (1979), S. 459. 


\begin{tabular}{|c|c|c|c|c|c|c|c|c|c|c|}
\hline$t$ & $A_{t}^{+}=k_{t} \cdot x_{t}$ & b & $A_{t}=A_{t}^{+}+b$ & $\bar{T}$ & $D_{t}=A_{t}-\bar{T}$ & $p_{t}=k_{t}-\frac{D_{t}}{x_{t}}$ & $x_{t+1}=-10 p_{t}+20$ & $\begin{array}{l}\Delta x \\
=x_{t+1}-x_{0}\end{array}$ & $\begin{array}{l}k_{t+1} x_{t+1} \\
=0,5+\frac{t^{20}}{20}\end{array}$ & $k_{t+1} \cdot x_{t+1}$ \\
\hline 0 & 10 & 0 & 10 & 10 & 0 & 1 & 10 & 0 & 1 & 10 \\
\hline 1 & 10 & 2 & 12 & 10 & 2 & 0,8 & 12 & 2 & 1,1 & 13,2 \\
\hline 2 & 13,2 & 2 & 15,2 & 10 & 5,2 & 0,66 & $13, \overline{3}$ & $3, \overline{3}$ & $1,1 \overline{6}$ & 15,5 \\
\hline 3 & 15,5 & 2 & 17,5 & 10 & $7, \overline{5}$ & 0,6 & 14 & 4 & 1,2 & 16,8 \\
\hline 4 & 16,8 & 2 & 18,8 & 10 & 8,8 & 0,57 & 14,286 & 4,286 & 1,21 & 17,29 \\
\hline 5 & 17,29 & 2 & 19,29 & 10 & 9,29 & 0,56 & 14,4 & 4,4 & 1,22 & 17,57 \\
\hline • & $\cdot$ & - & • & $\cdot$ & • & • & $\cdot$ & • & • & • \\
\hline - & $\cdot$ & $\cdot$ & - & $\cdot$ & • & - & • & - & • & • \\
\hline - & - & - & - & - & - & - & - & - & $\cdot$ & - \\
\hline$\infty$ & 17,7 & 2 & 19,7 & 10 & 9,7 & 0,553 & 14,47 & 4,47 & 1,223 & 17,7 \\
\hline
\end{tabular}

Tabelle 5: Die dynamische Entwicklung bei steigenden Grenzkosten 
$(I V .21)^{1)} \quad c_{t}=c(1-\tau) r_{t-1}$.

Die Investitionen seien im Ausgangszustand durch

(IV.22) $I_{0}=I+m$,

in den Folgeperioden durch

(IV.23) $\quad I_{t}=I \quad$ für $t=1,2, \ldots$

gegeben.

Das Steueraufkommen sei durch die Funktion

(IV.10a) $\quad T_{t}=\tau \gamma_{t}$

bestimmt. Das erweiterte Modell besteht dann aus den Gleichungen (IV.6) bis (IV.9), (IV.10a), (IV.11) und (IV.20) bis (IV.23). Es läßt sich auf die Differenzengleichung

(IV.24) $\quad x_{t}=f+10-f \tau k+f \frac{(1-\tau) b-\tau I}{x_{t-1}}-f \frac{\tau c(1-\tau) y_{t-2}}{x_{t-1}}$

reduzieren.

Hieraus errechnet sich ein Grenzwert

(IV.25) $\quad x=\frac{(f+10) s_{\tau}-f \tau k}{2 s_{\tau}}+\sqrt{\left.\frac{(f+10) s_{\tau}-f \tau k}{2 s_{\tau}}\right\}^{2}+\frac{f b s_{\tau}-\tau(I+b)}{s_{\tau}}}$,

wobe $i$

$\mathbf{s}_{\tau}=1-c(1-\tau)$

1) c stellt die marginale Konsumquote, $\tau$ den durchschnittlichen, auf das Einkommen bezogenen Steuersatz dar. 
die effektive marginale Sparquote darstellt.

Die dynamische Entwicklung des Systems wurde für die Nachfragefunktionen

(IV.6c) $\quad x_{t+1}^{c}=-p_{t}+11$

und

(IV.6d) $\quad x_{t+1}^{d}=-50 p_{t}+60$

simuliert. Für die Parameter $k, Y_{0}$, $c$ und $\tau$ wurden folgende Werte angenommen

$k=1$

$\mathrm{Y}_{0}=30$

$c=0,75$

$\tau=0, \overline{3}$,

so da $B$ die effektive marginale Konsumquote $c(1-\tau)$ den Wert 0,5 aufwe ist.

Die Investitionen haben in $t_{0}$ eine Höhe von $I_{0}=5$ und fallen in den folgenden Perioden auf $T=3 \mathrm{ab}$. Diese Nachfragelücke wird durch ein stabilitätspolitisch bedingtes Defizit in Höhe von $b_{1}=2$ ge schlossen. Die Tabellen 6 und 7 geben die dynamische Entwicklung wieder, wobe $i$ in Teil a) von einer Beibehaltung, in Teil b) von einem Abbau des stabilitätspolitisch bedingten Defizits ausgegangen wird.

Aus den Tabellen 6 und 7 ist ersichtlich, daB die Werte der ökonomischen Variablen im Zeitablauf schwanken. Diese Schwankungen nehmen mit steigender Steuerpreiselastizität der Nachfrage zu. Bei betragsmäßig sehr hohen Steuerpeiselastizitäten liefert das System gar ökonomisch nicht mehr sinnvolle Ergebnisse. Legen wir beispielsweise die Nachfragefunktion 
a)

\begin{tabular}{|c|l|l|l|l|c|c|c|c|c|c|c|}
\hline$t$ & $k x_{t}$ & $b_{t}$ & $A_{t}=k x_{t}+b_{t}$ & $I_{t}$ & $C_{t}=\frac{1}{2} Y_{t-1}$ & $Y_{t}$ & $T_{t}=\frac{1}{3} Y_{t}$ & $D_{t}=A_{t}-T_{t}$ & $p_{t}=1-\frac{D_{t}}{x_{t}}$ & $\begin{array}{l}x_{t+1} \\
=-p_{t}+11\end{array}$ & $A_{t} Y_{t}$ \\
\hline 0 & 10 & 0 & 10 & 5 & 15 & 30 & 10 & 0 & 1 & 10 & 33,3 \\
1 & 10 & 2 & 12 & 3 & 15 & 30 & 10 & 2 & 0,8 & 10,2 & 40 \\
2 & 10,2 & 2 & 12,2 & 3 & 15 & 30,2 & 10,07 & 2,13 & 0,791 & 10,209 & 40,4 \\
3 & 10,209 & 2 & 12,209 & 3 & 15,1 & 30,309 & 10,103 & 2,106 & 0,794 & 10,206 & 40,28 \\
$\cdot$ & $\cdot$ & $\cdot$ & $\cdot$ & $\cdot$ & $\cdot$ & $\cdot$ & $\cdot$ & $\cdot$ & $\cdot$ & $\cdot$ & $\cdot$ \\
$\cdot$ & $\cdot$ & $\cdot$ & $\cdot$ & $\cdot$ & $\cdot$ & $\cdot$ & $\cdot$ & $\cdot$ & $\cdot$ & $\cdot$ & $\cdot$ \\
$\cdot$ & $\cdot$ & $\cdot$ & $\cdot$ & $\cdot$ & $\cdot$ & $\cdot$ & $\cdot$ & $\cdot$ & $\cdot$ & $\cdot$ & $\cdot$ \\
$\infty$ & 10,203 & 2 & 12,203 & 3 & 15,203 & 30,406 & 10,135 & 2,068 & 0,797 & 10,203 & 40,13 \\
\hline
\end{tabular}

b)

\begin{tabular}{|l|l|l|l|l|l|l|l|l|l|l|l|}
\hline 0 & 10 & 0 & 10 & 5 & 15 & 30 & 10 & 0 & 1 & 10 & $33, \overline{3}$ \\
1 & 10 & 2 & 12 & 3 & 15 & 30 & 10 & 2 & 0,8 & 10,2 & 40,0 \\
2 & 10,2 & 0 & 10,2 & 3 & 15 & 28,2 & 9,4 & 0,8 & 0,922 & 10,078 & 36,17 \\
3 & 10,078 & 0 & 10,078 & 3 & 14,1 & 27,178 & 9,059 & 1,019 & 0,899 & 10,101 & 37,08 \\
4 & 10,101 & 0 & 10,101 & 3 & 13,589 & 26,69 & 8,897 & 1,204 & 0,881 & 10,119 & 37,85 \\
5 & 10,119 & 0 & 10,119 & 3 & 13,345 & 26,464 & 8,821 & 1,298 & 0,872 & 10,128 & 38,24 \\
$\cdot$ & $\cdot$ & $\cdot$ & $\cdot$ & $\cdot$ & $\cdot$ & $\cdot$ & $\cdot$ & $\cdot$ & $\cdot$ & $\cdot$ & $\cdot$ \\
$\cdot$ & $\cdot$ & $\cdot$ & $\cdot$ & $\cdot$ & $\cdot$ & $\cdot$ & $\cdot$ & $\cdot$ & $\cdot$ & $\cdot$ & $\cdot$ \\
$\cdot$ & $\cdot$ & $\cdot$ & $\cdot$ & $\cdot$ & $\cdot$ & $\cdot$ & $\cdot$ & $\cdot$ & $\cdot$ & $\cdot$ & $\cdot$ \\
$\infty$ & 10,136 & 0 & 10,136 & 3 & 13,136 & 26,272 & 8,757 & 1,379 & 0,864 & 10,136 & 38,58 \\
\hline
\end{tabular}

Tabelle 6: Entwicklung des Bruttosozialprodukts und der Versorgung mit öffentlichen Gütern für $f=1$ 
a)

\begin{tabular}{|c|c|c|c|c|c|c|c|c|c|c|c|}
\hline$t$ & $k x_{t}$ & $b_{t}$ & $A_{t}=k x_{t}+b_{t}$ & $I_{t}$ & $c_{t}=\frac{1}{2} Y t-1$ & $Y_{t}$ & $T_{t}=\frac{1}{3} Y t$ & $D_{t}=A_{t}-T_{t}$ & $p_{t}=1-\frac{D_{t}}{x_{t}}$ & $\begin{array}{l}x_{t+1} \\
=-50 p_{t}+60\end{array}$ & $\frac{A_{t}}{Y_{t}}$ in \% \\
\hline 0 & 10 & 0 & 10 & 5 & 15 & 30 & 10 & 0 & 1 & 10 & $33, \overline{3}$ \\
\hline 1 & 10 & 2 & 12 & 3 & 15 & 30 & 10 & 2 & 0,8 & 20 & 40,0 \\
\hline 2 & 20 & 2 & 22 & 3 & 15 & 40 & $13, \overline{3}$ & $8, \overline{6}$ & $0,5 \overline{6}$ & $31, \overline{6}$ & 55 \\
\hline 3 & $31, \overline{6}$ & 2 & $33, \overline{6}$ & 3 & 20 & $56, \overline{6}$ & $18, \overline{8}$ & 14,7 & $0,5 \overline{3}$ & $33, \overline{3}$ & 59,41 \\
\hline 4 & $33, \overline{3}$ & 2 & $35, \overline{3}$ & 3 & $28, \overline{3}$ & $66, \overline{6}$ & $22, \overline{2}$ & $13, T$ & 0,61 & 29,5 & 53,0 \\
\hline 5 & 29,5 & 2 & 31,5 & 3 & $33, \overline{3}$ & 67,8 & 22,6 & 8,9 & 0,7 & 25 & 46,46 \\
\hline 6 & 25 & 2 & 27 & 3 & 33,9 & 63,9 & 21,3 & 5,7 & 0,772 & 21,4 & 42,25 \\
\hline 7 & 21,4 & 2 & 23,4 & 3 & 31,95 & 58,35 & 19,45 & 3,95 & 0,815 & 19,22 & 40,1 \\
\hline 8 & 19,22 & 2 & 21,22 & 3 & 21,18 & 53,4 & 17,8 & 3,42 & 0,822 & 18,9 & 39,74 \\
\hline 9 & 18,9 & 2 & 20,9 & 3 & 26,7 & 50,6 & 16,87 & 4,03 & 0,79 & 20,5 & 41,30 \\
\hline • & • & • & . & • & . & . & $\cdot$ & $\cdot$ & $\cdot$ & • & • \\
\hline • & . & . & · & . & • & - & • & - & - & • & • \\
\hline . & • & . & . & . & . & • & . & $\cdot$ & $\cdot$ & . & $\cdot$ \\
\hline$\infty$ & 23,87 & 2 & 25,87 & 3 & 28,87 & 57,74 & 19,25 & 6,62 & 0,73 & 23,87 & 41,34 \\
\hline
\end{tabular}

Tabelle 7 (Teil a): Entwicklung des Bruttosozialprodukts und der Versorgung mit öffentlichen Gütern für $f=50$ und Aufrechterhaltung des stabilitätspolitisch bedingten Defizits 
b)

\begin{tabular}{|c|c|c|c|c|c|c|c|c|c|c|c|}
\hline$t$ & $k x_{t}$ & $b_{t}$ & $A_{t}=k x_{t}+b_{t}$ & $I_{t}$ & $c_{t}=\frac{1}{2} Y_{t-1}$ & $Y_{t}$ & $T_{t}=\frac{1}{3} Y_{t}$ & $D_{t}=A_{t}-T_{t}$ & $p_{t}=1-\frac{D_{t}}{x_{t}}$ & $\begin{array}{l}x_{t+1} \\
=-50 p_{t}+60\end{array}$ & $\frac{A_{t}}{Y_{t}}$ in $\%$ \\
\hline 0 & 10 & 0 & 10 & 5 & 15 & 30 & 10 & 0 & 1 & 10 & $33, \overline{3}$ \\
\hline 1 & 10 & 2 & 12 & 3 & 15 & 30 & 10 & 2 & 0,8 & 20 & 40,0 \\
\hline 2 & 20 & 0 & 20 & 3 & 15 & 38 & $12, \overline{6}$ & $7, \overline{3}$ & 0,63 & 28,3 & 52,63 \\
\hline 3 & 28,3 & 0 & 28,3 & 3 & 19 & 50,3 & $16,7 \overline{6}$ & 11,53 & 0,59 & 30,37 & 56,26 \\
\hline 4 & 30,37 & 0 & 30,37 & 3 & 25,15 & 58,52 & 19,51 & 10,86 & 0,64 & 27,88 & 51,9 \\
\hline 5 & 27,88 & 0 & 27,88 & 3 & 29,26 & 60,14 & 20,05 & 7,83 & 0,719 & 24,04 & 46,36 \\
\hline 6 & 24,04 & 0 & 24,04 & 3 & 30,07 & 57,11 & 19,04 & 5 & 0,792 & 20,4 & 42,09 \\
\hline 7 & 20,4 & 0 & 20,4 & 3 & 28,56 & 51,96 & 17,32 & 3,08 & 0,849 & 17,55 & 39,26 \\
\hline 8 & 17,55 & 0 & 17,55 & 3 & 25,98 & 46,53 & 15,51 & 2,04 & 0,884 & 15,81 & 37,72 \\
\hline 9 & 15,81 & 0 & 15,81 & 3 & 23,27 & 42,08 & 14,03 & 1,78 & 0,887 & 15,63 & 37,57 \\
\hline 10 & 15,63 & 0 & 15,63 & 3 & 21,04 & 39,67 & 13,22 & 2,41 & 0,846 & 17,71 & 39,4 \\
\hline 11 & 17,71 & 0 & 17,71 & 3 & 19,84 & 40,55 & 13,52 & 4,19 & 0,763 & 21,83 & 43,67 \\
\hline 12 & 21,83 & 0 & 21,83 & 3 & 20,28 & 45,11 & 15,04 & 6,79 & 0,689 & 25,55 & 48,39 \\
\hline - & $\cdot$ & - & $\cdot$ & • & $\cdot$ & • & • & $\cdot$ & • & $\cdot$ & • \\
\hline - & - & · & - & · & - & $\cdot$ & • & - & • & • & • \\
\hline . & • & . & • & . & • & • & . & . & . & $\cdot$ & • \\
\hline$\infty$ & 22,153 & 0 & 22,153 & 3 & 25,153 & 50,306 & 16,769 & 5,384 & 0,757 & 22.153 & 44,04 \\
\hline
\end{tabular}

Tabelle 7 (Teil b): Entwicklung des Bruttosozialprodukts und der Versorgung mit öffentlichen Gütern für $f=50$ und Abbau des stabilitätspolitisch bedingten Defizits in Periode 2 
(IV.6e) $\quad x_{t+1}^{e}=-1000 p_{t}+1010$

zugrunde, was im Punkt $G(10,1)$ einer Steuerpreiselastizität $E=-100$ entspricht, so ergibt sich in der 6 . Periode eine Versorgung mit öffentlichen Gütern von $x_{6}=-142$ (vgl. Tabelle 8). Der Grund für dieses Ergebnis liegt darin, daß bei sehr elastischer Nachfrage eine geringe Preissenkung schon eine sehr hohe Nachfragesteigerung und damit eine sehr hohe Steigerung der Statsausgaben hervorruft. Diese Staatsausgaben erhöhen als Bestandteil der volks wirtschaftlichen Endnachfrage das Bruttosozialprodukt und damit das Steueraufkommen. Sobald die Staatsausgaben Konsumeffekte induzieren, steigen Bruttosozialprodukt und Steueraufkommen sehr stark an, was c.p. zu einem Abbau des Defizits führt. Aufgrund des starken Anstiegs der Steuereinnahmen wird bald ein Oberschuß erwirtschaftet, der wegen der hohen Steuerpreiselastizität der Nachfrage zu einer begativen Nachfrage nach dem öffentlichen Gut führt.

\section{Empirische Relevanz der Ergebnisse}

Mit derart hohen Steuerpreiselastizitäten ist in der Realität jedoch nicht zu rechnen ${ }^{1)}$. Schon der sich für $f_{d}=50$ im Punkt $G$ $(10,1)$ ergebende Elastizitätswert $E_{d}=-5$ ist, wenn man bisherigen empirischen Untersuchungen Glauben schenken darf, viel zu hoch. BERGSTROM und GOODMAN ${ }^{2)}$ haben für den kommunalen Bereich in den USA Steuerpreiselastizitäten zwischen - 0,01 und $-0,5$ bei allgemeinen Kommunalausgaben ${ }^{3}$ und $z$ wischen $-0,13$ und $-0,76$ bei den Ausgaben für Polizeischutz festgestellt. Bei den Ausgaben für Parks und Erholung schwanken die Elastizitäten zwischen 0 und $-0,68^{4)}$.

1) Wie bereits erwähnt ( $v g 1$. Fußnote 1, S. 75), ist auch nicht mit einem stabilitätspolitisch bedingten Defizit von $20 \%$ des Steueraufkommens zu rechnen. Infolgedessen wird auch die Senkung des Steuerpreises in der 1. Runde und die damit verbundene Nachfragesteigerung geringer ausfallen.

2) $\mathrm{Vgl}$. Bergstrom/Goodman (1973)

3) Ohne Bildungs- und Sozialausgaben.

4) Vgl. Bergstrom/Goodman (1973), S. $288 \mathrm{f}$. 


\begin{tabular}{|c|c|c|c|c|c|c|c|c|c|c|}
\hline$t$ & $k x_{t}$ & $b_{t}$ & $A_{t}=k x_{t}+b_{t}$ & $I_{t}$ & $c_{t}=\frac{1}{2} Y_{t-1}$ & $Y_{t}$ & $T_{t}=\frac{1}{3} Y_{t}$ & $D_{t}=A_{t}-T_{t}$ & $p_{t}=1-\frac{D_{t}}{x_{t}}$ & $x_{t+1}=-1000 p_{t}+1010$ \\
\hline 0 & 10 & 0 & 10 & 5 & 15 & 30 & 10 & 0 & 1 & 10 \\
1 & 10 & 2 & 12 & 3 & 15 & 30 & 10 & 2 & 0,8 & 210 \\
2 & 210 & 2 & 212 & 3 & 15 & 230 & 76,7 & 131,3 & 0,375 & 635 \\
3 & 635 & 2 & 637 & 3 & 115 & 755 & 251,7 & 385,3 & 0,393 & 617 \\
4 & 617 & 2 & 619 & 3 & 377,5 & 999,5 & 499,75 & 119,25 & 0,807 & 203 \\
5 & 203 & 2 & 205 & 3 & 499,75 & 707,75 & 235,9 & $-30,9$ & 1,152 & -142 \\
$\cdot$ & $\cdot$ & $\cdot$ & $\cdot$ & $\cdot$ & $\cdot$ & $\cdot$ & $\cdot$ & $\cdot$ & $\cdot$ & $\cdot$ \\
$\cdot$ & $\cdot$ & $\cdot$ & $\cdot$ & $\cdot$ & $\cdot$ & & $\cdot$ & & & $\cdot$ \\
\hline
\end{tabular}

Tabelle 8: Auswirkungen einer sehr hohen Steuerpreiselastizität 
POMMEREHNE und SCHNEIDER stellten für die Gesamtheit der 110 gröBten Schweizer Gemeinden Steuerpreiselastizitäten zwischen - 0,63 und $-0,66$ fest $^{1}$ ). BORCHERDING geht bei seiner Obersichtsarbeit über die Ursachen für das Wachstum der Staatsausgaben in den USA ${ }^{2}$ ) von einer Steuerpreiselastizität von $-0,5$ aus ${ }^{3}$ ).

Gehen wir in unserem Modell von einer Nachfragefunktion mit der (konstanten) Elastizität von $E_{f}=-0,5$ aus ${ }^{4}$, so ist das system zweifellos stabil. Der Staatsanteil steigt dann von $33,3 \%$ auf $34,64 \%$ im Falle der Aufrechterhaltung bzw. auf $39,16 \%$ im Falle des Abbaus des stabilitätspolitisch bedingten Defizits. Verwenden wir die Nachfragefunktion $x_{t+1}^{g}=10 / p_{t}\left(E_{g}=-1\right)$, so steigt der Staatsanteil von $33,3 \%$ auf $36,11 \%$ bzw. $40 \% 5)$. Der letzte Fall ist theoretisch besonders interessant, da sich die Höhe des Bruttosozialprodukts gegenüber dem Ausgangszustand nicht ändert, wohl aber seine Aufteilung zwischen Privatwirtschaft und Staat. Das stabilitätspolitisch bedingte Defizit führt in diesem Fall, obwohl es in der Folgeperiode wieder abgebaut wird, zu einem langfristigen Defizit in derselben Höhe, verbunden mit einer Preissenkung für öffentliche Güter und einer Ausdehnung des Staatsanteils.

\section{Folgerungen aus der Modellerweiterung}

Die Berücksichtigung des Einkommens-Konsum-Multiplikators und einkommensabhängiger Stevern führt bei Annahme realistischer GröBenordnungen der Steuerpreiselastizitäten hinsichtlich der zentralen Aus-

1) Vg1. Pommerehne/Schneider (1978), S. 392. Für die Gruppe der 27 repräsentativen Demokratien ohne Referendum wurden durch unterschiedliche Schätzgleichungen Steuerpreiselastizitäten zwischen - 0,28 und - 0,44 festgestelit. Vgl. ebenda, S. 397

2) $\mathrm{Vgl}$. Borcherding (1977a)

3) Vgl. ebenda, S. 49. Er verweist dabei u.a. auf Untersuchungen von Barr/Davis (1966) und Borcherding/Deacon (1972).

4) Wir verwenden dann die Nachfragefunktion $x_{t+1}^{f}=10 / \sqrt{p_{t}}$.

5) Der größere Staatsanteil im Falle des Abbaus des stabilitätspolitisch bedingten Defizits ist durch ein im Vergleich zur Beibehaltung desselben niedrigeres Sozialprodukt bedingt. 
sagen zu keinerlei Anderungen gegenüber dem Grundmode $11^{1}$ ). Im Unterschied zum Grundmodell ist es aber unerheblich, ob das stabilitätspolitisch bedingte Defizit wieder abgebaut wird oder nicht ${ }^{2}$. Die These von BW, die das Wachstum des Staatsanteils auf die durch Budgetdefizite bedingte Fiskalillusion und die damit verbundene Mehrnachfrage der Wähler nach öffentlichen Gütern zurückführt, kann daher nicht verworfen werden. Empirische Unterstützung erfährt die These durch eine Untersuchung NISKANENs ${ }^{3)}$, der für den Bereich der USA während des Zeitraums von 1947 - 1976 eine "perceived taxprice elasticity" 4 ) der Staatsausgaben von etwa - 0,6 feststellt. "The absolute magnitude of this partial effect, however, is not large ${ }^{5}$ )."

\section{Defizite und Statsanteil unter alternativen Annahmen}

Gehen wir von der alternativen Annahme aus, daB die Wähler die Höhe des Gesamtbudgets als unabhängig von ihrer individuellen Steuerschuld betrachten ${ }^{6)}$, so ist das vorliegende Modell nicht anwendbar, da die Wählerforderungen nach mehr öffentlichen Gütern nicht mehr von der Art der Finanzierung dieser Gijter abhängig sind. Vielmehr werden die Wähler demjenigen "politischen Unternehmer" ihre Stimme geben, der ihnen die meisten Begünstigungen verspricht. Der Politiker muB die Begünstigungen jedoch, wenn er gewählt wird und wiedergewählt werden will, bereitstellen und finanzieren. Stünde ihm die Verschuldungsalternative nicht zur Verfügung, so müBte er entweder die Steuern erhöhen oder er könnte nicht alle Wählerforderungen befriedigen.

1) Die instabilen Fälle setzen unrealistisch hohe Steuerpreiselastizitäten voraus. Auch be $i$ Beruicksichtigung einer hohen Konsumquote $(c=0,9)$ in Verbindung mit einem hohen Steuersatz $(\tau=0,5)$ bleibt das System im relevanten Elastizitätsbereich stabil.

2) Besonders Cansier (1977) weist auf den temporären Charakter fiskalpolitischer Maßnahmen hin.

3) Vgl. Niskanen (1978). Er weist jedoch selbst darauf hin, daB seine Ergebnisse "suggestive but not conclusive" sind (vgl. S. 597 und S. 602).

4) Ebenda, S. 595

5) Ebenda, S. 596

6) Vgl. Crain/Ekelund (1978), S. 818 
Obwohl a priori nicht gesagt werden kann, in welchem AusmaB die Politiker in der Vergangenheit bei Nichtvorhandensein der Verschuldungsalternative die Ausgaben gesenkt, die Steuern erhöht oder bestimmte Begünstigungen gar nicht versprochen hätten, so spricht doch einiges fur die These, daB das Ausgabenwachstum geringer gewesen wäre als das tatsächlich beobachtete. Geht man weiterhin davon aus, daB Beguinstigungen, nachdem sie einmal geschaffen wurden, nur schwer abzubauen sind, so wird ein weiterer EinfluB der Defizitfinanzierung auf das Wachstum des Statsanteils evident. Unter der plausiblen Annahme, daB Steuererhöhungen nur in begrenztem Ausmaß durchsetzbar sind, müBten nämlich bei Nichtvorhandensein der Verschuldungsalternative zur Schaffung neuer Beguinstigungen alte abgebaut werden, so da $B$ das Wachstum der Staatsausgaben geringer ausfallen würde. Selbst wenn man daher die These der Erhöhung des Staatsanteils aufgrund der Fiskalillusion nicht akzeptiert, so lassen sich dennoch gewichtige Argumente dafür finden, daß die Möglichkeit der Defizitfinanzierung das - möglicherweise primär durch andere Faktoren bedingte - Wachstum der Staatsausgaben und des Staatsanteils am Sozialprodukt zumindest beguinstigt hat.

\section{Fazit: Die empirische Relevanz der BUCHANAN/WAGNER-These}

Zusammenfassend läBt sich feststellen, $d a B$ die These von $B W$ einen Teil des in den letzten Jahren festgestellten kontinuierlichen Wachstums der Staatsausgaben und des Staatsanteils am Bruttosozialprodukt erklären kann. Zuzustimmen ist auch der These, daB die von den Autoren erklärten Effekte nicht aufgetreten wären, wenn das Budget über den Zyklus hinweg ausgeglichen gewesen wäre. Keinesfalls haben jedoch diese Effekte die GröBenordnung, die man aufgrund der Ausfuihrungen von $B W$ in einigen Publikationen ${ }^{1}$ erwarten würde. Auch können sie das schon lange vor KEYNES festgestellte relative Wachstum des Staatsanteils nicht erklären ${ }^{2)}$. Die These

1) Vgl. Z.B. Buchanan/Wagner (1978c), S. $79 \mathrm{f}$.

2) So hat Adolph Wagner schon im vorigen Jahrhundert, also in einer Zeit, als an eine bewuBte Konjunktursteuerung noch nicht $z u$ denken war, se in "Gesetz der zunehmenden Staatstätigkeit" entwickelt. Vgl. Wagner, A. (1892), S. $892 \mathrm{ff}$. Vgl. auch Gordon, D.F. (1978), S. 584 
der Autoren, die durch "keynesianische" Budgetdefizite hervorgerufene Fiskalillusion führe zu einer Erhöhung des Staatsanteils am Sozialprodukt, muB daher als eine Erklärungsmöglichkeit für das Wachstum der Staatsausgaben und des Staatsanteils am Bruttosozialprodukt vor allem seit Anfang der Sechziger Jahre angesehen werden. In ihrer Stellungnahme zur Kritik an ihrer Publikation räumen die Autoren selbst diesen Sachverhalt ausdrücklich ein ${ }^{1}$.

Somit ist dem Einwand BRUNNERs, obwohl er theoretisch unter bestimmten Annahmen widerlegt werden kann, im Ergebnis zuzustimmen. Das seit über einem Jahrhundert beobachtete kontinuierliche Wachstum der Staatsausgaben und des Staatsanteils am Bruttosozialprodukt kann durch die von BW aufgezeigten Effekte nicht insgesamt erklärt werden.

Es ist allerdings denkbar und wahrscheinlich, daB die Möglichkeit der Defizitfinanzierung das durch andere Faktoren bedingte Wachstum der Staatsausgaben erleichtert hat. Angesichts der im Rahmen der Konsolidierungsbemühungen des Bundeshaushalts in den Jahren 1980 und 1981 zu Tage getretenen Schwierigkeiten beim Abbau von Begünstigungen spezieller Gruppen ist erneut offensichtlich geworden, daB die Verschuldungsmöglichkeit den um Wählerstimmen konkurrierenden Politikern sehr entgegenkommt. Gäbe es die Verschuldungsalternative nicht, so müBten entweder Vergünstigungen abgebaut oder neue Belastungen in Form von Steuererhöhungen eingeführt werden. Die These, daB das Wachstum der Staatsausgaben bei Vorliegen der Regel des Budgetausgleichs zwar nicht unterblieben, aber geringer ausgefallen wäre, kann daher mit hoher Wahrscheinlichkeit als bestätigt gelten.

1) "We make no claim that the Keynesian bias is the only significant explanatory factor. Our purpose was to isolate this factor . . " Buchanan/Wagner (1978), S. 634. Buchanan selbst nennt an anderer Stelle weitere Ursachen für das beobachtete Phänomen. Vgl. Buchanan (1977). Weitere Oberblicksdarstellungen finden sich bei Borcherding (1977a) sowie bei Amacher/Tollison/Willet (1975). 
Im Rahmen dieses Kapitels soll die neoinstitutionalistische These untersucht werden, staatliche Budgetdefizite führten zu einer Erhöhung der Geldmenge und damit zur Inflation, auch wenn sich die Regierung nicht direkt bei der de jure unabhängigen Zentralbank verschulde. Diese These ist insbesondere deshalb zu diskutieren, weil in der Bundesrepublik Deutschland eine Verschuldung des Staates bei der Zentralbank nahezu ausgeschlossen ist ${ }^{1)}$. Sollten daher die von BW postulierten Inflationswirkungen statlicher Budgetdefizite auch für die Bundesrepublik Deutschland zutreffen, so ist $z u$ belegen, daß die zwar nach dem Gesetz unabhängige Zentralbank ${ }^{2}$ ) auf staatliche Budgetdefizite aber typischerweise mit einer Erhöhung der Geldmenge reagiert.

\section{Die neoinstitutionalistische Argumentation}

BW nehmen eine nach dem Gesetz unabhängige Zentralbank an, die in der Lage ist, das Geldangebot zu steuern und deren primäre Aufgabe in der Sicherung der Preisniveaustabilität besteht ${ }^{3)}$. Welche Reaktion ist von dieser Institution zu erwarten, wenn das statiche Budget ein Defizit aufweist, das der staat durch Verschuldung im privaten Sektor finanziert?

Das staatliche Wertpapierangebot senkt nach Ansicht von BW den Kurs dieser Papiere und bewirkt damit eine Zinssteigerung auf dem Kapi-

1) Nach $\S 20$ Bundesbankgesetz ist die Bundesbank berechtigt (nicht verpflichtet), dem Bund, den Ländern sowie einzelnen Sondervermögen kurzfristige Kassenkredite zu gewähren, die für den Bund 6 Mrd. DM nicht überschreiten dürfen. Vgl. Deutsche Bundesbank (1980), S. 32. Allerdings steht dem Bund nach $\S 27$ Bundesbankgesetz der Großteil des Bundesbankgewinns zu. Im AusmaB des vom Bund in Anspruch genommenen Gewinnanteils erfolgt somit eine Geldschöpfungsfinanzierung von Staatsausgaben, obwohl der Bundeshaushalt formal ke in Defizit aufweist. Ein Inflationsimpuls resultiert allerdings nur, wenn die Bundesbank aufgrund einer Gewinnabführung ihr Geldmengenziel ändert.

2) Vgl. v. Spindler/Becker/Starke (1973), S. 263

3) Vgl. Buchanan/Wagner (1977), S. $112 \mathrm{f}$. 
talmarkt. Bleibt die Zentralbank passiv, so bewirkt dies einen Rückgang der privaten Investitionen, eine Allokationsverzerrung zugunsten des privaten Konsums und eine - ebenfalls allokativ ineffiziente - Ausdehnung des Staatsanteils ${ }^{1)}$. Die mit dem Rückgang der privaten Investitionen verbundene Abnahme der Beschäftigung wirkt sich negativ auf die Popularität der Regierung aus, die folglich an einer Erhöhung der Geldmenge interessiert ist, welche die Anspannung auf dem Kapitalmarkt beseitigen würde. Eine solche MaBnahme wird von der Regierung insbesondere deshalb befürwortet, weil für eine möglicherweise resultierende Erhöhung der Inflationsrate die Zentralbank, nicht jedoch die Regierung, direkt verantwortlich gemacht wird ${ }^{2)}$. In dieser situation kommt es daher zu einem Konflikt der Interessen der auf monetäre Expansion drängenden Regierung und der am Ziel der Preisniveaustabilität orientierten Zentralbank.

Das Verhalten der verantwortichen Entscheidungsträger der Zentralbank in dieser Konfliktsituation kann durch die Annahmen der Birokratietheorie erklärt werden ${ }^{3)}$. Die Zentralbankbürokraten sind danach vor allem an persönlichem Prestige und Erhaltung ihrer (unabhängigen) Stellung interessiert ${ }^{4}$ ).

Zunächst sind die Auswirkungen einer konsequent am Ziel der Preisniveaustabilität orientierten Geldpolitik auf das Prestige der Zentralbankbürokraten zu beachten. In einer Phase hoher Zinssätze, sinkender Investitionen und steigender Arbeitslosigkeit wird die Zentralbank die Geldmenge nach Ansicht von BW vor allem deshalb erhöhen, weil sie sonst von der offentlichkeit und der Presse für

1) Vgl. Buchanan/Wagner (1977), S. 113

2) $\mathrm{Vgl}$. ebenda, S. $110 \mathrm{f}$.

3) Vg1. auch den Verwe is von Buchanan/Wagner (1977), S. 119, Fußnote 8.

4) Vgl. zum Verhalten von Zentralbankbürokraten vor allem Chant/Acheson (1972), S. $14 \mathrm{f}$. und Acheson /Chant (1973), S. $638 \mathrm{ff}$., aber auch Downs (1967), S. 2 und Frey/Schneider (1978b), S. 3 f. 
diese negativen Effekte verantwortich gemacht wird ${ }^{1)}$. Diese Reaktion ist selbst dann zu erwarten, wenn sich die Zentralbankbürokraten über die sozialen Nutzen einer an sich erforderlichen restriktiven Politik im klaren sind. Entscheidend ist, daB diese sozialen Nutzen nicht in individuelle Nutzen der monetären Entscheidungsträger transformiert werden ${ }^{2)}$. Hinzu kommt, daß bei einer starken Beeinträchtigung des Beschäftigungsziels auch die unabhängige StezZung der Zentralbank bedroht sein könnte, da die offentlichkeit in diesem Fall einer möglichen Einschränkung der Notenbankautonomie weniger Widerstand entgegensetzen würde. "In the long run the government has the means to overrule the central bank, these ranging from dismissing the central bank's president and members of the governing board and replacing them with more compliant people, to the threat of completely taking over its functions 3 )."

Da somit eine Politik des "easy money" auch für die Zentralbankbuirokraten "easy" ist, eine Politik des "tight money" ihnen jedoch primär schadet, werden die Verantwortlichen der Zentralbank auf ein Budgetdefizit mit einer Geldmengenerhöhung reagieren ${ }^{4)}$.

\section{Kritik der neoinstitutionalistischen Argumentation}

Die Kritik der neoinstitutionalistischen Argumentation befaBt sich zunächst mit der Crowding-out-These der Autoren und fragt dann unter der Annahme, $d a B$ aufgrund der Kreditmarktverschuldung des Staates tatsächlich mit Verdrängungseffekten gerechnet werden muß, nach den Reaktionen der Zentralbank auf die angenommene Entwicklung. Mit einer Erhöhung der Geldmenge ist nämlich nur dann zu rechnen, wenn

1) Vgl. Buchanan/Wagner (1977), S. 118. Vgl. auch Poole (1979), S. 236, der von einer "public relations position" der Zentralbank spricht.

2) Vgl. zu diesem Phänomen auch Tullock (1971), S. 915 ff.

3) Frey/Schneider (1978b), S. 4. Vgl. auch Buchanan/Wagner (1977), S. 118 f. und Poullain (1973), S. 43

4) Vgl. Buchanan/Wagner (1977), S. $118 \mathrm{f}$. Das Verhalten des Federal Reserve System in der zweiten Hälfte des Jahres 1981 spricht indessen gegen diese These, da trotz hoher Zinsen und hoher Arbeitslosigkeit eine Politik des "tight money" betrieben wurde. 
die Zentralbank an einem niedrigen Zinsniveau interessiert ist bzw. zur Stabilisierung des Zinsniveaus gezwungen werden kann ${ }^{1}$. In diesem Zusammenhang spielt die Analyse der De-facto-Abhängigkeit der Zentralbank vom Staat eine zentrale Rolle. Da im Rahmen dieser Arbeit auch die Obertragbarkeit der Ergebnisse von BW auf die Verhältnisse in der Bundesrepublik Deutschland überprüft werden soll, steht die Untersuchung der Zentralbankautonomie in der Bundesrepublik Deutschland und in den USA im Zentrum der Untersuchung ${ }^{2}$. AnschlieBend werden die Inflationswirkungen staticher Budgetdefizite diskutiert, wobei die Analyse der Geldmengenwirkungen von Budgetsalden in den USA und in der Bundesrepublik Deutschland im Vordergrund stehen.

\subsection{Das Argument eines zinsbedingten crowding-out}

Die Argumentation von BW hebt zunächst auf eine durch die statliche Kreditnachfrage bedingte Zinssteigerung ab, die die privaten Investitionen zurückdrängt und dadurch die Beschäftigungsprobleme verschärft. In diesem Zusammenhang kann an die Ausführungen über Crowding-out-Effekte aus dem dritten Kapitel angeknüpft werden ${ }^{3)}$. Entsprechend der Argumentation von BW, die auf das kurzfristige wahltaktische Kalkuil demokratisch gewählter Politiker abhebt, wird das Hauptgewicht auf die Analyse kurz- und mittelfristig auftretender Crowding-out-Effekte gelegt ${ }^{4}$ ). Damit kommt aber der aktuellen konjunkturellen Situation, in der der Staat Defizite macht, entscheidende Bedeutung zu.

1) Vgl. Neumann (1981), S. 125 und Hein (1981), S. 4

2) Einen kurzen Oberblick über das Verhältnis von Regierung und Zentralbank in anderen Staaten gibt Lipfert (1974), S. $139 \mathrm{ff}$. Vgl. auch zum Verhältnis von Zentralbank und Staat in Großbritannien Lipp (1978), S. 362 ff. und Searjant (1976).

3) $\mathrm{Vgl}$. Abschnitt III.1.4.8., S. $46 \mathrm{ff}$.

4) Im langfristigen Vollbeschäftigungsgleichgewicht hat ein staatliches Budgetdefizit immer eine Verminderung der Kapitalintensität und/oder eine Erhöhung der Inflationsrate zur Folge. Eine Ausnahme bildet der Fall einer gleichzeitigen Senkung des Anteils der Staatsausgaben am Sozialprodukt, mit der aber realistischerweise nicht gerechnet werden kann. Vgl. Feldstein (1980), S. 640 
Während in rezessiven Phasen aufgrund der geringen privaten Kreditnachfrage und eines vergleichsweise elastischen Kreditangebots ${ }^{1}$ ) noch in größerem Umfang Kredite aufgenommen werden können, ohne daB Zinssteigerungen auf dem Kapitalmarkt befürchtet werden müssen, können sich Probleme ergeben, wenn der Staat den Kapitalmarkt auch noch im beginnenden Aufschwung in Anspruch nimmt. In dieser Situation trifft die kreditnachfrage des Staates mit der durch die staatliche Nachfragestimulierung induzierten verstärkten privaten Kreditnachfrage zusammen, was zu Zinssteigerungen führen wird. Verhindert die Zentralbank einen zinsinduzierten Kapitalimport und sind die privaten Ausgaben zinselastisch, so kann der gerade in Gang gesetzte Wiederaufschwung gefährdet werden ${ }^{2}$. Dies ist insbesondere dann der Fall, wenn sich der staat in dieser Situation aus nichtkonjunkturellen Gründen verschuldet ${ }^{3)}$, oder wenn die Defizite aus zurückliegenden fiskalpolitischen Maßnahmen noch nicht völlig abgebaut sind. Letztere leisten dann zwar nur noch einen geringfügigen Beitrag zur Oberwindung der Rezession, rufen aber eine zusätzliche Anspannung auf dem Kapitalmarkt hervor ${ }^{4}$.

Diese These einer Verdrängung privater Investitionen auf dem Kapitalmarkt wurde vor allem von STOTZEL ${ }^{5}$ ) angegriffen. Seiner Ansicht nach werden durch die Kreditnachfrage des Staates "die Möglichkeiten privater Kreditaufnahme... per Saldo nicht im mindesten geschmälert"6). Der Grund liege darin, daß der Staat die aufgenommenen Gelder sofort wieder ausgebe und dadurch auf dem Kapitalmarkt

1) Vgl. Gandenberger (1980), S. $17 \mathrm{ff}$.

2) Vgl. Ehrlicher (1975), S. 453

3) Man spricht in diesem Zusammenhang von "strukturellen" Budgetdefiziten, vgl. Ehrlicher (1975), S. 449 f., Ehrlicher (1979), S. 395 und Dieckheuer (1977), S. 553, die auch auf die unterschiedliche Abgrenzung des strukturellen Defizits durch den Wissenschaftlichen Beirat (1975) und den Sachverständigenrat (1975), Tz. 424 eingehen.

4) Vg1. Dieckheuer (1978), S. 23

5) Vgl. Stützel (1978), S. 444 ff., vgl. auch Nölling (1979), S. 486 und Teschner (1980), S. 121

6) Stützel (1978), S. 446 
ein zusätzliches Kapitalangebot schaffe ${ }^{1}$ ). Dieser Aspekt werde von den Vertretern der Crowding-out-These vernachlässigt. Der Kapitalmarkt sei keine "Quelle, die eine bestimmte, unabhängig festliegende jährliche Schüttung an liquiden Mitteln"2) bringe, "die Ausgabenwirtschaft des Staates" sei "eher mit einer Fontäne vergleichbar, die mitten im Teiche steht: Unten saugen die Pumpen die liquiden Mittel vom Teich ab, oben werden sie als Springbrunnen über die ganze Bevölkerung verteilt, fallen also damit wieder in den Teich zurück" 3 ).

Das Argument STOTZELs 1 äßt sich am einfachsten anhand eines Modells einer geschlossenen Volkswirtschaft diskutieren, die in den privaten und den öffentlichen Sektor disaggregiert wird ${ }^{4}$. Der private Sektor umfaBt dabei neben den privaten Haushalten und Unternehmen auch das private Bankensystem, der öffentliche Sektor wird in die Gebietskörperschaften und die Zentralbank unterteilt.

Das Nettovermögen des privaten Sektors setzt sich in diesem Modell aus dem zu Marktpreisen bewerteten Bestand an Sachvermögen sowie den Forderungen gegen die Gebietskörperschaften und die Zentralbank zusammen. Daher wird sich ein Ausgabenüberschuß des States, der zu einer Kreditnachfrage auf dem Kapitalmarkt führt, bei Passivität der Zentralbank zwangsläufig in einer Erhöhung der Forderungsposition des privaten Sektors niederschlagen. Da der Staat somit in derselben Höhe, in der er Schulden macht, zusätzliches privates Finanzvermögen schafft, kann es zu keinen Mengenproblemen auf den kreditmärkten kommen ${ }^{5}$ ).
1) Vg1. hierzu auch Eisner (1977), S. 112
2) Stuitzel (1978), S. 445
3) Ebenda, S. 446
4) Vgl. auch Duwendag, u.a. (1977), S. $323 \mathrm{f}$.
5) Vgl. ebenda, S. 323 
Dieser rein kreislaufmäßige Zusammenhang gilt jedoch nur ex post ${ }^{1)}$, d.h. nach AbschluB aller durch die Staatsverschuldung hervorgerufenen Anpassungsprozesse. Zu klären bleibt die Frage, ob und wie es dem Staat gelingt, eine erhöhte Verschuldung durchzusetzen. Dabe $i$ ist insbesondere zu untersuchen, welche Anpassungsvorgänge im privaten Sektor zu dem oben definierten Ex-post-Gleichgewicht führen.

Der Prozess kann systematisch wie folgt beschrieben werden: Befinden sich die privaten Wirtschaftssubjekte vor der beabsichtigten Wertpapieremission des Staates in einem Portfolio-Gleichgewicht, so werden sie das statiche Wertpapierangebot nur dann in ihr Portefeuille aufnehmen, wenn ihnen die Staatspapiere gegenüber den anderen Anlagealternativen eine ausreichende Rendite versprechen. Der Staat muß also seine Wertpapiere mit einer attraktiven Nominalverzinsung ausstatten, die das Portfolio-Gleichgewicht der Privaten stört und sie zu einer Substitution anderer Aktiva durch statiche Wertpapiere veranlaBt ${ }^{2}$ ).

Die weiteren Reaktionen hängen nun von der relativen Intensität der Substitutionsbeziehungen zwischen den unterschiedlichen Aktiva im Portefeuille der Wirtschaftssubjekte $a b^{3}$ ). Die Zinssätze derjenigen Aktiva, zu denen die emittierten Statsschuldtitel relativ gute Substitute sind, werden in Relation zu den Zinssätzen der übrigen Aktiva steigen ${ }^{4}$ ).

Verschuldet sich der Staat also langfristig, so ist anzunehmen, da $\beta$ das Zinsniveau für langfristige Wertpapiere auf dem Kapitalmarkt steigt. Da gemeinhin angenommen wird, daß das Investitionsniveau negativ mit dem langfristigen Kapitalmarktzins korreliert ist ${ }^{5}$,

1) Vgl. Duwendag, U.a. (1977), S. 324 und Francke (1979), S. 367

2) Vgl. auch Willms (1978), S. 442

3) Vgl. Francke (1979), S. 336

4) Vgl. Tobin (1961), S. 34

5) Vgl. Z.B. Hendershott (1976), S. 1185 
ist im Aufschwung mit Crowding-out-Effekten zu rechnen, deren AusmaB von der Intensität der Substitutionsbeziehungen der staatlichen Wertpapiere und der übrigen Aktiva abhängt. M.a.W. sind staatliche Wertpapiere und Realvermögen bessere Substitute als statliche Wertpapiere und Geld, so stellt sich ein kontraktiver Effekt ein, der zu einem partiellen crowding-out ${ }^{1)}$ privater Investitionen durch die staatliche Kreditaufnahme führt ${ }^{2}$. Ein vollständiges crowding-out ist jedoch nur dann zu erwarten, wenn staatliche Wertpapiere und Realkapital vollkommene Substitute sind. Verschuldet sich der staat dagegen kurzfristig, so wird ein crowding-out weniger wahrscheinlich, da Geld und kurzfristige Wertpapiere bessere Substitute als Realkapital und kurzfristige Wertpapiere sind.

Als Fazit bleibt somit festzuhalten, daß mit einem Verdrängen privater Nachfrage vor allem dann zu rechnen ist, wenn der staat im Aufschwung als Anbieter langfristiger Wertpapiere auftritt und wenn langfristige Wertpapiere des Staates als relativ gute Substitute zu Realkapital angesehen werden. Gerade die Erfüllung der letzten Bedingung ist jedoch keineswegs gesichert. Mittelfristig kann ein Budgetdefizit sogar eine Erhöhung des Kreditangebots bzw. eine Verminderung der Kreditnachfrage bewirken, da über die von niemand ernsthaft bestrittene expansive Initialwirkung des staatlichen deficit-spending eine erhöhte Ersparnis der Haushalte und/

1) Vgl. zum Begriff des partiellen und des vollständigen crowding-out Rose (1980), S. $357 \mathrm{f}$.

2) Vgl. Francke (1979), S. 366 sowie Besters (1980), S. 421 f. Lediglich Tobin (1963) geht be i der betrachteten Maßnahme von einem expansiven Effekt aus. Se in Ansatz hebt auf das Verhältnis zwischen Grenzproduktivität des Kapitals und erforderlicher Mindestverzinsung des Kapitals (supply-price of capital $=S P C$ ) $a b$. Der SPC wird allerdings nicht nur durch relative Ertragsratendifferenzen (vgl. hierzu Tobin (1961), S. $34 \mathrm{ff}$. ), sondern auch durch das Risiko alternativer Anlageformen beeinflußt. Letzteres hängt vom Verhältnis des Realvermögens zum monetären Vermögen $a b$ und steigt, wenn der Bestand einer Vermögensart gegenüber der anderen zunimmt. Im dargestellten Fall steigt demnach das Risiko aufgrund einer Zunahme des monetären Vermögens, was die Nachfrage nach Realvermögen verstärkt und somit c.p. einen expansiven Effekt bedeutet. Dieser expansive Effekt überwiegt den kontraktiv wirkenden Effekt der Ertragsratendifferenzen, so daß sich ein expansiver Gesamteffekt ergibt. 
oder verbesserte Selbstfinanzierungsmöglichkeiten der Unternehmen induziert werden ${ }^{1}$ ).

Wenngleich die empirischen Beiträge zur Crowding-out-Kontroverse keine eindeutigen Schlußfolgerungen zulassen, so ist doch der Argumentation STOTZELs, die von einer kurzfristigen Finanzierung staatlicher Defizite ausgeht ${ }^{2}$, im Ergebnis tendenziell zuzustimmen ${ }^{3)}$, obwohl sie zu stark auf kreislaufzusammenhänge abhebt und die preistheoretisch zu erklärenden Vermögensdispositionen privater Anlager vernachlässigt ${ }^{4}$ ).

Die neoinstitutionalistische These eines zinsbedingten crowdingout ist somit nicht zwingend, kann aber auch nicht eindeutig widerlegt werden. Da die weitere Argumentation von BW auf der Crowdingout-These aufbaut, wird im folgenden zunächst unterstellt, daB be $i$ einer Verschuldung des Staates im privaten Sektor mit Verdrängungseffekten gerechnet werden muß. Unter dieser Annahme wird untersucht, ob sich die Zentralbank der Forderung der Regierung nach einer - möglicherweise inflatorisch wirkenden - Geldmengenerhöhung erfolgreich widersetzen kann ${ }^{5}$ ). In diesem Zusammenhang ist auch auf eine in der Literatur oft erwähnte und seit 1977 gesetzlich fixierte Verpflichtung der amerikanischen Zentralbank zur Stabilisierung des Zinsniveaus einzugehen, wenngleich letztere nicht notwen-

1) Vgl. auch Hendershott (1976), S. 1185 und S. $1195 \mathrm{ff}$. und Meyer (1980), S. 30, die auf die Möglichkeiten eines "pulling-in" privater Investitionen hinweisen. Dabei wird eine Abhängigkeit der privaten Investitionen vom Volkseinkommen unterstellt.

2) Vgl. Stuitzel (1978), S. 447

3) Vg1. Dieckheuer (1980), S. 141 sowie Rürup (1980), S. 425 f., der die Fontänentheorie Stuitzels in der gegenwärtigen Situation für "we it relevanter" (S. 426) hält als die Quellentheorie, obwohl auch er auf die nicht hinreichend berücksichtigten Verhaltensweisen der Kapitalanbieter und -nachfrager hinweist.

4) Vgl. auch Francke (1979), S. $367 \mathrm{f}$.

5) Da die Analyse auf das Zentralbankverhalten abhebt, wird unterstellt, daB die Regierung in der betrachteten Situation eine Erhöhung der Geldmenge befürwortet, obwohl ihr die damit verbundene Erhöhung der Inflationsrate Stimmenverluste einbringen kann. ( $\mathrm{Vgl}$. die in III.2. genannten politometrischen Untersuchungen.) 
dig auf die Befürchtung von Crowding-out-Effekten zurückgeführt werden muB.

\subsection{Die Reaktion der Zentralbank auf ein steigendes Zinsniveau. Eine Analyse der Geldmengen- und Inflationswirkungen staat- licher Budgetdefizite}

BW heben in ihrer weiteren Argumentation auf die Bedrohung der unabhängigen Stellung der Zentralbank und auf das Prestige der Zentralbankbürokraten ab. Weiterhin verweisen sie auf die empirische Entwicklung der Geldmenge und der Budgetdefizite in den USA, welche ihrer Ansicht nach die These der De-facto-Abhängigkeit der Zentralbank von der Regierung stüzt ${ }^{1}$ ).

Im folgenden wird zunächst untersucht, ob aufgrund der institutionellen Bedingungen, innerhalb derer Zentralbankpolitik in der Bundesrepublik Deutschland und in den USA betrieben wird, mit der Defacto-Abhängigkeit der Deutschen Bundesbank bzw. des Federal Reserve system gerechnet werden muß. Im AnschluB daran erfolgt eine empirische Analyse der Bundesbankautonomie. AbschlieBend wird die These der Geldmengen- und Inflationswirkungen staatlicher Budgetdefizite anhand ökonometrischer Untersuchungen überprüf.

2.2.1. Institutionelle Analyse der Zentralbankautonomie in der Bundesrepublik Deutschland und in den USA

Die Analyse der Unabhängigkeit der Deutschen Bundesbank und des Federal Reserve System befaßt sich vor allem mit der personellen Unabhängigkeit der zentralen Entscheidungsträger dieser Institutionen. In der Bundesrepublik Deutschland werden zusätzlich die Auswirkungen der Koordinierungsverflechtung zwischen Bundesbank und Staat auf die Unabhängigkeit der Bundesbank untersucht. In den USA spielt

1) Vgl. Buchanan/Wagner (1977), S. $115 \mathrm{ff}$. 
weiterhin die Frage der Unabhängigkeit der Zentralbank als Institution eine Rolle ${ }^{1)}$.

\subsubsection{Die personelle Unabhängigkeit der Deutschen Bundesbank}

Da die rechtlich unabhängige Stellung einmal berufener Zentralbankmitglieder gesichert ist ${ }^{2)}$, muB eine Analyse der personellen Unabhängigkeit der Entscheidungsträger der Notenbank vor allem auf die Einflußmöglichkeiten der Regierung bei der Besetzung der zentralen WizZensbizdungsorgane der Bundesbank abstellen. Oberstes Entscheidungsorgan und damit zentrales Untersuchungsobjekt einer Analyse der Möglichkeiten regierungspolitischer Beeinflussung von Bundesbankentscheidungen ist der Zentralbankrat, der sich aus dem Präsidenten und Vizepräsidenten der Deutschen Bundesbank, den (bis zu acht) weiteren Mitgliedern des Direktoriums und den elf Präsidenten der Landeszentralbanken zusammensetzt ${ }^{3}$ ).

Präsident und Vizepräsident der Bundesbank werden - ebenso wie die weiteren Mitglieder des Direktoriums - vom Bundespräsidenten auf Vorschlag der Bundesregierung bestellt $t^{4}$, was auf eine gewisse $A b-$ hängigkeit von der Regierung schließen lassen könnte. Gegen eine Abhängigkeit spricht jedoch die achtjährige Amtsperiode der Direktoriumsmitglieder, die zwei oder gar drei Legislaturperioden tangiert. Probleme für die persönliche Abhängigkeit einzelner Direktoriumsmitglieder können sich allenfalls aus der Möglichkeit ihrer Wiederwahl ergeben ${ }^{5}$ ). Letztere bringt einzelne Mitglieder, deren

1) Auch in der Bundesrepublik Deutschland ist die Deutsche Bundesbank als Institution verfassungsmäBig nicht geschuitzt. Vgl. v. Spindler/Becker/Starke (1973), S. 171

2) Vgl. Huppertz (1977), S. 74. Als Abberufungsgründe kommen lediglich grobe Pflichtverletzung oder Unfähigkeit zur ordnungsmäßigen Geschäftsführung in Betracht. Vgl. v. Spindler/Becker/Starke (1973), S. 231

3) Vgl. Jarchow (1974), S. 57 ff. sowie Deutsche Bundesbank (1980), S. 7

4) Der Zentralbankrat hat lediglich ein Anhörungsrecht bei der Bestellung von Zentralbankratsmitgliedern. Er kann jedoch die Berufung für ihn nicht akzeptabler Mitglieder nicht verhindern. Nicht einmal eine eventuelle Nichtbeachtung des Anhörungsrechts seitens der Regierung macht eine bereits vollzogene Ernennung unwirksam. Vgl. v. Spindler/Becker/Starke (1973), S. 230

5) Vgl. Kaiser (1980), S. 38 f. 
Amtszeit sich ihrem Ende zuneigt, in eine Abhängigkeitsposition gegenüber der Regierung als Vorschlagsinstanz und könnte daher eine Einflußnahme der Regierung auf das Abstimmungsverhalten einzelner Direktoren ermöglichen. In der Tat hat die Vergangenheit gezeigt, daß bei der Neubesetzung von Direktoriumsämtern versucht wird, politisch genehme Kandidaten ins Amt zu bringen ${ }^{1}$ ).

Eine allzu starke Beeinflussung aktueller Zentralbankentscheidungen auf dem Wege der Neu-bzw. Wiederbesetzung von Direktoriumsämtern ist jedoch nicht zu erwarten, da eine Neu-oder Wiederbesetzung i.d.R. nicht gerade dann anstehen wird, wenn Regierung und Zentralbank im Konflikt stehen. Weiterhin wird das Direktorium nicht zu einem einzigen Zeitpunkt insgesamt neu besetzt, so daB ein EinfluB der Regierung auf das Abstimmungsverhalten des Gremiums im konkreten Einzelfall weniger wahrscheinlich ist.

Zudem ist anzunehmen, daB die Zentralbankbuirokraten aufgrund der neuen Aufgaben, die sie nach ihrer Berufung unabhängig zu verfolgen verpflichtet sind, und aufgrund des Prestiges, das sie bei erfolgreicher Erfüllung dieser Aufgaben genieBen, ein eigenes Rollenverständnis entwickeln, das stärker ist als die parteipolitische Adhäsion zu den Politikern, denen sie ihre Ernennung verdan$k^{2}{ }^{2}$. Diese These wird beispielsweise durch das Verhalten der Zentralbank Ende 1980/Anfang 1981 gestützt. Obwohl vor der Besetzung des Präsidentenamtes durch Karl-0tto Pöhl erwartet worden war, daB sich der neue Bundesbankpräsident aufgrund seiner Mitgliedschaft in der SPD eher für eine die Beschäftigungspolitik stützende denn für eine Hochzinspolitik einsetzen würde, hielt die Deutsche Bundesbank Ende 1980 trotz scharfer Kritik seitens der Politiker und der führenden Wirtschaftsforschungsinstitute an ih-

1) Vgl. Huppertz (1977), S. $80 \mathrm{ff}$.

2) Vgl. ebenda, S. 82. Im übrigen ist darauf hinzuweisen, daB die Mehrzahl der Direktoriumsmitglieder eine oft mehr als zwanzigjährige Notenbanklaufbahn hinter sich hat (vgl. Kaiser (1980), S. 33 f.) und sich somit stark mit den eigenständigen Notenbankzielen identifizieren wird. 
rer außenwirtschaftlich motivierten Hochzinspolitik fest. Die Kontroverse des ehemaligen Bundesbankpräsidenten Klasen mit dem damaligen Wirtschaftsminister Schiller zeigt weiterhin, daß parteipolitisch ernannte Zentralbankmitglieder, nachdem sie einmal in ihre unabhängige Stellung berufen worden sind, durchaus die Fähigkeit entwickeln, ihrerseits die Regierung von der Notwendigkeit der Implementierung der wirtschaftspolitischen Vorstellungen der Notenbank zu überzeugen ${ }^{1}$ ). Es scheint somit berechtigt, von der personellen Unabhängigkeit der Direktoriumsmitglieder auszugehen.

Die neben den Direktoriumsmitgliedern im Zentralbankrat vertretenen Präsidenten der Landeszentralbanken werden de facto von der jewe $i l i g e n$ Landesregierung bestimmt ${ }^{2}$, also in der Regel auch von der Partei, die sich auf Bundesebene in der Opposition befindet. Dieses föderative Element vermindert die EinfluBmöglichkeiten der Bundesregierung auf die Zentralbank, zumal die von der Bundesregierung bestellten Mitglieder des Zentralbankrates rein rechnerisch in der Minderheit sind ${ }^{3) 4}$ ).

Nach Abwägung aller Argumente dürten die Möglichkeiten regierungspolitischer Beeinflussung von Zentralbankentscheidungen über die personelle Besetzung ihrer zentralen Willenbildungsorgane als gering anzusehen sein. Zwar hat es in der Vergangenheit nicht an Versuchen gefehlt, die formelle Unabhängigkeit der Bundesbank einzu-

1) Vgl. hierzu auch Robert (1978), S. $21 \mathrm{ff}$.

2) Sie werden vom Bundespräsidenten auf Vorschlag des Bundesrates ernannt. Letzterer ist jedoch an die Vorschläge der jeweiligen Landesregierung gebunden. Vgl. Jarchow (1974), S. 60

3) Vgl. Huppertz (1977), S. 74 sowie Dickertmann (1975), S. 25 und Klasen (1970), S. 23

4) Kaiser sieht indessen in der Besetzungspraxis der Landeszentralbankpräsidien eine Beeinflussung der Notenbankautonomie, da die Landesregierungen dazu neigen, das Präsidentenamt mit verdienten Persönlichkeiten ihres Einflußbereichs zu besetzen, deren Sachkompetenz umstritten ist. Vgl. Kaiser (1980), S. $33 \mathrm{ff}$. Hahn hat in diesem Zusammenhang vorgeschlagen, daß jeweils die Hälfte der Sitze im Zentralbankrat Personen vorbehalten se in soll, "die eine erfolgreiche Bundesbank-Laufbahn nachweisen können". Hahn (1976), S. 528 
schränken ${ }^{1)}$, doch scheiterten derartige Versuche vor allem am Widerstand der offentlichkeit, in deren Augen eine autonome Bundesbank nach wie vor als Garant für eine wirksame Politik der Preisniveaustabilität angesehen wird ${ }^{2}$ ).

2.2.1.2. Koordinierungsverflechtung und Unabhängigkeit der Deutschen Bundesbank

Das Bundesbankgesetz verpflichtet die Zentralbank, die allgemeine Wirtschaftspolitik der Bundesregierung unter Wahrung ihrer Aufgabe zu unterstützen ${ }^{3)}$. Um dieser Verpflichtung nachkommen zu können, ist eine Koordinierung von Regierungs- und Zentralbankpolitik unumgänglich. Das Bundesbankgesetz selbst sieht daher im $\S 13$ eine institutionalisierte Zusammenarbeit zwischen Regierung und Bundesbank vor. Weitere institutionalisierte Kontakte bestehen durch die Teilnahme der Bundesbank an den Beratungen des Konjunkturrates ${ }^{4}$ ) und des Finanzplanungsrates ${ }^{5)}$. Hinzu kommt eine Vielzahl informeller Kontakte. So wurde die Bundesbank z.B. regelmäBig zu den Sitzungen der "Konzertierten Aktion" eingeladen und wirkt in verschiedenen interministeriellen Ausschüssen mit 6 ).

HANSMEYER sieht in diesem "Netz von institutionellen Verknüpfungen"7) eine Einengung des rechtlich bestehenden Handlungsspielrau-

1) Sie reichen von einer stärkeren Bindung der Zentralbank an die gesamtwirtschaftlichen Ziele der Bundesregierung über die Einrichtung einer Schiedsstelle im Konfliktfall (vgl. hierzu Ehrenberg (1976), S. 174) bis zur sogenannten Zwei-Schlüssel-Theorie, die für die Bundesbank neue Instrumente bei eingeschränkter Verfügungsgewalt vorsah. Vgl. Huppertz (1977), S. 78 f. Vgl. auch die bei Robert (1978), S. 57 ff. erläuterten Reformvorschläge.

2) Vgl. Ziffzer (1980), S. 502 sowie v. Spindler/Becker/Starke (1973), S. 265

3) Vgl. $\$ 12$ BBankG

4) $\mathrm{Vgl}$. 18 IV StabG

5) $\mathrm{Vgl} . \S 51 \mathrm{HGrG}$

6) Vgl. Kaiser (1980), S. 41, Fußnote 3

7) Hansmeyer (1968), S. 159 
mes der Notenbank" : "Der Handlungsspielraum einer Notenbank nimmt tendenziell ab, je mehr ein System koordinierender Maßnahmen für Informationen und Durchführung im Bereich der Konjunkturpolitik entwickelt wird ${ }^{2)}$."

Diese These trifft in einer Volkswirtschaft, in der interdependente wirtschaftspolitische Ziele von zwei rechtlich unabhängigen Instanzen verfolgt werden, selbstverständlich zu. Aus der Interdependenz des der Notenbank vorgegebenen Ziels der Preisniveaustabilität mit anderen wirtschaftspolitischen Zielen ergibt sich zwangsläufig eine Abhängigkeit der Zentralbank von der Regierung, die mit zunehmender Krisenanfälligkeit einer Volkswirtschaft immer stärker wird ${ }^{3)}$. Diese sich aus der Interdependenz wirtschaftspolitischer Ziele ergebende Abhängigkeit zwischen Zentralbank und Regierung ist jedoch zunächst als gegenseitige Abhängigkeit zu begreifen und sollte ohne Berucksichtigung weiterer Fakten nicht vorschnell als einseitige Abhängigkeit der Bundesbank interpretiert werden.

Man sollte die genannten Koordinierungsgremien daher zunächst als institutionalisierte Kontakte zweier rechtlich unabhängiger, aber aufgrund ihrer ziele interdependenter wirtschaftspolitischer Instanzen betrachten, die der Darlegung der jeweils eigenen Vorstellungen und der gegenseitigen Information dienen.

Die These, daB aus der Existenz von Koordinierungsgremien zwischen Bundesbank und Regierung nicht notwendigerweise die einseitge Abhängigkeit der Bundesbank folgt, wird z.B. durch die Vorgänge im Jahre 1972 belegt, als sich der damalige Bundesbankpräsident Klasen in einer Kabinettsitzung gegen Wirtschafts- und Finanzminister Schiller durchsetzte, obwohl er kein Stimmrecht besaß. Sollte die

1) Vgl. Hansmeyer (1968), S. $159 \mathrm{ff}$.

2) Ebenda, S. 160 (im Original gesperrt gedruckt). In diesem Sinne auch Caesar (1979), S. $147 \mathrm{f}$.

3) Vgl.auch Caesar (1979), S. 104, der in diesem Zusammenhang von einem zunehmenden Konfliktpotential der Geldpolitik spricht. 
Bundesbank in der Tat dem latenten Zwang ausgesetzt sein, zum "Finanzier der Finanzpolitik"1) zu werden - eine Frage, die im Rahmen der empirischen Analyse noch zu prüen sein wird -, so ist dies jedenfalls nicht auf die Existenz von Koordinierungsinstanzen zurückzuführen ${ }^{2)}$.

2.2.1.3. Die Unabhängigkeit des Federal Reserve System (FRS)

2.2.1.3.1. Die Struktur des amerikanischen Zentralbankensystems

Die Vereinigten Staten weisen ein zweistufiges Zentralbankensystem auf $^{3}$ ). Neben dem Board of Governors ${ }^{4}$ ) in Washington bestehen zwölf rechtlich selbständige regionale Federal Reserve Banks, die die Exekutivfunktion des Zentralbankensystems ausüben. Die für die Frage der Geldmengenwirkungen statilicher Budgetdefizite entscheidenden offenmarktoperationen werden von einer eigens hierfür geschaffenen Institution, dem "Federal Open Market Committee", durchgeführt, dem aufgrund seiner organisatorischen Stellung auch die Koordinierungsfunktion für den Einsatz der übrigen geldpolitischen Instrumente zukommt ${ }^{5}$ ). Die organisatorische struktur des amerikanischen Zentralbankensystems und die personellen Abhängigkeiten innerhalb dieser Struktur lassen sich anhand von Abbildung 7 veranschaulichen (siehe S. 112).

Oberstes Entscheidungsorgan und "real decision-making authority of the system ${ }^{6}$ )" ist der Board of Governors (BOG) in Washington. Er

1) Hansmeyer (1968), S. 161

2) In diesem Sinne auch Caesar (1979), S. 201: "Von einer Asymmetrie der Beratungskompetenzen zugunsten der Regierung kann demnach in der Realität keine Rede sein."

3) Vgl. zu den folgenden Ausführungen vor allem Braun (1969), S. $121 \mathrm{ff}$.

4) Der Board of Governors wird in Anlehnung an seine offizielle Bezeichnung bis zum ErlaB des Banking Act von 1935 oft auch als "Federal Reserve Board" beze ichnet. $\mathrm{Vgl}$. ebenda, S. 125, FuBnote 3

5) Vgl. ebenda, S. 136 f. sowie Whittlesey (1963), S. 37 und Luckett (1976), S. 267

6) Horvitz (1969), S. 272 


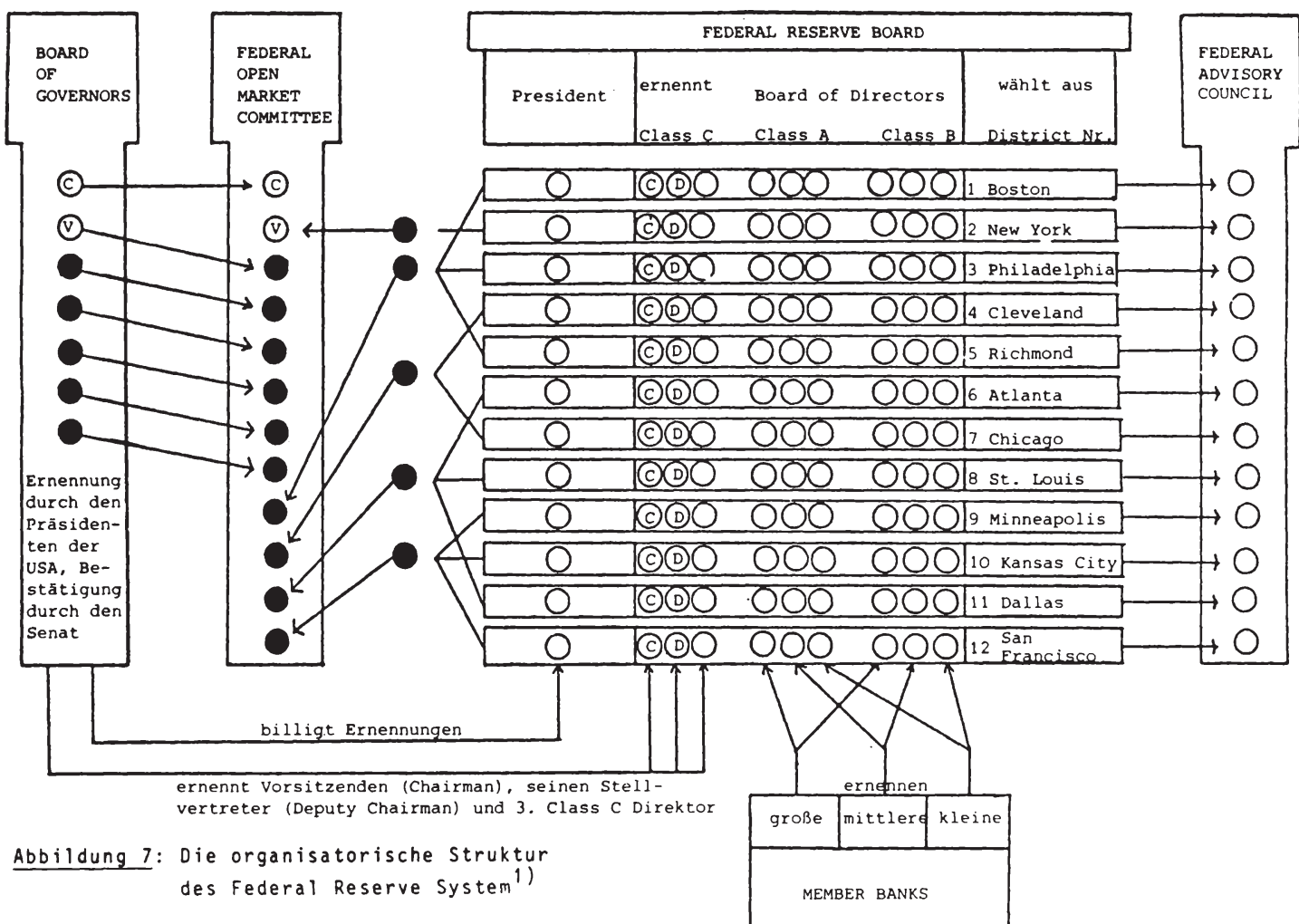

1) Quelle: Braun (1969), s. 126 
besteht aus sieben vom Präsidenten mit Zustimmung des Senats ernannten Mitgliedern, von denen eines - ebenfalls vom Präsidenten zum Vorsitzenden (chairman), ein zweites zu dessen Stellvertreter (vice chairman) bestimmt wird ${ }^{1}$ ). Die sieben Board-Mitglieder werden auf vierzehn Jahre gewählt. Dabei kommt eine Art rollierendes System zur Anwendung, gemäß dem alle zwei Jahre ein Mitglied neu gewählt wird. Chairman und Vice Chairman werden auf vier Jahre ernannt. Während eine Wiederwahl der Board-Mitglieder nach Ablauf der vierzehnjährigen Amtsperiode ausgeschlossen ist, darf ein Mitglied während dieser vierzehn Jahre mehrmals zum Chairman ernannt werden ${ }^{2}$ ).

Zweiter Träger der geldpolitischen Willensbildung ist das Federal Open Market Committee (FOMC), in dem die sieben Mitglieder des BOG und fünf der zwölf Präsidenten der Federal Reserve Banks stimmberechtigt sind. Den Vorsitz im FOMC führt der Vorsitzende des BOG, dem auch de facto die entscheidende stimme in diesem Gremium zukommt ${ }^{3)}$. Die den Regionalbanken. zustehenden Sitze werden jedes Jahr nach einem aus Abbildung 7 ersichtlichen Modus an die übrigen Federal Reserve Banks vergeben ${ }^{4}$ ).

Die Exekutivfunktion im amerikanischen Zentralbankensystem wird durch die Federal Reserve Banks (FRBs) ausgeubt, deren Board of Directors sich aus neun Mitgliedern zusammensetzt. Dabei sind drei jeweils drei Personen umfassende Klassen von Direktoren zu unterscheiden ${ }^{5}$. Die drei Class-C-Direktoren werden direkt vom BOG bestimmt, während die Class-A- und Class-B-Direktoren unter der Voraussetzung der Billigung durch den BOG von den privaten Mit-

1) Vgl. Horvitz (1969), S. 272 sowie Braun (1969), S. 129

2) Vgl. Horvitz (1969), S. 272 sowie Kaufmann (1973), S. 185

3) Vgl. Whittlesey (1963), S. 36 f. und Yohe (1968), S. 144

4) Vgl. Braun (1969), S. 135 und Luckett (1976), S. 272. Die übrigen Präsidenten der Federal Reserve Banks nehmen beratend an den Sitzungen des FOMC teil. Vgl. auch Borins (1972), S. 176

5) Vgl. Horvitz (1969), S. 273 
gliedsbanken der jeweiligen FRB gewählt werden ${ }^{1)}$. Den Vorsitz im Board of Directors führt einer der Class-C-Direktoren.

\subsection{Konsequenzen für die Zentralbankautonomie}

Die Darstellung der organisatorischen Struktur des amerikanischen Zentralbankensystems macht deutlich, daB dem BOG eine dominierende Stellung innerhalb des Notenbanksystems zukommt ${ }^{2}$ ). Diese Vorrangstellung ergibt sich vor allem aus der Tatsache, daB der BOG die Besetzung der übrigen Organe kontrollieren bzw. die dort getroffenen Entscheidungen durch seine Stimmenmehrheit direkt beeinflussen kann.

Eine Analyse der Zentralbankautonomie hat somit neben der Unabhängigkeit der Notenbank als Institution insbesondere die Frage der personellen Abhängigkeit der Mitglieder des BOG von der Regierung zu untersuchen.

\subsection{Personelle Unabhängigkeit}

Die vierzehnjährige Amtszeit und das Verbot der Wiederwahl deuten zunächst auf eine relativ autonome Position der einzelnen BoardMitglieder von der Regierung hin. Unabhängigkeitsverstärkend wirkt der Umstand, daB i.d.R. alle zwei Jahre nur ein Board-Mitglied neu bestellt wird, so daß der Präsident nicht einmal innerhalb zweier Amtsperioden eine Mehrheit im Board ernennen kann ${ }^{3}$ ).

1) Vgl. Z.B. Luckett (1976), S. 271. Die Mitgliedsbanken werden dabei vom BOG in drei größenabhängige Kategorien eingeteilt, die jeweils einen Vertreter der Class-A- und Class-B-Direktoren wählen.

2) Die dominierende Stellung des BOG wird in nahezu allen Lehrbüchern zur Geldund Bankpolitik betont. Vgl. z.B. Luckett (1976), S. 266, Culbertson (1972), S. 163 und Kent (1966), S. 403

3) $\mathrm{Vgl}$. Horvitz (1969), S. 272 
Da der Vorsitzende innerhalb des BOG erfahrungsgemäB die entscheidende Position einnimmt ${ }^{1)}$, spricht allerdings die Möglichkeit seiner Wiederwahl gegen die Unabhängigkeit des Gremiums. Vor allem in Zeiten, in denen die Wiederernennung des Chairman ansteht, kann eine gewisse Abhängigkeit von der Regierung a priori nicht von der Hand gewiesen werden ${ }^{2}$ ).

Die endgültige Beurteilung der im Prinzip möglichen Abhängigkeit des Board-Vorsitzenden von der Regierung hängt selbstverständlich entscheidend von der Person des Chairman ab ${ }^{3)}$. GenieBt dieser in der offentlichkeit großes Ansehen und den Ruf eines sachkundigen Experten, so wird er sich einer Einflußnahme seitens der Regierung erfolgreicher widersetzen können als ein "schwacher" Chairman").

Es bleibt jedoch festzuhalten, daß die personelle Unabhängigkeit des Board-Vorsitzenden nicht gesichert ist. Eine EinfluBnahme der amerikanischen Regierung auf Zentralbankentscheidungen ist daher nicht auszuschließen.

\subsection{Institutionelle Unabhängigkeit}

Neben die mögliche personelle Abhängigkeit des Board-Vorsitzenden tritt die Abhängigkeit der Zentralbank als Institution. Ausgangspunkt der Oberlegungen ist die Feststellung, daB das Federal Reserve Act im Falle des Konflikts zwischen Zentralbank und Regie-

1) Vgl. vor allem Whittlesey (1963), S. 34 f., der auf die starke Stellung der ehemaligen Vorsitzenden Eccles und Martin eingeht. Vgl. auch Simpson (1976), S. 193, der den Vorsitzenden als den "most equal of equals" bezeichnet sowie Caesar (1979), S. 334.

2) So weist We intraub ((1978), S. 352) darauf hin, daß der Vorsitzende Eccles 1948 nicht wiederernannt wurde, weil er dem Weißen Haus gegenüber nicht "kooperativ" genug war.

3) Vgl. Whittlesey (1963), S. 35 sowie Braun (1969), S. 152

4) So wurde der Vorsitzende Martin 1967 aufgrund seines Ansehens in der offentlichkeit wiederernannt, obwohl einfluBreiche Politiker die Wiederernennung verhindern wollten. Vgl. Braun (1969), S. $156 \mathrm{f}$. Dagegen wurden Arthur Burns 1978 aus eindeutig innenpolitischen Grüden nicht wiederernannt, obwohl dies von zahlreichen Vertretern der amerikanischen Wirtschaft gefordert worden war. Vgl. Caesar (1979), S. 332 
rung die Oberwachung und Kontrolle der dem FRS zustehenden Befugnisse durch das Schatzamt vorsieht ${ }^{1)}$. Diese Vorschrift hat jedoch in der Praxis keine Bedeutung. Sie wurde nicht einmal im Jahre 1951, als es zum offenen Konflikt zwischen Schatzamt und Präsident auf der einen und Zentralbank auf der anderen Seite kam und in dem die Regierung schließiich nachgeben mußte ${ }^{2)}$, angewandt. Die amerikanische Literatur geht daher zu Recht von der juristischen Unabhängigkeit des FRS aus ${ }^{3}$ ).

Weit stärker als diese noch aus der Urfassung des Federal Reserve Act stammende Vorschrift scheint jedoch der Umstand ins Gewicht zu fallen, daß der Kongreß die Unabhängigkeit des FRS durch Anderung des Federal Reserve Act beseitigen könnte ${ }^{4}$. Vor allem in Wahljahren scheint die institutionelle Unabhängigkeit des FRS gefährdet, wenn es eine mit der expansiven Fiskalpolitik der Regierung in Konflikt stehende kontraktive Geldpolitik betreibt. Für diese These spricht nicht zuletzt die Existenz einer politisch starken "lowinterest lobby" 5 ), welche den Kongreß und den Präsidenten in Wahljahren dazu veranlaBt, die offentlichkeit auf die durch "exzessive" Zinssätze bedingte Depressionsgefahr aufmerksam zu machen und eine expansive Geldpolitik zu fordern ${ }^{6}$ ). Wie stark der EinfluB dieser Interessengruppe ist, zeigt die im Jahre 1977 beschlossene Ergänzung des Federal Reserve Act, in dem der BOG und das FOMC auf die Ziele Vollbeschäftigung, Preisniveaustabilität und "moderate longterm interest rates" festgelegt wurden. Der ursprüngliche Senats-

1) Vgl. Sec. 10, $\S 6$ Federal Reserve Act

2) Vgl. hierzu Shapiro (1975), S. $316 \mathrm{ff}$. und Geisler (1953), S. $57 \mathrm{ff}$.

3) Vgl. auch Braun (1969), S. $147 \mathrm{f}$.

4) Vgl. Bach (1962), S. 272, Zecher (1971), S. 690 sowie Luckett (1976), S. 279, Kaufmann (1973), S. 191 und Simpson (1976), S. 194

5) "It is no accident that those sectors most vulnerable to increases in nominal interest rates have made them strong politically. The low-interest lobby is led by builders and construction unions and by financial institutions that earn their living by borrowing short to lend long." Kane (1980), S. 207

6) Vgl. Kane (1980), S. $207 \mathrm{f}$. 
entwurf hatte lediglich das Vollbeschäftigungs- und Preisniveaustabilitätsziel vorgesehen ${ }^{1}$ ).

Die tatsächliche Gefährdung der institutionellen Unabhängigkeit des FRS dürte zwar davon abhängen, welchen Wert die amerikanische offentlichkeit einer unabhängigen Zentralbank beimiBt. Die Tatsache, daB eine Vielzahl von okonomen und Politikern eine gesetzlich fixierte Unterordnung der Zentralbank unter die Regierung fordert ${ }^{2)}$, spricht jedoch ebenso wie das Fehlen eines der Zentralbank primär vorgegebenen Ziels der Preisniveaustabilität dafür, daß die amerikanische offentlichkeit weniger sensibel auf eine Einschränkung der Zentralbankautonomie reagieren würde als die deutsche ${ }^{3}$. Gerade die Deckungsgleichheit der Notenbankziele mit den wirtschaftspolitischen Zielen der Regierung dürte es der offentlichkeit angesichts des Fehlens einer eindeutigen Priorität für das Ziel der Preisniveaustabilität ${ }^{4}$ auch schwerer machen, die Notwendigkeit der Existenz einer zweiten, von der Regierung unabhängigen wirtschaftspolitischen Institution einzusehen. Lediglich in Perioden hoher Inflationsraten, wie z.B. im Konfliktjahr 1951, scheint die institutionelle Unabhängigkeit des FRS gesichert.

Ein Blick auf die Geldpolitik der USA seit 1951 erhärtet die These von der De-facto-Abhängigkeit der Notenbank. WEINTRAUB ${ }^{5}$ stellt in einer empirischen Analyse immer dann einen Wechsel in der Zielset-

1) $\mathrm{Vgl}$. den Abdruck des Resolutions- und des Gesetzestextes bei Weintraub (1978), S. $344 \mathrm{f}$. Als weiteres Beispiel für den EinfluB dieser Lobby kann der Umstand angefuihrt werden, daB der Kongre $B$ und das FRS in Hearings über die Frage diskutieren, wie der Bauindustrie in Zeiten hoher Zinsen am besten geholfen werden kann. Vgl. Zecher (1971), S. 681

2) Vgl. Z.B. Johnson (1964) und Bach (1962), vgl. auch Caesar (1979), S. 351 ff., der einen Uberblick über die in diesem Zusammenhang vorgebrachten Reformvorschläge gibt.

3) Die Unabhängigkeit der Notenbank scheint inzwischen "keineswegs mehr ein 'Tabu' und derart fest in der öffentlichen und akademischen Meinung verankert zu sein, daB" sie "auf Dauer eine starke politische Stütze für das FRS se in könnte". Caesar (1979), S. 363

4) Vgl. Sec. 2 A Federal Reserve Act

5) Vgl. Weintraub (1978), insbesondere S. $354 \mathrm{ff}$. 
zung und Zielrichtung der Geldpolitik fest, wenn ein neuer Präsident ins Amt $\left.\operatorname{kam}^{1}\right)$ : ". . We see that the Federal Reserve shifted course in the fundamental sense, easing or tightening significantly in 1953, 1961, 1969, 1971, 1974 und 1977. Except for 1971, these were years when the presidency also changed hands; and except for the changeover from President Kennedy to President Johnson, these were the only years when the presidency changed hands 2 ) " "From the Accord until now, much of the history of monetary policy can be explained just by noting who the president was when the policy under review was in effect ${ }^{3)}$."

Der EinfluB der Regierung, speziell des Präsidenten, auf das FRS wird schlieBlich durch mehrere Interviews deutlich, die mit Mitgliedern von Regierung und Zentralbank geführt wurden. Einige wichtige Passagen aus diesen Interviews finden sich im Anhang ${ }^{4}$.

Zusammenfassend läßt sich feststellen, daß die Unabhängigkeit der amerikanischen Zentralbank wohl mit Recht als "Unabhängigkeit innerhalb der Regierung" und nicht als Unabhängigkeit von der Regierung bezeichnet wird ${ }^{5}$. So genieBt das FRS aufgrund seiner Unabhängigkeit von Budgetbewilligungen durch den Kongre ${ }^{6}{ }^{6}$ sicherlich einen gewissen Freiheitsspielraum und aufgrund der Sachkompetenz seiner Mitglieder sicherlich auch einen partiellen EinfluB auf einzelne Regierungsentscheidungen. Im Falle eines Konflikts mit der generellen Zielrichtung der Wirtschaftspolitik der Regierung in Wahljahren dürte die Unabhängigkeit des FRS jedoch nicht allzu groB sein. Diese Aussage gilt trotz der Tatsache, daB die Notenbank in einigen Konfliktfällen durch von den Regierungsforderungen

1) Vgl. auch Potts/Luckett (1978), S. 132 und Friedlaender (1973), S. 42 f., deren Ergebnisse die Hypothese von Weintraub bestätigen.

2) Weintraub (1978), S. 349

3) Ebenda, S. 356

4) Vgl. Anhang, Punkt 1.

5) Vgl. Braun (1969), S. 148 und Kaufmann (1973), S. 191

6) Vgl. Luckett (1976), S. 279 
abweichende Maßnahmen zeitweilig ihre Unabhängigkeit demonstrier$t^{1)}$. Am treffendsten wird die Situation des FRS durch BORINS charakterisiert, der feststellt, daß die Mitglieder der amerikanischen Zentralbank gerade deshalb von den Politikern abhängig sind, weil sie ihre de jure unabhängige Stellung bewahren wollen: "The Federal Reserve System nominally is the most independent central bank in the western world, as its officials are well aware. They wish to maintain this independence. Paradoxically, to maintain this general independence to set monetary policy, they may well give up their particular independence at any one time by choosing to follow policies which the Congress and the President would $1 \mathrm{ike} \mathrm{e}^{2)}$."

\subsubsection{SchluBfolgerungen aus der institutionellen Analyse der} Zentralbankabhängigkeit

Die im Rahmen der institutionellen Analyse der Zentralbankautonomie in der Bundesrepublik Deutschland und in den USA diskutierten Argumente führen zu dem SchluB, daB die Unabhängigkeit der Deutschen Bundesbank stärker gesichert ist als die Unabhängigkeit des Federal Reserve System. Der Grund für die stärkere Unabhängigkeit der Deutschen Bundesbank düfte vor allem in der Bedeutung liegen, die einer unabhängigen Zentralbank als Garant eines stabilen Geldwerts in den Augen der deutschen offentlichkeit zukommt und in der Tatsache, daB die Deutsche Bundesbank seit ihrem Bestehen mit einem eindeutigen Mandat zur Sicherung der Währung ausgestattet ist.

1) Vgl. hierzu Caesar (1979), S. $336 \mathrm{ff}$, der jedoch feststellt, daß die Notenbank " - bei aller temporären Konfliktbereitschaft - die große Linie ihrer Politik durchaus auf die Zielprioritäten der Regierung abgestimmt zu haben" scheint (S. 343). "DaB an der juristischen Stellung des FRS . . . bislang nichts geändert worden ist, ist wesentlich dem vergleichsweise geringen Konfliktpotential der Geldpolitik zuzuschreiben gewesen (S. 360)."

2) Borins (1972), S. 185 f. Vgl. auch Erb (1978a), S. 5. Kane (1980), S. 209 geht sogar so weit zu behaupten, da $\beta$ "the Fed is an institution designed by politicians to serve politicians". 


\subsubsection{Empirische Analyse}

Bisher vorliegende empirische Analysen untersuchen jeweils Teilaspekte der von BW postulierten Zusammenhänge. Im Rahmen dieser Arbeit sollen zunächst zwei grundlegende Ansätze einer positiven Analyse des Zentralbankverhaltens in der Bundesrepublik Deutschland, die beide Zweifel an der Unabhängigkeit der Deutschen Bundesbank aufkommen lassen, in ihren Grundzügen dargestellt und einer vergleichenden Kritik unterzogen werden. Die Kritik beider Ansätze macht deutlich, daß die in beiden Modellen gezogene Schlußfolgerung einer De-facto-Abhängigkeit der Deutschen Bundesbank nicht zwingend ist. Vielmehr kann mit Hilfe eines alternativen Satisfizierungsansatzes am Beispiel der Diskontpolitik der Deutschen Bundesbank von 1969 - 1980 gezeigt werden, daB sich auch die gegenteilige Auffassung einer de facto autonomen Bundesbank empirisch belegen läßt. Die Streitfrage läßt sich dann zum großen Teil auf die Diskussion über die Bedingungen reduzieren, unter denen ein Konflikt zwischen Regierung und Bundesbank vorliegt ${ }^{1}$ ).

Abschnitt V.2.2.2.2. ist der empirischen Analyse der Geldmengenund Inflationswirkungen staatlicher Budgetdefizite gewidmet. Der Diskussion der für den Bereich der Vereinigten Staaten durchgeführten Untersuchungen folgt der empirische Test der Geldmengenwirkungen von Budgetdefiziten in der Bundesrepublik Deutschland.

1) Auf ein drittes Model1, das von Cowart (1978) vorgelegt wurde, wird hier nicht eingegangen. Cowart untersucht zwar für 7 europäische Staaten den EinfluB ökonomischer und politischer Variablen auf die Diskontpolitik, ist aber primär an der Frage interessiert, ob rechte oder linke Regierungen einen stärkeren EinfluB auf die Diskontpolitik ausüben. Für die Analyse der Bundesbankautonomie scheint dieser Ansatz daher nicht geeignet, da die Abhängigkeit der Notenbank nicht untersucht wird, sondern als A-priori-Annahme in das Modell eingeht. Oberdies deutet die Tatsache, daß nur wenige Koeffizienten statistisch signifikant von Null verschieden sind, auf eine nicht exakte Modellspezifikation hin. 
2.2.2.1. Empirische Analysen der Zentralbankautonomie in der Bundesrepublik Deutschland

Die im folgenden zu diskutierenden Ansätze unterscheiden sich vor allem hinsichtlich der Annahmen über das grundsätzliche Verhalten der Deutschen Bundesbank. Während BASLER ${ }^{1)}$ in seinem Modell von einem Optimierungsverhazten der Zentralbank unter Nebenbedingungen ausgeht, unterstellen FREY und SCHNEIDER ${ }^{2)}$ eine konfliktvermeidende Satisfizierungsstrategie der Bundesbank.

2.2.2.1.1. BASLERs Optimierungsmode 11 zur Bestimmung von Zielprioritäten der Deutschen Bundesbank

Die Arbeit BASLERs, deren prinzipieller Ansatz im folgenden dargestellt werden soll, stellt die Weiterentwicklung einer Reihe von Beiträgen ${ }^{3)}$ dar, die auf der Grundlage des Ansatzes von THEIL ${ }^{4}$ ) das Verhalten wirtschaftspolitischer Instanzen untersuchen, denen die Optimierung ihrer Nutzenvorstellungen unter bestimmten, durch die Wirtschaftsstruktur gegebenen Nebenbedingungen unterstellt wird. Die Nutzenvorstellungen der wirtschaftspolitischen Instanzen werden in diesen Ansätzen meist durch eine Nutzenverlustfunktion wiedergegeben, die wirtschaftspolitische Ziele und Instrumente als Variable enthält und deren Minimierung von der jeweiligen Instanz angestrebt wird ${ }^{5}$ ).

Dabei wird angenommen, die Wirtschaftspolitiker hätten eine Vorstellung von für sie idealen Ziel- ( $\left.z^{*}\right)$ und Instrumentenwerten ( $i^{*}$ ) und betrachteten Abweichungen der tatsächlichen Ziel- ( $z$ ) und Instrumentenwerte $(i)$ von diesen. Wunschwerten als Nutzenverlust. Auf

1) Vgl. Basler (1979) und (1978)

2) Vgl. Frey/Schneider (1978b) und Schneider (1979)

3) Vgl. vor allem Friedlaender (1973), aber auch Wood (1967) und Pissarides (1972)

4) Vgl. The il (1964), S. $1 \mathrm{ff}$. und S. $32 \mathrm{ff}$.

5) Vgl. aber Friedlaender (1973), S. 26 ff., die die Maximierung einer Nutzenfunktion unterstellt. 
der Basis dieses 0ptimierungskalküls lassen sich Ziel- und Instrumentpräferenzen der Wirtschaftspolitiker empirisch schätzen.

BASLER verbessert die bisherigen Optimierungsansätze nicht nur methodisch, sondern geht noch einen Schritt weiter: Er ermittelt die Ziel- und Instrumentpräferenzen der Deutschen Bundesbank, vergleicht ihre Zielprioritäten mit denen der Regierung und zieht aus diesem Vergleich Schlußfolgerungen bezüglich der Bundesbankautonomie.

Im einzelnen geht BASLER wie folgt vor: Er unterstellt wie die Mehrzahl der übrigen Optimierungsmodelle einen mit zunehmender $A b-$ weichung $\left(z-z^{*}\right)$ bzw. ( $\left.i-i^{*}\right)$ überproportional zunehmenden Nutzenverlust und bringt dies durch eine quadratische Nutzenverlustfunktion ${ }^{1)}$ zum Ausdruck, die im Falle von m Zielen und $n$ Instrumenten die folgende Form hat:

(V. 1) $L=\sum_{j=1}^{m} w_{j}\left(z_{j}-z_{j}{ }^{*}\right)^{2}+\sum_{l=1}^{n} v_{1}\left(i_{1}-i_{1}{ }^{*}\right)^{2}$.

$w_{j}$ und $v_{1}$ sind die Gewichte der quadratischen Ziel- und Instrumentabweichungen und dienen als "Maß für die Ziel-bzw. Instrumentpräferenzen der Wirtschaftspolitiker"2). Die Nebenbedingungen sind durch die Wirtschaftsstruktur, in der Arbeit von BASLER genauer gesagt durch die Vorstellungen der Entscheidungsträger der Deutschen Bundesbank über die Wirkung ihrer Instrumente auf die angestrebten Zielgrößen ${ }^{3)}$, gegeben und lassen sich wie folgt darstellen:

(v.2) $\quad z_{j}=\sum_{l=1}^{n} \gamma_{j l} \cdot i_{1}+u_{j} \quad j=1, \ldots, m$.

1) Vgl. hierzu Theil (1964), S. 2 ff.

2) Basler (1979), S. 10

3) Vgl. ebenda, S. $10 \mathrm{f}$. 
Die $\gamma_{j l}$ werden als Strukturkoeffizienten bezeichnet und stellen ein MaB für die Vorstellung der Wirtschaftspolitiker über die Wirkung des Instruments 1 auf die ZielgröBe $j$ dar, die $u_{j}$ sollen die nicht explizit aufgeführten exogenen Einflüsse auf die Zielgrößen repräsentieren ${ }^{1)}$.

Aus der Optimalbedingung

(v.3) $\quad \frac{\partial L}{\partial i_{1}}=2 \sum_{j=1}^{m} w_{j} \gamma_{j 1}\left(z_{j}-z_{j}^{*}\right)+2 v_{1}\left(i_{1}-i_{1}{ }^{*}\right)=0$

ergibt sich

(v.3.1) $\quad i_{1}=i_{1} \star-\sum_{j=1}^{m} \alpha_{1 j}\left(z_{j}-z_{j}^{*}\right)$.

Gleichung (V.3.1) wird von BASLER als Reaktionsfunktion der Zentralbank auf Zielabweichungen, der Ausdruck

(v.4) $\quad a_{1 j}=\frac{w_{j} \cdot \gamma_{j 1}}{v_{1}}$

als Reaktionskoeffizient bezeichnet ${ }^{2)}$.

Diese Reaktionskoeffizienten können ökonometrisch geschätzt werden. Aufgrund der multiplikativen Verknüpfung der Ziel- und Instrumentgewichte mit den Strukturkoeffizienten ist es möglich, für gegebene Strukturkoeffizienten relative ziel- und Instrumentgewichte als MaB für die Präferenzen der Bundesbank zu ermitteln.

Dazu bedarf es zunächst der Auffindung der Strukturkoeffizienten $\gamma_{j l}$. BASLER legt die Strukturkoeffizienten auf der Basis einer Textexegese von Bundesbankkommentaren ${ }^{3)}$ fest. Selbstverständich

1) Vgl. Basler (1979), S. 13

2) Vgl. ebenda, S. 23

3) Vgl. ebenda, S. $62 \mathrm{ff}$. 
lassen sich die Strukturkoeffizienten nicht exakt quantifizieren. Geht aus den Bundesbankkommentaren jedoch hervor, daB die Bundesbank Instrument $i_{1}$ zur Beeinflussung des Ziels $z_{1}$ für wirksamer hält als Instrument $i_{2}$, so sieht BASLER die Strukturvorstellungen durch die Quotienten $\gamma_{11} / \gamma_{11}=1$ und $\gamma_{12} / \gamma_{11}=0,5$ als annäherungsweise quantifiziert an ${ }^{1}$.

Die Ermittlung der relativen Ziel- und Instrumentgewichte läBt sich nun recht einfach für den beispielhaft gewählten Fall von zwei Instrumenten zeigen ${ }^{2}$. Dazu werden die Reaktionskoeffizienten zunächst in eine Matrix eingetragen:

\begin{tabular}{c|c|c} 
& $z_{1}$ & $z_{2}$ \\
\hline$i_{1}$ & $\frac{w_{1}}{v_{1}} \cdot r_{11}$ & $\frac{w_{2}}{v_{1}} \cdot r_{21}$ \\
$i_{2}$ & $\frac{w_{1}}{v_{2}} \cdot r_{12}$ & $\frac{w_{2}}{v_{2}} \cdot r_{22}$
\end{tabular}

Durch Division jedes Elements $\alpha_{1 j}$ durch die aus der Textexegese ermittelten Strukturkoeffizientenverhältnisse $\frac{\gamma_{j l}}{\gamma_{11}}$ erhält man die
Matrix

\begin{tabular}{c|c|c|c} 
& $z_{1}$ & $z_{2}$ & $\sum$ \\
\hline$i_{1}$ & $\frac{w_{1}}{v_{1}} \cdot r_{11}$ & $\frac{w_{2}}{v_{1}} \cdot r_{11}$ & $\frac{r_{11}}{v_{1}} \sum_{j=1}^{2} w_{j}$ \\
$i_{2}$ & $\frac{w_{1}}{v_{2}} \cdot r_{11}$ & $\frac{w_{2}}{v_{2}} \cdot r_{11}$ & $\frac{r_{11}}{v_{2}} \sum_{j=1}^{2} w_{j}$ \\
\hline$\Sigma$ & $w_{1} \gamma_{11} \cdot \sum_{l=1}^{2} 1 / v_{1}$ & $w_{2} \gamma_{11} \cdot \sum_{l=1}^{2} 1 / v_{1}$ &
\end{tabular}

1) Vgl. Basler (1979), S. 47

2) Vgl. auch ebenda, S. $48 \mathrm{ff}$. 
Aus den Spaltensummen lassen sich jetzt die relativen Zielgewichte, aus den Zeilensummen die relativen Instrumentgewichte ermitteln. Division der zweiten Spaltensumme durch die erste ergibt be ispielswe ise

$$
\frac{w_{2} \gamma_{11} \cdot \sum_{1=1}^{2} 1 / v_{1}}{w_{1} \gamma_{11} \cdot \sum_{1=1}^{2} 1 / v_{1}}=\frac{w_{2}}{w_{1}},
$$

Division der zweiten Zeilensumme durch die erste ergibt

$$
\text { (v.6) } \frac{\frac{r_{11}}{v_{2}} \cdot \sum_{j=1}^{2} w_{j}}{\frac{r_{11}}{v_{1}} \cdot \sum_{j=1}^{2} w_{j}}=\frac{v_{1}}{v_{2}} .
$$

Kehren wir zum allgemeinen Fall von $m$ Zielen und in Instrumenten zuruick, so bietet es sich an, jeweils ein ziel und ein Instrument als Standardziel bzw. - instrument mit dem Gewicht 1 anzunehmen. Für gegebene Strukturkoeffizientenverhältnisse läßt sich dann eine Prioritätenskala für die Ziele und Instrumente der Zentralbank aufstellen ${ }^{1)}$.

Wie bereits erwähnt, soll an dieser Stelle nur das Grundprinzip des BASLER-Ansatzes dargestellt werden. Durch Einbau einer nichtgeometrischen Lag-Struktur ${ }^{2}$, Annahme in der Zeit variabler Strukturvorstellungen der Wirtschaftspolitiker ${ }^{3)}$, Wahl einer asymmetri-

1) Anhand der ermittelten Zielgewichte lassen sich auch Zielabweichungen berechnen, die für die Zentralbank denselben Nutzenverlust bedeuten ( $\mathrm{vgl}$. Basler (1979), S. 52). Kennzeichnet $L_{1}$ den Nutzenverlust, der aus der Abweichung des Standardziels $z_{1}$ vom Wunschwert $z_{1}$ * um eine Zieleinheit resultiert, so beträgt die Indifferenzabweichung für das Ziel $\mathrm{j}$ : $\left|z_{j}-z_{j}{ }^{*}\right|=\left(L_{1} / w_{j}\right)^{1 / 2}$.

2) Vgl. ebenda, S. $33 \mathrm{ff}$.

3) Vgl. ebenda, S. $37 \mathrm{f}$. 
schen Verlustfunktion ${ }^{1)}$ und Normierung der Ziel- und Instrumentvariablen ${ }^{2)}$ verbessert BASLER die bisher zu diesem Problemkreis erschienenen und im Grundprinzip mit dem oben dargestellten Modell übereinstimmenden Ansätze entscheidend.

So berücksichtigt eine asymmetrische Verlustfunktion den Sachverhalt, daB die Obererfuillung eines Ziels um eine Zieleinheit in der Regel zu geringeren Nutzenverlusten führt als eine Untererfüllung des Ziels um eine Einheit ${ }^{3)}$. Eine Normierung der Ziel- und Instrumentvariablen ist dagegen erforderlich, weil letztere unterschiedliche Schwankungsbreiten und unterschiedliche Dimensionen haben können. Eine durch eine einprozentige Erhöhung der Preissteigerungsrate ausgelöste Mindestreservesatzvariation ist beispielswe ise nicht unmittelbar mit einer durch einen DevisenzufluB von 1 Mrd. DM bedingten Diskontsatzänderung vergleichbar ${ }^{4)}$. Um aussagefähige und vergleichbare Reaktionskoeffizienten zu erhalten, normiert BASLER daher die Ziel-und Instrumentvariablen mit den entsprechenden Standardabweichungen. Die in der Zielfunktion enthaltenen normierten Variablen $z_{j}^{n}$ und $i_{1}^{n}$ haben dann formal folgendes Aussehen ${ }^{5}$ ):

$$
\begin{aligned}
& \text { (v.7) } z_{j}^{n}=\frac{z_{j}}{\sigma_{z_{j}}} \\
& \text { (v.8) } \quad i_{1}^{n}=\frac{i_{1}}{\sigma_{i_{1}}} .
\end{aligned}
$$

1) Vgl. Basler (1979), S. $36 \mathrm{f}$.

2) Vgl. ebenda, S. $41 \mathrm{f}$.

3) Vgl. auch Friedlaender (1973), S. 28, die diesen Sachverhalt durch eine aus quadratischen und linearen Termen bestehende Nutzenfunktion berücksichtigt.

4) Vgl. Basler (1979), S. 32

5) Vgl. ebenda, S. 42 
Die genannten Erweiterungen stellen wichtige methodische Verbesserungen gegenüber den bisherigen, vom selben Grundansatz ausgehenden Modellen dar.

Auf der Basis des hier in den Grundzügen dargestellten Ansatzes ermittelt BASLER für den Zeitraum von 1958 bis 1974 die Ziel-und Instrumentpräferenzen der Deutschen Bundesbank. Die - auch für einige Unterzeiträume ermittelten - Zielpräferenzen sind in Tabelle 9 zusammengefaBt ${ }^{1)} 2$ ).

\begin{tabular}{|l|llll|}
\hline \multirow{2}{*}{ Analysezeitraum } & \multicolumn{4}{|c|}{ Relative } \\
\cline { 2 - 5 } & W WS & W $_{\text {ZG }}$ & WWW $_{\text {WW }}$ & W $_{\text {VB }}$ \\
\hline $1958 / 1-1974 / 12$ & 1,00 & 0,25 & 0,72 & - \\
$1958 / 1-1967 / 6$ & 1,00 & 3,82 & 0,32 & - \\
$1967 / 7-1974 / 12$ & 1,00 & 0,09 & 0,21 & - \\
$1969 / 10-1974 / 12$ & 1,00 & 0,06 & 0,97 & - \\
\hline
\end{tabular}

Tabelle 9: Zielpräferenzen der Deutschen Bundesbank 1958 bis 1974

Es wird deutlich, daB die Deutsche Bundesbank während des gesamten Zeitraumes dem Ziel der Preisniveaustabilität Vorrang vor allen anderen Zielen einräumte. Lediglich im Unterzeitraum von 1958 bis Mitte 1967 war eine Vorrangstellung des Zahlungsbilanzziels zu verzeichnen, was auf den Zielkonflikt zwischen innerer und äuBerer Stabilität zu Zeiten fester Wechselkurse zurückzuführen sein düf$t^{3}{ }^{3}$. Noch deutlicher wird dieser Zielkonflikt, wenn die Reaktionen der Bundesbank lediglich im Falle der Zieluntererfillung beobachtet werden. In diesem Fall rückt das Zahlungsbilanzziel im Gesamtzeitraum und in der ersten Teilperiode an die erste Stelle. BASLER

1) Vgl. Basler (1979), S. 84. Die Abkürzungen bedeuten: PS = Preisniveaustabilität, $Z G=$ Zahlungsbilanzgleichgewicht, WW = Wirtschaftswachstum, $V B=$ Vollbeschäftigung.

2) Hinsichtlich des Vollbeschäftigungsziels konnte Basler keine interpretierbaren Bundesbankreaktionen beobachten. Daher war keine Aussage über das relative Gewicht des Beschäftigungsziels möglich. Vgl. ebenda, S. 85

3) Vgl. ebenda 
sieht durch diese Zielgewichtung das Bemühen der Bundesbank bestätigt, die Voraussetzung zur Eindämmung inflatorischer Tendenzen durch auBenwirtschaftliche Absicherung zu schaffen ${ }^{1}$ ).

In der Tat düfte die Politik der Bundesbank durch diese Zielsetzung korrekt umschrieben sein, da in Zeiten fester Wechselkurse und hoher Inflationsraten im Ausland jede nur binnenwirtschaftlich orientierte Politik der Preisniveaustabilität erneut Exportimpulse auslösen und damit nicht nur das Zahlungsbilanzziel stören, sondern auch jegliche neuerliche Politik der Preisniveaustabilität im Innern erschweren würde. Anders ausgedrückt: Je besser. es gelungen wäre, Preisniveaustabilität im Inland zu erreichen, desto stärkere Inflationspotentiale wären von auBenwirtschaftlicher Seite entstanden, desto schwieriger wäre eine künftige Politik der Preisniveaustabilität geworden. Aufgrund dieser Ergebnisse kann der Bundesbank daher nicht der Vorwurf gemacht werden, sie habe den ihr gesetzlich vorgeschriebenen Auftrag nicht erfüllt.

Dennoch wird dieser Vorwurf von BASLER in anderem Zusammenhang erhoben. Wie bereits erwähnt, vergleicht BASLER die von ihm ermittelten Zielpräferenzen mit den ideologischen Zielpräferenzen politischer Parteien ${ }^{2)}$. Er kommt dabei zu dem Schiuß, daß die Bundesbankpräferenzen nicht unabhängig von den Parteienpräferenzen waren und daß diese Interdependenz vor allem zu Beginn der Siebziger Jahre zu einer starken Förderung des nur wenig gefährdeten, aber von der SPD/FDP-Regierung favorisierten Wachstumsziels seitens der Bundesbank geführt hat. Die Tatsache, daß zur selben Zeit das Ziel der Preisniveaustabilität relativ stärker gefährdet war, veranlaBt BASLER zu der These, die Bundesbank habe sich in dieser Zeit "nicht als die unabhängige Hüterin der Währung" ${ }^{3)}$ erwiesen.

1) Vgl. Basler (1979), S. 88

2) Vgl. hierzu Kirschen/Benard/Besters (1964), S. $224 \mathrm{ff}$.

3) Basler (1979), S. 90. Vgl. auch Basler (1978), S. 101 
Diese These ist jedoch in Anbetracht sowonl der Ergebnisse als auch des Ansatzes von BASLER zu relativieren. Zunächst einmal ist zu bemerken, daB das von ihm herangezogene ideologische Zielprioritätenschema für sozialdemokratische Parteien das Vollbeschäftigungsziel vor dem Wirtschaftswachstum und der Preisniveaustabilität als wichtigstes Ziel ausweist ${ }^{1)}$. Die Tatsache, daB BASLER durch seine ökonometrischen Untersuchungen eine Gewichtung des Beschäftigungsziels seitens der Bundesbank gar nicht ermitteln kann, muB daher angesichts der wirtschaftspolitischen Bedeutung des Vollbeschäftigungsziels jeden Vergleich mit einem das Beschäftigungsziel beinhaltenden Prioritätenschema der Regierung relativieren.

Im Hinblick auf den Ansatz BASLERs muB weiterhin bemängelt werden, daB die auf der Basis eines Optimierungskalkijls und empirischer Untersuchungen ermittelten Zielpräferenzen der Zentralbank mit "ideologischen" Zielpräferenzen der Parteien verglichen werden. Ein Vergleich ideologischer Zielpräferenzen der Regierung mit den empirisch ermittelten Zentralbankpräferenzen übersieht jedoch den Sachverhalt, daB die von der Regierung tatsächlich verfolgte Wirtschaftspolitik nicht nur von ihrer Ideologie, sondern auch von der Einschätzung alternativer wirtschaftspolitischer Ziele durch die Wähler abhängt ${ }^{2}$. Eine Analyse des Konflikts zwischen Zentralbankpräferenzen und ideologischen Regierungspräferenzen ist daher nur in Situationen sinnvoll, in denen die Regierung die Möglichkeit hat, ihre von den Wählerpräferenzen möglicherweise abweichenden ideologischen ziele tatsächlich zu verfolgen.

Eine aussagefähige Analyse der Unabhängigkeit der Bundesibank müBte daher die Regierungspräferenzen ebenfalls auf der Grundlage eines ökonomischen Kalküls, gegebenenfalls unter Berücksichtigung interdependenter Zielfunktionen von Regierung und Zentralbank, ermitteln.

1) Vgl. Basler (1979), S. 89

2) Vgl. Z.B. Frey (1977), S. $185 \mathrm{ff}$. 
SchlieBlich erhebt sich die Frage, ob es der Zentralbank angesichts der bestehenden Unsicherheit über die tatsächliche Wirtschaftsstruktur und die bestehenden Interdependenzen überhaupt möglich ist, die optimierung ihrer Nutzenvorstellung zu erreichen, oder ob sie sich nicht mit einer Satisfizierungsstrategie zufriedengeben muB ${ }^{1)}$.

Der Ansatz von BASLER kann somit zu einer umfassenden Beurteilung der Zentralbankautonomie nur teilweise herangezogen werden ${ }^{2)}$. Aus diesem wohl am weitesten entwickelten optimierungsansatz können zwar die Zielpräferenzen der Bundesbank bezüglich der drei Ziele Preisniveaustabilität, Zahlungsbilanzgleichgewicht und Wirtschaftswachstum abgeleitet werden. Die Nichtberücksichtigung des Beschäftigungsziels steht jedoch der Eignung des BASLER-Modells zur Einschätzung der Bundesbankautonomie genauso entgegen wie die Tatsache, daB weder die von BASLER unterstellte Nutzenverlustfunktion, noch die Nebenbedingungen ökonomisch begründbare Zielpräferenzen der Regierung beinhalten.

\subsection{Der Satisfizierungsansatz von FREY und SCHNEIDER}

Zwei der gegen das Modell von BASLER vorgetragenen Kritikpunkte werden in den Modelien von SCHNEIDER ${ }^{3)}$ und FREY/SCHNEIDER ${ }^{4}$ ) berücksichtigt. Zum einen wird auf die Interdependenzen zwischen Regierung, Zentralbank und Wählern abgestellt, wobei die Abhängigkeit der Regierung von den Wählerpräferenzen durch eine Popularitätsfunktion zum Ausdruck kommt, zum anderen wird die Annahme der Nutzenverlustminimierung unter Nebenbedingungen durch die Annahme ei-

1) Vgl. Schneider (1979), S. 476 f. sowie Frey/Schneider (1978b), S. 5. Vgl. aber auch die grundsätzlichen Bemerkungen von Basler zu einer optimalen Wirtschaftspolitik bei Unsicherheit. Vgl. Basler (1979), S. $15 \mathrm{ff}$. Sowie Brainard (1967)

2) An dieser Stelle ist anzumerken, daB Basler mit seiner Arbeit wohl nicht primär auf eine Beurteilung der Bundesbankautonomie abzielte. Dennoch erhebt Basler in seinem Fazit (S. 90) den Vorwurf der Abhängigkeit.

3) Vgl. Schneider (1979)

4) Vgl. Frey/Schneider (1978b) 
ner konfliktvermeidenden Satisfizierungsstrategie seitens der Zentralbank ${ }^{1)}$ ersetzt.

Gemäß dieser Strategie sind zwei Situationen zu unterscheiden, denen sich die Zentralbank gegenübersehen kann: die Konfliktsituation und die Situation des Nicht-Konflikts mit der Regierung. Erstere ist gegeben, wenn die Zentralbank eine kontraktive (expansive), die Regierung aber eine expansive (kontraktive) Politik betreibt ${ }^{2)}$.

Dabei wird die Richtung der Zentralbankpolitik durch die Veränderung der freien Liquiditätsreserven $L_{t}-L_{t-1}$, die Richtung der $R e-$ gierungspolitik durch die Differenz des aktuellen ( $B^{a}$ ) und des konjunkturneutralen Budgetsaldos $\left(B^{n}\right)$ gemessen $\left.{ }^{3}\right)$. FREY und SCHNEIDER postulieren nun, daB sich die Zentralbank im Konfliktfall immer an der Regierungspolitik ausrichten, im Falle des Nicht-Konflikts immer eine kontraktive Politik betreiben wird. Der Test dieser Hypothese erfolgt anhand eines ökonometrischen Modells für die Bundesrepublik Deutschland, dem Modellgleichungen der folgenden Art zugrundeliegen ${ }^{4}$ ):

(V.9) IZB ${ }_{t}^{i}=a_{0}+a_{1} \cdot I Z B_{t-1}^{i}+a_{2} \cdot(1-C) \cdot I D E O L Z B B_{t-2}+a_{3} \cdot C \cdot P O L R E G_{t-2}+u_{t} \cdot$

Gemäß der Gleichung ( $V .9$ ) wird postuliert, der Einsatz des i-ten Zentralbankinstruments $I Z B^{i}$ werde von einer Konstanten $a_{0}$, dem Ein-

1) Vgl. Schneider (1979), S. 476 f. und Frey/Schneider (1978b), S. 5. Vgl. auch den für diesen Ansatz grundlegenden Beitrag von Simon (1955) sowie Mosley (1976).

2) Die Autoren sehen den Konfliktfall auch gegeben, wenn die Bundesbank eine expansive, die Regierung eine kontraktive Politik betreibt. Vgl. Frey/Schneider (1978b), S. 6. Diese Möglichkeit scheint jedoch aufgrund ihrer Annahmen ausgeschlossen, da die Zentralbank annahmegemäB immer bemüht ist, eine kontraktive Politik zu verfolgen. Vgl. Schneider (1979), S. 478 und Frey/Schneider $(1978 b)$, S. 5

3) Eine expansive Zentralbankpolitik wird bei $L_{t}-L_{t-1}>0$, eine expansive Regierungspolitik bei $B^{a}-B^{n}>0$ betrieben. Vgl. Frey/Schneider (1978b), S. 6 und Schneider (1979), S. 477

4) Die Indizes $t-\tau$ sind Zeitindizes und bringen die von den Autoren postulierte Lag-Struktur zum Ausdruck. 
satz des Instruments in der Vorperiode ${ }^{1)}$, einer zufälligen Störvariablen $u_{t}$, der Politik der Regierung (POLREG) und der Ideologie der Zentralbank (IDEOLZB) beeinfluBt. Dabei wird weiter angenommen, die Ideologie der Bundesbank sei auf eine Beeinflussung der Inflationsrate $\hat{\mathbf{p}}$ gerichtet ${ }^{2)}$, d.h. es gilt:

(v.10) IDEOLZB $=\hat{\mathrm{p}}$.

Zur Formuleriung der Annahme, die Regierungspolitik beeinflusse den Instrumenteneinsatz nur im Konfliktfall, während im Nicht-Konfliktfall die Ideologie der Zentralbank zum Tragen komme, enthält die Modellgleichung die Dummy-Variable C, die im Konfliktfall den Wert 1, im Fall des Nicht-Konflikts den Wert 0 annimmt.

Die Modelle enthalten des weiteren eine Gleichung für den Einsatz finanzpolitischer Instrumente der Regierung IREG, die in der folgenden Art angenommen wird:

$$
I_{R E G_{t}^{j}}^{j}=b_{0}+b_{1} \cdot I R E G_{t-2}^{j}+b_{2} \cdot B U D_{t-2}+b_{3} \cdot(1-D) \cdot I D E O L R E G_{t-4}
$$

$$
+b_{4} \cdot D \cdot W W A_{t-2}+b_{5} \cdot \operatorname{COST} T_{t-2}+\varepsilon_{t} \cdot
$$

Diese Funktion für das $j$-te Regierungsinstrument enthält neben den Konstanten, dem Instrumenteneinsatz der Vorperiode ${ }^{3)}$ und der Ideologie der Regierung (IDEOLREG) noch ein Maß für die aufgrund eines Popularitätsdefizits der Regierung notwendigen wahltaktischen fi-

1) Die Berücksichtigung des Instrumenteneinsatzes der Vorperiode soll die Hypothese zum Ausdruck bringen, daß Zentralbankmitglieder Bürokraten sind, die sich gegen plötzliche Veränderungen sträuben. Vgl. Frey/Schneider (1978b), S. 6 sowie Schneider (1979), S. 478. Vgl. auch Basler (1979), S. 73, der in diesem Zusammenhang von einer Politik "in kleinen Schritten" spricht.

2) Vgl. Schneider (1979), S. 478

3) Die Berücksichtigung des Instrumenteneinsatzes der Vorperiode soll den EinfluB der Bürokratie zum Ausdruck bringen, von der angenommen wird, daß sie sich allzu großen und abrupten Anderungen des Instrumentene insatzes widersetzen wird. Vgl. Schneider (1979), S. 480 
nanzpolitischen MaBnahmen (WWA ${ }^{1}$ ) sowie das Budgetdefizit des Staates $(B \cup D)^{2)}$ und Kostenfaktoren (COST), die den Instrumenteneinsatz beeinflussen ${ }^{3)}$. Die Frage, ob die Ideologie der Regierung oder die GröBe WWA zum Zuge kommt, wird durch eine Dummy-Variable D bestimmt, welche im Falle eines Popularitätsüberschusses den Wert 0 , im Falle eines Popularitätsdefizits den Wert 1 annimmt ${ }^{4}$ ).

Schließlich enthalten die Modelle eine Popularitätsfunktion, anhand derer der Einfluß wirtschaftlicher Variablen auf die Regierungspopularität gemessen wird ${ }^{5}$ ).

Somit sind in den Modelien von FREY und SCHNEIDER wesentliche Elemente eines politisch-ökonomischen Gesamtmodells enthalten, dessen Struktur aus Abbildung 8 deutlich wird (siehe S. 134).

Die Modelle sind allerdings nicht geschlossen, da die Wirkung der Regierungs - bzw. Zentralbankinstrumente auf die GröBen, anhand derer ihre Wirkungsrichtung gemessen wird $\left(B^{a}-B^{n} b z w \cdot L_{t}-L_{t-1}\right)$, im Modell nicht berücksichtigt ist. Lediglich der Zusammenhang zwischen dem Instrumenteneinsatz der Regierung und den Wählerpräferen-

1) WWA ist ein MaB für die Wiederwahlanstrengungen der Regierung. Dabei wird angenommen, daß der Popularitätssaldo [POP*-POP(t-2)], im Mode 11 von Frey/ Schneider der quadrierte Popularitätssaldo, den Instrumentene insatz bestimmt. Vg1. Schneider (1979), S. 481 und Frey/Schneider (1978b), S. 9. POP* ist der Popularitätsprozentsatz, den die Regierung für die Sicherung der Wiederwahl als ausreichend ansieht. Er wird von den Autoren mit $52 \%$ angenommen. $\mathrm{Vgl}$. Frey/Schneider (1978b), S. 8 und Schneider (1979), S. 479

2) Im Modell von Frey/Schneider werden anstelle des Budgetsaldos die Kredite der Zentralbank an den Staat berücksichtigt. Vgl. Frey/Schneider (1978b), S. 8

3) Als Kostenfaktoren werden Steigerungen des Preisniveaus und der Lohnsätze berücksichtigt. Vgl. Frey/Schneider (1978b), S. 16 sowie Schneider (1979), S. 480 , der lediglich Lohnsteigerungen berücksichtigt.

4) Vg1. Schneider (1979), S. 481 sowie Frey/Schneider (1978b), S. 8 f.

5) Die Verwendung von Popularitätsfunktionen in politometrischen Modellen ist nicht unumstritten. Vgl. zur diesbezüglichen Kontroverse vor allem Dinkel (1977), S. 113 - 118 und S. 129 - 131, Dinkel (1978), Rattinger (1980), S. 53 56 sowie Kirchgäßner/Schneider (1979), S. $91 \mathrm{ff}$. und die Ausführungen in Abschnitt III.2. 


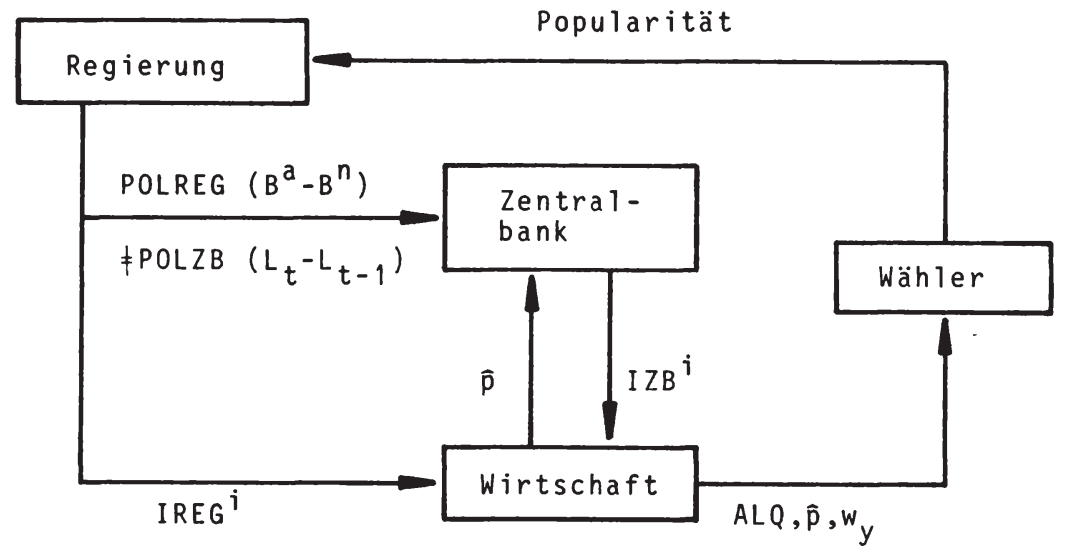

Abbildung 8: Die Interdependenzen im Konfliktmodell von Frey und Schneider ${ }^{1}$ )

zen (der Popularitätsfunktion) läßt sich aus dem Gleichungssystem deduzieren.

Trotz dieses Einwands stellt der Ansatz von FREY und SCHNEIDER einen wichtigen Fortschritt in den Bemühungen um eine positive Analyse des Zentralbankverhaltens dar, da explizit auf bisher nicht berücksichtigte Determinanten dieses Verhaltens, nämlich auf potentielle Konflikte zwischen Regierung und Zentralbank abgestellt wird. Einige Bemerkungen zu den von den Autoren für das Zentralbankverhalten ermittelten Ergebnissen scheinen daher lohnend ${ }^{2}$.

Beide Arbeiten untersuchen die Instrumente Mindestreservesatz-, Diskontsatz und Lombardsatz, wobei sich die Untersuchung SCHNEIDERs

1) Vgl. auch Schneider (1979), S. 483 und Frey/Schneider (1978b), S. 11. Die Abkürzungen bedeuten: POLZB = Politik der Zentralbank (gemessen an der Veränderung der freien Liquiditätsreserven), $A L Q=$ Arbeitslosenquote, $\hat{p}=$ Preissteigerungsrate, $w_{y}=$ Wachstumsrate des realen Bruttosozialprodukts.

2) Vgl. zu den übrigen Schätzergebnissen (Regierungsverhalten, Popularitätsfunktion) Schneider (1979), S. 486 und S. 488 sowie Frey/Schneider (1978b), S. 17 und S. 21. 
auf den Zeitraum 1970 I bis 1977 IV, die Analyse von FREY/SCHNEIDER auf die Zeit von 1957 II bis 1975 IV bezieht ${ }^{1)}$. Bei SCHNEIDER wird zusätzlich der Verkaufssatz für Schatzwechsel mit einer Laufzeit von 60 bis 90 Tagen berücksichtigt ${ }^{2}$ ), bei FREY/SCHNEIDER kommen als weitere abhängige Variable ein nicht näher spezifizierter Satz der Deutschen Bundesbank bei Offenmarktgeschäften sowie Kredite der Bundesbank an den Staat ${ }^{3)}$ hinzu.

Alle geschätzten Parameter $a_{i}$ sind statistisch signifikant von Null verschieden ${ }^{4}$ ) und haben die erwarteten Vorzeichen. So wird die Deutsche Bundesbank den Mindestreservesatz sowie den Diskont- und Lombardsatz im Konfliktfall senken, während sie die Sätze im Falle des Nicht-Konflikts gemäß der Inflationsrate erhöhen wird ${ }^{5)}$. Die Kredite der Zentralbank an den Staat werden demgegenüber im Konfliktfall erhöht, im Fall des Nicht-Konflikts gesenkt ${ }^{6}$ ). Den Haupteinfluß übt jedoch der Instrumenteneinsatz der Vorperiode aus ${ }^{7)}$, was die These einer "Politik in kleinen Schritten" unterstützt.

Die Ergebnisse der Modelle von FREY und SCHNEIDER sprechen also für die These, die Bundesbank richte ihre Politik im Konfliktfall an der Regierungspolitik aus und lassen somit erste Zweifel an der Defacto-Unabhängigkeit der Deutschen Bundesbank aufkommen. Diese Zweifel sind jedoch angesichts einiger Bemerkungen zu den Modellannahmen wieder zu relativieren: Zunächst ist zu beachten, daß die Ergebnisse stark durch die Größen beeinflußt werden, anhand derer auf die expansive bzw. kontraktive Politik der jeweiligen Instanz

1) Die römischen Zahlen hinter den Jahreszahlen kennzeichnen das jeweilige Quartal.

2) Vgl. Schneider (1979), S. 484

3) Vgl. Frey/Schneider (1978b), S. 13

4) Die Irrtumswahrscheinlichkeit beträgt $1 \%$.

5) Vgl. Schneider (1979), S. 484 sowie Frey/Schneider (1978b), S. 19 f.

6) Vgl. Frey/Schneider (1978b), S. 14 und S. $19 \mathrm{f}$.

7) Vgl. ebenda und Schneider (1979), S. 484 
geschlossen wird. Vor allem die Verwendung der Differenz des aktuellen und des konjunkturneutralen Budgetsaldos als MaB für die Richtung der Fiskalpolitik muB angesichts der Probleme bei der Bestimmung des konjunkturneutralen Budgetsaldos als möglicher Kritikpunkt zumindest erwähnt werden ${ }^{1)}$. Weiterhin beinhalten die Modellgleichungen des Zentralbankverhaltens lediglich das Ziel der Preisniveaustabilität ${ }^{2}$ ), während andere, von der Bundesbank möglicherweise ebenfalls verfolgte Ziele, wie z.B. das Zahlungsbilanzziel, unberücksichtigt bleiben ${ }^{3)}$. Störungen anderer Ziele können jedoch den Instrumenteneinsatz der Zentralbank maßgeblich beeinflussen und müßten daher als unabhängige Variable berücksichtigt werden. Die Tatsache, daß dies nicht geschehen ist, muß daher bei der Interpretation der Ergebnisse von FREY und SCHNEIDER zur Vorsicht mahnen. Vor allem aber die von den Autoren verwendete Konfliktdefinition muB zur Kritik herausfordern. Ein Konflikt zwischen Regierung. und Bundesbank, der letztere aus Furcht vor dem Verlust ihrer unabhängigen stellung ${ }^{4)}$ zu einer Richtungsänderung in ihrer Geldpolitik veranlaBt, ist sicherlich nicht schon dann gegeben, wenn die Veränderung der freien Liquiditätsreserven und die Differenz zwischen aktuellem und konjunkturneutralem Budgetsaldo eine unterschiedliche Wirkungsrichtung geld- und finanzpolitischer Meßgrößen anzeigen. Dies gilt insbesondere in einem Staat wie der Bundesrepublik Deutschland, in dem die offentlichkeit sehr sensibel auf eine Einschränkung der Zentralbankautonomie reagieren würde. Die Bundesbank wird deshalb nicht schon dann eine Einschränkung ihrer Unabhängigkeit befürchten müssen, wenn aufgrund zweier Meßgrößen, deren Eignung als Indikator für die Wirkungsrichtung wirtschaftspolitischer Instrumente zumindest umstritten ist, eine entgegengerichtete Wirkung geld-und finanzpolitischer Instrumente festgestel1t wird.

1) Vgl. zur Berechnung des konjunkturellen Effekts des öffentlichen Haushalts mittels des Konzepts des Sachverständigenrats vor allem Scherer (1977), S. $99 \mathrm{ff}$.

2) Vgl. die Gleichung (V.9) und Gleichung (V.10).

3) Vgl. Schneider (1979), S. 476, Fußnote 10 sowie Gäfgen (1979), S. 500

4) Vgl. Frey/Schneider (1978b), S. 4 
Ein Konflikt wird äuBerstenfalls dann eintreten, wenn Regierung und Zentralbank unterschiedliche Zielprioritäten haben und die von beiden Institutionen präferierten Ziele gleichzeitig in Gefahr sind. Im folgenden Abschnitt wird gezeigt, daB eine in dieser Weise definierte Konfliktsituation zu Ergebnissen fuhrt, die zu einer Ablehnung der These von der De-facto-Abhängigkeit der Deutschen Bundesbank führen.

\subsection{Ein Satisfizierungsmodell zur Beurteilung der Bundes-} bankautonomie

Das im folgenden vorgestellte Konfliktmodell geht wie die Modelle von FREY/SCHNEIDER und SCHNEIDER davon aus, daB die Wahrung der Preisniveaustabilität oberstes Ziel ("Ideologie") der Bundesbank ist. Dabei wird angenommen, die Bundesbank strebe an, die aktuelle Preissteigerungsrate $\hat{\mathrm{p}}$ nicht über einen Wunschwert $\hat{\mathrm{p}}$ ansteigen $z u$ lassen. Der Einfluß der Regierung auf die Bundesbankpolitik wird allerdings nicht mehr durch den Saldo des aktuellen und konjunkturneutralen Budgetsaldos bestimmt, sondern es wird unterstellt, daB die Regierung primär an der Vermeidung von Arbeitslosigkeit interessiert ist und im Falle einer Gefährdung beider Ziele das Ziel der Preisniveaustabilität hintanstellt ${ }^{1}$ ). GemäB der am Ende des letzten Abschnitts erwähnten Konfliktdefinition ergibt sich daher ein Konflikt zwischen Regierung und Bundesbank, wenn die Arbeitslosenquote ALQ über ihrem Wunschwert ALQ* und gleichzeitig die Preissteigerungsrate $\hat{\mathrm{p}}$ über ihrem Wunschwert $\hat{\mathrm{p}}^{\star}$ liegt, d.h. wenn $g i 1 t^{2}$ )

$A_{t-1}>A L Q_{t-1}^{\star} \quad$ und $\quad \hat{p}_{t-1}>\hat{p}_{t-1}^{\star} \cdot$

1) Diese Annahme scheint für den empirisch untersuchten Zeitraum von 1969 III bis 1980 II nicht unplausibel, da für sozialdemokratische Regierungen geme inhin eine Favorisierung des Beschäftigungsziels unterstellt wird. Vgl. Kirschen, Benard, Besters, u.a. (1964), S. 227

2) Bei der Ermittlung der Zielabweichungen wird ein Erkennungs-lag von einer Periode (1 Quartal) unterstellt. 
Liegt eine der beiden Zielgrößen unter ihrem Wunschwert oder wird der Wunschwert gerade erreicht, so liegt die Situation des NichtKonflikts vor. Die These von der Abhängigkeit der Zentralbank würde in diesem Ansatz offensichtlich dann bestätigt, wenn die Bundesbank ihren Instrumenteneinsatz im Konfliktfall an der Abweichung der Arbeitslosenquote, im Falle des Nicht-Konflikts an der Abweichung der Preissteigerungsrate ausrichten würde. Zur Oberprüfung der Abhängigkeitsthese wird die folgende Modellgleichung aufgestellt ${ }^{1) 2}$ ):

$$
I Z B_{t}^{i}=c_{0}+C_{1} \cdot I Z B_{t-1}^{i}+C_{2} \cdot E_{t-1} \cdot\left(A L Q-A L Q^{*}\right)_{t-\tau}
$$

$$
+c_{3} \cdot\left(1-E_{t-1}\right) \cdot\left(\hat{p}-\hat{p}^{\star}\right)_{t-\tau}+\theta_{t} \cdot
$$

Wie in den Modellen von FREY und SCHNEIDER wird der Instrumenteneinsatz der Vorperiode als Determinante des Instrumenteneinsatzes der laufenden Periode mitberücksichtigt. E $t$ ist eine Dummy-Variab$1 e^{3)}$, die im Konfliktfall den Wert 1, im Falle des Nicht-Konflikts den Wert 0 annimmt. Es wird angenommen, daB die Zentralbank den Instrumenteneinsatz anhand der Zielabweichungen der Vorperioden bestimmt, wobei im Fall der Abweichung der Preissteigerungsrate ein Lag von zwei Quartalen, im Falle der Abweichung der Arbeitslosenquote ein Lag von einem bzw. zwei Quartalen unterstellt wird. Die Wunschwerte entstammen dabei dem jeweiligen Jahreswirtschaftsbericht der Bundesregierung ${ }^{4}$ ).

1) Auf eine Normierung der Variablen wurde verzichtet, da sich die Standardabweichungen nur geringfügig unterscheiden.

2) $\mathrm{Vgl}$. zur Verwendung von Zielabweichungen in Reaktionsfunktionen der Zentralbank auch Froyen (1974).

3) Der Zeitindex t-1 der Dummy-Variablen bringt den unterstellten ErkennungsLag von einem Quartal zum Ausdruck.

4) Für den Wunschwert der Preissteigerungsrate dürfte dieser Wert sicherlich mit Ungenauigkeiten behaftet sein, da der Wunschwert der Regierung nicht mit dem der Zentralbank übereinzustimmen braucht. In Ermangelung geeigneter Quellen für Wunschwerte der Zentralbank werden daher die Wunschwerte der Regierung a)s Approximation der Wunschwerte der Bundesbank verwendet. Dieser Vorgehensweise bedient sich auch Basler (1979), S. $76 \mathrm{f}$. 
Im folgenden wird lediglich das Instrument des Diskontsatzes ${ }^{D}$ exemplarisch untersucht ${ }^{1}$, so daB für die koeffizienten $c_{1}$ und $c_{3}$ ein positives, für $c_{2}$ ein negatives Vorzeichen erwartet wird. Tabelle 10 auf Seite 140 gibt die OLS-Schätzergebnisse für den Zeitraum 1969 III - 1980 II bzw. 1973 I - 1980 II wieder ${ }^{2}$.

Aus den geschätzten Gleichungen (V.12.1) bis (V.12.3) ist ersichtlich, daB die geschätzten Koeffizienten die erwarteten Vorzeichen haben, die Abweichung der Arbeitslosenquote im Konfliktfall jedoch keinen statistisch signifikanten EinfluB ausibt ${ }^{3}$ ). Im übrigen deutet der im Falle der Verwendung endogen verzögerter Variabler zum Test auf Autokorrelation anzuwendende h-Test auf Autokorrelation hin. Durch Bilden der verallgemeinerten ersten Differenzen entsprechend der COCHRANE-ORCUTT-Methode läBt sich jedoch eine Variablentransformation und auf dieser Basis eine neve Schätzung durchführen ${ }^{4)}$, deren Störvariable nicht mehr autokorreliert sind. Die für die Zeit von 1969 IV - 1980 II durchgeführten Schätzungen sind aus den Gleichungen (V.12.4) und (V.12.5) der Tabelle 10 ersicht'ich und unterscheiden sich von den in den bisherigen Gleichungen ermittelten Ergebnissen nicht grundsätzlich. Der Erklärungsanteil sinkt jedoch von $86 \%$ auf $75 \%$ der Varianz. Welche Schlubfolgerungen lassen sich aufgrund dieser Ergebnisse ziehen? Da die Abweichung der Arbeitslosenquote vom Zielwert im Konfliktfall keinen

1) Für den Lombardsatz lassen sich ähnliche Ergebnisse ermitteln.

2) Datenbasis: Es wurden Quartalswerte verwendet, wobei im Falle von Preissteigerungsrate und Arbeitslosenquote auf saisonbereinigte Werte zurückgegriffen wurde, da anzunehmen ist, daß saisonbedingte Anderungen der Zielgrößen für Zentralbankentscheidungen keine entscheidende Rolle spielen. Datenquellen: Monatsberichte der Deutschen Bundesbank; Statistische Beihefte zu den Monatsberichten der Deutschen Bundesbank, Reihe 4, saisonbere inigte Wirtschaftszahlen. (Der Berechnung der Preissteigerungsrate wurde der Preisindex für die Lebenshaltung aller privaten Haushalte zugrundegelegt.)

3) Die OLS-Schätzergebnisse können bei Verwendung endogen verzögerter Variablen Verzerrungen bei den geschätzten Koeffizienten und den F-Werten aufweisen. Bei Verwendung der ersten Differenzen der Instrumente als abhängige Variable ergeben sich jedoch keine Anderungen hinsichtlich der Vorzeichen und der Signifikanz.

4) Vgl. Z.B. Bamberg/Schittko (1979), S. 65 f. 


\begin{tabular}{|c|c|c|c|c|c|c|c|c|}
\hline \multirow{2}{*}{$\begin{array}{l}\text { Gle ichungs- } \\
\text { nummer } \\
\text { (Zeitraum) }\end{array}$} & \multirow{2}{*}{$\begin{array}{l}\text { Endogen } \\
\text { verzögerte } \\
\text { Variable }\end{array}$} & \multirow{2}{*}{$E \cdot\left(A L Q-A L Q^{\star}\right)_{t-\tau}$} & \multirow[t]{2}{*}{$\tau$} & \multirow{2}{*}{$(1-E) \cdot\left(\hat{p}-\hat{p}^{*}\right)_{t-2}$} & \multirow{2}{*}{$\begin{array}{l}\text { Absolut- } \\
\text { glied }\end{array}$} & \multicolumn{3}{|c|}{ Teststatistiken } \\
\hline & & & & & & $R^{2}$ & $h$ & $\mathrm{FG}$ \\
\hline $\begin{array}{l}(V .12 .1) \\
(1969 \text { III - } \\
1980 \text { II })\end{array}$ & $\begin{array}{l}0,8529 \star \star \\
(169,320)\end{array}$ & $\begin{array}{r}-0,5453 \\
(2,570)\end{array}$ & 1 & $\begin{array}{l}0,4090 * \star \\
(10,440)\end{array}$ & 0,7890 & 0,86 & 2,90 & 40 \\
\hline $\begin{array}{l}(V .12 .2) \\
(1969 \text { III - } \\
1980 \text { II })\end{array}$ & $\begin{array}{l}0,8431 * \star \\
(155,320)\end{array}$ & $\begin{array}{r}-0,3054 \\
(1,023)\end{array}$ & 2 & $\begin{array}{l}0,4180 * \star \\
\quad(10,496)\end{array}$ & 0,8156 & 0,85 & 2,79 & 40 \\
\hline $\begin{array}{l}(V .12 .3) \\
(1973 \text { I - } \\
1980 \text { II })\end{array}$ & $\begin{array}{l}0,8693^{\star \star} \\
(169,485)\end{array}$ & $\begin{array}{r}-0,3435 \\
(1,958)\end{array}$ & 2 & $\begin{array}{r}0,4621 * * \\
(16,377)\end{array}$ & 0,7840 & 0,91 & 1,68 & 26 \\
\hline $\begin{array}{l}(V .12 .4) \\
(1969 \text { IV - } \\
1980 \text { II })\end{array}$ & $\begin{array}{l}0,7745^{\star \star} \\
(84,526)\end{array}$ & $\begin{array}{r}-0,4925 \\
(3,007)\end{array}$ & 1 & $\begin{array}{r}0,3928 * \star \\
(9,716)\end{array}$ & 0,6864 & 0,75 & $-0,74$ & 39 \\
\hline $\begin{array}{l}(V .12 .5) \\
(1969 \text { IV - } \\
1980 \text { II })\end{array}$ & $\begin{array}{l}0,7726 * \star \\
(81,887)\end{array}$ & $\begin{array}{l}-0,2430 \\
(0,894)\end{array}$ & 2 & $\begin{array}{r}0,3929 * \star \\
(9,240)\end{array}$ & 0,6985 & 0,75 & $-0,71$ & 39 \\
\hline
\end{tabular}

Tabelle 10: Diskontpolitik der Deutschen Bundesbank. Test der Abhängigkeitsthese. OLSSchätzungen mit Quartalsdaten für die Perioden 1969 III - 1980 II und 1973 I -1980 II $\left.^{1}\right)$

1) Die unterhalb der geschätzten Koeffizienten aufgeführten Klammerwerte geben die F-Statistik an. Zwei Sterne hinter dem jeweiligen Koeffizienten bedeuten, daß die Variable auf dem $99 \%-N i v e a u$ einen statistisch signifikanten Einfluß hat. FG bezeichnet die Anzahl der Freiheitsgrade. Unterhalb der Gleichungsnummer ist der entsprechende Schätzzeitraum aufgeführt. 
signifikanten EinfluB auf Diskontsatzvariationen ausuibt, kann die These der De-facto-Abhängigkeit der Deutschen Bundesbank nicht bestätigt werden. Als empirisch abgesichert kann lediglich der EinfluB der endogen verzögerten Variablen und der Abweichung der Preissteigerungsrate vom Zielwert im Falle des Nicht-Konflikts gelten.

Fraglich bleibt jedoch, wie sich die Bundesbank in Konfliktfällen verhalten hat. Hätte sie in Phasen, in denen beide Ziele gefährdet waren, strikt das Ziel der Preisniveaustabilität verfolgt, so müBte sich ein statistisch signifikanter EinfluB der Abweichung der Preissteigerungsrate im Konfliktfall nachweisen lassen. Die Hypothese einer strikten Verfolgung des Ziels der Preisniveaustabilität läBt sich somit durch Schätzung der Koeffizienten der Gleichung

$$
i_{t}^{D}=d_{0}+d_{1} \cdot i_{t-1}^{D}+d_{2} \cdot E_{t-1} \cdot\left(\hat{p}-\hat{p}^{\star}\right) t-\tau
$$

$$
+d_{3} \cdot\left(1-E_{t-1}\right) \cdot\left(A L Q-A L Q^{*}\right)_{t-\tau}+\rho_{t}
$$

überprüfen. Für den Zeitraum von 1973 I - 1980 II ergeben sich die Koeffizienten $d_{i}$ gemäB Gleichung $(V .13 .1)^{1)}$ :

$(v .13 .1)$

$$
i_{t}^{D}=0,4487+\underset{(i 77,319)}{0,9509} \cdot i_{t-1}^{D}+\underset{(0,000)}{0,0} \dot{E}_{t-1} \cdot\left(\hat{p}-\hat{p}^{*}\right)_{t-2}
$$

$$
-\underset{(6,486)}{0,4412} \cdot\left(1-E_{t-1}\right) \cdot\left(A L Q-A L Q^{*}\right)_{t-1} \cdot
$$

(Teststatistiken: $R^{2}=0,88 ; h=1,18 ; F G=27$. )

Es wird deutlich, daB in dieser Gleichung die endogen verzögerte Variable und die Abweichung der Arbeitslosenquote im Nicht-Konfliktfall einen statistisch signifikanten EinfluB aufweisen ${ }^{2}$, die $A b-$

1) Die jeweils in Klammer gesetzten Zahlen geben die F-Werte wieder.

2) Das Signifikanzniveau beträgt im Fall der endogen verzögerten Variablen $99 \%$, im Falle von (ALQ-ALQ*) $95 \%$. Für die um 2 Perioden verzögerte Zielabweichung konnte allerdings kein statistisch signifikanter EinfluB nachgewiesen werden. 
weichung der Preissteigerungsrate im Konfliktfall jedoch nicht. Offensichtlich hat sich die Deutsche Bundesbank im Untersuchungszeitraum nicht systematisch in dem Sinne verhalten, daß sie immer nur konsequent einem Ziel den Vorrang eingeräumt hat ${ }^{1}$ ). Vielmehr scheint eher die Hypothese zuzutreffen, die BASLER auf der Grundlage der Ergebnisse seines Optimierungsansatzes aufgestellt hat: Danach ist "offenkundig, daB die Deutsche Bundesbank kein starres Präferenzschema hatte, sondern im allgemeinen jenem Ziel Vorrang gab, das am stärksten gestört war"2). Für den von ihm betrachteten Zeitraum von 1958 I - 1974 I stellt BASLER überdies fest, daß "die deutschen Geldpolitiker der Preisstabilität insgesamt gesehen die größte Bedeutung beimaßen" 3 ).

Die zuerst genannte Hypothese $1 \ddot{B}$ t sich auch anhand des in dieser Arbeit vorgestellten Satisfizierungsmodells testen, indem folgende Modellgleichungen zugrundegelegt werden:

$$
\text { (V.14) } \quad i_{t}^{D}=e_{0}+e_{1} \cdot i_{t-1}^{D}+e_{2} \cdot F_{t-\tau} \cdot\left(\hat{p}-\hat{p}^{\star}\right){ }_{t-\tau}+e_{3} \cdot G_{t-\tau} \cdot\left(A L Q-A L Q^{\star}\right)_{t-\tau}+\alpha_{t} \cdot
$$

Die Dummy-Variable $F_{t}$ nimmt immer dann, wenn das Ziel der Preisniveaustabilität stärker gefährdet ist ${ }^{4}$, den Wert 1 an. Ist das Beschäftigungsziel stärker gefährdet, so ist $F_{t}=0 . G_{t}$ ist dagegen

1) Dies gilt auch, weil ein Einfluß der Abweichung eines Ziels im Nicht-Konfliktfall noch nicht bedeutet, daß die Bundesbank das jeweils andere Ziel in diesen Phasen nicht verfolgte. Ein Blick auf das verwendete Datenmaterial (vgl. Anhang, Punkt 2.) macht nämlich deutlich, daß im Untersuchungszeitraum Phasen des Nicht-Konflikts auftraten, in denen das Ziel der Preisniveaustabilität (Beschäftigungsziel) nicht gefährdet war, so daß sich eine negative Differenz $\left(z-z^{\star}\right)$ einstellte. Ein positiver Koeffizient vor dem Ausdruck $(1-E) \cdot\left(\hat{p}-\hat{p}^{\star}\right)$ bzw. ein negativer Koeffizient vor dem Ausdruck $(1-E) \cdot\left(A L Q-A L Q^{*}\right)$ bedeutet dann, da $B$ in diesen Situationen eine Diskontsatzsenkung (Diskontsatzerhöhung) stattgefunden hat, m.a.W. daß das Beschäftigungsziel (Ziel der Preisniveaustabilität) verfolgt wurde.

2) Basler (1979), S. 90. "Deutsche Bundesbank" im Original mit DBk abgekürzt.

3) Ebenda

4) Das AusmaB der Gefährdung eines Ziels wird anhand der Abweichung vom Zielwert bestimmt. 
gleich 1, wenn das Beschäftigungsziel stärker gefährdet ist. Für $\hat{p}_{t}>\hat{p}_{t}{ }^{*}$ ist $G_{t}=0$. Ist keines der beiden Ziele gefährdet, so ist $G_{t}=F_{t}=0$. Die Schätzergebnisse sind in Tabelle 11 zusammengefaBt.

In zwe $i$ der vier aufgeführten Schätzungen üben beide Zielabweichungen zu Zeiten, in denen die Ziele am stärksten gefährdet sind, einen statistisch signifikanten EinfluB auf Diskontsatzvariationen aus. Nur wenn im Falle von Abweichungen des Beschäftigungsziels eine Reaktionsverzögerung von zwei Quartalen unterstellt wird, läBt sich kein statistisch signifikanter Einfluß des Beschäftigungsziels auf die Diskontpolitik ermitteln.

Offen bleibt allerdings, ob die von BASLER festgestellte Priorität des Ziels der Preisniveaustabilität während des Gesamtzeitraums auch noch für die jüngste Vergangenheit zutrifft. Genau diese Frage kann jedoch nicht beantwortet werden, wenn das Zentralbankverhalten anhand eines Satisfizierungsansatzes beurteilt wird, denn Zielprioritäten wirtschaftspolitischer Instanzen lassen sich nur auf der Basis eines Optimierungsansatzes ermitteln.

Auf der Grundlage des vorgestellten Satisfizierungsmodells und der im Rahmen dieses Ansatzes gewählten Konfliktdefinition läBt sich jedoch zusammenfassend feststellen, daB Abweichungen wirtschaftspolitischer Ziele von ihrem Wunschwert in Phasen des Nicht-Konflikts, also dann, wenn das jeweils andere Ziel nicht gefährdet ist, einen statistisch signifikanten EinfluB auf Diskontsatzvariationen ausüben. Ebenso ist in Zeiten, in denen ein Ziel stärker gefährdet ist als das andere, ein statistisch signifikanter EinfluB des jeweils am stärksten gefährdeten Ziels auf die Diskontpolitik festzustellen ${ }^{1)}$.

1) Dies gilt jedenfalls dann, wenn für Abweichungen des Ziels der Preisniveaustabilität vom Wunschwert eine Reaktionsverzögerung von zwei Quartalen, im Falle von Abweichungen des Beschäftigungsziels ein Lag von einem Quartal unterstellt wird. 


\begin{tabular}{|c|c|c|c|c|c|c|c|c|c|}
\hline \multirow{2}{*}{$\begin{array}{l}\text { Gleichungs- } \\
\text { nummer }\end{array}$} & \multirow{2}{*}{$\begin{array}{l}\text { Endogen } \\
\text { verzögerte } \\
\text { Variable }\end{array}$} & \multirow{2}{*}{$F_{t-\tau} \cdot\left(\hat{p}-\bar{p}^{\star}\right) t-\tau$} & \multirow[t]{2}{*}{$\tau$} & \multirow{2}{*}{$G_{t-\tau} \cdot\left(A L Q-A L Q^{*}\right)_{t-\tau}$} & \multirow[t]{2}{*}{$\tau$} & \multirow{2}{*}{$\begin{array}{l}\text { Absolut- } \\
\text { glied }\end{array}$} & \multicolumn{3}{|c|}{ Teststatistiken } \\
\hline & & & & & & & $R^{2}$ & h & FG \\
\hline (V.14.1) & $\begin{array}{l}0,8663^{\star \star} \\
(157,863)\end{array}$ & $\begin{array}{l}0,4082^{\star} \\
\quad(6,817)\end{array}$ & 1 & $\begin{array}{r}-0,3526^{\star} \\
(4,867)\end{array}$ & 1 & 0,6817 & 0,91 & $-0,74$ & 26 \\
\hline (V.14.2) & $\begin{array}{l}0,7661 \star \star \\
\quad(125,456)\end{array}$ & $\begin{array}{l}0,6573 \star \star \\
(20,237)\end{array}$ & 2 & $\begin{array}{r}-0,1988 \\
(1,853)\end{array}$ & 2 & 0,9662 & 0,93 & 0,72 & 26 \\
\hline (V.14.3) & $\begin{array}{l}0,7901 . \star \star \\
(144,638)\end{array}$ & $\begin{array}{l}0,6012 \star \star \\
(17,186)\end{array}$ & 2 & $\begin{array}{l}-0,2888^{*} \\
(4,272)\end{array}$ & 1 & 0,9156 & 0,93 & 0,84 & 26 \\
\hline$(v .14 .4)$ & $\begin{array}{l}0,8344 \star \star \\
(133,577)\end{array}$ & $\begin{array}{l}0,4836 \star \star \\
(10,067)\end{array}$ & 1 & $\begin{array}{l}-0,2870 \\
(3,154)\end{array}$ & 2 & 0,7707 & 0,91 & $-0,85$ & 26 \\
\hline
\end{tabular}

Tabelle 11: Diskontpolitik der Deutschen Bundesbank. Test auf Verfolgung des jeweils am meisten gefährdeten Ziels. OLS-Schätzung mit Quartalsdaten von 1973 I - 1980 II I)

1) Zwei Sterne hinter dem jeweiligen Koeffizienten bedeuten, daß die Variable auf dem $99 \%-N i v e a u$ einen statistisch signifikanten Einfluß hat. Im Falle eines Sterns beträgt das Signifikanzniveau nur $95 \%$. 
Die Gesamtheit der Ergebnisse läßt daher den SchluB zu, daB

1. zum gegenwärtigen Zeitpunkt keine Anzeichen für eine De-factoAbhängigkeit der Deutschen Bundesbank von der Regierung vorliegen und

2. die Deutsche Bundesbank ihrer gesamtwirtschaftlichen Verantwortung durchaus gerecht geworden ist, indem sie das Beschäftigungsziel immer dann zu erreichen versuchte, wenn das ihr primär vorgegebene Ziel der Preisniveaustabilität nicht gefährdet war bzw. nicht so stark gefährdet war wie das Beschäftigungsziel.

\subsection{Fazit: Die Autonomie der Deutschen Bundesbank}

Selbstverständlich können die mit Hilfe des vorgestellten Satisfizierungsmodells ermittelten Ergebnisse keinen Anspruch auf Allgemeingültigkeit erheben, zumal lediglich ein Instrument der Zentralbank untersucht wurde. Als Ergebnis bleibt jedoch festzuhalten, daB die aus bisherigen empirischen Untersuchungen gezogene SchluBfolgerung der Abhängigkeit der Deutschen Bundesbank nicht zwingend ist. Vielmehr kann auf der Basis einer alternativen - und nach Ansicht des Verfassers realistischeren - Definition des Konflikts zwischen Regierung und Bundesbank auch die gegenteilige These empirisch bestätigt werden.

Vor allem aber macht das gefundene Ergebnis auf die zentrale Bedeutung der Konfliktdefinition in empirischen Analysen der Zentralbankautonomie aufmerksam ${ }^{1)}$. Ein nächster Schritt weiterer Forschung auf diesem Gebiet müßte daher in der Diskussion problemadäquater Definitionen eines Konflikts zwischen Regierung und Zentralbank bestehen. Auf der Basis allgemein akzeptierter Konfliktdefinitionen erscheint eine Weiterentwicklung des vorgestellten Satisfizierungs-

1) Voraussetzung ist selbstverständlich, daß der Analyse ein Modell zugrundegelegt wird, in dem der Zentralbank eine konfliktvermeidende Satisfizierungsstrategie unterstellt wird. 
modells - etwa durch Berücksichtigung weiterer Ziele und Instrumente - notwendig und sinnvoll.

Insgesamt gesehen erscheinen die bisher vorliegenden empirischen Ergebnisse noch nicht überzeugend genug, um die auf der Basis der institutionellen Analyse aufgestellte Hypothese der De-facto-Unabhängigkeit der Deutschen Bundesbank zu widerlegen.

2.2.2.2. Empirische Analyse der Geldmengen- und Inflationswirkungen staatlicher Budgetdefizite in den USA und in der Bundesrepublik Deutschland

\subsection{Vorgehenswe ise}

Die bisherigen Ausführungen legen den Schluß nahe, daß Budgetdefizite in den USA zu Steigerungen im Geldmengenwachstum führen, während in der Bundesrepublik Deutschland nicht oder in geringerem Maße mit dieser Entwicklung zu rechnen ist. Diese These wird im folgenden empirisch überprüft. Zunächst werden einige empirische Untersuchungen des Zusammenhangs zwischen staatlichen Budgetsalden und Geldmenge in den USA diskutiert, wobe $i$ das Hauptinteresse auf Arbeiten von BARRO ${ }^{1)}$, HAMBURGER/ZWICK ${ }^{2}$ ) und MCMILLIN/BEARD ${ }^{3}$ ) gelegt wird. Auf der Basis der Diskussion dieser Ansätze wird dann eine eigene Schätzung durchgeführt. Die dabei verwendeten Modellgleichungen werden dann im folgenden Abschnitt für die Bundesrepublik Deutschland getestet, um einen sinnvollen Vergleich vornehmen zu können. Durch den Vergleich der Schätzergebnisse wird die eingangs formulierte These bestätigt.

1) Vgl. Barro (1978) und (1978a)

2) Vgl. Hamburger/Zwick (1981)

3) Vgl. McMill in/Beard (1980) 
2.2.2.2.2. Budgetdefizite, Geldmengenänderungen und Inflationsrate in den USA

2.2.2.2.2.1. Die historische Entwicklung der Budgetsalden und der Geldmenge in den USA - einige Bemerkungen zur empirischen Analyse von BUCHANAN/WAGNER

Zur empirischen Unterstützung ihrer Hypothese betrachten BW während des Zeitraums zwischen 1947 und 1974, dessen zweite Hälfte (1961 1974) sie als "keynesianische Periode"1) bezeichnen, die Entwicklung des zentralstaatlichen Budgetsaldos, der Geldmenge und des $\mathrm{Be}-$ stands an staatlichen Wertpapieren im Portefeuille des Federal Reserve system.

Tabelle 12 ist zu entnehmen, daB in der Zeit zwischen 1947 und 1960 fünf OberschuBjahre auftraten, in denen die Wachstumsrate der Geldmenge relativ niedrig war. In den restlichen neun Defizitperioden übertraf die Geldmengenwachstumsrate die durchschnittliche Wachstumsrate der betrachteten Vierzehn-Jahres-Periode. Letztere wird durch die durchschnittliche Wachstumsrate der Geldmenge im Zeitraum 1961 - 1974 um drei Prozentpunkte übertroffen. Die durchschnittliche Wachstumsrate der Geldmenge war daher in der "keynesianischen Periode", die durch Budgetdefizite gekennzeichnet war, deutlich höher als in der Zeit von $1947-1960$.

Oberdies macht ein Blick auf die Veränderung des Bestands an Staatstiteln im Portefeuille des Federal Reserve System nach Ansicht von $B W$ deutlich, daß die Zentralbank eine Hauptfinanzierungsquelle für statliche Budgetdefizite sei. Die Tatsache gelte für den gesamten betrachteten Zeitraum, werde aber vor allem in der "keynesianischen Periode", die durch wachsende Budgetdefizite ohne kompensierende Budgetüberschüsse gekennzeichnet war, relevant ${ }^{2}$. Die These

1) Vgl. Buchanan/Wagner (1977), S. 115

2) $\mathrm{Vgl}$. ebenda, S. $115 \mathrm{f}$. 


\begin{tabular}{|c|c|c|c|c|c|c|}
\hline Jahr & $D E F$ & $\begin{array}{c}\triangle B I L L S \\
F E D\end{array}$ & $M_{1}$ & $P$ & $y^{r}$ & $A L Q$ \\
\hline $\begin{array}{l}1947 \\
1948 \\
1949 \\
1950 \\
1951 \\
1952 \\
1953 \\
1954 \\
1955 \\
1956 \\
1957 \\
1958 \\
1959 \\
1960 \\
1961 \\
1962 \\
1963 \\
1964 \\
1965 \\
1966 \\
1967 \\
1968 \\
1969 \\
1970 \\
1971 \\
1972 \\
1973 \\
1974\end{array}$ & $\begin{array}{r}-2,4 \\
-3,9 \\
3,6 \\
0,4 \\
3,4 \\
5,8 \\
9,2 \\
3,7 \\
2,8 \\
-3,8 \\
-0,6 \\
7,1 \\
7,1 \\
-1,9 \\
6,3 \\
7,2 \\
6,7 \\
8,2 \\
4,7 \\
7,3 \\
14,0 \\
16,1 \\
-7,2 \\
10,5 \\
24,8 \\
17,3 \\
7,9 \\
10,9\end{array}$ & $\begin{array}{r}-0,7 \\
0,7 \\
-4,4 \\
1,9 \\
3,0 \\
0,9 \\
1,2 \\
-1,0 \\
-0,1 \\
0,1 \\
-0,7 \\
2,1 \\
0,3 \\
0,8 \\
1,5 \\
1,9 \\
2,8 \\
3,4 \\
3,8 \\
3,5 \\
4,8 \\
3,8 \\
4,3 \\
4,9 \\
8,1 \\
-0,3 \\
8,6 \\
2,0\end{array}$ & $\begin{array}{l}111,8 \\
112,3 \\
111,2 \\
114,1 \\
119,2 \\
125,2 \\
128,3 \\
130,3 \\
134,4 \\
136,0 \\
136,7 \\
138,4 \\
143,6 \\
143,5 \\
146,5 \\
149,7 \\
154,1 \\
160,2 \\
167,1 \\
174,7 \\
181,5 \\
194,3 \\
206,4 \\
214,5 \\
228,9 \\
245,0 \\
263,3 \\
277,7\end{array}$ & $\begin{array}{r}49,70 \\
53,13 \\
52,59 \\
53,64 \\
57,27 \\
58,00 \\
58,88 \\
59,69 \\
60,98 \\
62,90 \\
65,02 \\
66,06 \\
67,52 \\
68,67 \\
69,28 \\
70,55 \\
71,59 \\
72,71 \\
74,32 \\
76,76 \\
79,02 \\
82,57 \\
86,72 \\
91,36 \\
96,02 \\
100,00 \\
105,80 \\
116,02\end{array}$ & $\begin{array}{r}468,3 \\
487,7 \\
490,7 \\
533,5 \\
576,5 \\
598,5 \\
621,8 \\
613,7 \\
654,8 \\
668,8 \\
680,9 \\
679,5 \\
720,4 \\
736,8 \\
755,3 \\
799,1 \\
830,7 \\
874,4 \\
925,9 \\
981,0 \\
1007,7 \\
1051,8 \\
1078,8 \\
1075,3 \\
1107,5 \\
1171,1 \\
1235,0 \\
1217,8\end{array}$ & $\begin{array}{l}3,9 \\
3,8 \\
5,9 \\
5,3 \\
3,3 \\
3,0 \\
2,9 \\
5,5 \\
4,4 \\
4,1 \\
4,3 \\
6,8 \\
5,5 \\
5,5 \\
6,7 \\
5,5 \\
5,7 \\
5,2 \\
4,5 \\
3,8 \\
3,8 \\
3,6 \\
3,5 \\
4,9 \\
5,9 \\
5,6 \\
4,9 \\
5,6\end{array}$ \\
\hline
\end{tabular}

Tabelle 12: Budgetdefizite der Zentralregierung (DEF), Anderung des Bestands an Statspapieren im Portefeuille der Zentralbank ( $\triangle B I L L S F E D)$, Geldmenge $\left(M_{1}\right)$, reales Sozialprodukt $\left(Y^{r}\right)$, jeweils in Mrd. \$, Preisindex des Bruttosozialprodukts ( $P$ ) und Arbeitslosenquote ( $A L Q$ ) in den USA. Zeitreihen von 1947 - 1974 ${ }^{1}$ )

1) Datenquellen: Buchanan/Wagner (1977), S. 115, die sich auf verschiedene Ausgaben des Federal Reserve Bulletin beziehen, Gordon, R.J. (1978), Appendix B, S. XV ff. 
von $B W$ wird durch eine Untersuchung von $\mathrm{ROESCH}^{1}$ ) unterstützt. ROESCH stellt fest, daB die amerikanische Staatsschuld zwischen 1952 und dem 3. Quartal 1961 lediglich mit einer durchschnittlichen Rate von 1 \% zugenommen hat. Gleichzeitig nahmen die statsschuldtitel im Besitz des Federal Reserve System im Jahresdurchschnitt um $2 \%$ zu. Zwischen dem letzten Quartal 1961 und Mitte 1975 stieg die Staatsschuld dagegen mit einer durchschnittlichen Rate von $3,5 \%$ und die Zentralbank erhöhte ihren Bestand an Staatspapieren um durchschnittlich 8,5 \% im Jahr. Eine hierzu parallele Entwicklung war beim Wachstum der Geldmenge und der Geldbasis festzustellen. So nahm die Geldmenge bis zum 3. Quartal 1961 um durchschnittlich 1,6\% jährlich zu. Von Ende 1961 bis Mitte 1975 betrugen die mittleren Zuwachsraten von Geldmenge und Geldbasis dagegen 5, 1 \% bzw. $5,7 \%$. Auch ROESCH kommt daher zu dem Ergebnis: "In the past, deficits have created pressures for increased money supply growth - the greater the deficit, the greater have been the pressures on the monetary authorities for monetary expansion ${ }^{2}$ )."

Die Frage, ob Budgetdefizite als Hauptursache der monetären Expansion der letzten beiden Jahrzehnte angesehen werden können, läBt sich indessen auf der Basis der Untersuchung von ROESCH nicht endgiultig klären ${ }^{3)}$. Mehr Klarheit können erst ökonometrische Untersuchungen des behaupteten Zusammenhang schaffen, denen wir uns im folgenden Abschnitt zuwenden werden.

2.2.2.2.2.2. Okonometrische Untersuchungen des Zusammenhangs von Budgetdefiziten, Geldmenge und Inflation in den USA

Untersuchungen mit dem Ziel, die Geldmengenwirkungen staatlicher Budgetdefizite zu analysieren, sind eigentlich erst durch die These

1) Vgl. Roesch (1975), insbesondere S. $3 \mathrm{f}$.

2) Ebenda, S. 2. Vgl, auch Spindler (1979), S. 383

3) Vgl. auch Hein (1981), S. 7 ff. Er stellt fest, daß der vor 1975 erkennbare Zusammenhang zwischen Defiziten und Geldmenge in der Zeit zwischen 1975 und 1980 nicht mehr galt. 
von BW ausgelöst worden ${ }^{1)}$. Die Mehrzahl der vor 1977 veröffentlichten Arbeiten verfolgte primär das Ziel, Reaktionsfunktionen der Zentralbank auf Abweichungen wirtschaftspolitischer Ziele von ihren Wunschwerten zu testen ${ }^{2)}$, so daB sich der EinfluB fiskalischer Variablen auf die Geldmenge lediglich als Nebenprodukt ermitteln $1 a ̈ B t$. Die vorliegende Arbeit geht daher nur am Rande auf diese Studien ein und konzentriert sich auf die zur Oberprüfung der These von $B W$ erschienenen Beiträge. Zusätzlich wird die fast gleichzeitig mit der Schrift "Democracy in Deficit" erschienene Arbeit von SHARP/ FLENNIKEN ${ }^{3}$ ) diskutiert, die sowohl die Geldmengen-als auch die Preisniveauwirkungen staatlicher Budgetdefizite bestreitet.

Neben SHARP/FLENNIKEN hat sich vor allem BARRO ${ }^{4}$ ) gegen die These der Geldmengenwirkungen staatlicher Budgetdefizite gewandt. Seiner Ansicht nach wird die durch $D M_{t}=\log \left(M_{t}\right)-\log \left(M_{t-1}\right)$ repräsentierte Wachstumsrate der Geldmenge ${ }^{5}$ ) nicht durch den Budgetsaldo (SUR) ${ }^{6)}$, sondern durch die Wachstumsrate der realen Staatsausgaben der Zentralregierung in Relation zu einem "Normalniveau" (FEDV) ${ }^{7}$ ) beeinfluBt. BARRO testet diese Hypothese durch Schätzung der Koeffizienten der Gleichung

$$
D M_{t}=a+b \cdot D M_{t-1}+c \cdot D M_{t-2}+d \cdot U_{t-1}+e \cdot F E D V_{t}+f \cdot S U R_{t}+\varepsilon_{t}^{8)}
$$

1) Vgl. jedoch die noch zu diskutierende Studie von Sharp/Flenniken (1978).

2) Vgl. Z.B. Wood (1967), Friedlaender (1973) und Froyen (1974)

3) Vgl. Sharp/Flenniken (1978)

4) Vgl. Barro (1978) und (1978a)

5) $M$ ist ein Jahresdurchschnitt der Geldmenge $M_{1}$.

6) SUR wird in der von Barro zur Schätzung seiner These verwendeten Modeligleichung als Quotient des nominalen Budgetuberschusses der Zentralregierung und dem Produkt aus Preisindex des Bruttosozialprodukts und einem Trendwert des realen Bruttosozialprodukts gemessen. Vgl. Barro (1978), S. 577

7) Vgl. ebenda, S. $576 \mathrm{ff}$., aber auch Barro (1977). Für FEDV gilt FEDV $=\log \left(F_{E D}\right)-[\log (F E D)]_{t}^{\star}$, wobe $i$ FED die realen Staatsausgaben und $[\log (F E D)]_{t}^{*}$ eine Lag-Verteilung gegenwärtiger und vergangener Werte von $\log (F E D)$ repräsentieren, vgl. Barro (1978), S. 577.

8) $U_{t}$ ist ein MaB für die Arbeitslosigkeit und errechnet sich als log [ALQ/1-ALQ]. Vgl. Barro (1978), S. 577, $\varepsilon_{t}$ ist eine normalverteilte Störgröße. 
in drei Varianten.

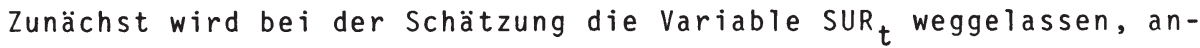
schließend die Variable FEDV $t$. In einer dritten Schätzung sind beide Variablen in der Gleichung enthalten. Für den Zeitraum von 1946 1976 stellt BARRO fest, daB die Variable FEDV $t$ in beiden Gleichungen, in denen sie enthalten ist, einen statistisch signifikanten Einfluß auf die Wachstumsrate der Geldmenge ausübt, die Variable SUR $_{t}$ jedoch nicht ${ }^{1}$ ). Für 1941 - 1976 ermittelt er analoge Ergeb-

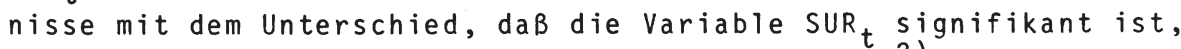
wenn FEDV $t_{t}$ nicht in die Gleichung einbezogen wird ${ }^{2}$ ).

Der Vergleich einer Wachstumsrate der Ausgaben mit einer Quote des Budgetsaldos erscheint indessen wenig aussagefähig. HAMBURGER/ ZWICK ${ }^{3)}$ ersetzen daher die Größe FEDV in BARROs Modellgleichung durch die Variable FE, die als Quote der Staatsausgaben und dem Produkt aus Preisindex des Bruttosozialprodukts und Trendwert des realen Bruttosozialprodukts definiert ist ${ }^{4}$.

Die für den Zeitraum von 1954 - 1976 ermittelten Schätzungen bestätigen die These BARROs, daB die Ausgaben und nicht die Defizite das Geldmengenwachstum bestimmen ${ }^{5)}$. Wird dieselbe Modellgleichung jedoch nach Eliminierung der statistisch nicht signifikanten Variablen $D M_{t-2}$ und $U_{t-1}$ für den Zeitraum 1961 - 1974, also die "keynesianische Periode" getestet, so ergeben sich die entgegengesetzten Konsequenzen. Der Koeffizient der Defizitquote ist in beiden Gleichun-

1) Barro ermittelt folgende Bestimmtheitsmaße: $R^{2}=0,60(0,50)$, wenn FEDV ${ }_{t}\left(\right.$ SUR $\left._{t}\right)$ allein in der Gleichung enthalten ist. Bei Berücksichtigung beider Variablen ist $R^{2}=0,71$.

2) $R^{2}=0,77(0,70)$ bei alleiniger Berücksichtigung von FEDV ${ }_{t}\left(\right.$ SUR $\left._{t}\right), R^{2}=0,80$ bei Berücksichtigung beider Variablen.

3) Vgl. Hamburger/Zwick (1981)

4) $\mathrm{Vgl}$. ebenda, S. $143 \mathrm{f}$.

5) Vgl. ebenda, S. 144 
gen, in denen er enthalten ist, statistisch signifikant von Null verschieden, der Koeffizient der Ausgabenquote nicht ${ }^{1}$.

Diese Ergebnisse legen den Schluß nahe, daß Defizite das Geldmengenwachstum in den USA zumindest in der "keynesianischen Periode" erhöht haben. Eine von FROYEN ${ }^{2}$ ) auf der Basis von Monatsdaten durchgeführte Untersuchung bestätigt die Ergebnisse von HAMBURGER/ ZWICK. FROYEN teilt den von ihm untersuchten Gesamtzeitraum von 1953 - 1972 in drei Unterzeiträume ein und testet für diese Unterzeiträume Reaktionsfunktionen der amerikanischen Zentralbank, die neben den Abweichungen wirtschaftspolitischer Ziele von ihren Wunschwerten auch das Budgetdefizit der Zentralregierung enthalten. Dabei konnte in den Zeiträumen ${ }^{3)}$ von 1961:2 - 1969:1 und 1969:2 1972:12 ein statistisch signifikanter EinfluB des Budgetdefizits auf die monetäre Basis festgestellt werden, im Zeitraum von 1953:2 1961:1 dagegen nicht ${ }^{4}$ ).

Der Unterschied in den Ergebnissen von HAMBURGER/ZWICK und BARRO scheint indessen weniger in der Berücksichtigung des Zeitraums vor 1961 als in der Berücksichtigung der Jahre 1975 und 1976 zu liegen. In diesen beiden Jahren der Ford-Administration erwirtschafteten die USA hohe Defizite, die Zentralbank widersetzte sich aber der Forderung, diese zu monetisieren. Schon 1977 und 1978 unter Präsident Carter wurde diese restriktive Haltung jedoch wieder aufgegeben ${ }^{5)}$. In der Tat dürfte die Einbeziehung dieser beiden Jahre eines atypischen Verhaltens der Zentralbank in den Schätzzeitraum die Ergebnisse BARROs entscheidend beeinflußt haben. Eine eigene Schät-

1) $R^{2}$ ist mit 0,66 am höchsten, wenn nur der Budgetsaldo in der Modellgleichung enthalten ist. Wird lediglich FE berücksichtigt, ist $R^{2}=0,58$, sind beide Variablen in der Gleichung vertreten, ist $R^{2}=0,64$.

2) Vgl. Froyen (1974)

3) Die hinter dem Doppelpunkt angegebene Zahl bezeichnet den jeweiligen Monat.

4) Vgl. Froyen (1974), Tabelle 1, S. 182

5) Vgl. auch Hamburger/Zwick (1981), S. $147 \mathrm{f}$. 
zung der Koeffizienten der Gleichung ${ }^{1)}$

$(v .16) \quad W_{M_{t}}=g+h \cdot W_{M_{t-1}}+i \cdot D_{t}+k \cdot U_{t-1}+\delta_{t}$

ermittelt für den Zeitraum von 1949 - 1974 einen statistisch signifikanten EinfluB der Defizitquote auf das Geldmengenwachstum. Die geschätzten Koeffizienten sind aus der nachfolgenden Gleichung ersichtlich.

$$
W_{M_{t}}=0,0941+\underset{(28,702)}{0,7295^{\star *}} \cdot W_{M_{t-1}}+\underset{(4,624)}{0,7764 *} \cdot D_{t}
$$

$(v .16 .1)$

$$
\begin{aligned}
& +\underset{(5,540)}{0,0300 *} \cdot U_{t-1} \\
& \left(R^{2}=0,602 ; F G=22\right)^{2)} .
\end{aligned}
$$

Diese Resultate erhärten die Behauptung, daß Defizite in der Vergangenheit zu einer Steigerung des Geldmengenwachstums in den USA geführt haben, und daB es sich bei den Jahren 1975 und 1976 um Ausnahmefälle handelte ${ }^{3)}$. Abschließend ist auf die bereits erwähnte Arbeit von SHARP/FLENNIKEN einzugehen, die die These der Geldmengen-

1) $W_{M}$ ist die Wachstumsrate der Geldmenge $M_{q}$, die auf der Grundlage von Jahresendwerten berechnet wurde. $D$ ist.der Quotient aus dem Budgetdefizit der Zentralregierung und dem Produkt aus dem Trendwert des realen Bruttosozialprodukts und dem Preisindex des Bruttosozialprodukts, $U_{t}$ entspricht dem Beschäftigungsmaß Barros. Vgl. zur Datenbasis Fußnote 1, S. 148.

2) Der zum Test auf Autokorrelation zu errechnende $h$-Wert wird im folgenden $n$ icht aufgeführt, da der $h$-Test in einigen Fällen versagt. Vgl. Johnston (1972), S. 313. Die Durbin-Watson-Koeffizienten, die bei derart kurzen Untersuchungsperioden allerdings in Richtung auf den Wert 2 verzerrt sind, lagen durchweg über 2.

3) Vgl. auch Hamburger/Zwick (1981), S. 148, die mittels einer Ex-post-Prognose zeigen, daß der von Buchanan/Wagner behauptete Zusammenhang 1977 und 1978 wieder Gültigkeit hatte. Im übrigen ermittelt Niskanen (1978) für den Gesamtzeitraum von 1948 - 1976 einen statistisch signifikanten EinfluB der Defizite auf die Geldmenge $M_{1}$. Se ine Untersuchung ist aber nicht ohne weiteres mit den übrigen vergleichbar, da er als abhängige Variable die Wachstumsrate der Geldmenge $M_{1}$, als unabhängige Variable den Quotienten aus Budgetdefizit und Geldmenge der Vorperiode verwendet. Vgl. ebenda, S. $598 \mathrm{ff}$. 
erhöhung durch staatliche Budgetdefizite bestreiten. Die Vorbehalte der beiden Autoren scheinen jedoch eher auf ihre Definition der defizitinduzierten Geldmengenerhöhung zurückzuführen zu sein als auf abweichende empirische Ergebnisse.

SHARP/FLENNIKEN versuchen, die durch die Schuldenerhöhung verursachte primäre Geldmengenerhöhung von der hierdurch ermöglichten multiplen Geldschöpfung zu isolieren: ". . only the initial increase in demand deposits can be directly attributable to budget deficits. . The multiple expansion in demand deposits that can occur when cash reserves are increased is attributable to the behavior of the private economy"). Die Geldmengenwirkung von Budgetdefiziten kann nach dieser Auffassung durch die Erhöhung des Bestands an staatlichen Wertpapieren im Portefeuille der Banken gemessen werden ${ }^{2}$.

Es ist indessen nicht einzusehen, warum durch Defizite bedingte Erhöhungen des Geldschöpfungsspielraums nicht in die Untersuchungen der Geldmengeneffekte von Defiziten einbezogen werden sollen. Wird durch Defizite eine zusätzliche multiple Geldschöpfung bewirkt, die ohne die staatliche Verschuldung nicht entstanden wäre, so dürfen diese Effekte nicht vernachlässigt werden. Dieser Einwand erscheint gravierend genug, die Untersuchung von SHARP/FLENNIKEN nicht als Falsifizierung der These von BW anzusehen ${ }^{3)}$.

Somit scheint es berechtigt, von der Giultigkeit des von BW postulierten Zusammenhangs zwischen Budgetdefiziten und der Geldmenge auszugehen. Damit ist jedoch die Frage nach dem AusmaB der durch Defizite bedingten Geldmengensteigerung noch nicht beantwortet. Während NISKANEN eine etwa 15 - 20 \% ige Monetisierung des Defizits in

1) Sharp/Flenniken (1978), S. 120

2) $\mathrm{Vgl}$. ebenda, S. 119

3) Der Einwand von Spindler (1979), S. 384, der als relevante Defizitvariable das Defizit analog zum "full employment budget" bei einem gegebenen Volkseinkommen betrachten will, erscheint dagegen nicht den Kern des Problems zu treffen, da der Defizit/Geldmengen-Zusammenhang für das tatsächliche und nicht für ein hypothetisches. Defizit behauptet wird. Vgl. Sharp (1979), S. 388 
der Zeit von 1948 - 1976 feststel1t ${ }^{1}$ ), gehen HAMBURGER/ZWICK von einer 20 - 25 \%igen Monetisierung während der "keynesianischen Periode" aus" 2 ). Beide Arbeiten weisen jedoch darauf hin, daB die Resultate aufgrund der Kürze der Untersuchungsperiode mit Vorsicht zu interpretieren seien. Speziell in der letztgenannten Untersuchung liegt jedoch auch bei der mathematischen Ermittlung des MonetisierungsausmaBes ein Fehler vor. HAMBURGER/ZWICK gehen mit Recht davon aus, daB der Koeffizient vor der Variablen SUR ${ }^{3)}$ in Gleichung (V.15) die Ableitung der Wachstumsrate der Geldmenge nach dem Quotienten aus Budgetsaldo und dem Produkt aus Sozialproduktstrendwert und Preisindex des Bruttosozialprodukts ist ${ }^{4)}$. Nichts einzuwenden ist auch gegen das Vorgehen, dieses Produkt durch das nominale Bruttosozialprodukt zu approximieren. Der Koeffizient repräsentiert dann den Ausdruck 5 )

$(v .17) \quad f=\frac{\partial\left(\frac{\Delta M}{M}\right)}{\partial\left(\frac{S}{B S P}\right)}$.

Durch Multiplikation dieses Ausdrucks mit dem zuvor als Durchschnitt der Jahre 1961 - 1974 errechneten Quotienten M/BSP läBt sich jedoch entgegen der Ansicht der beiden Autoren der Monetisierungsprozentsatz des Defizits $\Delta M / S$ mathematisch nicht ermitteln.

Der ermittelte Monetisierungsprozentsatz stellt daher allenfalls eine erste Approximation des tatsächlichen MonetisierungsausmaBes dar, die mit umso gröBeren Ungenauigkeiten behaftet ist, je gröBer

1) Vgl. Niskanen (1978), S. 601

2) Vgl. Hamburger/Zwick (1981), S. 146

3) Hamburger/Zwick verwenden im Zähler anstelle des Budgetüberschusses das Defizit. Im folgenden wird wegen der Bezugnahme auf Gleichung (V.15) weiterhin das Symbol SUR verwendet.

4) Vgl. Hamburger/Zwick (1981), S. 146

5) S repräsentiert den nominalen Budgetüberschuß, d.h. es gilt: $S=(S U R) \cdot\left(B S P_{\text {Trend }}\right)$. Im Falle eines Defizits hat $S$ ein negatives Vorzeichen. 
das durch die Schätzung ermittelte Absolutglied ist. Da HAMBURGER/ ZWICK in einer Schätzung ein Absolutglied von nur 0,01 ermittelten ${ }^{1)}$, dürfte der von ihnen angegebene Monetisierungsbereich von $20-25 \%$ in diesem speziellen Fall eine relativ gute Annäherung darstellen. Für das weitere Vorgehen wird jedoch, da mit einem Absolutglied von nahe Null a priori nicht gerechnet werden kann, ein alternativer Ansatz vorgeschlagen.

Die Verwendung des Quotienten aus Budgetsaldo und Sozialproduktstrendwert ist in den genannten Ansätzen wahrscheinlich durch das Bestreben zur Vermeidung von Multikollinearität zu erklären. Multikollinearität läBt sich jedoch auch durch die Verwendung von Differenzen vermeiden, eine Vorgehensweise, die angesichts der obigen Kritikpunkte überlegen erscheint. Die Verwendung von Differenzen bietet den zusätzlichen Vorteil, daB auch der EinfluB zyklischer Anderungen der wirtschaftlichen Aktivität auf Geldmengenvariationen, der in den Ansätzen von BARRO und HAMBURGER/ZWICK aufgrund der Verwendung eines Sozialproduktstrendwerts nicht erfaBt wird, ermittelt werden $k a n n^{2}$ ).

Im folgenden werden daher Modellgleichungen mit der Anderung der Geldmenge $M_{1}(\Delta M)$ als abhängiger Variabler getestet. Als unabhängige Variable werden neben der Geldmengenänderung der Vorperioden Budgetdefizite (DEF) der Zentralregierung, die Anderung des realen Bruttosozialprodukts $\left(\Delta Y^{r}\right)$ und das von BARRO verwendete BeschäftigungsmaB $U_{t-1}$ verwendet. Tabelle 13 (siehe $S .157$ ) gibt einen Oberblick über die Schätzungen.

Sowohl bei Verwendung von Ursprungswerten als auch bei Verwendung von trendbereinigten Werten ist der Koeffizient der Defizitvariablen statistisch signifikant von Null verschieden, wenn die nicht

1) Vgl. Hamburger/Zwick (1981), S. 144

2) Wïrde man anstelle des Sozialproduktstrendwerts das tatsächliche BSP als Divisor verwenden, so ließe sich der EinfluB des Defizits nicht mehr von dem des Sozialprodukts trennen. 


\begin{tabular}{|c|c|c|c|c|c|c|c|c|}
\hline $\begin{array}{l}\text { Gleichungs- } \\
\text { nummer }\end{array}$ & $\Delta M_{t-1}$ & $\Delta M_{t-2}$ & DEF & $\Delta r^{r}$ & $u_{t-1}$ & $\begin{array}{l}\text { Absolut- } \\
\text { gl ied }\end{array}$ & $\begin{array}{l}\text { Testst } \\
R^{2}\end{array}$ & $\begin{array}{l}\text { istiken } \\
\text { FG }\end{array}$ \\
\hline (V.18.1.1) & $\begin{array}{l}0,5525 * \star \\
(14,763)\end{array}$ & $\begin{array}{l}0,3376 \\
(3,133)\end{array}$ & $\begin{array}{l}0,1282 \\
(3,223)\end{array}$ & $\begin{array}{l}0,0855^{\star \star \star} \\
(13,892)\end{array}$ & $\begin{array}{l}0,6570 \\
(0,131)\end{array}$ & 0,0834 & 0,897 & 20 \\
\hline (V.18.2.1) & $\begin{array}{l}0,7652 \star \star \\
(85,175)\end{array}$ & - & $\begin{array}{l}0,1962 * \star \\
(9,652)\end{array}$ & $\begin{array}{l}0,0690 * \star \\
(9,837)\end{array}$ & $\begin{array}{l}0,3091 \\
(0,027)\end{array}$ & $-0,3945$ & 0,881 & 21 \\
\hline (V.18.3.1) & $\begin{array}{l}0,5531 * \star \\
(15,436)\end{array}$ & $\begin{array}{l}0,3301 \\
(3,163)\end{array}$ & $\begin{array}{l}0,1295 \\
(3,438)\end{array}$ & $\begin{array}{l}0,0892 \star \star \\
(19,810)\end{array}$ & - & $-1,9790$ & 0,897 & 21 \\
\hline (V.18.4.1) & $\begin{array}{l}0,7633 * \star \\
(90,544)\end{array}$ & - & $\begin{array}{l}0,1961 \star \star \\
(10,089)\end{array}$ & $\begin{array}{l}0,0710 \star \star \\
(15,473)\end{array}$ & - & $-1,3712$ & 0,881 & 22 \\
\hline $\begin{array}{l}\text { (V.18.1.2) } \\
\text { (trendbereinigt) }\end{array}$ & $\begin{array}{l}0,5153 * * \\
(9,031)\end{array}$ & $\begin{array}{l}0,3149 \\
(2,430)\end{array}$ & $\begin{array}{l}0,1196 \\
(2,491)\end{array}$ & $\begin{array}{l}0,0847 * \star \\
(13,020)\end{array}$ & $\begin{array}{l}0,2928 \\
(0,020)\end{array}$ & $-1,3164$ & 0,898 & 19 \\
\hline $\begin{array}{l}\text { (V.18.2.2) } \\
\text { (trendbereinigt) }\end{array}$ & $\begin{array}{l}0,6629 * \star \\
(20,074)\end{array}$ & - & $\begin{array}{l}0,1700^{\star} \\
(5,754)\end{array}$ & $\begin{array}{l}0,0697 \star \star \\
(9,891)\end{array}$ & $\begin{array}{l}-0,3679 \\
(0,032)\end{array}$ & $-3,1115$ & 0,885 & 20 \\
\hline $\begin{array}{l}\text { (v.18.3.2) } \\
\text { (trendbereinigt) }\end{array}$ & $\begin{array}{l}0,5101 * * \\
(9,734)\end{array}$ & $\begin{array}{l}0,3089 \\
(2,569)\end{array}$ & $\begin{array}{l}0,1188 \\
(2,599)\end{array}$ & $\begin{array}{l}0,0860 * * \\
(16,422)\end{array}$ & - & $-2,2726$ & 0,898 & 20 \\
\hline $\begin{array}{l}\text { (V.18.4.2) } \\
\text { (trendbereinigt) }\end{array}$ & $\begin{array}{l}0,6733 * \star \\
(25,766)\end{array}$ & - & $\begin{array}{l}0,1723^{*} \\
(6,403)\end{array}$ & $\begin{array}{l}0,0677 \star \star \\
(13,332)\end{array}$ & - & $-1,9010$ & 0,885 & 21 \\
\hline
\end{tabular}

Tabelle 13: Geldmengenwirkungen von Budgetsalden in den USA im Zeitraum von 1949-19741)

1) Datenquelle: Vgl. Tabelle 12, S. 148, ** kennzeichnet ein Signifikanzniveau von $99 \%$, ein Signifikanzniveau von $95 \%$. Die Trendbereinigung wurde durch Aufnahme einer Trendvariablen in die Schätzgleichung vorgenommen, so daB $R^{2}$ auch den Trende influB beinhal tet. 
signifikante Variable $\Delta M_{t-2}$ nicht in der Gleichung enthalten ist ${ }^{1}$. Die Ergebnisse legen den SchluB nahe, daB im Zeitraum von 1949 1974 die Anderung des Budgetsaldos um 1 Mrd. zu einer Anderung von $\triangle M$ um etwa 0,2 Mrd. führte. Die Ergebnisse von NISKANEN und HAMBURGER/ZWICK werden dadurch in der Tendenz bestätigt. Weiterhin ist ersichtlich, daB die Aufnahme einer Trendvariablen den Wert der Koeffizienten und das Bestimmtheitsmaß nur geringfügig ändert.

Allerdings $1 \ddot{B} B t$ sich auch für $\Delta M_{t-2}$ ein statistisch signifikant von Null verschiedener EinfluB ermitteln, wenn die Variable DEF aus der Gleichung eliminiert wird. Da das Bestimmtheitsma $B$ in diesem Fal1 nur geringfügig niedriger ist, kann nicht eindeutig geklärt werden, ob $D E F$ oder $\Delta M_{t-2}$ das aktuelle Geldmengenwachstum besser erk1ären ${ }^{2)}$.

Wird allerdings unterstellt, daB Budgetsalden nur im Falle von Budgetdefiziten einen EinfluB auf Geldmengenänderungen ausüben - eine Hypothese, die sich durch Berücksichtigung einer Dummy-Variablen überprüfen läBt -, so ist der Koeffizient von DEF signifikant, derjenige von $\Delta M_{t-2}$ nicht. Die für die Variable DEF geschätzten Koeffizienten sind höher, was auf eine stärkere Monetisierung im Defizitfall schließen 1äßt. Die Schätzergebnisse sind in Tabelle 14 (siehe S. 159) zusammengefaßt.

Angesichts der Bedeutung des Ziels der Preisniveaustabilität in der Bundesrepublik Deutschland wurden zur Erleichterung des Vergleichs noch einige Modellgleichungen getestet, in denen die Variable $U_{t-1}$ durch die Preissteigerungsrate $W p_{t} b z w$. Wp $p_{t-1}$ ersetzt wurde. MiBt das FRS dem Ziel der Preisniveaustabilität Bedeutung zu, so müßte für diese Variablen ein negativer Koeffizient zu ermitteln sein.

1) Da das BestimmtheitsmaB im Falle von (V.18.2) jeweils nur geringfügig höher ist als im Falle von (V.18.4) - das auf 3 Stellen gerundete BestimmtheitsmaB ist identisch -, wird im folgenden anhand der Gleichung (V.18.4) argumentiert.

2) Auf die Wiedergabe der geschätzten Koeffizienten wird an dieser Stelle verzichtet. 


\begin{tabular}{|c|c|c|c|c|c|c|c|c|}
\hline $\begin{array}{l}\text { Gleichungs- } \\
\text { nummer }\end{array}$ & $\Delta M_{t-1}$ & $\Delta M_{t-2}$ & DEF & $\Delta Y^{r}$ & $U_{t-1}$ & $\begin{array}{l}\text { Absolut- } \\
\text { glied }\end{array}$ & $\begin{array}{l}\text { Testst } \\
\mathrm{R}^{2}\end{array}$ & $\begin{array}{l}\text { istiken } \\
\text { FG }\end{array}$ \\
\hline (v.18.1.3) & $\begin{array}{l}0,5322^{\star \star} \\
(15,684)\end{array}$ & $\begin{array}{l}0,3218 \\
\quad(3,259)\end{array}$ & $\begin{array}{l}0,1785^{\star} \\
\quad(5,086)\end{array}$ & $\begin{array}{l}0,0827 \star \star \\
(14,070)\end{array}$ & $\begin{array}{l}0,8229 \\
\quad(0,222)\end{array}$ & 0,4401 & 0,905 & 20 \\
\hline (V.18.2.3) & $\begin{array}{l}0,7283^{\star \star} \\
(76,432)\end{array}$ & - & $\begin{array}{l}0,2496 \star \star \\
(11,933)\end{array}$ & $\begin{array}{r}0,0668^{\star \star} \\
(9,864)\end{array}$ & $\begin{array}{l}0,5599 \\
\quad(0,094)\end{array}$ & 0,1565 & 0,889 & 21 \\
\hline (V.18.3.3) & $\begin{array}{l}0,5320 \text { ** } \\
(16,275)\end{array}$ & $\begin{array}{l}0,3148 \\
\quad(3,261)\end{array}$ & $\begin{array}{l}0,1785^{\star} \\
\quad(5,279)\end{array}$ & $\begin{array}{l}0,0876 \star \star \\
(20,993)\end{array}$ & - & $-2,1444$ & 0,904 & 21 \\
\hline (V.18.4.3) & $\begin{array}{l}0,7252^{\star \star} \\
\quad(80,215)\end{array}$ & - & $\begin{array}{l}0,2485^{\star \star} \\
\quad(12,367)\end{array}$ & $\begin{array}{l}0,0704 * \star \\
\quad(16,347)\end{array}$ & - & $-1,6099$ & 0,889 & 20 \\
\hline
\end{tabular}

Tabelle 14: Geldmengenwirkungen von Budgetdefiziten in den USA von 1949-19741)

1) Vgl. auch die Hinweise zu Tabelle 13 
Der Koeffizient der laufenden Preissteigerungsrate erwies sich zwar als statistisch signifikant auf dem $95 \%-N i v e a u$. Sein positives Vorzeichen $1 a ̈ \beta t$ jedoch eher darauf schließen, daß die Kausalkette in umgekehrter Richtung verläuft, daB also nicht die Zentralbank auf Preissteigerungen reagiert, sondern daB Geldmengenänderungen die Inflationsrate erhöhen ${ }^{1)}$. Diese These 1 äBt sich in der Tat empirisch belegen. Die geschätzten Koeffizienten der Gleichung (V.19) deuten auf einen EinfluB der Geldmengenänderung der Vorperiode auf die laufende Preissteigerungsrate hin.

( V.19)

$$
W_{p_{t}}=0,0118+\frac{0,003126 * \star}{(30,256)} \cdot \Delta M_{t-1}
$$

$$
\left.\left(R^{2}=0,558 ; D W=1,8148 ; F G=24\right)^{2}\right) \text {. }
$$

Eine Beeinflussung der Preissteigerungsrate durch statiche Budgetdefizite ist damit jedoch noch nicht nachgewiesen, obwohl aufgrund der Geldmengenwirkung von Budgetdefiziten oft auf eine durch sie bedingte Inflationserhöhung geschlossen wird ${ }^{3)}$. Empirische Untersuchungen ${ }^{4}$ legen indessen eher die gegenteilige These nahe.

Zur Untersuchung der Frage, ob Budgetdefizite die Inflationsrate erhöhen, wird im folgenden die monetaristische These zugrundegelegt, daB Geldmengenerhöhungen dann inflatorisch wirken, wenn die Wachstumsrate der Geldmenge $W_{M}$ die Wachstumsrate des realen Bruttosozialprodukts $W_{Y r}$ übersteigt ${ }^{5}$. LäBt sich diese These empirisch bestätigen und kann weiterhin ein bedeutender EinfluB von Budgetdefiziten auf die Differenz $\left(W_{M}-W_{Y_{r}}\right)$ nachgewiesen werden, so könnte man von einer inflationären Wirkung der Staatsverschuldung

1) Für diese These spricht auch die Tatsache, daB für $W_{p_{t-1}}$ ke in statistisch signifikanter EinfluB auf $\Delta M_{t}$ festgestellt werden konnte.

2) DW kennzeichnet den Durbin-Watson-Koeffizienten.

3) So auch Hamburger/Zwick (1981), S. 149. Vgl. aber Hein (1981), S. 4

4) Vg1. Z.B. Niskanen (1978), S. 601 sowie Sharp/Flenniken (1978), S. 123 ff.

5) Vgl. Z.B. Neumann (1981), S. 122 
ausgehen. In der Tat läBt sich in der Zeit von 1949 - 1974 ein statistisch signifikanter EinfluB der Differenz ( $W_{M}-W_{Y r}$ ) auf die Inflationsrate $W_{p}$ nachweisen:

( V.20)

$$
w_{p_{t}}=0,0302+\underset{(10,254)}{0,3964 * *} \cdot\left(w_{M}-w_{Y_{r}}\right)_{t}
$$

$$
\left(R^{2}=0,299 ; D W=1,6574 ; F G=24\right) \text {. }
$$

Weiterhin kann ein EinfluB von Budgetdefiziten auf die Differenz der genannten Wachstumsraten ermittelt werden:

$$
\left(W_{M}-W_{Y_{r}}\right)_{t}=-0,0123+\underset{(4,824)}{0,00179 *} \cdot D E F_{t}
$$

$$
\left(R^{2}=0,167 ; D W=1,3132 ; F G=24\right) \text {. }
$$

Das BestimmtheitsmaB ist aber in beiden Fällen so niedrig, daB Zweifel an der Gültigkeit des behaupteten Zusammenhangs angebracht erscheinen.

Ein statistisch signifikanter EinfluB von Budgetsalden auf die Preissteigerungsrate läBt sich jedenfalls in der betrachteten Zeitperiode nicht ermitteln ${ }^{1}$ ).

Somit bleibt festzuhalten, daB Budgetdefizite in den USA im Zeitraum von 1949 - 1974 zu Geldmengenänderungen geführt haben, daB das AusmaB der Geldmengenwirkung jedoch nicht so groB war, daB von Budgetdefiziten wesentliche Inflationswirkungen ausgegangen sind. Die These von BW, Budgetdefizite hätten seit Beginn der Sechziger Jahre maBgeblich zu den hohen Inflationsraten in den Vereinigten Staaten beigetragen, ist daher zu relativieren ${ }^{2}$.

Nachdem ein Geldmengeneffekt staatlicher Budgetdefizite empirisch bestätigt werden konnte, interessiert angesichts der Tatsache, daß

1) Auf die Wiedergabe der Schätzergebnisse kann daher verzichtet werden.

2) Vgl. auch Hein (1981), S. 7, der den Defizit/Geldmengen-Zusammenhang in der Zeit zwischen 1975 und 1980 bestreitet. 
die Geldmenge $M_{1}$ nicht nur durch MaBnahmen der Zentralbank, sondern auch durch Publikumsimpulse beeinfluBt wird, naturlich die Frage, inwieweit die festgestellte Monetisierung der Statsschuld auf Maßnahmen des FRS zurückzuführen ist.

Den Versuch, den Zentralbankeinfluß vom Publikumseinfluß zu isolieren, haben MCMILLIN/BEARD ${ }^{1)}$ unternommen. In ihrem ökonometrischen Mehrgleichungsmodel1, einer 1 inearen Variante des IS/LM-Modells unter Berücksichtigung von Vermögenseffekten, unterscheiden die Autoren zwei Fälle: Im ersten Fall wird die Zentralbank als exogen betrachtet, so daB fiskalpolitisch induzierte Geldmengenänderungen durch Anpassungsreaktionen im privaten Sektor bedingt sein müssen. Im zweiten Fall wird das Verhalten der Zentralbank durch Berücksichtigung einer Reaktionsfunktion ${ }^{2)}$ endogenisiert, so daB der EinfluB der Fiskalpolitik auf die Geldpolitik sowohl auf Reaktionen der Privaten als auch auf Reaktionen der Zentralbank zurückzuführen ist. Ein Vergleich der in beiden Fällen ermittelten impact multipliers läßt dann Rückschlüsse darauf zu, wie die Zentralbank kurzfristig (d.h. innerhalb desselben Quartals) auf fiskalpolitische Maßnahmen reagiert.

Im Falle einer exogenen Zentralbank wurde für marginale Staatsausgabenerhöhungen ein AnstoBmultiplikator von 0,054 festgestellt, d.h. die Erhöhung der Staatsausgaben um 1 Mrd. US- $\$$ führt c.p. noch in demselben Quartal zu einer Erhöhung der Geldmenge um 54 Mio. US $-\$$. Steuersenkungen um 1 Mrd. US-\$ erhöhen dagegen die Geldmenge c.p. um 11 Mio. US-\$.

Eine Endogenisierung des Zentralbankverhaltens führt zu einer beträchtlichen Erhöhung der Multiplikatoren. Der AnstoBmultiplikator

1) Vgl. McMillin/Beard (1980). Ihre auf der Basis von Quartalsdaten durchgeführte Untersuchung bezieht sich auf den Zeitraum von 1953 I - 1976 IV.

2) Der Ableitung der Reaktionsfunktion liegt wie im Ansatz von Basler die Minimierung einer quadratischen Nutzenverlustfunktion unter der Nebenbedingung der Wirtschaftsstruktur zugrunde. Vgl. zum formalen Ansatz McMillin/Beard $(1980)$, S. $133 \mathrm{ff}$. 
weist im Falle einer marginalen Ausgabenerhöhung einen Wert von 0,173 , im Falle einer marginalen Steuersenkung einen Wert von 0,165 auf ${ }^{1)}$. Diese Ergebnisse führen zu dem SchluB, daB das FRS in weitaus stärkerem Maße zur Monetisierung marginaler Budgetdefizite beigetragen hat als der private Sektor.

Der Grund für diese Tendenz könnte darin liegen, daß die Zentralbank auf die Stabilisierung des Zinsniveaus insgesamt gesehen stärkeren Wert legte als auf makroökonomische Ziele wie Preisniveaustabilität und Wirtschaftswachstum. Die von MCMILLIN/BEARD geschätzten Koeffizienten in der Reaktionsfunktion der Zentralbank deuten jedenfalls auf diese Zielgewichtung hin ${ }^{2}$.

In bezug auf die weitergehende Folgerung, eine expansive Fiskalpolitik würde - wie von BW behauptet - zu einer erheblichen Erhöhung der Geldmenge und damit zur Schaffung eines Inflationspotentials führen, mahnen die Autoren selbst jedoch ausdrücklich zur Vorsicht. Zunächst einmal kann man geteilter Meinung darüber sein, ob die ermittelten Multiplikatoren auf eine "beträchtliche" Monetisierung marginaler Budgetsalden hindeuten. AuBerdem lassen die AnstoBmultiplikatoren lediglich einen SchluB auf kurzfristige Geldmengenwirkungen der Fiskalpolitik zu. Die Frage, ob diese Auswirkungen auch in der Folgezeit zu einer dauerhaften Erhöhung der Geldmenge führen, kann ohne die Bestimmung dynamischer Multiplikatoren nicht definitiv entschieden werden ${ }^{3)}$. Die These von $B W$, das Wachstum der Staatsschuld in den USA habe zu einer beträchtlichen Erhöhung der Geldmenge und damit zur Schaffung eines Inflationspotentials geführt, kann daher empirisch nicht als bestätigt gelten.

1) Vgl. McMill in/Beard (1980), S. 132

2) Die Vorrangstellung des genannten Ziels gilt für die Zeit nach 1970 III allerdings nicht mehr uneingeschränkt. Vgl. ebenda, S. $131 \mathrm{ff} . \mathrm{Vgl}$. aber Pierce (1978), S. 366 und We intraub (1978), S. 358

3) Vgl. McMill in/Beard (1980), S. 132. Vgl. zu einem ersten Ansatz der Bestimmung dynamischer Multiplikatoren McMillin (1981), S. $224 \mathrm{f}$. 
2.2.2.2.2.3. Zusammenfassung der Ergebnisse für den Bereich der $\underline{U S A}$

Die Ergebnisse des vorigen Abschnitts lassen den SchluB zu, daB staatliche Budgetdefizite das Geldmengenwachstum in den USA bis zum Jahr 1974 beschleunigt haben, daß aber auch für diesen Zeitraum der weitergehende SchluB auf eine defizitbedingte Erhöhung der Inflationsrate nicht gezogen werden kann. Jedenfalls ist die Behauptung von BW, die wachsenden Budgetdefizite der Sechziger Jahre seien als eine Hauptursache der Inflation anzusehen, empirisch nicht belegbar. Weiterhin legt die Analyse von MCMILLIN/ BEARD den SchluB nahe, daB das FRS aufgrund einer faktisch schon immer bestehenden (und seit 1977 gesetzlich fixierten) 1 ) Verpflichtung zur Stabilisierung des Zinsniveaus entscheidend zur Monetisierung der Staatsschuld beigetragen hat.

\subsection{Geldmengen- und Inflationswirkungen von Budgetdefiziten} in der Bundesrepublik Deutschland

Bei der empirischen Untersuchung eines Zusammenhangs, der aufgrund theoretischer Erörterungen nicht begründbar ist, steht der Analytiker vor einer undankbaren Aufgabe, kann gegen die empirische Bestätigung seiner Aussage doch eingewandt werden, da $B$ ein alternatives empirisches Modell möglicherweise zur Falsifizierung der Aussage geführt hätte. Dieser Schwierigkeit sieht sich auch die vorliegende Arbeit gegenüber, da die Erörterungen über die Autonomie der Bundesbank zu der These führen müssen, daB Budgetdefizite das Geldmengenwachstum in der Bundesrepublik bisher nicht oder nur in sehr geringem Umfang tangiert haben.

Um der empirischen Analyse trotz dieser Schwierigkeiten Aussagekraft zu geben, werden im folgenden exakt dieselben Modellgleichungen

1) Gerade im Zeitraum nach 1977 ist der Zusammenhang zwischen Defiziten und Geldmenge jedoch nicht unbestritten. Vgl. Hein (1981), S. $6 \mathrm{ff}$. 
(

$$
M_{t}=a+b \cdot \Delta M_{t-1}+c \cdot \Delta M_{t-2}+d \cdot D E F_{t}+e \cdot \Delta Y_{t}^{r}+f \cdot U_{t-1}+\beta_{t}
$$

zugrundegelegt, anhand derer für die USA ein Einfluß von Defiziten auf Geldmengenänderungen festgestellt wurde.

Die Gleichungen (V.18.1.5) - (V.18.4.5) in Tabelle 15 geben die Schätzergebnisse für den Zeitraum von 1966 - 1979 wieder 1$)$. In einer der vier Gleichungen ist ein statistisch signifikanter Einfluß der Haushaltsdefizite auf die Anderung der Geldmenge $M_{1}$ festzustellen. Dieser Einfluß verschwindet jedoch, wenn man die stark trendbehafteten Variablen trendbereinigt (vgl. die Gleichungen (V.18.1.6) - (v.18.4.6)).

Es wird deutlich, daß lediglich die Geldmengenänderungen der Vorperioden einen statistisch signifikanten (negativen) Einfluß auf die Geldmengenänderung der laufenden Periode ausüben. Dieses Ergebnis führt zu dem Schluß, daß das Geldmengenwachstum in der Bundesrepublik Deutschland von der ökonomischen Entwicklung nicht systematisch beeinflußt wurde.

Zur Oberprüfung der Testergebnisse wurde das Modell geringfügig modifiziert. Für das von BARRO verwendete Beschäftigungsmaß wurde die Preissteigerungsrate ${ }^{2)}$ der laufenden bzw. der Vorperiode in die Schätzgleichung aufgenommen, ohne daß sich an den grundsätzlichen Ergebnissen etwas änderte.

Die Testergebnisse sind in Anbetracht der Kürze des Untersuchungszeitraums natürlich nur mit Vorsicht zu interpretieren. Auch die Tatsache, daB der Untersuchung lediglich ein Eingleichungsmodel 1 zugrundeliegt, muß zur Vorsicht mahnen. Mehr Klarheit könnte even-

1) Der Zeitraum ab 1966 wurde gewählt, weil vor dieser Zeit noch keine systematische keynesianische Fiskalpolitik betrieben wurde.

2) Die Preissteigerungsrate wurde anhand des Preisindex des Bruttosozialprodukts berechnet. Datenquelle: Verschiedene Ausgaben der Gutachten des Sachverständigenrats. 


\begin{tabular}{|c|c|c|c|c|c|c|c|c|}
\hline $\begin{array}{l}\text { Gleichungs- } \\
\text { nummer }\end{array}$ & $\Delta M_{t-1}$ & $\Delta M_{t-2}$ & $\mathrm{DEF}_{t}$ & $\Delta Y_{t}^{r}$ & $U_{t-1}$ & $\begin{array}{l}\text { Absolut- } \\
\text { glied }\end{array}$ & $\begin{array}{l}\text { Test- } \\
\text { statis }\end{array}$ & tiken \\
\hline & & & & & & & & $F G$ \\
\hline$(v .18 \cdot 1 . / 2.5)^{1)}$ & $\begin{array}{r}-0,7711 \\
(1,830)\end{array}$ & $\begin{array}{l}0,00 \\
(0,001)\end{array}$ & $\begin{array}{l}1,3717 \\
(2,678)\end{array}$ & $\begin{array}{l}0,3656 \\
(0,976)\end{array}$ & $\begin{array}{r}-17,6874 \\
(0,790)\end{array}$ & $-35,3524$ & 0,53 & 9 \\
\hline (V.18.3.5) & $\begin{array}{r}-0,4324 \\
\quad(0,980)\end{array}$ & $\begin{array}{l}-0,1062 \\
\quad(0,109)\end{array}$ & $\begin{array}{l}0,7041 \\
\quad(4,718)\end{array}$ & $\begin{array}{l}0,0879 \\
(0,231)\end{array}$ & - & 7,6708 & 0,50 & 9 \\
\hline (V.18.4.5) & $\begin{array}{r}-0,4297 \\
(1,063)\end{array}$ & - & $\begin{array}{l}0,6765^{\star} \\
(5,122)\end{array}$ & $\begin{array}{l}0,0733 \\
(0,190)\end{array}$ & - & 7,2327 & 0,49 & 10 \\
\hline $\begin{array}{l}(v .18 .1 .6)^{2)} \\
\text { (trendbereinigt) }\end{array}$ & $\begin{array}{r}-0,7329 \\
(4,443)\end{array}$ & $\begin{array}{l}-0,9984^{*} \\
(10,968)\end{array}$ & $\begin{array}{l}-0,0914 \\
(0,022)\end{array}$ & $\begin{array}{l}-0,0535 \\
(0,048)\end{array}$ & $\begin{array}{r}-2,5303 \\
(0,038) \\
\end{array}$ & $-10,7867$ & 0,87 & 7 \\
\hline $\begin{array}{l}(V .18 .2 .6) \\
\text { (trendbereinigt) }\end{array}$ & $\begin{array}{r}-0,6874^{\star} \\
(8,154)\end{array}$ & $\begin{array}{r}-1,0227 \star \star \\
(15,823)\end{array}$ & $\begin{array}{r}-0,1980 \\
(0,598)\end{array}$ & $\begin{array}{l}-0,0957 \\
(0,839)\end{array}$ & - & $-4,7859$ & 0,87 & 8 \\
\hline $\begin{array}{l}(V .18 .3 .6) \\
\text { (trendbereinigt) }\end{array}$ & $\begin{array}{r}-0,9206 \\
(3,207)\end{array}$ & - & $\begin{array}{l}1,0153 \\
(1,732)\end{array}$ & $\begin{array}{l}0,2890 \\
(0,757)\end{array}$ & $\begin{array}{r}-20,5112 \\
(1,331)\end{array}$ & $-48,8076$ & 0,67 & 8 \\
\hline (v.18.4.6) & $\begin{array}{r}-0,5195 \\
(1,815)\end{array}$ & - & $\begin{array}{l}0,2346 \\
\quad(0,387)\end{array}$ & $\begin{array}{r}-0,4315 \\
(0,066)\end{array}$ & - & 0,9399 & 0,62 & 9 \\
\hline
\end{tabular}

Tabelle 15: Geldmengenwirkungen von Budgetdefiziten in der Bundesrepublik Deutschland ${ }^{3}$ )

1) Eine Unterscheidung der Gleichungen (V.18.1) und (V.18.2) erübrigt sich aufgrund des zu vernachlässigenden Einflusses von $\Delta M_{t-2^{*}}$

2) Die Trendbereinigung wurde durch Einbeziehung einer Trendvariablen vorgenommen. Die Testgröße $R^{2}$ enthält demnach auch den Trendeinfluß.

3) Datenquelle: Monatsberichte der Deutschen Bundesbank (verschiedene Ausgaben). Die Sozialproduktswerte wurden auf der Basis der vom DIW herausgegebenen Langen Reihen zur VGR errechnet. Vgl. Deutsches Institut für Wirtschaftsforschung (1980) 
tuell ein ökonometrisches GroBmodell schaffen, dessen Konstruktion allerdings den Rahmen dieser Arbeit sprengen würde ${ }^{1}$ ).

Somit muB es bei der Feststellung bleiben, daB der für den Bereich der USA auf der Basis der Gleichung (V.18) ermittelte Zusammenhang zwischen Budgetdefiziten und Geldmengenänderungen in der Bundesrepublik im betrachteten Zeitraum nicht nachgewiesen werden kann. Die eingangs formulierte Hypothese, mit den in den USA festste11baren Geldmengenwirkungen von Budgetdefiziten sei in der Bundesrepublik nicht zu rechnen, wird somit durch die Empirie zumindest nicht widerlegt ${ }^{2}$ ). Akzeptiert man die monetaristische Behauptung, inflationäre Impulse würden lediglich durch eine die Wachstumsrate des realen Sozialprodukts übersteigende Wachstumsrate der Geldmenge ausgelöst, so folgt aus der fehlenden Verbindung zwischen Defiziten und Geldmengenänderungen auch, daß Budgetdefizite bisher noch keine inflatorischen Effekte ausgelöst haben ${ }^{3}$ ) ).

1) Zusätzlich wurden für den Zeitraum von 1973 bis Anfang 1980, also in der Zeit, in der die Höhe der jährlichen Budgetdefizite beträchtlich zunahm, noch Schätzungen auf der Basis von Quartalsdaten durchgeführt. Dabe i wurden wie im Rahmen der Analyse der Bundesbankautonomie "Reaktionsfunktionen" der Bundesbank getestet, denen spezielle Hypothesen über das Bundesbankverhalten zugrundeliegen. Bei Verwendung von Anderungen der Geldmenge $M_{1}$ als abhängiger Variabler konnte ein statistisch signifikanter EinfluB der Sozialproduktsänderung und der um zwei Perioden verzögerten Geldmengenänderung nachgewiesen werden. $\mathrm{Vgl}$. zu den Schätzergebnissen Anhang, Punkt 3 . Die Geldmenge $M_{1}$ ist indessen keine erklärte Zielvariable der Deutschen Bundesbank, so da $B$ von einer Reaktionsfunktion eigentlich gar nicht gesprochen werden kann. Bei Verwendung der erklärten Zielgröße, der saisonbereinigten Zentralbankgeldmenge zu konstanten Reservesätzen, war der Koeffizient der Sozialproduktsänderung indessen nicht mehr statistisch signifikant. Außerdem war das Bestimmtheitsmaß zu gering, als daß der Interpretation der Schätzergebnisse ein allzu großer Aussagegehalt zukommen würde.

2) Vgl. auch Nölling (1979), S. 483

3) Vgl. ebenda und Willms (1978), S. 443

4) Auch empirisch läßt sich auf der Basis von OLS-Schätzungen kein positiver Einfluß der Verschuldung auf die Preissteigerungsrate ermitteln. 
2.3. Fazit: Budgetdefizite, Geldmenge und Preisniveau in den USA und in der Bundesrepublik Deutschland

Sowohl die Ergebnisse der institutionellen Analyse als auch die empirischen Untersuchungen der von $B W$ behaupteten Zusammenhänge legen den Schluß nahe, daB Budgetdefizite das Geldmengenwachstum in den USA erhöht haben, in der Bundesrepublik dagegen nicht. Eine Inflationswirkung von Budgetdefiziten konnte empirisch weder für den Bereich der USA, noch für die Bundesrepublik Deutschland festgestellt werden.

Wie läßt sich dieses Ergebnis begründen? Die Ausführungen zu Beginn dieses Kapitels ${ }^{1}$ ) machen deutlich, daß mit einer Geldmengen- und damit mit einer Inflationswirkung statlicher Budgetdefizite nur dann zu rechnen ist, wenn die Zentralbank an einer Stabilisierung des Zinsniveaus interessiert ist bzw. zu dieser Maßnahme gezwungen werden $k a n n^{2}$ ).

Die divergierenden Ergebnisse im Hinblick auf die Geldmengeneffekte von Budgetdefiziten in den USA und in der Bundesrepublik durften daher vor allem durch die unterschiedliche Bedeutung des Zinsniveaus für Zentralbankentscheidungen in diesen staaten bedingt sein. Während das FRS im Untersuchungszeitraum ${ }^{3)}$ de facto eine Politik der Zinsstabilisierung verfolgt hat, ist eine derartige Politik der Deutschen Bundesbank empirisch nicht belegbar ${ }^{4}$.

1) Vgl. vor allem die Abschnitte V.1. und V.2.

2) Vgl. Neumann (1981), S. $124 \mathrm{ff}$. und He in (1981), S. 4

3) Es muß darauf hingewiesen werden, daß die These der Geldmengenwirkung von Budgetdefiziten nur bis zum Jahre 1974 empirisch belegt ist. Seit 1975 hat das FRS der Stabilisierung der Zinssätze einen geringeren Wert zugemessen als im Untersuchungszeitraum (vgl. Hein (1981), S. 10), obwohl inm seit 1977 neben anderen Zielen auch das Ziel der Stabilisierung des Zinsniveaus gesetzlich vorgegeben ist.

4) Die anhand von Quartalswerten getesteten Reaktionsfunktionen der Zentralbank enthielten auch die Anderung der Emissionsrendite festverzinslicher Wertpapiere als unabhängige Variable, die jedoch keinen statistisch signifikanten Einfluß auf Anderungen von $M_{1}$ ausübte. Vgl. die Schätzergebnisse im Anhang, Punkt 3. Auch bei Verwendung der Anderung der saisonbereinigten Zentralbankgeldmenge zu konstanten Reservesätzen konnte kein statistisch signifikanter Einfluß der Anderung der Emissionsrendite festverzinslicher Wertpapiere festgestellt werden. 
VI. ZUSAMMENFASSENDE WORDIGUNG DER NEOINSTITUTIONAL ISTISCHEN KRITIK

Die neoinstitutionalistische Kritik hebt zunächst auf die Wirkung marginaler Budgetsalden des Staates auf das Nutzenniveau der Individuen ab und postuliert eine Nettonutzensteigerung durch Budgetdefizite und eine Nettonutzenminderung durch Budgetüberschüsse, die vor allem dadurch bedingt sind, daB die negativen (positiven) Effekte von Budgetdefiziten (Budgetüberschüssen) in der Zukunft liegen und von den Individuen nicht wahrgenommen werden. Die genannten Sachverhalte führen in einer Demokratie, in der Politiker von den Wählern abhängig sind, zu einer Ausdehnung staatlicher Budgetdefizite, da die Wähler politischen Druck für die Erhöhung marginaler Budgetdefizite und gegen die Bildung marginaler Budgetuiberschüsse ausuben. Da die oberzeugung der okonomen und Politiker von der keynesianischen Theorie zu einer Aufhebung der Regel des Budgetausgleichs geführt hat, wird den Politikern die Erfüllung der Wählerforderungen ermöglicht. Die Folge sind wachsende Budgetdefizite und ein steigender Staatsanteil am Bruttosozialprodukt. Letzterer steigt insbesondere deshalb, weil Budgetdefizite den Steuerpreis öffentlicher Leistungen senken und dadurch eine Mehrnachfrage nach öffentlichen Gütern hervorrufen.

Eine exakte Analyse dieser Argumentationskette macht zunächst deutlich, daB BW bei ihrer Analyse nicht alle Effekte marginaler Budgetsalden auf das Nutzenniveau der Wähler berücksichtigt haben, und $d a B$ es sehr wohl negative Effekte gibt, die von den Wählern wahrgenommen werden. Hierbe $i$ ist vor allem an "psychologische" Grenzen der Staatsverschuldung ${ }^{1)}$ zu denken.

Im Hinblick auf die Hypothese der defizitbedingten Senkung des Steuerpreises für öffentliche Güter und der dadurch hervorgerufenen Ausdehnung des Staatsanteils am Sozialprodukt muß bemerkt wer-

1) Vgl. Abschnitt III.1.4.10. 
den, daß diese "Fiskalillusion" zwar theoretisch einen Prozeß in Gang setzen kann, der den Staatsanteil bis zum Erreichen eines neuen Gleichgewichts ständig erhöht. Angesichts der empirisch festgesteliten Steuerpreiselastizitäten der Nachfrage nach öffentlichen Gïtern ist dieser Effekt jedoch vergleichsweise gering, so daß die $B W-T h e s e$ gegenüber anderen neueren Erklärungsansätzen als weniger bedeutsam eingestuft werden muß.

Auch die von den Autoren postulierte Inflationswirkung von Budgetdefiziten muß äußerst skeptisch beurteilt werden, sowohl was die Verhältnisse in den USA als auch was die Bundesrepublik betrifft. Das Auftreten von Crowding-out-Effekten kann zwar a priori nicht ausgeschlossen werden. Die aus Gründen der Zinsstabilisierung resultierende Geldmengenausweitung war aber in den USA nicht so stark, daß sie zur Inflation führte. In der Bundesrepublik läßt sich nicht einmal eine Geldmengenwirkung statiicher Budgetdefizite empirisch belegen.

Die meisten der zunächst sehr plausibel klingenden neoinstitutionalistischen Hypothesen und die mit ihnen verbundene Anklage des Keynesianismus sind daher zu relativieren. Den Neoinstitutionalisten bleibt jedoch das Verdient, den bisher vernachlässigten Zusammenhang von Politik und makroökonomischen Entscheidungen herausgearbeitet und darauf aufbauend die entscheidende Bedeutung von Institutionen fur das Ergebnis fiskalischer Stabilisierungsmaßnahmen betont zu haben. Diese Bedeutung zeigt sich nicht nur darin, daB die Abschaffung der Regel des Budgetausgleichs das Wachstum der Staatsausgaben erleichtert hat. Vor allem der im Rahmen der Analyse der Fiskalillusion diskutierte EinfluB unterschiedlicher Abstimmungsregeln auf das Niveau der öffentlichen Ausgaben ${ }^{1)}$ sowie auch und gerade die Tatsache, daB die Pflicht der amerikanischen Zentralbank zur Stabilisierung des Zinsniveaus andere Ergebnisse hervorruft als die gesetzliche Verpflichtung der Deutschen Bundes-

1) Vgl. hierzu Pommerehne/Schneider (1978) 
bank zur Stabilisierung des Preisniveaus, erhärten die zentrale neoinstitutionalistische These, daB "INSTITUTIONS MATTER"1).

1) Buchanan/Wagner (1977), S. 144 
VII. ZUR THERAPIE: BEURTEILUNG EINER ROCKKEHR ZUR REGEL DES BUDGETAUSGLEICHS

\section{Die Budgetausgleichsregel aus neoinstitutionalistischer Sicht}

Im Rahmen dieser Arbeit wurde gezeigt, daB die Institutionen das Ergebnis ökonomischer Prozesse entscheidend beeinflussen können. Die von BW vorgeschlagene Therapie zur Beseitigung der im Gefolge keynesianischer Stabilisierungspolitik entstandenen Probleme liegt daher auf der Hand. Es müssen die Regeln, denen die Politiker bei ihren fiskalischen Entscheidungen unterworfen sind, verändert werden. In diesem Sinne wird vorgeschlagen, die Möglichkeit der Defizitfinanzierung durch Wiedereinfihrung der Regel des Budgetaus gleichs völlig abzuschaffen. Nur in Ausnahmefällen, in Situationen nationalen Notstands, sollen Defizite noch möglich sein 1). Damit die vorgeschlagene Regeländerung auf Dauer Guiltigkeit hat und von der jeweiligen Regierungspartei nicht nach Belieben geändert werden kann, soll die Regel des Budgetausgleichs Verfassungsrang erhat $\operatorname{ten}^{2)}$.

Die Regelung kommt nach Ansicht von BW nicht zuletzt den Politikern selbst entgegen, da sie sich gegenüber den Wünschen ihrer Wähler nach Mehrausgaben bzw. Steuersenkungen auf die in der Verfassung festgelegte Regelung berufen können ${ }^{3)}$. Damit ist allerdings noch nicht gesagt, daß der Staatsanteil nicht steigen könnte. Höhere Ausgaben sind nunmehr jedoch mit einer höheren Besteuerung verbunden, und die tatsächlichen Kosten öffentlicher Leistungen werden damit Wählern und Politikern ins BewuBtsein gerufen. Die Illusion, öffentliche Güter umsonst bereitstellen zu können, ent-

1) Vgl. Buchanan/Wagner (1977), S. $176 \mathrm{f}$.

2) $\mathrm{Vgl}$. ebenda, S. $175 \mathrm{f}$. "Budgets cannot be left adrift in the sea of democratic politics." (Ebenda, S. 175)

3) $\mathrm{Vgl}$. ebenda, S. 175 
fällt ${ }^{1)}$. Gleichzeitig wird die defizitbedingte Erhöhung der Inflationsrate gebremst ${ }^{2}$ ).

Das verfassungsmäßige Gebot einer Regel des Budgetausgleichs reicht aber allein zur Behebung der aufgezeigten Probleme noch nicht aus. Treten z.B. unerwartete Einnahmeausfälle oder Ausgabenzuwächse auf, so muß die Regel des Budgetausgleichs durch eine Regel ergänzt werden, die vorschreibt, wie die beiden Seiten des Budgets wieder zum Ausgleich gebracht werden.

Grundsätzlich kommen hierfür eine verfassungsmäßig fixierte automatische Anpassung der Einnahmen, der Ausgaben oder eine Kombination von Einnahmen- und Ausgabenanpassung in Betracht ${ }^{3}$. Obwoh 1 BW die Art der Anpassung für weniger wichtig halten als die verfassungsmäBige Vorschrift eines Anpassungsmechanismus per se, treten sie für eine automatische Anpassung der Ausgaben ein ${ }^{4}$ ). Im Falle eines potentiellen Budgetdefizits sollen sämtliche Ausgaben um denselben Prozentsatz gesenkt werden, wobei bestimmte Budgetkomponenten von der Reduktion ausgenommen werden können ${ }^{5}$ ).

Die Regel des Budgetausgleichs soll durch eine verfassungsmäßig vorgeschriebene Geldmengenregel ergänzt werden. Zur Stabilisierung des Preisniveaus soll das Geldmengenwachstum auf die Wachstumsrate des realen Sozialprodukts abgestimmt werden ${ }^{6)}$.

1) Vg1. Buchanan/Wagner (1977), S. 178

2) $\mathrm{Vgl}$. ebenda, S. 177

3) Vgl. ebenda, S. 178

4) Zur Begruindung wird lediglich angegeben "that the principles of sound finance are essentially the same for governments and persons". Ebenda, S. 179

5) Vgl. ebenda

6) In Jahren, in denen sich ein Budgetüberschuß abzeichnet, soll die Geldmengenausweitung durch den Kauf zur Rückzahlung anstehender Staatsschuldtitel durch das FRS geschehen. $\mathrm{Vgl}$. ebenda, S. $181 \mathrm{f}$. 


\section{Der spezifische Vorschlag von BUCHANAN/WAGNER ${ }^{1)}$}

Für die USA fordern BW die folgende Verfassungsänderung ${ }^{2}$ :

1. Der Präsident und der KongreB der Vereinigten Staaten müssen alljährlich ein ausgeglichenes Budget verabschieden.

2. Sollte aufgrund falscher Steuer-oder Ausgabenprognosen ein Budgetdefizit drohen, so sind die Ausgaben innerhalb von drei Monaten automatisch zu reduzieren. Etwaige Budgetüberschüsse sind zur Rückzahlung öffentlicher Schulden zu verwenden.

3. Diese Verfassungsänderung ist im Laufe der nächsten fünf Jahre nach ihrem Inkrafttreten in die Praxis umzusetzen. Während dieser obergangsperiode sind die Budgetdefizite jährlich um mindestens $20 \%$ ihres derzeitigen Standes zu reduzieren.

4. Abweichungen von dieser Regelung sind nur in Zeiten nationalen Notstands möglich, der durch beide Häuser des Kongresses mit Zwei-Drittel-Mehrheit unter Zustimmung des Präsidenten erklärt wird. Die Erklärung des nationalen Notstands erlischt innerhalb eines Jahres.

1) Vgl. Buchanan/Wagner (1977), S. 180. Ein alternativer Vorschlag des Federal Amendment Drafting Committee ( $v g l$. den Abdruck des Vorschlags bei Wildavsky (1980), S. $127 \mathrm{ff.}$ ) sieht eine verfassungsmäßige Begrenzung des Staatsausgabenwachstums durch das Wachstum des nominalen Bruttosozialprodukts vor, wobei eine mögliche inflationäre Aufblähung des Sozialprodukts durch die Regierung bestraft wird. Für den Fall, daß die Inflationsrate drei Prozent übersteigt, ist die erlaubte Steigerungsrate der Staatsausgaben um ein Viertel der drei Prozent übersteigenden Inflationsrate zu kürzen. Der Alternativvorschlag ist jedoch vom Ansatz her nicht mit dem Buchanan/Wagner-Vorschlag zu vergleichen. Während letzterer vorwiegend durch defizitbedingte alzokative und stabilisatorische Fehlentwicklungen begründet wird, setzt der Alternativvorschlag an der mit zunehmender Größe des Staatssektors sinkenden Qualität öffentlicher Leistungen und damit an der sogenannten X-Ineffizienz an. Vgl. vor allem Wildavsky (1980), S. 26 ff.

2) $\mathrm{Vgl}$. zu einem entsprechenden Vorschlag für das Vereinigte Königreich Buchanan/Burton/Wagner (1978a), S. $81 \mathrm{ff}$. 
3. Beurteilung des Vorschlags von BUCHANAN/WAGNER

Eine Beurteilung der von $B W$ vorgesehenen Verfassungsänderung setzt Beurteilungskriterien voraus. BUCHANAN selbst hat für die Beurteilung der Effizienz von Regeln das Kriterium des "agreement" aufgestellt' ${ }^{1)}$. Er nimmt dabei auf die Individuen einer Volkswirtschaft Bezug, die sich auf konstitutionelle Regeln einigen mussen, wobei ihnen die Marktbedingungen und die Verhaltensweisen der Politiker und Wirtschaftssubjekte, nicht aber die eigene Position in der nachkonstitutionellen Phase bekannt sind. Diejenigen Regeln, auf die sich die Individuen unter den genannten Bedingungen einstimmig einigen können, sind effizient ${ }^{2}$ ).

Da die potentiellen nachkonstitutionellen Auswirkungen alternativer Regeln die konstitutionelle Entscheidung über diese Regeln beeinflussen ${ }^{3)}$, ist im folgenden zu untersuchen, wie sich alternative Budgetregeln auf die Erreichung der Allokations- und Stabilisierungszielsetzung auswirken ${ }^{4}$. Erst auf dieser Grundlage kann eine Entscheidung über die optimale Budgetregel getroffen werden. Zuvor sollen jedoch noch einige Bemerkungen zu den Realisierungschancen der von $B W$ geforderten Verfassungsänderung eingefügt werden.

1) Vgl. Z.B. Buchanan (1975), S. 116

2) Vgl. Brennan/Buchanan (1980), S. $1 \mathrm{ff}$. Die Situation ist vergleichbar mit der Einigung auf die Regeln eines Kartenspiels, bei dem die Spieler zwar wissen, welche Karten sie beim späteren Spiel bekommen können, nicht aber, welche Karten sie tatsächlich bekommen. $V g l$. auch ebenda, S. 3

3) D.h. die von den Individuen vorausgesehenen Ergebnisse der Entscheidungen innerhalb alternativer Regeln in der nachkonstitutionellen Phase bestimmen ihre konstitutionelle Entscheidung über die optimale Regel.

4) Auf eine Analyse der Distributionseffekte der vorgeschlagenen Verfassungsänderung wird verzichtet, da die zum Defizitabbau in der Obergangsperiode geforderten Maßnahmen von BW nicht konkretisiert werden. Im übrigen sind die Determinanten der Inzidenz öffentlicher Ausgaben (vgl. Aaron/McGuire (1970), S. 914) empirisch kaum zu ermitteln. Alternative Hypothesen uber die Verteilungswirkungen öffentlicher Ausgaben lassen sich nur bei Zugrundelegung alternativer Grenznutzenelastizitäten des Einkommens empirisch bestätigen. $\mathrm{Vgl}$. hierzu Pommerehne (1975), S. $67 \mathrm{ff}$. Aus diesem Grund läBt sich auch die Inzidenz der im Fall von Prognosefehlern vorgesehenen proportionalen Ausgabenkürzung nicht beurteilen. 
3.1. Realisierungschancen und De-facto-Bindung der Politiker

Zunächst stellt sich die Frage, ob die Politiker einer derartigen Selbstbindung überhaupt zustimmen werden. Das Argument, die Politiker könnten sich bei der Realisierung des Vorschlags gegenüber Wählern, die marginale Defizite fordern, auf die Verfassung berufen und sich dadurch vom politischen Druck der Wähler frei machen, ist zwar a priori nicht von der Hand zu weisen. Andererseits mussen die Politiker aber damit rechnen, trotzdem für die Nicht-Realisierung von Ausgabenerhöhungen oder Steuersenkungsprogrammen verantwortlich gemacht zu werden. Akzeptiert man die neoinstitutionalistische Analyse der Kosten und Nutzen marginaler Budgetsalden, so ist und bleibt die Defizitfinanzierung ein bequemes Mittel, Belonnungen zu verteilen, ohne gleichzeitig belasten zu mussen. Es ist daher sehr fraglich, ob eigennutzige Politiker freiwillig auf dieses Mittel verzichten werden.

Die Realisierungschancen eines derartigen Vorschlags könnten jedoch aufgrund der in III.1.4.10. diskutierten psychologischen Effekte steigen. Wächst das Konsolidierungsbewußtsein der Bevölkerung mit steigenden Staatsdefiziten, so wird sich kaum ein wähler der Forderung nach Konsolidierung per se widersetzen. Solange eigene Begünstigungen nicht tangiert werden, wird jeder Wähler einer Konsolidierung der öffentlichen Finanzen zustimmen. Mit zunehmender Verschärfung der Haushaltsprobleme könnte somit politischer Druck im Hinblick auf eine verstärkte Konsolidierung die Realisierung des BW-Vorschlags begünstigen. Die Frage nach der Stärke des politischen Drucks duirfte jedoch entscheidend davon abhängen, inwieweit eine Mehrheit der Wähler im Rahmen der Konsolidierung mit dem Abbau eigener Beguinstigungen rechnet. A priori kann diese Frage nicht entschieden werden.

Wenn der BW-Vorschlag realisiert würde, bleibt die Frage, inwieweit die postulierte Zwei-Drittel-klausel eine effektive Schranke 
für die Politiker darstellen würde ${ }^{1)}$. Angesichts der Mehrheitsverhältnisse in den meisten westlichen Industriestaaten scheint die geforderte Zwei-Drittel-Mehrheit jedoch ausreichend, um einen Mißbrauch auszuschließen. Keine Opposition würde es der Regierung gestatten, zur Defizitalternative zu greifen, wenn nicht ein wirklicher Notstand vorliegt. D.h. man kann davon ausgehen, daß das Mehrheitserfordernis den gewüschten Effekt haben wird ${ }^{2}$.

\subsection{Allokative Effizienz}

Akzeptiert man die These, daß die Wähler ihre Forderungen nach öffentlichen Gütern von ihrem individuellen Steuerpreis abhängig machen, und daB Defizite die am wenigsten "merkliche" staatliche Einnahmequelle sind, so bringt die von BW vorgeschlagene Budgetausgleichsregel allokative Vorteile. Die Fiskalillusion und die mit ihr verbundene allokativ ineffiziente Erhöhung des Staatsanteils am Sozialprodukt werden reduziert.

Ahnliche Oberlegungen gelten unter der alternativen Annahme, daß die Wähler marginale Erhöhungen der öffentlichen Ausgaben als unabhängig von ihrer individuellen marginalen Steuerbelastung ansehen. Unter einer Regel des Budgetausgleichs können Politiker bei gegebenem Steueraufkommen nicht mehr bestimmte Ausgaben erhöhen, ohne gleichzeitig andere Ausgaben zu kürzen. Bei variablem Steueraufkommen müßten Ausgabenerhöhungen mit Steuererhöhungen verbunden werden. Das allokativ ineffiziente Wachstum des Staatsanteils könnte somit zumindest tendenziell begrenzt werden, da die Möglichkeiten der Politiker, Bürger zu begünstigen, ohne sie zugleich zu belasten, beschränkt werden.

1) Vgl. auch Tobin (1978), S. 622

2) Auf der anderen Seite stellt sich die Frage, ob durch diese Klausel nicht objektiv notwendige Defizite verhindert werden können. 
Dennoch ist die Regel des Budgetausgleichs unter allokativen Gesichtspunkten nicht generell zu befürworten, da die genannten Allokationsvorteile nur bei Gütern auftreten, deren Nutzenstiftung auf die Gegenwart begrenzt ist. Im Falle der Bereitstellung von öffentlichen Gütern, die auch in zukünftigen Perioden Nutzen stiften ${ }^{1}$ ), kann die ständige Realisierung eines materiellen Budgetausgleichs zu allokativen Verzerrungen führen.

Diese These soll durch einige Oberlegungen zur allokativen Effizienz von Budgetsalden verdeutlicht werden ${ }^{2}$. Es geht im folgenden um die Frage, welcher Budgetsaldo unter den von BW getroffenen Annahmen über das Verhalten von Politikern ${ }^{3)}$ zu einer allokativ effizienten Bereitstellung öffentlicher Investitionsgüter führt, wenn weiterhin unterstellt wird, daß die Entscheidungen der Individuen über die von ihnen gewünschte Menge an öffentlichen Gütern von den wahrgenommenen Kosten und Nutzen dieser Güter abhängt ${ }^{4}$. Die Analyse geht von der einschränkenden Annahme eines vollkommenen Kapitalmarktes aus, um dann schrittweise zu einer realistischeren Betrachtungswe ise überzugehen.

Im theoretischen Grenzfall eines vollkommenen Kapitalmarktes, der neben vollkommener Voraussicht die Gleichheit von Soll-und Habenzinssatz mit der marginalen Zeitpräferenzrate der Individuen impliziert, hat die Art der Finanzierung keinen EinfluB auf die effiziente Versorgung mit öffentlichen Investitionsgütern. Bei vollständiger Steuerfinanzierung des Investitionsgutes in der Gegen-

1) Diese Güter seien im folgenden als öffentliche Investitionsgüter bezeichnet (vgl. Wissenschaftlicher Beirat (1980), S. 31), während öffentliche Guiter, die sämtliche Nutzungen in der Gegenwart abgeben, als öffentliche Konsumgüter bezeichnet werden.

2) Vgl. auch Buchanan (1967), S. $262 \mathrm{ff}$.

3) Aus Gründen der Anschaulichkeit seien wieder nutzenmaximierende, aber von den augenblicklichen Wählerwünschen abhängige Politiker unterstellt. Vgl. Abschnitt III.1.3.2.

4) $\mathrm{Vgl}$. auch Abschnitt III.1.4.2. Die gesamte Analyse unterstellt weiterhin, daB Budgetdefizite durch Ausgabe von Staatsschuldtiteln finanziert werden. 
wart können die besteuerten Individuen einen Kredit aufnehmen und diesen entsprechend der Nutzung des Investitionsgutes zurückzahlen. Der für das Investitionsgut in der Gegenwart zu zahlende Steuerpreis entspricht in diesem Fall dem Gegenwartswert der für den Kredit zu zahlenden Zins- und Tilgungsbeträge.

Sobald jedoch die Annahme der vollständigen Voraussicht aufgegeben wird ${ }^{1)}$, bewirkt eine Regel des jährlichen materiellen Budgetausgleichs eine Erhöhung des subjektiv wahrgenommenen Steuerpreises von Investitionsgütern, da die Individuen nicht mehr alle zukünftigen Nutzungsabgaben dieser Güter in ihren Kalkül einbeziehen. Ein rational unvollständig informiertes Individuum wird daher unter einer Regel des Budgetausgleichs tendenziell zu wenig öffentliche Investitionsgüter nachfragen.

Die von BW aufgestellte Hypothese der unvollständigen Berücksichtigung zukünftiger Effekte bei Wählerentscheidungen, mit der die Autoren im Falle der Defizitfinanzierung eine illusionsbedingte oberversorgung mit öffentlichen Konsumgütern begründen, bedingt somit im Falle eines jährlichen materiellen Budgetausgleichs eine Unterversorgung mit öffentlichen Investitionsgütern. BUCHANAN selbst weist in einer fruheren Publikation explizit auf diesen Effekt $\operatorname{hin}^{2}$ ).

Allokative Effizienz erfordert demnach gerade bei Zugrundelegung der von BW postulierten Wahrnehmungsasymmetrien ${ }^{3)}$ eine Defizitfinanzierung solcher Güter, deren Nutzen in der Zukunft anfällt. Die fehlende Wahrnehmung der zukünftigen Nutzen dieser Güter muB durch

1) Die Annahme Sollzinssatz = Zeitpräferenz wird zur Verdeutlichung der grundsätzlichen Zusammenhänge vorläufig beibehalten.

2) Vgl. Buchanan (1967), S. 264 f.

3) Vgl. Abschnitt III.1.4.3. sowie Buchanan/Wagner (1977), S. 100 
die fehlende Wahrnehmung der mit Defiziten verbundenen zukünftigen Steuerverbindlichkeiten kompensiert werden ${ }^{1}$ ).

Die Höhe des zur Bereitstellung einer optimalen Menge öffentlicher Investitionsgüter erforderlichen Defizits hängt somit davon ab, inwieweit zukünftige Nutzen und zukünftige Steuerverbindlichkeiten von den Individuen wahrgenommen werden.

Unter der einschränkenden Annahme, daß von den gesamten Kosten und Nutzen eines Investitionsgutes zum Zeitpunkt $t_{0}$ die in der ersten Periode auftretenden Kosten- und Nutzenanteile vollständig, die in den darauffolgenden Perioden auftretenden aber überhaup.t nicht wahrgenommen werden, wäre demnach ein Budgetdefizit optimal, dessen Höhe den Gesamtkosten der geplanten Investitionsobjekte abzüglich der in der ersten Periode anfallenden Nutzen entspricht. Die Höhe des optimalen Defizits sinkt mit steigender Wahrnehmung des zukünftigen Nutzens von Investitionsobjekten ${ }^{2)}$. Geht man davon aus, daß zukünftige Nutzen von Investitionen umso weniger wahrgenommen werden, je weiter sie in der Zukunft liegen, so ist das optimale Haushaltsdefizit umso höher, je größer der Anteil langfristiger Investitionsobjekte am Gesamtbudget ist.

Wird zusätzlich die Annahme der Obereinstimmung von Sollzinssatz und marginaler Zeitpräferenz aufgegeben, so steigen die opportunitätskosten von Gütern, deren Nutzen in der Zukunft anfäl1t. Soll

1) Die "public debt illusion" auf der Einnahmenseite muß die sogenannte "asset illusion" auf der Ausgabenseite kompensieren. Vgl. Buchanan (1967), S. 264 f. Vgl. hierzu schon McCulloch (1851), S. 281 (zitiert nach West/Winer (1980), S. 607), der eine Finanzierung von öffentlichen Gütern über unmerkliche Steuern als legitim ansieht, da die Nutzen öffentlicher Güter von den Steuerzahlern nicht vollständig wahrgenommen werden. Im folgenden sei jedoch zur Vereinfachung unterstellt, daß sämtliche in der Budgetperiode zu entrichtenden Steuern vollständig wahrgenommen werden. Der allgemeine Fall einer für die Regierung optimalen Fiskalillusion bei Budgetausgleich wird von West/Winer (1980), S. $608 \mathrm{ff}$. diskutiert.

2) Sie steigt, wenn zukünftige Steuerverbindlichkeiten stärker wahrgenommen werden als zukünftige Nutzen. 
weiterhin eine der Zeitpräferenz der Individuen entsprechende Menge von Investitionsgütern bereitgestellt werden, so steigt das optimale Defizit, da der von den Individuen wahrgenommene Steuerpreis gesenkt werden muB ${ }^{1}$ ).

Akzeptiert man daher die von BW postulierten Wahrnehmungsdiskrepanzen, so muB eine verfassungsmäBige Budgetregel ein Defizit vorsehen ${ }^{2)}$, dessen Höhe mit sinkendem Ausmaß der Wahrnehmung aukünftiger Nutzen von Investitionsgütern und mit dem Grad der Unvolzkommenheit der Kapitalmärkte zunimmt. Dieses Defizit muB in den Folgeperioden entsprechend dem "Pay-as-you-use-Prinzip"3) verzinst und getilgt werden. Die praktische Durchführung des "Pay-as-you-use-Prinzips" setzt eine Finanzierungsrechnung für statliche Investitionen und als deren Grundlage eine Prognose der zeitlichen Nutzungsabgabe staatlicher Investitionsgüter voraus. Gerade auf diesen Punkt kann nicht verzichtet werden, da eine Defizitfinanzierung von Investitionsgitern per se noch keine allokativen Vorteile bewirkt. Letztere treten nur auf, wenn eine annähernd exakte obereinstimmung der Nutzungsabgabe der Investitionsobjekte mit der Laufzeitenstruktur der zu ihrer Finanzierung emittierten Wertpapiere gesichert ist. Eine am Allokationsziel orientierte Budgetregel muB daher eine Finanzie-

1) Defizitvermindernd müssen sich möglicherweise die von BW angedeuteten Crowdingout-Effekte von Budgetdefiziten auswirken (vgl. hierzu die Abschnitte V.1. und V.2.1.). Trifft die These $z u$, daß staatliche Budgetdefizite zu einem crowdingout privater Investitionen führen und weisen öffentliche Investitionen eine geringere Produktivität auf als private, so ist den erörterten positiven Allokationseffekten einer Defizitfinanzierung staatlicher Investitionen ein negativer Allokationseffekt entgegengerichtet. In diesem Fall müBten die beiden entgegengerichteten Effekte gegenübergesteilt und e in Optimum bestimmt werden. Da Crowding-out-Effekte jedoch nicht in sämtlichen Konjunktursituationen auftreten, ergibt sich nur zeitweise eine Modifikation der bisher abgeleiteten Ergebnisse, welche auf konstitutionellem Niveau nicht bericksichtigt und stattdessen einer nachkonstitutionellen politischen Entscheidung unterworfen werden sollte.

2) "The analysis suggests, therefore, that public debt issue may be chosen as an appropriate part of the over-all 'constitution' of a fiscal structure, provided that limitations are imposed to insure that debt financing be restricted to projects that yield benefits over time." Buchanan (1967), S. 265

3) Vgl. Musgrave (1958), S. 73 f. sowie Tolkemitt (1975), S. 25 ff. 
rungsrechnung für staatliche Investitionen, die auf der Basis einer Prognose der zeitichen Nutzungsabgabe defizitfinanzierter Investitionsgüter eine Ubereinstimmung der zeitlichen Nutzungsabgabe der Investitionen mit der Laufzeitenstruktur der zu ihrer Finanzierung aufgenommenen Kredite sicherstellt, zwingend vorschreiben.

Bei der praktischen Anwendung dieser Oberlegungen treten neben der Schwierigkeit einer exakten Prognose der zukünftigen Nutzungsabgabe von Investitionsgütern selbstverständlich auch die Probleme der exakten Ermittlung der Wahrnehmungsdiskrepanzen und der Bestimmung des Grades der Unvollkommenheit der Kapitalmärkte auf. Eine konstitutionelle Regel wird daher eine vollständige Defizitfinanzierung nur für Investitionsgüter mit sehr langer Nutzungsdauer vorschreiben. Im Falle von Gütern mit mittlerer Nutzungsdauer muB im konkreten Einzelfall eine nachkonstitutionelle politische Finanzierungsentscheidung getroffen werden, wobei die Bildung von Defiziten jedoch, um einen MiBbrauch seitens der Regierung auszuschlieBen, durch das Erfordernis einer Zwei-Drittel-Mehrheit erschwert werden sollte.

Die Trennung zwischen "sehr langfristigen" und kürzerfristigen Investitionen ist indessen in der Praxis nicht ohne Probleme und muß auf der Grundlage empirischer Untersuchungen des Zeithorizonts und der Zeitpräferenz der Wähler erfolgen. Als "sehr langfristig" muB ein Investitionsgut bezeichnet werden, bei dem die empirische Analyse zu dem Ergebnis gelangt, daß seine zukünftigen Nutzungen aufgrund der Länge seiner Nutzungsdauer von den Wählern nicht mehr wahrgenommen werden. Letztendlich muß aus Grüden der praktischen Durchführbarkeit auch in diesem Bereich auf der Basis empirischer Untersuchungen eine politische (Verfassungs-) Entscheidung darüber fallen, von welcher Nutzungsdauer an Investitionen als sehr langfristig zu bezeichnen sind.

Zusammenfassend $1 a ̈ B t$ sich feststellen, daß allokative Oberlegungen bei Annahme abhängiger Politiker und der von BW postulierten Wahrnehmungsdiskrepanzen zu einer Ablehnung des von den Autoren 
geforderten jährlichen Budgetausgleichs führen. Allokative Effizienz erfordert gerade bei Zugrundelegung der von BW getroffenen Annahmen ein bestimmtes $A$ usmaB der Defizitfinanzierung von Gütern, deren Nutzen in der Zukunft anfallen. Das exakte Ausmaß der Defizitfinanzierung von Investitionsgütern kann auf konstitutioneller Ebene nicht festgelegt werden und bedarf daher in bestimmten Fä1len einer nachkonstitutionellen politischen Entscheidung. Die Verzinsung und Tilgung der aufgenommenen Kredite muß entsprechend dem "Pay-as-you-use-Prinzip" erfolgen, d.h. die Laufzeitenstruktur der Kredite ist auf die zeitliche Nutzungsabgabe der defizitfinanzierten Investitionsgüter abzustimmen.

\subsection{Stabilisatorische Effizienz}

Nach Ansicht von BW stellt die Rückkehr zur Regel des Budgetausgleichs verbunden mit einer Geldmengenregel auch eine geeignete MaBnahme zur Inflationsbekämpfung dar. Das Beschäftigungsziel spielt indessen in der Argumentation der Autoren nur eine untergeordnete Rolle. Es wird zwar als politisches Ziel anerkannt ${ }^{1}$, die Problematik der Arbeitslosigkeit wird jedoch relativiert. BW gehen von der Stabilität der Marktwirtschaft ${ }^{2}$ aus und sehen Arbeitslosigkeit als vorubergehendes Problem an, welches ohne Eingriffe des Staates durch Marktkräfte behoben werden kann ${ }^{3)}$.

1) Vgl. Buchanan/Wagner (1977), S. 171

2) Vgl. ebenda, Kapitel 11 sowie Buchanan/Wagner (1978), S. 635

3) "Nonetheless, all such instances of involuntary unemployment will tend to be, ... of short duration, and they would tend to occur fairly evenly over time." Buchanan/Wagner (1977), S. 172. In der schon erwähnten früheren Publikation "Public Finance in Democratic Process" räumt Buchanan dagegen die Notwendigkeit der Berücksichtigung einer aktiven Beschäftigungspolitik in der Finanzverfassung ein: "The individual may,... 'vote for' the authorization for the government to create deficits deliberately during periods of threatened retardation in aggregate community income growth." Buchanan (1967), S. 269. Allerdings wird - unter Bezugnahme auf die "keynesianische" Situation tiefer Depression - eine Geldschöpfungsfinanzierung der Defizite gefordert. Vgl. ebenda, S. $276 \mathrm{ff}$. 
Gerade im Fall einer konsequenten regelgebundenen Geldpolitik, die sich ohne Zweifel positiv auf die Erreichung des Ziels der Preisniveaustabilität auswirken würde, kann ein materiell ausgeglichenes Budget jedoch die durch exogene Störungen verursachten Beschäftigungsprobleme zumindest kurzfristig verschärfen, d.h. es sind negative Nebeneffekte denkbar, deren volkswirtschafti iche Kosten von $B W$ möglicherweise unterschätzt werden.

Diese u.a. von TOBIN ${ }^{1)}$ hervorgehobene Problematik der von BW vorgeschlagenen Anpassungspolitik läßt sich leicht anhand eines einfachen Kreislaufmodells demonstrieren.

Das Nettosozialprodukt setzt sich in einer geschlossenen Volkswirtschaft aus Konsum (C), Investitionen (I) und Staatsausgaben (A) zusammen

(VII.1) $\quad Y=C+I+A$.

Der Konsum sei gemäß einer (konstanten) marginalen Konsumquote $c$ vom verfügbaren Einkommen abhängig ${ }^{2}$ :

(VII.2) $\quad C=c(Y-T+T r)$.

Be $i$ einem einkommensabhängigen steueraufkommen ${ }^{3}$ )

(VII.3) $\quad T=\tau Y$

kann die Konsumfunktion demnach als

(VII.3a) $\quad C=c(Y-\tau Y+T r)$

1) Vgl. auch Tobin (1978), S. $624 \mathrm{f}$.

2) T repräsentiert das Steueraufkommen, $\mathrm{Tr}$ die als autonom angenommenen Transferausgaben.

3) $\tau$ stellt den effektiven Grenzsteuersatz dar und darf nicht mit dem im Einkommensteuergesetz festgelegten Marginalsatz verwechselt werden. $\tau$ gibt das Verhältnis der Erhöhung des gesamten Steueraufkommens zur Erhöhung des Volkseinkommens an. Vgl. Musgrave (1974), S. 473, Fußnote 2. 
geschrieben werden.

Die Investitionen seien autonom vorgegeben:

$(V I I .4) \quad I=I$.

Für Anderungen des Nettosozialprodukts läßt sich somit die Gleichung

(VII.5) $\quad \Delta Y=c(\Delta Y-\Delta T+\Delta T r)+\Delta I+\Delta A$

bzw.

(VII.5a) $\Delta Y=c \Delta Y-c \tau \Delta Y+c \Delta T r+\Delta I+\Delta A$

ableiten.

Welche Folgen hat der Vorschlag von BW in diesem Mode11, wenn die autonomen Investitionen plötzlich um $\Delta I$ sinken? Die kurzfristige Multiplikatorwirkung ergibt sich im Falle der Anpassung der staatlichen Realausgaben aus Gleichung (VII.5a) für $\Delta A=\Delta T=\tau \Delta Y$ als

(VII.6) $\quad \Delta Y=\frac{1}{(T-c)(T-\tau)^{\Delta I}}$.

Man erhält also das bekannte Ergebnis, daB die Anpassung der Realausgaben an die verminderten Steuereinnahmen, d.h. eine sogenannte Parallelpolitik, zyklusverstärkend wirkt ${ }^{1}$.

Wäre $A$ trotz des Einkommensrückgangs konstant gehalten worden $(\Delta A=0)$, so würde lediglich eine Einkommenssenkung von

(VII.7) $\quad \Delta Y=\frac{1}{1-c(1-\tau)^{\Delta I}}$

1) Vgl. z.B. Tobin (1978), S. $624 \mathrm{f}$. 
resultieren. In diesem Fall hätte nämlich die eingebaute Flexibilität des Steuersystems ein marginales Budgetdefizit erwirtschaftet, welches dem Kontraktionsprozeß entgegengewirkt hätte.

Werden indessen anstelle der Realausgaben die Transferausgaben an das rückläufige Steueraufkommen angepaßt $(\Delta T r=\Delta T=\tau \Delta Y)$, so resultiert aus (VII.5a)

(VII.8) $\Delta Y=\frac{1}{1-c} \Delta \bar{I}$,

d.h. die Ausgabensenkung wirkt nicht prozyklisch. Der Grund hierfür liegt darin, daß sich in diesem Fall die effektive marginale Konsumquote nicht ändert ${ }^{1}$ ).

Dasselbe gilt unter den gesetzten Annahmen für die von BW weniger präferierte Anpassung der Steuern an die konstanten Staatsausgaben mittels Steuersatzerhöhung. Wegen $\Delta T=0$ bleibt auch in diesem Fall die effektive marginale Konsumquote konstant ${ }^{2}$ ).

Das letztere Ergebnis gilt jedoch nur unter den zu Beginn gesetzten vereinfachenden Annahmen. Geht man realistischerweise davon aus, daB das Investitionsvolumen aufgrund der Steuersatzsteigerung zurückgehen wird, so muB auch bei einer Anpassung des Steueraufkommens an die Ausgaben mit einer Verschärfung des Abschwungs gerechnet werden.

Im gegebenen Modellzusammenhang erscheint daher eine automatische Anpassung der Transferausgaben als mit dem Stabilisierungsziel am ehesten vereinbar, doch können gerade bei dieser Möglichkeit Zielkonflikte mit dem Verteilungsziel auftreten. Eine Variation der staatlichen Transferzahlungen im Konjunkturverlauf ist daher weder

1) In der Konsumfunktion $C=c(Y-T+T r)$ ist $T=T r$, so daB in Gleichung (VII.5) $\Delta T r-\Delta T=0$ gilt.

2) Der Steuersatz steigt auf $\tau_{n}=\tau_{0} Y_{0} /\left[Y_{0}-(1 / 1-c) \Delta T\right]$. 
politisch durchsetzbar noch wünschenswert ${ }^{1)}$ und kommt als automatisches Anpassungsinstrument nicht in Frage. Geht man weiterhin realistischerweise davon aus, daß die Mehrzahl der Transferempfänger eine höhere marginale Konsumquote aufweist als der Durchschnitt der Bevölkerung, so ist auch bei Anpassung der Transferzahlungen mit einer Verschärfung des Konjunkturabschwungs zu rechnen.

Zumindest kurzfristig ist daher bei Realisierung des Vorschlags von BW mit erheblichen volkswirtschaftlichen Kosten in Form von Arbeitslosigkeit zu rechnen. Diese Aussage gilt jedenfalls so lange, bis Alternativen zur keynesianischen Politik der Nachfragesteuerung gefunden sind, welche die mit der vorgeschlagenen Anpassungspolitik verbundenen negativen Multiplikatoreffekte mildern oder gar verhindern ${ }^{2)}$. Hält man daher die Probleme der Arbeitslosigkeit für gravierender als BW, so muB die von den Autoren vorgeschlagene Verfassungsänderung abgelehnt oder zumindest modifiziert werden.

AbschlieBend scheinen einige Bemerkungen zu der von BW vorgeschlagenen Obergangsstrategie angebracht. Letztere sieht vor, im Laufe von funf Jahren durch Abbau des Budgetdefizits um jeweils $20 \%$ der derzeitigen Höhe zum Budgetausgleich zurückzukehren.

Diese Regel, die ökonomisch nicht begründet wird, erscheint angesichts der bisherigen Oberlegungen dieses Abschnitts zu starr und zu wenig konkret. Eine geeignete Obergangsstrategie muB darauf angelegt sein, das angestrebte Ziel mit möglichst geringen Reibungs-

1) Als krasses Beispiel können die Zahlungen der Arbeitslosenversicherung angeführt werden, die im Konjunkturabschwung fallen statt steigen müBten. Eine Kürzung dieser Zahlungen kann gerade in der Rezession nicht ernsthaft gefordert werden.

2) Zu denken ist vor allem an angebotsorientierte Konzepte. Diese zielen darauf $a b$, durch Steuererleichterungen für Unternehmen und durch eine Senkung der Einkommensteuer die Investitionen und die private Ersparnis zu erhöhen, woraus nach Ansicht der Verfechter dieser Konzepte langfristig ein ausgeglichenes Budget und eine niedrigere Inflationsrate resultiert. Derartige Ansätze sind jedoch empirisch noch nicht erprobt. Vgl. Dornbusch/Fischer (1978), S. $386 \mathrm{f}$. 
verlusten zu erreichen. Die in England unter Premierministerin Thatcher abrupt vollzogene Ablösung keynesianischer Wirtschaftspolitik durch monetaristische Konzepte, die zu einer erheblichen Verschärfung der Beschäftigungsprobleme geführt hat, liefert einen eindrucksvolien Beweis für die These, daß in der Vergangenheit aufgetretene gesamtwirtschaftliche Probleme kurzfristig ohne beträchtliche volkswirtschaftliche Kosten in Form von Arbeitslosigkeit kaum gehoben werden können.

Zwar braucht eine Konsolidierungsstrategie nicht notwendigerweise zu einem Konjunkturrückgang zu führen. Dabei können psychologische Effekte, die die marginale Konsumquote und die Investitionsneigung erhöhen, genauso eine Rolle spielen, wie gezielte Umstrukturierungen des Haushalts, die auf eine Substitution von wenig konjunkturwirksamen Haushaltspositionen durch solche mit hohen Investitionsanreizen gerichtet sind.

Voraussetzung einer längerfristig angelegten Konsolidierungsstrategie ist jedoch eine detaillierte Analyse der konjunkturellen Effekte einer Vielzahl staatlicher Einnahmen und Ausgaben ${ }^{1}$, so daB der Vorschlag von BW, obwohl er vom Grundgedanken her als durchaus positiver Impuls zu bewerten ist, in vielen Punkten nicht ausgereift erscheint. In der Tendenz ist daher TOBIN zuzustimmen: "The language leaves lots of loose ends. . It will take a few more hours or years at the economic and legal drawing boards before these proposals are ready for congress and the states ${ }^{2}$ )."

\subsection{Zusammenfassung}

Es wurde gezeigt, daB die Realisierung der von BW geforderten Verfassungsänderung, welche ein jährlich materiell ausgeglichenes Bud-

1) Ein erster Ansatz findet sich bei Signer (1980).

2) Tobin (1978), S. 624 
get vorsieht, zu einer allokativ ineffizienten Unterversorgung mit öffentlichen Investitionsgütern führen würde. Weiterhin besteht die Gefahr, daB die vorgeschlagene Regelung zu einer Verschärfung der Beschäftigungsprobleme führt. Solange Alternativkonzepte, die eine kurzfristige Milderung der Kosten der Arbeitslosigkeit bei Budgetausgleich erlauben, noch nicht entwickelt sind, muB die ständige Realisierung eines materiellen Budgetausgleichs somit auch aus Stabilisierungsgrüden abgelehnt werden.

Sieht man den Grundgedanken des Vorschlags von BW allerdings darin, Budgetdefizite auf das zur Lösung spezieller Zielsetzungen notwendige MaB zu begrenzen, so bietet es sich an, den spezifischen Vorschlag der Autoren nicht völlig abzulehnen, sondern zu modifizieren.

Der folgende Abschnitt VII.4. entwickelt daher für den Allokationsund Stabilisierungsbereich spezielle Ergänzungsregelungen, die im Rahmen der Wiedereinführung des materiellen Budgetausgleichs als grundsätzlicher Budgetregel berücksichtigt werden müssen, um negative Nebeneffekte auf das Allokations- und Stabilisierungsziel zu verhindern.

\section{Ein alternativer Vorschlag zur Anderung der Finanzverfassung}

Die bisherigen Ausführungen dieses Abschnitts zeigen, daB der Vorschlag von BW zur Anderung der Finanzverfassung zwar als beachtijcher Impuls zu werten ist, im Hinblick auf Stabilisierungs- und Allokationsprobleme aber einer Modifizierung bedarf. Eine Wiedereinführung des materiellen Budgetausgleichs als grundsätzlicher Budgetregel muB daher neben der von BW berücksichtigten allgemeinen Ausnahmeregelung, welcher z.B. im Falle eines Krieges Bedeutung zukommen könnte, spezielze, auf den Allokations-und Stabilisierungsbereich zugeschnittene ErgänzungsregeZungen vorsehen. 
Auf der Basis der Oberlegungen zur optimalen intertemporalen Allokation ist eine Kreditfinanzierung sehr langfristiger Investitionsobjekte als Regelfinanzierung ${ }^{1)}$ vorzusehen. Zusätzlich soll das Parlament jedes Jahr mit Zwei-Drittel-Mehrheit diejenigen kürzerfristigen Investitionsgüter festlegen, die über Kredite finanziert werden. Um eine "Pay-as-you-use"-Finanzierung sicherzustellen, ist außerdem die Obereinstimmung der zeitlichen Nutzungsabgabe der Investitionen mit der Laufzeitenstruktur der zu ihrer Finanzierung ausgegebenen Wertpapiere durch eine Finanzierungsrechnung nachzuwe isen.

Der Vorteil dieser Regelung besteht insbesondere darin, daB sie eine vollständige Steuerfinanzierung konsumtiver Staatsausgaben vorsieht. Die bisherige Möglichkeit der Kreditfinanzierung eines im Rahmen der Haushaltsplanung auftretenden Fehlbetrags ist damit ausgeschlossen ${ }^{2)}$. Das geforderte Zwei-Drittel-Quorum hat zur Folge, daB eine Nettoneuverschuldung angesichts der Mehrheitsverhältnisse in westlichen Demokratien von Teilen der Opposition mitgetragen werden muB, was einen MiBbrauch der Kreditfinanzierungsalternative durch die Regierungspartei erschwert. Das Mehrheitserfordernis könnte zwar dazu führen, daB die Opposition ökonomisch sinnvolle

1) Eine derartige Regel unterscheidet sich von der in Art. 115 GG vorgesehenen Vorschrift, gemäB der die Einnahmen aus Krediten durch die im Haushaltsplan veranschlagten Investitionen begrenzt werden, in zweierlei Hinsicht. Zum einen ist die in Art. $115 \mathrm{GG}$ gezogene Obergrenze in all den Fällen zu hoch, in denen Investitionen in der Budgetperiode bereits Nutzungen abgeben. Zum anderen kann einer Bestimmung, die lediglich eine Obergrenze für Defizite vorsieht, entgegengehàlten werden, daß sie in den Fällen, in denen die Obergrenze nicht ausgeschöpft wird, zu einer allokativen Ineffizienz führt. Der Grund liegt darin, da B allokative Effizienz, abgesehen von den diskutierten Modifikationen, die Gleichheit von Defiziten und Investitionen erfordert.

2) Problematisch bleibt weiterhin der Begriff der öffentlichen Investition, da nahezu jede öffentliche Ausgabe in der Zukunft Nutzungen abwirft. Vgl. Wissenschaftlicher Beirat (1980), S. 32. Besondere Probleme entstehen bei der Frage, ob Ausgaben für das Humankapital zu den öffentlichen Investitionen gezählt werden sollen. Der Wissenschaftliche Beirat beim Bundesministerium der Finanzen empfiehlt aufgrund von Erfassungs- und Zurechnungsproblemen eine Beschränkung des Investitionsbegriffs auf Sachinvestitionen. $\mathrm{Vgl}$. ebenda, S. $33 \mathrm{f}$. und S. 52 
Defizite aus politischen Gründen ablehnt. Es ist jedoch zu berücksichtigen, daB auch die Opposition nicht unabhängig von wählerwüschen ist, so daB sie sich nicht generell und ohne sachliche Begründung gegen eine Defizitfinanzierung kürzerfristiger Investitionsgüter sperren kann.

Eine spezielle Ergänzungsregel im Stabilisierungsbereich ist deshalb erforderlich, weil die von BW für Notsituationen vorgesehene allgemeine Ausnahmeregel eine Krisensituation voraussetzt, auf die die Politiker reagieren mussen. Da diese Reaktion neben der Zustimmung des Präsidenten eine Zwei-Drittel-Mehrheit im Senat und Repräsentantenhaus zur Bedingung macht, könnte der mit der politischen Diskussion verbundene Entscheidungs-Lag im Fall einer plötzlich auftretenden Beschäftigungskrise den rechtzeitigen Einsatz fiskalischer Instrumente verhindern.

Aus diesem Grund wird eine Regel gefordert, die Defizite dann erlaubt, wenn die Arbeitslosenquote einen bestimmten, vom Parlament mit Zwei-Drittel-Mehrheit zu Beginn der Haushaltsperiode festgesetzten Prozentsatz übersteigt. Dabei soll die zulässige Höhe der Nettoneuverschuldung - ebenfalls mit Zwei-Drittel-Mehrheit - an das AusmaB der Abweichung der tatsächlichen Arbeitslosenquote von der vom Parlament festgesetzten "kritischen" Arbeitslosenquote geknüpft werden.

Auch in diesem Fall hat das geforderte Zwei-Drittel-Quorum die Konsequenz, daß die Opposition dem festzulegenden Prozentsatz zustimmen muB. Die Möglichkeit der Regierungspartei, durch eine zu niedrige Festsetzung der kritischen Arbeitslosenquote Defizite nach Belieben machen zu können, ist daher ausgeschaltet. Andererseits kann die Opposition nicht auf einem zu hohen Prozentsatz bestehen, da sie sonst von der Regierung in der offentlichkeit für die Beschäftigungskrise verantwortlich gemacht wird. 
Auf der Basis dieser Oberlegungen ergibt sich für die Bundesrepublik Deutschland die folgende Anderung von Art. 115 GG:

(1) Der jährliche Haushalt des Bundes ist, abgesehen von den in Absatz (2) vorgesehenen Ausnahmeregelungen, materiell auszugleichen. Sollte im Laufe des Haushaltsjahres aufgrund von Fehlern bei der Prognose der Steuereinnahmen oder der Staatsausgaben ein Defizit drohen, so sind innerhalb der nächsten drei Monate Maßnahmen zur Realisierung des Haushaltsausgleichs zu ergreifen.

(2) Ausnahmen sind nur in folgenden Fällen möglich:

1. Wenn Bundestag und Bundesrat mit den Stimmen von zwei Dritteln ihrer Mitglieder eine Situation nationalen Notstands feststellen.

2. Wenn die Arbeitslosenquote einen vom Bundestag mit Zwei-Drittel-Mehrheit zu Beginn der Haushaltsperiode festgelegten Prozentsatz (kritische Arbeitslosenquote) übersteigt. Dabei ist das Ausma $B$ der Kredite zu Beginn der Haushaltsperiode mit zwe $i$ Dritteln der Stimmen des Bundestags an das AusmaB der Abweichung der Arbeitslosenquote von ihrem kritischen Wert zu knüpfen.

3. Langfristige ${ }^{1)}$ Investitionsobjekte sind unter Berücksichtigung der bereits in der Haushaltsperiode anfallenden Nutzungen über Krediteinnahmen zu finanzieren. Ober die Finanzierung kurzfristiger Investitionsobjekte entscheidet der Bundestag mit Zwei-Drittel-Mehrheit.

1) Erfolgt gleichzeitig mit dieser Verfassungsänderung eine Verfassungsentscheidung über die Frage, von welcher Nutzungsdauer an Investitionen als "langfristig" zu bezeichnen sind, so kann auch die entsprechende Nutzungsdauer in die Verfassung aufgenommen werden. 
(3) Werden Investitionsgüter über Defizite finanziert, so ist eine Obereinstimmung ihrer zeitlichen Nutzungsabgabe mit der Laufzeitenstruktur der zu ihrer Finanzierung aufgenommenen kredite durch eine Finanzierungsrechnung nachzuweisen. Die zeitliche Nutzungsabgabe der Investitionen ist im Rahmen der Haushaltsberatungen durch eine Prognoserechnung festzustellen.

(4) Das Nähere regelt ein Bundesgesetz ${ }^{1)}$.

1) Das Bundesgesetz muß Z.B. die Methode festlegen, anhand derer die Arbeitslosenquote ermittelt wird. 


\section{DER NEOINSTITUTIONALISTISCHE BEITRAG ZUR FINANZPOLITIK}

Die Analyse der Kritikpunkte von BW an der keynesianischen Fiskalpolitik zeigt, daß die Autoren ihr Augenmerk im ökonomischen Bereich primär auf allokative Ziele gerichtet haben. Weiterhin spielen die Interdependenzen zwischen okonomie und Politik eine zentrale Rolle. Makroökonomische Effekte werden zwar erwähnt, die Analyse geht aber über einige Ad-hoc-Hypothesen 1) und ein teilweises Aufgreifen monetaristischer Grundpositionen ${ }^{2)}$ nicht hinaus.

Aus diesem Grund scheint der SchluB gerechtfertigt, daB eine spezielle Kritik der keynesianischen Makroökonomik, deren Gültigkeit für die Situation der Unterbeschäftigung nicht bestritten wird ${ }^{3)}$, nicht Ziel der Arbeit von BW war.

Belegt wird diese Behauptung vor allem durch die Tatsache, daB die Kritik an der keynesianischen Fiskalpolitik als solcher lediglich auf von den Monetaristen bereits seit langem vorgetragene Thesen abhebt. Stattdessen wird die Aufmerksamkeit auf die von der makroökonomischen Literatur bisher weitgehend vernachlässigten Interdependenzen zwischen ökonomischem und politischem Sektor und auf die aus diesen Interdependenzen resultierenden allokativen Wirkungen fiskalpolitischer Maßnahmen gelenkt.

Die These, daß makroökonomische Oberlegungen für die Autoren eine untergeordnete Rolle spielen, wird noch erhärtet, wenn man sich eine von BUCHANAN an anderer Stelle gegebene Begründung für eine Budgetausgleichs- und Geldmengenregel vergegenwärtigt. In der zu-

1) Z.B. die empirisch nicht belegte Behauptung, Budgetdefizite führten zu Preisste igerungen.

2) So z.B. im 11. Kapitel von "Democracy in Deficit". Vgl. auch Buchanan/Wagner (1978), S. 635, wo die Hypothese der Stabilität des marktwirtschaftlichen Systems aufgestelit wird.

3) Vgl. Buchanan/Wagner (1977), S. $164 \mathrm{ff}$. 
sammen mit BRENNAN veröffentlichten Arbeit "The Power to Tax"1) werden die Budgetausgleichsrege ${ }^{2)}$ und die Geldmengenregel ${ }^{3}$ als Mittel gegen einen einnahmemaximierenden Leviathan-Staat dargestellt. Nicht makroökonomische Gründe erfordern aus dieser Sicht eine Geldmengenregel, sondern die Tatsache, daB diese Regel einer Einnahmeerhöhung des Leviathan-Staates im Wege eines Inflationsprozesses entgegenwirkt.

Während gegen das Aufgreifen monetaristischer Kritikpunkte gegen die keynesianische Fiskalpolitik wenig einzuwenden ist, muB die Vernachlässigung der Diskussion makroökonomischer Konsequenzen der eigenen Alternativlösung bemängelt werden. Die Vernachlässigung politischer Zusammenhänge in dem kritisierten Konzept der herrschenden Makroökonomik darf nicht zur Vernachlässigung makroökonomischer Effekte im Alternativkonzept der politischen okonomik führen.

Diese Kritik muB dem Vorschlag der Realisierung eines materiellen Budgetausgleichs nach Ablauf einer obergangsperiode, vor allem aber der für die Obergangsphase selbst formulierten 20 \%-Regel entgegengehalten werden. Diese Regel erscheint ohne konkrete Ausgesta1tung und makroökonomische Fundierung zu starr und ist daher in der vorgeschlagenen Form als Verfassungsregel abzulehnen. In einer Wirtschaft, deren Staatshaushalt der Konsolidierung bedarf, mag die Aussage "There is no costless cure for a maladjusted economy" 4 ) zwar zutreffen. Sie macht dennoch Oberlegungen, diese Kosten möglichst klein zu halten, nicht überflüssig. Erforderlich ist somit eine geeignete "Theorie des ubergangs" von einer durch die bisherigen institutionellen Regelungen bestimmten Wirtschaft zu einer nach neueren Erkenntnissen verbesserten Regelstruktur.

1) Vgl. Brennan/Buchanan (1980)

2) $\mathrm{Vgl}$. ebenda, S. 203

3) $\mathrm{Vgl}$. ebenda, S. $131 \mathrm{ff}$.

4) Vgl. Buchanan/Wagner (1977), S. 170 
In diesem Zusammenhang darf auch ein Ansatz, der kurzfristige Beschäftigungsprobleme als notwendigen Bestandteil der volkswirtschaftlichen. Entwicklung ansieht, die mit der Anpassung der Wirtschaft an eine veränderte Präferenz- und Technologiestruktur verbundenen Reibungsverluste nicht bagatellisieren.

Weiterhin muB die Vernachlässigung der mit der Steuerfinanzierung von Investitionsgütern verbundenen negativen Allokationseffekte kritisiert werden. Unter den von BW gesetzten Annahmen über den politischen Prozeß und die Wahrnehmungsasymmetrien der Wirtschaftssubjekte führt eine Defizitfinanzierung öffentlicher Konsumgüter zwar zu einer allokativ ineffizienten oberversorgung mit diesen Gütern. Unter denselben Annahmen bedingt jedoch eine Steuerfinanzierung öffentlicher Investitionsgüter eine Unterversorgung mit Gütern, deren Nutzungsabgabe nicht auf die Gegenwart begrenzt ist.

Die Vernachlässigung dieser Effekte ist vor allem deshalb überraschend, weil BUCHANAN in einer früheren Publikation selbst auf die Gefahr der Verzerrung von öffentlichen Investitionsentscheidungen bei Realisierung eines materiellen Budgetausgleichs hinweist. Indem er der "public debt illusion" auf der Einnahmenseite die "asset illusion" auf der Ausgabenseite gegenüberstellt, trägt er explizit der Tatsache Rechnung, daB die zukünftigen Nutzen von Investitionsgütern von den wählern genauso wenig wahrgenommen werden wie die mit Defiziten verbundenen zukünftigen Zins- und Tilgungsverbindlichkeiten ${ }^{1)}$. Er kommt demzufolge zu dem Ergebnis, daß eine Finanzverfassung die Staatsschuld durchaus als geeignetes Finanzierungsinstrument für öffentliche Investitionsgüter vorsehen $\operatorname{kann}{ }^{2}$ ).

1) "The debt illusion has its converse, however, and when this is also recognized, public debt again assumes a limited but legitimate place in the array of fiscal instruments. . . Buchanan (1967), S. 264. "In other words, for projects that involve benefits which accrue over time, there may exist some 'asset illusion'. The individual may not fully capitalize the future benefits that such outlays will yield. - Budgetary decisions will tend to be biased in favor of short-term and against long-term public projects." Ebenda, S. 265

2) Vgl. ebenda 
Die Tatsache, daB in der Schrift "Democracy in Deficit" lediglich mit der Annahme der "public debt illusion" gearbeitet und folglich ein materieller Budgetausgleich gefordert wird, läßt somit den SchluB zu, daB die mit der Staatsverschuldung verbundenen negativen Allokationseffekte in der Gegenwart verstärkt hervorgehoben werden sollten. Möglicherweise liegt der Argumentation bereits implizit die in späteren Arbeiten verwendete Annahme eines einnahmemaximierenden Leviathan-Staates zugrunde, den es nach Ansicht von BUCHANAN zu beschränken gilt ${ }^{1}$. Negative Effekte auf die Beschäftigung und auf die Bereitstellung öffentlicher Investitionsgüter werden bei der Verfolgung dieses Ziels offensichtlich bewußt in Kauf genommen.

Wie immer man zu der These eines einnahmemaximierenden LeviathanStaates stehen mag ${ }^{2}$, die Vernachlässigung negativer Effekte auf ein so bedeutendes Ziel wie die Vollbeschäftigung und auf die intertemporale Ressourcenallokation kann mit dieser These nicht gerechtfertigt werden ${ }^{3}$ ).

Dennoch ist der Grundgedanke der Arbeit von BW, Defizite künftig auf das zur Lösung einiger spezifischer Probleme notwendige Ausma $B$ zu begrenzen, als beachtlicher Impuls zu werten. Die in Abschnitt VII.4. angeregte Anderung der Finanzverfassung ist daher in erster Linie als Weiterentwicklung der von BW geforderten Verfassungsänderung zu sehen. So wirkt das Erfordernis einer Defizitfinanzierung sehr langfristiger Investitionsguter einer im Falle des materiellen Budgetausgleichs resultierenden Diskriminierung statlicher Investitionen entgegen. Die weiterhin vorgeschlagene Festsetzung ei-

1) Vgl. zu diesem Ansatz vor allem Brennan/Buchanan (1980) sowie Brennan/Buchanan (1979), (1978) und (1977).

2) Brennan/Buchanan räumen selbst ein, daß diese These einen theoretischen Grenzfall darstellt und in der Realität nur in der Tendenz zutrifft. Vgl. Brennan/ Buchanan (1980), S. $15 \mathrm{f}$.

3) Im übrigen läßt sich der Leviathan-Staat auch durch nichtfiskalische institutionelle Regeln beschränken. Vgl. West/Corke (1980), S. $401 \mathrm{f}$. 
ner kritischen Arbeitslosenquote, welche zu Beginn der Haushaltsperiode mit Zwei-Drittel-Mehrheit erfolgen soll, und bei deren oberschreitung ein statiiches deficit-spending erlaubt wird, vermeidet die mit einem materiellen Haushaltsausgleich in Rezessionsphasen verbundenen negativen Beschäftigungseffekte und bietet der Regierung zudem die Möglichkeit einer aktiven Beschäftigungspolitik.

Das Verdienst der Autoren liegt vor allem darin, den bisher von den meisten Makroökonomen vernachlässigten Zusammenhang zwischen ökonomischem und politischem Sektor sowie die Bedeutung von institutionellen Regeln für das Ergebnis ökonomischer Prozesse herausgearbeitet zu haben. Ihre Analyse macht deutlich, daß auch die Makroökonomie politisch-ökonomische Zusammenhänge beachten muß. Makroökonomische konzepte nützen wenig, wenn sie die herrschende Struktur politischer und ökonomischer Institutionen nicht beachten $^{1)}$. Die politisch-ökonomische Analyse keynesianischer Konzepte in demokratischen Staten liefert ein eindrucksvolles Beispiel für diese These.

1) $\mathrm{Vgl}$. in diesem Zusammenhang auch die auf dem politisch-ökonomischen Ansatz basierende "Theorie demokratischer Wirtschaftspolitik" von Frey (1978) und (1981). 
ANHANG

1. Zitate aus Interviews zur Unabhängigkeit des Federal Reserve System

Kommentar von Präsident Nixon zur Ernennung von Arthur Burns zum Vorsitzenden des Board of Governors:

"I hope that independently he will conclude that my views are the ones that should be followed."

Aus: The Economy: Arthur the Independent, in: Time Magazin (March 1, 1970), zitiert nach Borins (1972), S. 186

Antwort von Secretary Dillon, als er von einem Mitglied des Kongresses danach gefragt wurde, wie sich der Board-Vorsitzende im Falle einer Direktive seitens des Präsidenten verhalten würde:

"I think that under the present arrangement. . he would take that sort of advice very seriously, and it would deeply influence any decision he might reach as to his own position and as to his advice to his committee."

(Nach Weintraub (1978), S. 352)

Interview mit E. Ettin, Assistant Adviser im BOG. Es wurde im Jahre 1971 zu einer Zeit durchgeführt, in der die Regierung eine größere Wachstumsrate der Geldmenge befürwortete als die Mehrheit der FOMCMitglieder für ökonomisch vertretbar hielt:

"I asked what would happen if President $\mathrm{Nixon}$ were to phone the Governors to ask them to 'go along' with him by permitting a faster rate of growth of the money supply. They replied, 'Though there would be a small minority which would vote against it, the FOMC would go along'."

(Borins (1972), S. 196) 
2. Datenreihen zu Abschnitt v.2.2.2.1.3.1)

\begin{tabular}{|c|c|c|c|c|c|c|}
\hline \multicolumn{2}{|c|}{ Quartal } & $i^{D}$ & $\hat{\mathbf{p}}$ & $\hat{p}^{\star}$ & $A L Q$ & $A L Q^{*}$ \\
\hline 1969 & $\begin{array}{l}\text { I } \\
\text { I I } \\
\text { I I I } \\
\text { I V }\end{array}$ & $\begin{array}{l}3,0 \\
5,0 \\
6,0 \\
6,0\end{array}$ & $\begin{array}{l}2,2 \\
2,7 \\
2,7 \\
2,8\end{array}$ & $\begin{array}{l}2,0 \\
2,0 \\
2,0 \\
2,0\end{array}$ & $\begin{array}{l}1,0 \\
0,8 \\
0,7 \\
0,7\end{array}$ & $\begin{array}{l}1,0 \\
1,0 \\
1,0 \\
1,0\end{array}$ \\
\hline 1970 & $\begin{array}{l}\text { I } \\
\text { I I } \\
\text { I I I } \\
\text { I V }\end{array}$ & $\begin{array}{l}7,5 \\
7,5 \\
7,0 \\
6,0\end{array}$ & $\begin{array}{l}2,7 \\
3,2 \\
3,6 \\
3,8\end{array}$ & $\begin{array}{l}3,0 \\
3,0 \\
3,0 \\
3,0\end{array}$ & $\begin{array}{l}0,7 \\
0,6 \\
0,6 \\
0,7\end{array}$ & $\begin{array}{l}0,8 \\
0,8 \\
0,8 \\
0,8\end{array}$ \\
\hline 1971 & $\begin{array}{l}\text { I } \\
\text { I I } \\
\text { I I I } \\
\text { I V }\end{array}$ & $\begin{array}{l}6,0 \\
5,0 \\
5,0 \\
4,0\end{array}$ & $\begin{array}{l}4,6 \\
5,0 \\
5,7 \\
5,8\end{array}$ & $\begin{array}{l}3,0 \\
3,0 \\
3,0 \\
3,0\end{array}$ & $\begin{array}{l}0,7 \\
0,8 \\
0,9 \\
1,0\end{array}$ & $\begin{array}{l}0,8 \\
0,8 \\
0,8 \\
0,8\end{array}$ \\
\hline 1972 & $\begin{array}{l}\text { I } \\
\text { I I } \\
\text { I I I } \\
\text { I V }\end{array}$ & $\begin{array}{l}3,0 \\
3,0 \\
3,0 \\
4,5\end{array}$ & $\begin{array}{l}5,3 \\
6,5 \\
5,5 \\
6,3\end{array}$ & $\begin{array}{l}4,5 \\
4,5 \\
4,5 \\
4,5\end{array}$ & $\begin{array}{l}1,1 \\
1,1 \\
1,2 \\
1,2\end{array}$ & $\begin{array}{l}1,0 \\
1,0 \\
1,0 \\
1,0\end{array}$ \\
\hline 1973 & $\begin{array}{l}\text { I } \\
\text { I I } \\
\text { I I I } \\
\text { I V }\end{array}$ & $\begin{array}{l}5,0 \\
7,0 \\
7,0 \\
7,0\end{array}$ & $\begin{array}{l}6,5 \\
7,3 \\
6,9 \\
7,3\end{array}$ & $\begin{array}{l}5,8 \\
5,8 \\
5,8 \\
5,8\end{array}$ & $\begin{array}{l}1,0 \\
1,1 \\
1,2 \\
1,5\end{array}$ & $\begin{array}{l}1,0 \\
1,0 \\
1,0 \\
1,0\end{array}$ \\
\hline 1974 & $\begin{array}{l}\text { I } \\
\text { I I } \\
\text { I I I } \\
\text { I V }\end{array}$ & $\begin{array}{l}7,0 \\
7,0 \\
7,0 \\
6,0\end{array}$ & $\begin{array}{l}7,3 \\
7,0 \\
7,0 \\
6,5\end{array}$ & $\begin{array}{l}8,0 \\
8,0 \\
8,0 \\
8,0\end{array}$ & $\begin{array}{l}2,0 \\
2,3 \\
2,7 \\
3,4\end{array}$ & $\begin{array}{l}2,0 \\
2,0 \\
2,0 \\
2,0\end{array}$ \\
\hline 1975 & $\begin{array}{l}\text { I } \\
\text { I I } \\
\text { I I I } \\
\text { I V }\end{array}$ & $\begin{array}{l}5,0 \\
4,5 \\
3,5 \\
3,5\end{array}$ & $\begin{array}{l}6,0 \\
6,2 \\
6,0 \\
5,5\end{array}$ & $\begin{array}{l}6,0 \\
6,0 \\
6,0 \\
6,0\end{array}$ & $\begin{array}{l}3,9 \\
4,8 \\
5,2 \\
5,0\end{array}$ & $\begin{array}{l}3,0 \\
3,0 \\
3,0 \\
3,0\end{array}$ \\
\hline 1976 & $\begin{array}{l}\text { I } \\
\text { I I } \\
\text { I I I } \\
\text { I V }\end{array}$ & $\begin{array}{l}3,5 \\
3,5 \\
3,5 \\
3,5\end{array}$ & $\begin{array}{l}5,4 \\
4,9 \\
4,3 \\
3,9\end{array}$ & $\begin{array}{l}4,8 \\
4,8 \\
4,8 \\
4,8\end{array}$ & $\begin{array}{l}4,7 \\
4,7 \\
4,6 \\
4,5\end{array}$ & $\begin{array}{l}4,5 \\
4,5 \\
4,5 \\
4,5\end{array}$ \\
\hline 1977 & $\begin{array}{l}\text { I } \\
\text { I I } \\
\text { I I I } \\
\text { I V }\end{array}$ & $\begin{array}{l}3,5 \\
3,5 \\
3,5 \\
3,0\end{array}$ & $\begin{array}{l}3,7 \\
3,7 \\
3,8 \\
3,6\end{array}$ & $\begin{array}{l}4,0 \\
4,0 \\
4,0 \\
4,0\end{array}$ & $\begin{array}{l}4,3 \\
4,6 \\
4,7 \\
4,6\end{array}$ & $\begin{array}{l}4,0 \\
4,0 \\
4,0 \\
4,0\end{array}$ \\
\hline 1978 & $\begin{array}{l}\text { I } \\
\text { I I } \\
\text { I I I } \\
\text { I V }\end{array}$ & $\begin{array}{l}3,0 \\
3,0 \\
3,0 \\
3,0\end{array}$ & $\begin{array}{l}3,0 \\
2,8 \\
2,4 \\
2,5\end{array}$ & $\begin{array}{l}3,5 \\
3,5 \\
3,5 \\
3,5\end{array}$ & $\begin{array}{l}4,4 \\
4,5 \\
4,3 \\
4,2\end{array}$ & $\begin{array}{l}4,5 \\
4,5 \\
4,5 \\
4,5\end{array}$ \\
\hline 1979 & $\begin{array}{l}\text { I } \\
\text { I I } \\
\text { I I I } \\
\text { I V }\end{array}$ & $\begin{array}{l}4,0 \\
4,0 \\
5,0 \\
6,0\end{array}$ & $\begin{array}{l}2,9 \\
3,5 \\
4,7 \\
5,3\end{array}$ & $\begin{array}{l}3,0 \\
3,0 \\
3,0 \\
3,0\end{array}$ & $\begin{array}{l}4,1 \\
3,8 \\
3,7 \\
3,6\end{array}$ & $\begin{array}{l}4,0 \\
4,0 \\
4,0 \\
4,0\end{array}$ \\
\hline 1980 & I & $\begin{array}{l}7,0 \\
7,5\end{array}$ & $\begin{array}{l}5,5 \\
5,9\end{array}$ & $\begin{array}{l}4,5 \\
4,5\end{array}$ & $\begin{array}{l}3,6 \\
3,6\end{array}$ & $\begin{array}{l}3,8 \\
3,8\end{array}$ \\
\hline
\end{tabular}

1) In den Jahren, in denen der Jahreswirtschaftsbericht einen Bereich für die Zielwerte vorsah, wurde für $z^{\star}$ das arithmetische Mittel der Zielbereiche verwendet (Zahlen mit zwei Stellen hinter dem Komma wurden aufgerundet). Im Jahre 1974 wurde für $\hat{p}^{\star}$ der untere Zielwert von $8 \%$ berücksichtigt. 
3. Eine "Reaktionsfunktion" der Zentralbank

Es wird unterstellt, da $B$ die Geldmengenänderung der laufenden Periode vor allem von der Sozialproduktsänderung derselben Periode und von der Geldmengenentwicklung in der Vergangenheit abhängt. Weiterhin wird wie im Falle der Diskontpolitik die Abweichung der Preissteigerungsrate und der Arbeitslosenquote von ihren wunschwerten berücksichtigt. Zum Test der Geldmengenwirkung von Budgetdefiziten wird außerdem das Budgetdefizit der laufenden Periode ${ }^{1)}$ in die Modellgleichung aufgenommen. SchlieBlich enthält die Modellgleichung die Anderung der Emissionsrendite für festverzinsliche Wertpapiere $\Delta r$, was eine Uberprüfung der These gestattet, die Bundesbank betreibe eine Politik der Zinsstabilisierung ${ }^{2}$.

Getestet wurde die Modellgleichung

$$
\Delta M_{t}=a+b \cdot \Delta M_{t-2}+c \cdot \Delta Y_{t}+d \cdot D E F_{t}
$$

$$
\begin{aligned}
& +e \cdot v_{t-2} \cdot\left(\hat{p}-\hat{p}^{\star}\right)_{t-2} \\
& +f \cdot W_{t-2} \cdot\left(A L Q-A L Q^{\star}\right)_{t-2}+g \cdot \Delta r_{t-\tau}+\alpha_{t} \cdot
\end{aligned}
$$

1) Weitere Schätzungen wurden mit dem Budgetdefizit der Vorperiode durchgeführt, ohne daß sich an den grundsätzlichen Ergebnissen etwas änderte.

2) Die Wahl dieses Zinssatzes ist sicherlich in gewisser Weise willkürlich. Da private Unternehmen zur Finanzierung ihrer Investitionen zumindest teilweise auf die Emission von Wertpapieren angewiesen sind, könnte die Emissionsrendite festverzinslicher Wertpapiere jedoch ein Indikator für potentielle zinsbedingte Crowding-out-Effekte sein.

3) Der Variablen $\Delta r$ wurde der Zeitindex $t-\tau$ zugeordnet, da die Gleichung in drei Varianten mit $\tau=0,1,2$ getestet wurde. W und $V$ sind Dummy-Variable, die immer dann, wenn das jeweilige Ziel stärker gefährdet war, den Wert 1, andernfalls den Wert 0 annehmen. 


\begin{tabular}{|c|c|c|c|c|c|c|c|c|c|c|}
\hline $\begin{array}{l}\text { Gleichungs- } \\
\text { nummer }\end{array}$ & $\Delta M_{t-2}$ & $\Delta Y_{t}$ & $D_{t}$ & $V \cdot\left(\hat{p}-\hat{p}^{\star}\right)_{t-2}$ & $W \cdot\left(A L Q-A L Q^{*}\right)_{t-2}$ & $\Delta r_{t-\tau}$ & $\tau$ & $\begin{array}{l}\text { Absolut- } \\
\text { glied }\end{array}$ & $\begin{array}{l}\text { Test- } \\
\text { statis } \\
\mathrm{R}^{2}\end{array}$ & $\begin{array}{l}\text { iken } \\
\text { FG }\end{array}$ \\
\hline$(v .22 .0)$ & $\begin{array}{l}0,4708 \star \star \\
\quad(12,765)\end{array}$ & $\begin{array}{l}0,6098 * \star \\
(25,590)\end{array}$ & - & - & - & - & - & 0,8897 & 0,653 & 26 \\
\hline$(v .22 .1)$ & $\begin{array}{l}0,4940 \star \star \\
(13,816)\end{array}$ & $\begin{array}{l}0,6243 \star \star \\
(25,886)\end{array}$ & $\begin{array}{l}0,1732 \\
\quad(0,160)\end{array}$ & $\begin{array}{r}-0,8265 \\
(0,039)\end{array}$ & $\begin{array}{r}-0,5868 \\
(0,062)\end{array}$ & $\begin{array}{r}-4,7880 \\
(3,492)\end{array}$ & 0 & 0,2505 & 0,717 & 22 \\
\hline$(v .22 .2)$ & $\begin{array}{l}0,4221 * \star \\
(9,069)\end{array}$ & $\begin{array}{l}0,6292 \star \star \\
(23,681)\end{array}$ & $\begin{array}{l}0,2097 \\
(0,318)\end{array}$ & $\begin{array}{r}-3,4942 \\
(0,858)\end{array}$ & - & $\begin{array}{r}-2,5923 \\
(1,004)\end{array}$ & 1 & 0,2321 & 0,685 & 23 \\
\hline (学.22.3) & $\begin{array}{l}0,4605^{\star \star} \\
(10,973)\end{array}$ & $\begin{array}{l}0,6084 * \star \\
(22,107)\end{array}$ & $\begin{array}{l}0,2678 \\
\quad(0,326)\end{array}$ & $\begin{aligned}-3, & 5979 \\
& (0,755)\end{aligned}$ & $\begin{array}{l}1,0889 \\
(0,201)\end{array}$ & $\begin{array}{l}2,4111 \\
(0,786)\end{array}$ & 2 & $-0,5824$ & 0,683 & 22 \\
\hline
\end{tabular}

1. Beihefte zu den Monatsberichten der Deutschen Bundesbank, Reihe 2, Wertpapierstatistik (verschiedene Ausgaben). Die Abweichungen wirtschaftspolitischer Ziele von ihren Wunschwerten wurden wie im Falle der Diskontpolitik auf der Basis saisonbereinigter Werte berechnet. Datenquelle: Statistische Beihefte zu den Monatsberichten der Deutschen Bundesbank, Reihe 4, Saisonbere inigte Wirtschaftszahlen. Vgl. zur Begründung S. 139, FuBnote 2. 


\section{LITERATUR}

Aaron, H., McGuire, $M$.

Acheson, K., Chant, J.F.

Amacher, R.C., Tollison, R.D.

Amacher, R.C., Tollison, R.D., Willet, Th.D.

Arcelsus, F., Meltzer, A.H.

Arcelsus, F., Meltzer, A.H.

Bach, G.L.

Bamberg, G., Schittko, U.K.

Barr, J., Davis, 0.A.

Barro, R.J.

Barro, R.J.

Barro, R.J.

Bartlett, R.
(1970) Public Goods and Income Distribution. Econometrica 38, $907-920$

(1973) Bureaucratic Theory and the Choice of Central Bank Goals. The Case of the Bank of Canada. Journal of Money, Credit and Banking $5,637-655$

(1974) Fiscal Preference and Balanced Budget Fiscal Policy. Public Choice 19, 107 - 109

(1975) A Budget Size in a Democracy: A Review of Arguments. Public Finance Quarterly 3,99-121

(1975) The Effect of Aggregate Economic Variables on Congressional Elections. American Political Science Review 69, 1232 - 1239

(1975a) Aggregate Economic Variables and Votes for Congress: A Rejoinder. American Political Science Review 69, 1266 - 1269

(1962) Federal Reserve Organization and Policy-making, in: Smith/Teigen (1974), 271 - 276; zuerst erschienen in: Harvard Business Review 40, January - February, 81 - 91

(1979) Einführung in die Okonometrie. Stuttgart u.a.0.

(1966) An Elementary Political and Economic Theory of the Expenditures of Local Governments. Southern Economic Journal, $149-165$

(1977) Unanticipated Money Growth and Unemployment in the United States. American Economic Review 62, $101-115$

(1978) Comment from an Unreconstructed Ricardian. Journal of Monetary Economics 4 , $569-581$

(1978a) Unanticipated Money, Output and the Price Level in the United States. Journal of Political Economy $86,549-580$

(1973) Economic Foundations of Political Power. New York 
Basler, H. -P.

Basler, H. - P.

Bastable, C.F.

Baumol, W.J.,

Blinder, A.S.

Beetz, M.

Bergstrom, Th.C.,

Goodman, R.P.

Bernholz, P.

Bernholz, P.

Besters, H.

Black, D.

Blackaby, F.T.

Blankart, C.B.

Blondel, J.

Bloom, H.S.,

Price, H.D.
(1978) Die wirtschaftspolitischen Zielpräferenzen der Deutschen Bundesbank. Eine empirische Analyse des Zentralbankverhaltens für die Zeit von 1958 bis 1974. Kredit und Kapital 11, $84-108$

(1979) Wirtschaftspolitische Zielpräferenzen und theoretische Orientierung in der Geldpolitik der Bundesrepublik Deutschland. Tüb ingen

(1903) Public Finance. 3rd Edition. London

(1979) Economics. Principles and Policy. New York u.a.o.

(1978) Sozialökonomische Analyse des Zusammenhangs von WahlenprozeB und Konjunktur. Dissertation Köln

(1973) Private Demands for Public Goods. American Economic Review 63, $280-296$

(1975) Grundlagen der Politischen Okonomie. 2. Band. Tübingen

(1979) Grundlagen der Politischen Okonomie. 3. Band. Tübingen

(1980) Wie kam es zur hohen Staatsverschuldung? Wirtschaftsdienst $60,419-424$

(1948) On the Rationale of Group Decision Making. Journal of Political Economy 56 , $23-34$

(1979) The Economics and Politics of Demand Management, in: Cook/Jackson (1979), 185 197

(1977) Neuere Ansätze zur Erklärung des Wachstums der Staatsausgaben. Ein interpretierender Oberblick. Hamburger Jahrbuch für Wirtschafts- und Gesellschaftspolitik 22 , $73-91$

(1963) Voters, Parties and Leaders. The Social Fabric of British Politics. Harmondsworth

(1975) Voter Response to Short-Run Economic Conditions: The Asymmetric Effect of Prosperity and Recession. American Political

Science Review 69, $1240-1254$ 
von Böhm-Bawerk, E.

Borcherding, T.E. (Ed.)

Borcherding, T.E.

Borcherding, T.E., Deacon, R.T.

Brainard, W.

Braun, $K$.

Brennan, G.,

Buchanan, J.M.

Brennan, G.,

Buchanan, J.M.

Brennan, G., Buchanan, J.M.

Brennan, G., Buchanan, J.M.

Brunner, $K$.

Brunner, $K$.
(1921) Positive Theorie des Kapitals. 1. Band, 4. Auflage. Jena

(1977) Budgets and Bureaucrats: The Sources of Government Growth. Durham

(1977a) The Sources of Growth of Public

Expenditures in the United States, 1902 -

1970 , in: Borcherding (1977), $45-70$

(1972) The Demand for the Services of NonFederal Governments. American Economic Review $62,891-901$

(1967) Uncertainty and Effectiveness of Policy. American Economic Review, Papers and Proceedings $57,411-425$

(1969) Die Stellung der Notenbank als währungspolitische Instanz. Das Verhältnis zwischen Regierung und Notenbank in der Bundesrepublik Deutschland und in den Vereinigten Staaten von Amerika. Dissertation Tübingen

(1977) Towards a Tax Constitution for Leviathan. Journal of Public Economics 8, 255 273

(1978) Tax Instruments as Constraints on the Disposition of Public Revenues. Journal of Public Economics 9, $301-318$

(1979) The Logic of Tax Limits: Alternative Constraints on the Power to Tax. National Tax Journal $32,11-32$

(1980) The Power to Tax. Analytical Foundations of a Fiscal Constitutuion. Cambridge u.a.o.

(1970) Eine Neuformulierung der Quantitätstheorie des Geldes. Die Theorie der relativen Preise, des Geldes, des outputs und der Beschäftigung. Kredit und Kapital 3, $1-30$

(1970a) Die "Monetaristische Revolution" der Geldtheorie, in: Kalmbach (1973), 70 103; zuerst erschienen als: The "Monetarist Revolution" in Monetary Theory. Weltwirtschaftliches Archiv 105/II (1970), 1 - 30. Die Seitenangaben beziehen sich auf die deutsche Quelle. 
Brunner, $K$.
Brunner, $K$.

Brunner, $K$.

Brunner, K., Meltzer, A.H.

Buchanan, J.M.

Buchanan, J.M.

Buchanan, J.M.

Buchanan, J.M.

Buchanan, J.M.

Buchanan, J.M.

Buchanan, J.M.
(1975) Comment. Journal of Law and Economics $28,837-858$

(1976) Issues of Post-Keynesian Monetary Analysis. A Contribution to the Discussion Opened by Professor Mayer. Kredit und Kapital $9,24-55$

(1978) Reflections on the Political Economy of Government. The Persistent Growth of Government. Schweizerische Zeitschrift für Volkswirtschaft und Statistik 114,649-680

(1976) An Aggregative Theory for a Closed Economy, in: J.L. Stein (Hrsg.), Monetarism. Amsterdam u.a.0., $69-103$

(1954) Individual Choice in Voting and the Market, in: J.M. Buchanan (1960), Fiscal Theory and Political Economy, Selected Essays, Chapel Hill, 90-104; zuerst erschienen in: Journal of Political Economy 62 , $334-343$

(1958) Public Principles of Public Debt. Homewood, I11.

(1962) Easy Budgets and Tight Money. Lloyds Bank Review 64, 17 - 30; wiederabgedruckt in: J.M. Buchanan, R.D. Tollison (Eds.), Theory of Public Choice. Political Applications of Economics (1972), Ann Arbor, 62 75

(1964) Fiscal Institutions and Efficiency in Collective Outlay. American Economic Review, Papers and Proceedings $54,227-235$

(1964a) Concerning Future Generations, in: Ferguson (1964), $55-63$

(1964b) Public Debt, Cost Theory and the Fiscal Illusion, in: Ferguson (1964), 150 163

(1967) Public Finance in Democratic Process. Fiscal Institutions and Individual Choice. Chapel Hill 
Buchanan, J.M.

Buchanan, J.M.

Buchanan, J.M.

Buchanan, J.M.

Buchanan, J.M., Burton, J., Wagner, R.E.

Buchanan, J.M., Burton, J., Wagner, R.E.

Buchanan, J.M., Wagner, R.E.

Buchanan, J.M., Wagner, R.E.

Buchanan, J.M., Wagner, R.E.

Buchanan, J.M., Wagner, R.E.

Buchanan, J.M., Wagner, R.E.

Buchanan, J.M., Wagner, R.E.

Buchanan, J.M., Wagner, R.E. (1967a) Fiscal Policy and Fiscal Preference. Public Choice 2, Spring, 1 - 10; wiederabgedruckt in: J.M. Buchanan, R.D. Tollison (Eds.), Theory of Public Choice. Political Applications of Economics (1972), Ann Arbor, 76 - 84. Die Seitenangaben beziehen sich auf die neuere Quelle.

(1975) The Limits of Liberty. Between Anarchy and Leviathan. Chicago

(1975a) A Contractarian Paradigm for Applying Economic Theory. American Economic Review, Papers and Proceedings 65, $225-230$

(1977) Why Does Government Grow? in: Borcherding (1977), $3-18$

(1978) The Consequences of Mr. Keynes. London

(1978a) Constitutional Options for Fiscal Control, in: Buchanan/Burton/Wagner (1978), $77-86$

(1967) Public Debt in a Democratic Society. Washington D.C.

(1977) Democracy in Deficit. The Political Legacy of Lord Keynes. New York u.a.0.

(1978) Dialogues Concerning Fiscal Religion. Journal of Monetary Economics 4, $627-636$

(1978a) Fiscal Responsibility in Constitutional Democracy. Edited by G. Tullock. Leiden u.a.o.

(1978b) Contemporary Democracy and the Proposal for Fiscal Control: Initial Thoughts About and Final Reactions to the Conference, in: Buchanan/Wagner (1978a), $1-8$

(1978c) The Political Biases of Keynesian Economics, in: Buchanan/Wagner (1978a), 79 100

(1978d) Democracy and Keynesian Constitutions: Political Biases and Economic Consequences, in: Buchanan/Burton/Wagner (1978), $11-27$ 
Bundesministerium der Finanzen

Burton, J.

Caesar, R.

Cansier, D.

Carlson, K.,

Spencer, R.W.

Casse1, D.

Cavaco-Silva, A.A.

Chant, J.F.,

Acheson, $K$.

Commission on

Money and Credit

\section{Cook, S.T.,}

Jackson, P.M.

Cowart, A.T.

Crain, M.,

Ekelund, R.B.

Culbertson, J.M.
(1978) Die Maßnahmen zur Stärkung der Nachfrage und zur Verbesserung des Wirtschaftswachstums. Reihe Bürger-Informationen. Bonn

(1978) Keynes's Legacy to Great Britain:

"Folly in a Great Kingdom", in: Buchanan/

Burton/Wagner (1978), $29-75$

(1979) Der Handlungsspielraum der Notenbanken - Theoretische Analyse und internationaler Vergleich -. Habilitation Köln

(1977) Einwände gegen die Wirksamkeit der Fiskalpolitik. Kredit und Kapital 10, 4564

(1975) Crowding Out and its Critics. Federal Reserve Bank of St. Louis Review 57, December, $2-17$

(1980) Wachsende Staatsverschuldung - Wohl tat oder Plage? Entwicklungstendenzen und volkswirtschaftliche Auswirkungen der öffentlichen Verschuldung in der Bundesrepublik Deutschland. List-Forum 10/5 (1979/80), $265-283$

(1977) Economic Effects of Public Debt. London

(1972) The Choice of Monetary Instruments and the Theory of Bureaucracy. Public Choice 12, Spring, $13-33$

(1961) Money and Credit. Their Influence on Jobs, Prices and Growth. The Report of the Commission on Money and Credit. Englewood Cliffs

(1979) Current Issues in Fiscal Policy. oxford

(1978) The Economic Policies of European Governments, Part I: Monetary Policy. British Journal of Political Science 8, 285 311

(1978) Deficits and Democracy. Southern Economic Journal $44(1977 / 78), 813-828$

(1972) Money and Banking. New York u.a.o. 
Dalton, H.

Dernburg, T.F., McDougal1, D.M.

Deutsche Bundesbank

Deutsches Institut für Wirtschaftsforschung

Dickertmann, D.

Dieckheuer, G.

Dieckheuer, G.

Dieckheuer, G.

Dieckheuer, G.

Dinke1, R.
(1954) Principles of Public Finance. 4th Edition. London

(1974) Lehrbuch der Makroökonomischen Theorie. Stuttgart

(1980) Die währungspolitischen Institutionen und Instrumente in der Bundesrepublik Deutschland. 3. überarbeitete Auflage des Kapitels "Bundesrepublik Deutschland" der von der EWG herausgegebenen Studie "Die Währungspolitik in den Ländern der Europäischen Wirtschaftsgemeinschaft - Institutionen und Instrumente -". Sonderdruck der Deutschen Bundesbank 1. Frankfurt/M.

(1980) Lange Reihen zur vierteljährlichen volkswirtschaftlichen Gesamtrechnung für die Bundesrepublik Deutschland. Ausgewählte Ursprungswerte und saisonbereinigte Daten von I/1960 bis I/1980. Berlin

(1975) Die Autonomie der Bundesbank unter dem EinfluB geldpolitischer Entwicklungen. Wirtschaftspolitische Chronik $24 / 1,23-45$

(1977) Möglichkeiten und Risiken einer kreditfinanzierten Stabilisierungspolitik. Wirtschaftsdienst $57,553-561$

(1978) Staatsverschuldung und wirtschaftliche Stabilisierung. Baden-Baden

(1979) Zu den Wirkungen einer öffentlichen Kreditaufnahme auf die funktionelle und die personel le Einkommensverteilung. Finanzarchiv N.F. $37,1-25$

(1980) Der Crowding-out-Effekt. Zum gegenwärtigen Stand von Theorie und Empirie. Deutsches Institut für Wirtschaftsforschung. Vierteljahreshefte zur Wirtschaftsforschung $47,126-147$

(1977) Der Zusammenhang zwischen der ökonomischen und politischen Entwicklung in einer Demokratie. Eine Untersuchung mit Hilfe der ökonomischen Theorie der Politik. Berlin 
Dinke1, R.

Dornbusch, R.,

Fischer, $S$.

Downs, $A$.

Downs, A.

Duwendag, D.

Duwendag, D.,

Ketterer, K.-H.,

Kösters, W.,

Poh1, R.,

Simmert, D.B.

Ehrenberg, $\mathrm{H}$.

Ehrlicher, w.

Ehrlicher, w.

Eisner, R.

Erb, R.D. (Ed.)

Erb, R.D.

Fair, R.C.

Fair, R.C.
(1978) Der Zusammenhang zwischen Regierungs popularität und ökonomischen Variablen. Einige theoretische und empirische Oberlegungen, in: E. Helmstädter (Hrsg.), Neuere Entwicklungen in den Wirtschaftswissenschaften, Schriften des Vereins für Socialpolitik N.F. 98 , Berlin, $543-561$

(1978) Macroeconomics. New York u.a.0.

(1957) An Economic Theory of Democracy. New York

(1967) Inside Bureaucracy. Boston

(1978) Staatsverschuldung und Zentralbankgeldmengensteuerung (I) und (II). Das Wirtschaftsstudium $7,132-138$ und $184-189$

(1977) Geldtheorie und Geldpolitik. 2. Auf lage. Köln

(1976) Zwischen Marx und Markt, vom Autor für die Taschenbuchausgabe durchgesehene und ergänzte Fassung. München

(1975) Das strukturelle Defizit. Wirtschafts dienst $55,449-453$

(1979) Grenzen der öffentlichen Verschuldung. Wirtschaftsdienst $59,393-400$

(1977) Capital Shortage: Myth and Reality. American Economic Review, Papers and Proceedings $67,110-115$

(1978) Federal Reserve Policies and Public Disclosure. Washington

(1978a) Introduction and Summary, in: Erb $(1978), 1-12$

(1975) On Controlling the Economy to Win Elections. Cowles Discussion Paper 397

(1978) The Effect of Economic Events on Votes for President. The Review of Economics and Statistics $60,159-173$ 
Feldste in, M.

Ferguson, J.M. (Ed.)

Ferguson, J.M.

Fisher, I.

Flowers, M.R.

Folkers, C.

Folkers, C.

Folkers, C.

Francke, H. $-\mathrm{H}$.

Frey, B.S.

Frey, B.S.

Frey, B.S.

Frey, B.S.

Frey, B.S.

Frey, B.S.
(1980) Fiscal Policies, Inflation, and Capital Formation. American Economic Review $70,636-650$

(1964) Public Debt and Future Generations. Chapel Hill

(1964a) Temporal Utility and Fiscal Burden, in: Ferguson $(1964), 219-228$

(1932) Die Zinstheorie. Jena

(1977) Revenue Structure, Fiscal Illusion, and Budgetary Choice: Comment. Public Choice 29, Spring, $127-130$

(1979) Staatswirtschaftliche Quoten und Beziehungszahlen (I) und (II). Das Wirtschaftsstudium $8,405-409$ und $457-463$

(1980) Vermögen I: Struktur und Verteilung. Handwörterbuch der Wirtschaftswissenschaft, Band 8 , Stuttgart u.a.0., $265-282$

(1981) Vermögensverteilung und staatliche Aktivität. Zur Theorie distributiver Prozesse im Interventionsstaat. Frankfurt/M. u.a.0.

(1979) Ober- und Untergrenzen der öffentlichen Verschuldung. Ein kritischer Kommentar zu Ausführungen von Wolfgang Stützel. Kredit und Kapital 12,363-376

(1975) Eine Einschätzung der Neuen Politischen okonomie der 70 er Jahre. Zeitschrift für die gesamte Staatswissenschaft 31,697. 718

(1977) Moderne Politische Okonomie. München - Zürich

(1978) Eine Theorie demokratischer Wirtschaftspolitik. Kyklos 31,208-234

(1978a) Der EinfluB der Wirtschaftslage auf Wählerentscheidungen. Wirtschaftswissenschaftliches Studium 7, $553-556$

(1978b) Politico-Economic Models and Cycles. Journal of Public Economics 9, $203-220$

(1979) Economic Policy by Constitutional Contract. Kyklos 32,307-319 
Frey, B.S.

Frey, B.S.

Frey, B.S., Garbers, $H$.

Frey, B.S., Garbers, $H$.

Frey, B.S., Ramser, H. -J.

Frey, B.S., Schneider, F.

Frey, B.S., Schneider, F.

Frey, B.S., Schneider, F.

Frey, B.S., Schneider, F.

Freyer, $W$.

Freyer, W., Widma ier, H.P. (1979a) Politisch-ökonomische Modelle. Wirtschaftswissenschaftliches Studium 8 , $5-8$

(1981) Theorie demokratischer Wirtschafts politik. München

(1971) Politico Econometrics - On Estimation in Political Economy. Political Studies $19,316-320$

(1972) Der Einfluß wirtschaftlicher Variabler auf die Popularität der Regierung eine empirische Analyse. Jahrbücher für Nationalökonomie und Statistik 186 (1971/72), $281-295$

(1976) The Political Business Cycle: A Comment. The Review of Economic Studies 43, $553-555$

(1976) An Econometric Model with an Endogenous Government Sector. Diskussionsbeitrag des Fachbereichs Wirtschaftswissenschaften und Statistik, Universität Konstanz

(1978) An Empirical Study of Politico-Economic Interaction in the United States. The Review of Economics and Statistics $60 / 2$, $174-183$

(1978a) A Politico-Economic Model of the United Kingdom. The Economic Journal 88 , $243-253$

(1978b) Central Bank Behavior: A Positive Empirical Analysis. Diskussionsbeiträge des Instituts für empirische Wirtschaftsforschung 7803, Universität Zürich. Eine revidierte Version ist inzwischen erschienen in: Journal of Monetary Economics 7 (1981), $291-315$

(1979) Der wahlzyklische Verlauf von Arbeitslosenquote und Inflation in der Bundesrepublik Deutschland. Einige Thesen und deren empirische oberprüfung, in:

Hillinger/Holler (1979), $150-171$

(1979) Zur Kritik wahlpolitischer Erklärungsversuche der Wirtschaftspolitik. Jahrbuch für Sozialwissenschaft 30, $162-176$ 
Friedlaender, A.F.

Friedman, M.

Friedman, M.

Froyen, R.T.

Gäfgen, G.

Gäfgen, G.

Gärtner, M.

Gandenberger, 0 .

Gandenberger, 0 .

Gandenberger, 0 .

Gandenberger, 0 .
(1973) Macro Policy Goals in the Postwar Period: A Study in Revealed Preference. The Quarterly Journal of Economics $87,25-43$

(1956) The Quantity Theory of Money - A Restatement, in: M. Friedman (Ed.), Studies in the Quantity Theory of Money, Chicago

(1959) A Program for Monetary Stability. New York

(1974) A Test of the Endogenity of Monetary Policy. Journal of Econometrics 2, 175 188

(1977) Neo-Institutionalismus - ein Weg zur Analyse und Reform zeitgenössischer Gesellschaften? Betrachtungen am Beispiel von J.K. Galbraith. Hamburger Jahrbuch für Wirtschafts - und Gesellschaftspolitik 22, 151 177

(1979) Zusammenfassung der Diskussion, in: C.C. von Weizsäcker (Hrsg.), Staat und Wirtschaft, Schriften des Vereins für Socialpolitik, Gesellschaft für Wirtschafts- und Sozialwissenschaften N.F. $102,498-502$

(1980) Zur Endogenisierung der Wirtschaftspolitik durch die Neue Politische okonomie. Bemerkungen zu einem Beitrag von W. Freyer und H.P. Widmaier. Jahrbuch für Sozialwissenschaft $31,253-260$

(1970) Ist es möglich, durch die Aufnahme von öffentlichem Kredit die Last einer öffentlichen Ausgabe in die Zukunft zu verschieben? Jahrbuch für Sozialwissenschaft $21,87-98$

(1970a) Offentlicher Kredit und Einkommensverteilung. Finanzarchiv $29,1-16$

(1971) Zur Rationalität der öffentlichen Kreditaufnahme. Verhaltenshypothesen und normative Konsequenzen. Finanzarchiv 30 , $369-391$

(1972) Intertemporale Verteilungswirkungen der Staatsverschuldung, in: H. Haller, W. Albers (Hrsg.), Probleme der Statsverschuldung, Berlin, $189-214$ 
Gandenberger, 0 .

Gandenberger, 0 .

Gandenberger, 0 .

Geisler, R.P.

Goetz, C.J.

Goodhart, C.A.E.,

Bhansali, R.J.

Goodman, S.,

Kramer, G.H.

Gordon, D.F.

Gordon, D.F.

Gordon, R.J.

Greene, K.V.

Hahn, 0 .

Hamburger, M.J.,

Zwick, B.

Hansmeyer, K.H.
(1974) Die Wirkung des öffentlichen Kredits auf die Einkommensverteilung. Das Wirtschaftsstudium $3,383-387$

(1980) Theorie der öffentlichen Verschuldung. Handbuch der Finanzwissenschaft, 3. Auflage, Band III, Tübingen, $3-49$

(1980a) Offentliche Verschuldung II. Theoretische Grundlagen. Handwörterbuch der Wirtschaftswissenschaft, Band 5, Stuttgart u.a.0., $480-504$

(1953) Notenbankverfassung und Notenbankentwicklung in USA und Westdeutschland. Berlin

(1977) Fiscal Illusion in State and Local Finance, in: Borcherding (1977), 176 - 187

(1970) Political Economy. Political Studies $18,43-106$

(1975) Comment on Arcelsus and Meltzer. The Effect of Aggregate Economic Conditions on Congressional Elections. The American Political Science Review 69, $1255-1265$

(1978) Debts, Keynes and Our Present Discontents. Journal of Monetary Economics 4, $583-589$

(1978a) Comments, in: Buchanan/Wagner $(1978 a), 24-28$

(1978) Macroeconomics. Boston - Toronto

(1973) An Empirical Test of the Wagner Debt Illusion Hypothesis. Issues in Urban Public Finance, Saarbrücken, $208-225$

(1976) Praktiker . . / . . oder Politiker. Zeitschrift für das gesamte Kreditwesen $29,527-528$

(1981) Deficits, Money and Inflation. Journal of Monetary Economics 7, $141-150$

(1968) Wandlungen im Handlungsspielraum der Notenbank? in: C.A. Andreae, K.H. Hansmeyer, G. Scherhorn (Hrsg.), Geldtheorie und Geldpolitik. Güter Schmölders zum 65. Geburtstag, Berlin, $155-166$ 


\begin{abstract}
Harrod, R.
Hauser, H.J.

Havrilesky, T.

Hayek, F.A.

He in, S.E.

Hendershott, P.H.

Hillinger, C., Holler, M.J. (Hrsg.)

Hirshleifer, J. Hobbes, T.

Horvitz, P.M.

Huppertz, P. $-H$.

Jarchow, H.J.

Johnson, H.G.

(1951) The Life of John Maynard Keynes. London

(1979) Verteilungswirkungen der Staatsverschuldung. Eine kreislauftheoretische Inzidenzbetrachtung. Frankfurt/M. U.a.0.

(1967) A Test of Monetary Policy Action. Journal of Political Economy 75, 299 - 304

(1935) Prices and Production. 2nd Edition. London

(1981) Deficits and Inflation. Federel Reserve Bank of St. Louis Review 63, March, $3-10$

(1976) A Tax Cut in a Multiple Security Mode1: Crowding-0ut, Pulling-In and the Term Structure of Interest Rates. Journal of Finance $31,1185-1199$

(1979) Okonomische Theorie der Politik. München

(1974) Kapitaltheorie. Köln

(1651) Leviathan oder Stoff, Form und Gewalt eines bürgerlichen und kirchlichen Staates, herausgegeben und eingeleitet von J. Fetscher (1966), Neuwied - Berlin

(1969) Monetary Policy and the Financial System. 2nd Edition. Englewood Cliffs

(1977) Gewaltenteilung und antizyklische Finanzpolitik. Baden-Baden

(1974) Theorie und Politik des Geldes II. Geldmarkt und geldpolitische Instrumente. Göttingen

(1964) Should There Be an Independent Monetary Authority? in: Smith/Teigen (1974); zuerst erschienen in: The Federal Reserve System after Fifty Years. Hearings before the Subcommittees on Domestic Finance, Committee on Banking and Currency, House of Representatives, 88 th Congress, 2 nd Session (1964), Washington
\end{abstract}


Johnson, H.G.

Johnson, H.G.

Johnson, H.G.

Johnston, J.

Jonung, L., Wadensjö, E.

Ka a se, M.

Kaiser, R.H.

Kalmbach, P. (Hrsg.)

Kane, E.J.

Kaufmann, G.G.

Kent, R.P.

Kirchgäßner, G .

Kirchgäßner, G.
(1971) Die keynesianische Revolution und die monetaristische Konterrevolution, in: Kalmbach (1973), 196 - 216; zuerst erschienen als: The Keynesian Revolution and the Monetarist Counter-Revolution. American Economic Review, Papers and Proceedings 61, 1 14

(1975) Living with Inflation. The Banker $125,863-864$

(1975a) Inflation. Theorie und Politik. München

(1972) Econometric Methods. 2nd Edition. International Student Edition. Tokyo u.a.o.

(1979) The Effect of Unemployment, Inflation and Real Income Growth on Government Popularity in Sweden. The Scandinavian Journal of Economics $81 / 2,343-353$

(1973) Die Bundestagswahl 1972: Probleme und Analysen. Politische Vierteljahresschrift 14 , $145-190$

(1980) Bundesbankautonomie - Möglichkeiten und Grenzen einer unabhängigen Politik. Frankfurt/M.

(1973) Der neue Monetarismus. München

(1980) Politics and Fed Policy-making. The More Things Change the More Remains the Same. Journal of Monetary Economics 6, 199 - 211

(1973) Money, the Financial System and the Economy. Chicago u.a.o.

(1966) Money and Banking. 5th Edition. New York u.a.0.

(1974) Okonometrische Untersuchungen des Einflusses der Wirtschaftslage auf die Popularität der Parteien. Schweizerische Zeitschrift für Volkswirtschaft und statistik $110,409-445$

(1976) Rationales Wählerverhalten und optimales Regierungsverhalten. Dissertation Konstanz 
Kirchgäßner, G .

Kirchgäßner, G., Schneider, F.

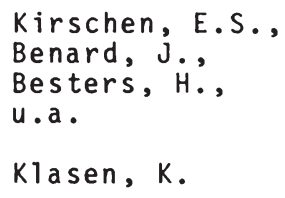

(1979) Zur Struktur politisch-ökonomischer Konjunkturzyklen, in: C.C. v. Weizsäcker (Hrsg.), Staat und Wirtschaft, Schriften des Vereins fur Socialpolitik N.F. 102, Berlin, $427-450$

(1979) Politisch ökonomische Modelle: Theoretische Ansätze und empirische Ergebnisse, in: Hillinger/Holler (1979), $86-125$

(1964) Economic Policy in Our Time. Band 1. 2nd Printing (1968). Amsterdam

(1970) Bonn entscheidet über den wirtschaftlichen Weg. Interview mit Bundesbankpräsident Karl Klasen. Der Volkswirt 24/20, $23-27$

(1971) Short-Term Fluctuations in U.S. Voting Behavior, 1896 - 1964. American Political Science Review 65, $131-143$

(1967) Keynes and the Keynesians. A Suggested Interpretation. American Economic Review, Papers and Proceedings 57, $401-425$

(1968) On Keynesian Economics and the Economics of Keynes - A Study in Monetary Policy. New York u.a.o.

(1974) Voting Behavior and Aggregate Policy Targets. Public Choice 18, Summer, $67-81$

(1948) The Burden of the National Debt, in: L.A. Metzler, u.a. (Hrsg.), Income, Employment and Public Policy, Essays in Honor of Alvin Hansen, New York, 255 - 276

(1978) Money Management and Institutions. Totowa

(1974) Einführung in die Währungspolitik. 8. Auflage. München

(1978) Zur Bedeutunq institutioneller Bedingungen für die Stabilitätspolitik - dargestellt am Beispiel GroBbritanniens. Zeitschrift für Wirtschafts- und Sozialwissenschaften $98,347-373$ 
Lucas, R.E. jr.

Luckett, D.G.

MacRae, C.D.

Mayer, $T$.

McCulloch, J.R.

MCMillin, W.D.

McMillin, W.D., Beard, T.R.

Meckling, W.H.

Meyer, L.H.

Miller, W.L., Mackie, M.

Modigliani, F.

Mosley, P.

Mueller, D.C.
(1975) An Equilibrium Model of the Business Cycle. Journal of Political Economy 83, $1113-1144$

(1976) Money and Banking. New York u.a.o.

(1977) A Political Model of the Business Cycle. Journal of Political Economy 85 , $239-263$

(1975) The Structure of Monetarism. Kredit und Kapital 8, 191 - 218 und 293 - 316

(1851) The Funding System; wiederabgedruckt in: J.R. McCulloch (1975), A Treatise on the Principles and Practical Influence of Taxation and the Funding System, edited with an introduction by D.P. O'Brien, Edinburgh London, $401-463$

(1981) A Dynamic Analysis of the Impact of Fiscal Policy on the Money Supply. Journal of Money, Credit and Banking 13,221-226

(1980) The Short-Run Impact of Fiscal Policy on the Money Supply. Southern Economic Journal $46,122-135$

(1978) Comments, in: Buchanan/Wagner $(1978 \mathrm{a}), 101-105$

(1980) Financing Constraints and the ShortRun Response to Fiscal Policy. Federal Reserve Bank of St. Louis Review 62, June/July, $24-31$

(1973) The Electoral Cycle and the Asymmetry of Government and Opposition Popularity: An Alternative Model Between Economic Conditions and Political Popularity. Political Studies $21,263-279$

(1964) Long-Run Implications of Alternative Fiscal Policies and the Burden of the National Debt, in: Ferguson (1964), 107-138

(1976) Towards a "Satisficing" Theory of Economic Policy. The Economic Journal 86 , $59-72$

(1976) Public Choice: A Survey. Journal of Economic Literature 14, $395-433$ 
Mueller, D.C.
Mueller, J.E.

Musgrave, R.A.

Musgrave, R.A.

Neumann, M.J.M.

Neumann, M.J.M.

Neumark, F.

Niskanen, W.A.

Nordhaus, W.D.

Nölling, W.

01 son, M.

$0 t t, A . E$.

Ott, A.E., Wagner, $A$.

Pierce, J.L.
(1979) Public Choice. London u.a.0.

(1970) Presidential Popularity from Truman to Johnson. The American Political Science Review $64,18-34$

(1958) Theorie der öffentlichen Schuld. Handbuch der Finanzwissenschaft. 2. Auflage. Band III. Tübingen, $68-137$

(1974) Finanztheorie. 2. Auflage. Studienausgabe. Tübingen

(1973) Zur relativen Bedeutung fiskalischer und monetärer Impulse. WSI-Mitteilungen 26 , $14-19$

(1981) Inflation und Staatsverschuldung, Zeitschrift für Wirtschafts- und Sozialwissenschaften $101,113-126$

(1949) Zur Problematik der Grenzen des Staatskredits. Zeitschrift für Nationalökonomie $12,513-5.37$

(1978) Deficits, Government Spending, and Inflation. What is the Evidence? Journal of Monetary Economics 4,591-602

(1975) The Political Business Cycle. The Review of Economic Studies 42, $169-190$

(1979) Staatsverschuldung ohne Ende? Kredit und Kapital 12, $472-503$

(1978) Comments, in: Buchanan/Wagner (1978a), $106-117$

(1963) Einführung in die dynamische wirtschaftstheorie. Göttingen

(1973) Materialien zu den Wachstumszyklen in der Bundesrepublik Deutschland, in:

A.E. Ott (Hrsg.), Wachstumszyklen. Ober eine neue Form der Konjunkturschwankungen, Schriften des Vereins für Socialpolitik N.F. 71, Berlin, $157-181$

(1978) The Myth of Congressional Supervision of Monetary Policy. Journal of Monetary Economics 4, $363-370$ 
Pissarides, C.A.

Pommerehne, W.W.

Pommerehne, W.W.

Pommerehne, W.W.

Pommerehne, W.W., Schneider, F.

Pommerehne, W.W., Schneider, F.

Pommerehne, W.W., Schneider, F.

Poole, W.

Potts, G.T.,

Luckett, D.

Poullain, L.

Puviani, A.
(1974) A Model of British Macroeconomic Policy 1955 - 1969. Manchester School of Economic and Social Studies 42

(1975) Offentliche Güter und öffentliche Ausgaben in der direkten Demokratie. Dissertation Konstanz

(1978) Institutional Approaches to Public Expenditure: Empirical Evidence from Swiss Municipalities. Journal of Public Economics $9,255-280$

(1981) Fiscal Illusion and Consumer Sovereignty: An Exploratory Study, in: B.M. Kent (Ed.), Advances in Consumer Research VIII, Washington

(1978) Fiscal Illusion, Political Institutions, and Local Public Spending. Kyklos 31 , $381-408$

(1980) Fiscal Illusion and Fiscal Policy in a Representative Democracy. Vortrag beim internationalen Symposium "Anatomy of Government Deficiencies", Diessen am Ammersee, Juli 1980

(1980a) Politico-Economic Interactions in Australia: Some Empirical Evidence. Economic Record $56,113-131$

(1979) The Monetary Deceleration: What Does it Mean and Why is it Happening? Brookings Papers on Economic Activity 1, $231-238$

(1978) Policy Objectives of the Federal Reserve System. The Quarterly Journal of Economics $92,525-534$

(1973) Für eine "demokratische" Autonomie Der Entscheidungsspielraum der Bundesbank -, in: D. Duwendag (Hrsg.), Macht und Ohnmacht der Bundesbank. Frankfurt/M., $36-52$

(1903) Teoria dell'Illusione Finanziara. Milano u.a.0.; in deutsch: Die Illusionen in der öffentichen Finanzwirtschaft (1960). Berlin. Die Seitenangaben beziehen sich auf die deutsche Quelle. 
Rattinger, $\mathrm{H}$.

Ricardo, D.

Robert, R.

Roberts, P.C.

Roesch, S.

Rose, $M$.

Rose, R.,

Peters, G.

Roth, D.

Rürup, B .

Sachverständigenrat zur Begutachtung der gesamtwirtschaftlichen Entwicklung

Sachverständigenrat zur Begutachtung der gesamtwirtschaftlichen Entwicklung

Scherer, $\mathrm{K}$.

Schlesinger, $H$.
(1980) Wirtschaftliche Konjunktur und politische Wahlen in der Bundesrepublik Deutschland. Ein Beitrag zur politikwissenschaftlich-statistischen Aggregatdatenanalyse. Berlin

(1923) Grundsätze der Volkswirtschaft und Besteuerung. 3. Auflage. Jena

(1978) Die Unabhängigkeit der Bundesbank. Analyse und Materialien. Kronberg

(1978) Idealism in Public Choice Theory. Journal of Monetary Economics 4,603-615

(1975) The Monetary-Fiscal Mix through Mid-1976. Federal Reserve Bank of St. Louis Review 57, August, $2-7$

(1980) Finanzwissenschaftliche Makrotheorie. München

(1978) Can Government Go Bankrupt? New York

(1973) Okonomische Variablen und Wahlverhalten. Politische Vierteljahresschrift 14 , $257-274$

(1980) Staatsverschuldung. Kein AnlaB zur Besorgnis. Wirtschaftsdienst $60,424-429$

(1967) Stabilität im Wachstum. Jahresgut achten 1967/68. Stuttgart - Mainz

(1975) Vor dem Aufschwung. Jahresgutachten 1975/76. Stuttgart - Mainz

(1977) Maßstäbe zur Beurteilung von konjunkturellen Wirkungen des öffentilichen Haushalts. Frankfurt/M. - Bern

(1975) Geldpolitik und Finanzpolitik. Zeitschrift für das gesamte Kreditwesen 28 , $1079-1082$ 
Schmah, U.

Schmölders, G.

Schmölders, G.

Schmölders, G.

Schneider, F.

Schwedes, H.

Searjant, G.

Shapiro, E.

Sharp, A.M.

Sharp, A.M.,

Flenniken, P.S.

Shoup, C.S.

Signer, A.

Simon, H.A.

Simpson, T.D.
(1967) Der Einkommensredistributionseffekt der Anleihefinanzierung bei unterschied1 ichen Zeitpräferenzen. Finanzarchiv N.F. $26,232-237$

(1959) Unmerkliche Steuern. Finanzarchiv N.F. $20,23-34$

(1970) Finanz- und Steuerpsychologie. Reinbek

(1975) Einführung in die Geld- und Finanzpsychologie. Darmstadt

(1979) Ein politisch-ökonomisches Modell des Zentralbankverhaltens be $j$ endogenem Staat, in: C.C. v. Weizsäcker (Hrsg.), Staat und Wirtschaft, Schriften des Vereins für Socialpolitik N.F. 102, Berlin, 473 497

(1972) Der Bund am Kapitalmarkt. Vorschläge zur staatlichen Anleihepolitik. Berlin

(1976) People's Banker - or Chancellor's Pet? The Sunday Times vom 17.12.1976, 60 61

(1975) Understanding Money. New York u.a.0.

(1979) On Budget Deficits as a Major Cause of Inflation: A Reply. Public Finance Quarterly $7,387-390$

(1978) Budget Deficits. A Major Cause of Inflation? Public Finance Quarterly 6, 115 127

(1962) Debt Financing and Future Generations. The Economic Journal 72, 887 - 898

(1980) Budgetgleichgewicht und Erhaltung der effektiven Nachfrage: Ein Zielkonflikt? Bern u.a.o.

(1955) A Behavioral Model of Rational Choice. Quarterly Journal of Economics 69/1,99- 118

(1976) Money, Banking, and Economic Analysis. Englewood Cliffs 
Smith, W.L.,
Teigen, R.L.
Spann, R.M.

v. Spindler, J., Becker, W.,

Starke, E.

Spindler, Z.A.

Stigler, G.J.

Stimson, J.A.

Stutze 1, W.

Teschner, M.

Theil, H.

Tobin, J.

Tobin, J.

Tobin, J.
(1974) Readings in Money, National Income, and Stabilization Policy. Homewood, Ill.

(1977) Rates of Productivity Change and the Growth of State and Local Governmental Expenditures, in: Borcherding (1977), 100 129

(1973) Die Deutsche Bundesbank. 4. Auflage. Stuttgart u.a.0.

(1979) On Budget Deficits as a Major Cause of Inflation: A Comment. Public Finance Quarterly 7,381-386

(1973) General Economic Conditions and $\mathrm{Na}-$ tional Elections. American Economic Review, Papers and Proceedings 63, $160-167$

(1976) Public Support for American Presidents: A Cyclical Model. Public Opinion Quarterly $40,1-21$

(1978) Ober- und Untergrenzen der öffentlichen Verschuldung. Kredit und Kapital 11 , $429-450$

(1980) Hält die Crowding-out-These kreislauftheoretischer Kritik stand? Deutsches Institut für Wirtschaftsforschung. Vierteljahreshefte zur Wirtschaftsforschung 47 , $121-125$

(1964) Optimal Decision Rules for Government and Industry. Amsterdam u.a.0., 2nd Printing 1968

(1961) Money, Capital and Other Stores of Value. American Economic Review, Papers and Proceedings $51,26-37$

(1963) An Essay on the Principles of Debt Management, in: Fiscal and Debt Management Policies, 143 - 218; in deutsch erschienen als: Grundsätze der Geld-und Staatsschuldenpolitik (1978), Baden-Baden

(1965) The Burden of the Public Debt: A Review Article. The Journal of Finance 20 , $679-682$ 
Tobin, J.

Tolkemitt, G.

Trapp, P.

Tufte, E.T.

Tullock, G.

Tullock, G.

Ulmer, M.J.

Vickrey, W.

Vining, D.R. jr., Elwertowski, T.C.

Wagner, A.

Wagner, R.E.

Wagner, R.E.

Wagner, R.E.

Wagner, R.E.

Wagstaff, J.V.
(1978) Comment from an Academic Scribbler. Journal of Monetary Economics 4, $617-625$

(1975) Zur Theorie der langfristigen Wirkungen öffentlicher Verschuldung. Tübingen

(1976) Geldmenge, Ausgaben und Preisanstieg in der Bundesrepublik Deutschland. Tübingen

(1975) Determinations of the outcome of Midterm Congressional Elections. The American Political Science Review 69,812 - 826

(1971) Public Decisions as Public Goods. Journal of Political Economy 79,913 - 918

(1978) Comments, in: Buchanan/Wagner (1978a), $70-74$

(1978) Comments, in: Buchanan/Wagner (1978a), $74-77$

(1961) The Burden of the Public Debt: Comment. American Economic Review 51, 132 - 137

(1976) The Relationship Between Relative Prices and the General Price Level. American Economic Review $66,699-708$

(1892) Grundlegung der politischen Okonomie. 1. Teil. 1. Halbband. 3. Auflage. Leipzig

(1976) Revenue Structure, Fiscal Illusion, and Budgetary Choice. Public Choice 25, Spring, $45-61$

(1977) Economic Manipulation for Political Profit: Macroeconomic Consequences and Constitutional Implications. Kyklos 30,395 409

(1977a) Revenue Structure, Fiscal Illusion, and Budgetary Choice: Reply. Public Choice $29,131-132$

(1980) Boom and Bust: The Political Economy of Economic Disorder. Journal of Libertarian Studies 4 , Winter, $1-37$

(1965) Income Tax Consciousness under Withholding. Southern Economic Journal 32, 73 80 
Walters, A.A.

We intraub, R.E.

We ise, H.

West, E.G., Corke, G.

West, E.G., Winer, S.L.

Whittlesey, C.R.

Whittlesey, C.R., Freedman, A.M., Herman, E.S.

Wildavsky, A.

Willms, M.

Willms, M.

Wissenschaftlicher Beirat beim Bundesministerium der Finanzen

Wissenschaftlicher Beirat beim Bundesministerium der Finanzen
(1967) How to Make a Benefit of the Burden of National Debt. National Tax Journal 20 , $316-318$

(1978) Congressional Supervision of Monetary Policy. Journal of Monetary Economics $4,341-362$

(1963) Aktuelle Probleme der Geld-und Kreditpolitik aus amerikanischer Sicht. Zum Bericht der Commission on Money and Credit. Weltwirtschaftliches Archiv 90/T, 148-187

(1980) Tax Constraints on Leviathan. Some Second Thoughts on the Constitutional Choice Calculus. Journal of Public Economics 13 , $395-401$

(1980) Optimal Fiscal Illusion and the Size of Government. Public Choice 35,607-622

(1963) Power and Influence in the Federal Reserve System. Economica $30,33-44$

(1964) Money and Banking. Analysis and Poi icy. New York

(1980) How to Limit Government Spending. Berkeley u.a.0.

(1968) Der Einsatz der Staatsschuld als geldpolitisches Instrument. Kredit und $\mathrm{Ka}-$ pital 1, 405-438

(1978) Volkswirtschaftliche Wirkungen einer zunehmenden Staatsverschuldung. Wirtschaftsdienst $58,439-445$

(1975) Gutachten zur Lage und Entwicklung der Staatsfinanzen in der Bundesrepublik Deutschland. Bonn

(1979) Gutachten zur Schuldenstrukturpolitik des Staates. Schriftenreihe des Bundesministeriums der Finanzen 27. Bonn 
Wissenschaft 1 icher Beirat beim Bundesministerium der

Finanzen

Wissenschaftlicher Beirat beim Bundes ministerium für Wirtschaft und Finanzen

Wood, J.H.

Yohe, W.P.

Zecher, $R$.

Ziffzer, S.

Ziffzer, S.
(1980) Gutachten zum Begriff der öffentlichen Investitionen - Abgrenzungen und Folgerungen im Hinblick auf Artikel 115 Grundgesetz. Schriftenreihe des Bundesministeriums der Finanzen 29. Bonn

(1972) Gutachten zur Finanzierung eines höheren Staatsanteils am Sozialprodukt, in: Bundesministerium der Finanzen (Hrsg.), Der Wissenschaftliche Beirat beim Bundesministerium der Finanzen. EntschlieBungen, Stellungnahmen und Gutachten 1949 - 1973 (1974), Tübingen

(1967) A Model of Federal Reserve Behavior, in: G. Horwich, R.D. Irwin (Eds.), Monetary Process and Poi icy: A Symposium, Homewood, I11., $135-166$

(1968) The Open Market Committee Decision Process, in: A.D. Entine (Ed.), Monetary Economics: Readings, Belmont, $134-148$

(1971) Money and Congress. A Review of Congressional Activity Relating to Monetary Policy. Journal of Money, Credit and Banking 3, $680-692$

(1980) Grenzen der Staatsverschuldung. Zeitschrift für das gesamte Kreditwesen 33, 500 $-505$

(1980a) Okonomische Grenzen der staatlichen Kreditaufnahme. Berlin 


\section{HOHENHEIMER VOLKSWIRTSCHAFTLICHE SCHRIFTEN}

Band 1 Walter Deffaa: Anonymisierte Befragungen mit zufallsverschlüsselten Antworten. Die Randomized-Response-Technik (RRT). Methodische Grundlagen, Modelle und Anwendungen. 1982.

Band 2 Thomas Michael Baum: Staatsverschuldung und Stabilisierungspolitik in der Demokratie. Zur neoinstitutionalistischen Kritik der keynesianischen Fiskalpolitik. 1982. 
Folkers, Cay

VERMÖGENSVERTEILUNG UND STAATLICHE AKTIVITÄT

Zur Theorie distributiver Prozesse im Interventionsstaat

Frankfurt/M., Bern, 1981. VIII, 380 S.

Finanzwissenschaftliche Schriften. Bd. 14

ISBN 3-8204-6191-4

br. sFr. 49.-

Die Untersuchung hat das Ziel, eine theoretische Analyse der Funktionen und Bestimmungsgründe der Vermögensverteilung unter besonderer Berücksichtigung der Beziehungen zu den ökonomischen Aktivitäten des Staates zu entwerfen. Sie will zu einer Neuformulierung von Konzepten und Systemzusammenhängen der Vermögensverteilung beitragen, um eine fundierte Beurteilung verteilungspolitischer Massnahmen des Staates bezüglich des Vermögens zu ermöglichen.

Aus dem Inhalt: U.a. Die Vermögensfunktionen - Die langfristige Inzidenz vermögenspolitischer Massnahmen - Die private Vermögensverteilung aus neoklassischer und neokeynesianischer Sicht - Machtfunktion und Vermögensverteilung - Die Vermögensverteilung zwischen ökonomischen Abhängigkeiten und staatlichem Eingriff - Private Vermögensverteilung und öffentliches Vermögen.

Ott, Günter

\section{EINKOMMENSUMVERTEILUNGEN IN DER GESETZLICHEN KRANKENVERSICHERUNG}

Eine quantitative Analyse

Frankfurt/M., Bern, 1981..254 S.

Finanzwissenschaftliche Schriften. Bd. 16

ISBN 3-8204-6959-1

br. sFr. 57.-

In der gesetzlichen Krankenversicherung (GKV) bestehen so vielfältige Umverteilungswirkungen wie sonst bei keinem anderen System unserer sozialen Sicherung. Ihre Grössenordnungen sind allerdings weitgehend unbekannt. Diese zu quantifizieren ist das Hauptziel der Analyse. Darüber hinaus wird untersucht, inwieweit die GKV von ihrer verteilungspolitischen Konzeption her überhaupt in der Lage ist, konsistente Umverteilungsprozesse zu vollziehen. Hiervon ausgehend werden abschliessend Möglichkeiten der Reform geprüft.

Aus dem Inhalt: Analyse zeitpunktbezogener Umverteilungswirkungen - Grössenordnungen lebenszeitbezogener Umverteilungen - Analyse der verteilungspolitischen Konzeption der gesetzlichen Krankenversicherung - Möglichkeiten der Reform.

\section{Verlag Peter Lang $\cdot$ Bern und Frankfurt am Main}

Auslieferung: Verlag Peter Lang AG, Jupiterstr. $15, \mathrm{CH}-3000$ Bern 15

Telefon $(0041 / 31) 321122$. Telex verl ch 32420

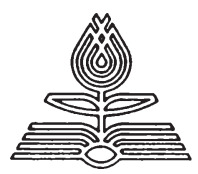

